\title{
Measurement of the inclusive jet cross section with the ATLAS detector at the Large Hadron Collider
}

\author{
Caterina Doglioni \\ University of Oxford \\ Merton College
}

Thesis submitted in partial fulfilment of the requirements for the degree of Doctor of Philosophy November 24, 2011 


\section{Abstract}

This thesis presents the measurement of the inclusive jet cross section using data collected in 2010 by the ATLAS detector, with a particular focus on the reconstruction and calibration techniques used for jets in this measurement and on the estimate of the systematic uncertainty on their energy scale. The inclusive jet cross section measurement is used as input to fits of parton distribution functions. Although the PDF analysis in this thesis is preliminary and its main purpose is to serve as a proof of principle for future studies, improvements in the knowledge of the gluon density are observed thanks to the inclusion of ATLAS data. 


\section{Acknowledgements}

There are many people to whom I am indebted. First of these is my supervisor, Amanda CooperSarkar. She has been incredibly supportive when I have needed it, and at the same time has given me the freedom to pursue work of my own choosing. Much of the work in this thesis was influenced by Tancredi Carli, whose encouragement, guidance and approachability during my time at CERN made much of this thesis write itself.

Thanks to Cigdem Issever for always helping me see the big picture, to the other Oxford group members (Tony Weidberg, Hugo Beauchemin and Todd Huffmann in particular) for the advice on both physics and the practical side of things and to Alan Barr for being the perfect College Advisor. I am grateful to the Oxford Particle Physics secretaries for turning bureaucracy into something easy that could be handled with a smile. Thanks also to Peter Loch, for giving me chances to take over both the JetPerformance code and his kitchen, even though he's a master of both.

My D. Phil. course in Oxford was supported by the concrete help of a number of Foundations: the Rotary Club (Clubs of Feltre (BL) and Pinner, with special thanks to Ian and Judy Shave for always being more than wonderful counsellors), the Blanceflor, Scatcherd and Charterhouse Foundations, the Stapley Trust, the British Foundation of Women Graduates and the Fondazione Della Riccia.

Thanks to Dag, Toshi, Michael and the other JetEtMiss activists for making me feel part of a strong team where deadlines were challenging but still fun. In particular, thanks to Pier-Olivier: without suggestions (and live office-playing) of an excellent soundtrack, deriving a JES uncertainty wouldn't have been so epic and HAppy. Dag (again), Paolo, Bogdan, Pavel, Shima, Mario, Pavel, Eric and all the other inclusive jet/dijet cross section analysers at CERN deserve special thanks for answering all my questions at all times and for not complaining about the burned cakes I sometimes brought to meetings. Thanks to Frederik and Yumi for teaching me things about jets, (recursive) limit setting and proper screeching, and to Heidrun for the patience whenever she had to listen to all of these. I am also indebted to Sing and Teresa for overseas delivery of ramen, Canadian coke and advice when needed.

Thanks to the Oxford Physics peeps: Sam+James=JAM, Dan, Alex, Phil, theBODDY and the younglings for filling the days in the department with banter. I would also like to thank Steven, Mike, Francoise, Mel, Robin, the ducks that sift primes in the Cherwell and the lesser waterchickens since they all contributed to make Oxford sunny regardless of British weather. Thanks to the LTA for the tablesnakes, coffees, ski trips and nights out. Special thanks to the LTA girls for keeping up the tradition of girly nights. Thanks to the colleghe, Market, Chiara+Gae, the Toni, the Valery, Laura, Nadia, Olivia and Dirk for all the skype/email/live support.

Thanks to my family for supporting me all along this path, even if this meant I'd be mostly straying far from home (and would often be quite busy when at home), and for always being willing to listen to stories on the getti adronici. Finally thanks to Conor, for being there to share with me the enthusiasm and curiosity for this subject, unconditionally, at all times. Starting from the first smiles when looking up from opposite laptops, it is the passion for our work that brings us to share a lifetime of adventures - this is why this thesis is dedicated to him. 


\section{Declaration}

This dissertation is the result of my own work, except where explicit reference is made to the work of others, and has not been submitted for another qualification to this or any other university. This dissertation does not exceed the page limit for the respective Degree Committee.

Caterina Doglioni

\section{Personal contributions}

Due to the large nature of particle physics experimental collaborations, the work in this thesis relies on the results of a large number of individual researchers and analysis subgroups. Leading contributions by the author presented in this thesis are outlined below, subdivided by thesis chapter.

Chapter 4: Jet reconstruction performance The author has performed jet energy resolution studies for low $p_{T}$ jets and validation of calibration constants in Monte Carlo before central processing of data for various software releases. These studies have been performed using the JETPERFORMANCE software, written and deployed over the course of the first year of this D. Phil. program.

Chapter 5: Jet Energy Scale Uncertainty The author has been the main author of the Monte-Carlo based analysis for the estimate of the jet energy scale uncertainty, and has combined the final results and delivered them to the collaboration using a centralised software tool.

Chapter 6: Inclusive Jet Cross Section The author has performed cross-checks of Monte Carlo and data spectrum (for selected data runs), validated the final data and theory spectra and uncertainty values, and partecipated actively to the discussion on the correlation of uncertainties to be treated as nuisance parameters.

Chapter 7: Impact of ATLAS jet data in PDF fits The author has adapted the ZEUS PDF fitting code to include ATLAS data, and has performed a PDF analysis for the evaluation of the impact of ATLAS jet data on the PDF. Cross-checks with the fitting code from the H1 experiment have also been performed. 


\section{Contents}

$\begin{array}{ll}\text { Introduction } & 1\end{array}$

1. Theory framework: QCD $\mathbf{5}$

1.1. Introduction to the Standard Model . . . . . . . . . . . . . . . . 5

1.2. Key concepts in Quantum Chromodynamics . . . . . . . . . . . . . . 6

1.2.1. Asymptotic freedom and confinement . . . . . . . . . . . . 7

1.2.2. Renormalisation, scale violations and parton distribution functions . 10

1.2.3. QCD Factorisation . . . . . . . . . . . . . . . . 13

1.3. Infrared safety in inclusive processes: jets . . . . . . . . . . . . . . . . . . 14

1.3.1. Inclusive jet production . . . . . . . . . . . . . . 15

1.3.2. Motivations to measure the inclusive jet cross section . . . . . . . . 16

1.4. Theoretical tools . . . . . . . . . . . . . . . . . . 16

1.4.1. NLO calculation software and convolution with PDFs . . . . . . . 16

1.4.2. Parton shower Monte Carlo generators . . . . . . . . . . . . . 17

2. The ATLAS Experiment 21

2.1. CERN and the Large Hadron Collider . . . . . . . . . . . . . . . . . . . . . 21

2.1.1. Running conditions of the LHC accelerator . . . . . . . . . . . . 22

2.2. Overview of the ATLAS detector and coordinate system . . . . . . . . . 23

2.3. The ATLAS inner detector system . . . . . . . . . . . . . . . 25

2.4. The ATLAS muon system . . . . . . . . . . . . . . . . 26

2.5. The ATLAS calorimeter system . . . . . . . . . . . . . 26

2.5.1. Electromagnetic calorimeters . . . . . . . . . . . . . . 27

2.5.2. Hadronic calorimeters . . . . . . . . . . . . . . . . . . 32

2.6. Simulation of particle interactions in the ATLAS detector . . . . . . . . . 34

2.7. Software, computing and data analysis . . . . . . . . . . . . 35

2.8. Data used in this thesis . . . . . . . . . . . . . . . . 35

2.9. Trigger system . . . . . . . . . . . . . . . . . . . 35

3. Jet reconstruction in ATLAS 39

3.1. Jet algorithms and inputs to jet reconstruction . . . . . . . . . . . . . . . 39

3.1.1. Theoretical requirements for a jet algorithm . . . . . . . . . . . . . 40

3.1.2. Jet definition in ATLAS . . . . . . . . . . . . . . . . . . . . 41

3.1.3. Inputs to jet reconstruction . . . . . . . . . . . . . . . . . . 42

3.2. Jet energy scale calibration in ATLAS . . . . . . . . . . . . . . . . 44

3.2.1. EM+JES calibration scheme . . . . . . . . . . . . . . 46

3.2.2. Other calibration schemes . . . . . . . . . . . . . . . . 49

4. Jet reconstruction performance 55

4.1. Jet energy resolution . . . . . . . . . . . . . . . . . . . 56

4.1.1. In-situ determination of the jet energy resolution . . . . . . . . . . 56 
4.1.2. Results from in-situ methods and comparison to Monte Carlo . . . . 57

4.2. In-situ validation of the jet energy scale calibration . . . . . . . . . . . . . . 59

4.2.1. Dijet balance . . . . . . . . . . . . . . . . . . . 60

4.3. Monte Carlo based performance studies . . . . . . . . . . . . . . . . . . 63

4.3.1. Energy and $p_{T}$ response . . . . . . . . . . . . . . . . . . . . 64

4.3.2. Effect of pile-up on the jet response . . . . . . . . . . . . . . . . . 64

4.3.3. Jet energy resolution from Monte Carlo . . . . . . . . . . . . . . . . 67

4.3.4. Monte Carlo purity and efficiency . . . . . . . . . . . . . . . . 68

5. Jet Energy Scale Uncertainty $\quad 71$

5.1. Analysis details . . . . . . . . . . . . . . . . . . . . 72

5.1.1. Selection of jets for systematic studies in Monte Carlo studies . . . . 73

5.2. Sources of jet energy scale uncertainty . . . . . . . . . . . . . . 74

5.2.1. Uncertainty due to the JES calibration . . . . . . . . . . . 74

5.2.2. Uncertainty on the calorimeter response . . . . . . . . . . . 75

5.2.3. Uncertainties due to the detector simulation . . . . . . . . . . 80

5.2.4. Uncertainties due to the event modelling in the Monte Carlo generators 86

5.2.5. Uncertainties due to the relative calibration (endcap/forward regions) 87

5.3. Uncertainty due to multiple interactions . . . . . . . . . . . . . . . . . 89

5.4. Combination of JES uncertainty sources . . . . . . . . . . . . . . 91

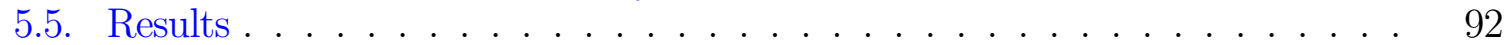

5.5.1. Validation of the JES uncertainty with in-situ techniques . . . . . 95

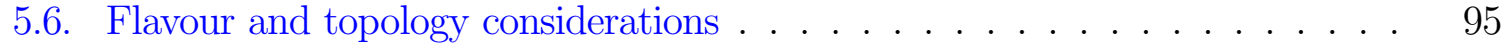

5.7. JES uncertainty correlations . . . . . . . . . . . . . . . . 97

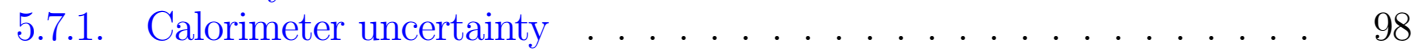

6. Inclusive Jet Cross Section 103

6.1. Analysis overview and datasets used . . . . . . . . . . . . . . . 104

6.2. Event and jet selection . . . . . . . . . . . . . . . . . 105

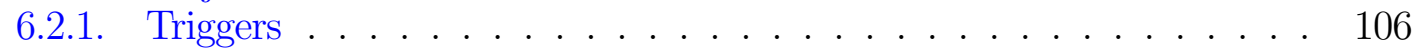

6.2.2. Jet identification . . . . . . . . . . . . . . . . . . . . 109

6.2.3. Stability cross checks and effect of pile-up . . . . . . . . . . . . . 111

6.3. Data corrections for detector effects (unfolding) . . . . . . . . . . . . 111

6.3.1. Iterative Dynamically Stabilized (IDS) unfolding . . . . . . . . . . 114

6.4. Systematic uncertainties . . . . . . . . . . . . . . . . 116

6.4.1. Jet energy scale uncertainty . . . . . . . . . . . . . . 116

6.4.2. Jet energy resolution uncertainty _. . . . . . . . . . . . . 117

6.4.3. Jet identification and trigger . . . . . . . . . . . . . . . 118

6.4.4. Jet angular resolution uncertainty . . . . . . . . . . . . . . . 118

6.4.5. Reconstruction efficiency . . . . . . . . . . . . . . . . . . . . . 119

6.4.6. Unfolding . . . . . . . . . . . . . . . . . . . . . . . . . 119

6.4.7. Luminosity measurement . . . . . . . . . . . . . . . . . . 120

6.4.8. Overall systematic uncertainties and correlations . . . . . . . . . 120

6.5. Theoretical calculations for comparison to data . . . . . . . . . . . . . . 124

6.5.1. Theoretical uncertainties and uncertainties on non perturbative corrections 125

6.6. Experimental Results and comparison to theory . . . . . . . . . . . . 127

7. Impact of ATLAS jet data on PDF fits 135

7.1. Extraction of parton distribution functions . . . . . . . . . . . 137

7.1.1. PDF fits . . . . . . . . . . . . . . . . . . 138 
7.2. Treatment of uncertainties . . . . . . . . . . . . . . . . . . 138

7.2.1. Treatment of correlated experimental errors in PDF fitting . . . . . 139

7.3. Details on PDF analysis with ATLAS and HERA data . . . . . . . . . . . 142

7.3.1. HERAPDF dataset . . . . . . . . . . . . . . . . . . . . . . 142

7.3.2. Starting scale parametrisation and parameter fitted . . . . . . . . . 142

7.3.3. HERAPDF: model and parametrisation uncertainties . . . . . . . . 145

7.4. Results: HERAPDF1.5 and ATLAS data . . . . . . . . . . . . . . . . . . . 145

7.4.1. Impact of ATLAS jet data on PDF fits . . . . . . . . . . . . . . 146

7.4.2. Determination of $\alpha_{s}\left(M_{Z}^{2}\right)$ with ATLAS jet data . . . . . . . . . . . 151

7.4.3. Impact of reduced JES uncertainty . . . . . . . . . . . . . . . . 153

7.4.4. Conclusions on the PDF analysis . . . . . . . . . . . . . . . . 154

$\begin{array}{ll}\text { Conclusions and outlook } & 154\end{array}$

$\begin{array}{lr}\text { Bibliography } & 159\end{array}$

A. In-situ validation of the jet energy scale and resolution $\quad 171$

A.1. Estimate of the jet energy resolution with data . . . . . . . . . . . . . 171

A.2. In-situ validation of the jet energy scale . . . . . . . . . . . . . . . . 174

A.2.1. Photon-jet balance . . . . . . . . . . . . . . . . . . 174

A.2.2. Balance between a high- $p_{T}$ jet recoiling against lower- $p_{T}$ jets $\ldots . . .175$

A.2.3. Comparison of calorimeter to track jet transverse momentum . . . . 176

$\begin{array}{ll}\text { B. Jet performance analysis tools } & 179\end{array}$

B.1. The JetFrameWORKToOLS package . . . . . . . . . . . . . . . . . . . . 179

B.2. The JetPerformance package . . . . . . . . . . . . . . . . . . . . 180

B.2.1. Jet Performance workflow . . . . . . . . . . . . . . . . . . 180

B.3. Use cases . . . . . . . . . . . . . . . . . . . . . . . . . . 181

B.3.1. Use case: validation of the jet energy scale calibrations . . . . . . . . . 181

B.3.2. Use case: jet energy scale uncertainty in ATLAS . . . . . . . . . . . 182

$\begin{array}{ll}\text { C. JES uncertainty plots } & 183\end{array}$

D. First estimate of the JES uncertainty (June 2010) 187

D.1. Analysis details . . . . . . . . . . . . . . . . . . . . 187

D.1.1. Jet calibration for the first $17 \mathrm{nb}^{-1}$ of ATLAS data . . . . . . . . . 187

D.1.2. Selection of jets for systematic studies in Monte Carlo studies . . . . 188

D.2. Sources of jet energy scale uncertainty . . . . . . . . . . . . . . . . . 188

D.2.1. Uncertainty due to the JES calibration . . . . . . . . . . . . . 188

D.2.2. Calorimeter response and dead material uncertainty . . . . . . . . . 190

D.2.3. Uncertainties due to the detector simulation . . . . . . . . . . . . 193

D.2.4. Uncertainties due to the event modelling in the Monte Carlo generators 195

D.2.5. Endcap uncertainty due to relative calibration . . . . . . . . . 196

D.3. Uncertainty due to multiple interactions . . . . . . . . . . . . . . . 196

D.4. Combination of JES uncertainty sources . . . . . . . . . . . . . . . . . 199

D.5. Results . . . . . . . . . . . . . . . . . . . 200

E. Tables for the inclusive jet cross section measurement 203 
"Uncertain ways unsafest are" - John Denham 


\section{Introduction}

The first months of proton-proton collisions at a centre of mass energy of $7 \mathrm{TeV}$ delivered by the Large Hadron Collider and recorded by the ATLAS experiment have provided data to probe quantum chromodynamics (QCD) at scales never reached before. While precision tests of strong interactions are interesting in their own right, QCD also provides one of the main backgrounds to many New Physics measurements; furthermore, it is also through tests of QCD that New Physics may be discovered. Hadronic jets are a fundamental ingredient for precision tests of QCD: understanding and measuring their performance starting from the very first data is crucial in the LHC environment. This thesis presents the measurement of the inclusive jet cross section using data collected in 2010 by the ATLAS detector, with a particular focus on the reconstruction and calibration techniques used for jets in this measurement and on the estimate of the systematic uncertainty on their energy scale. PDF fits have been performed comparing the inclusive jet cross section data to theory. Although the PDF analysis performed within this thesis is preliminary and its main purpose is to serve as a proof of principle for future studies, improvements in the knowledge of the gluon density are observed thanks to the inclusion of ATLAS data.

After a brief introduction on the theoretical framework of the Standard Model of Particle Physics and QCD in Chapter 1, the ATLAS detector is described in Chapter 2. Chapters 3 and 4 are devoted to understanding the treatment of jets and measuring the performance of their reconstruction in the Monte Carlo simulation and in data. A correct estimate of the energy of jets (jet energy scale) is input to many physics analyses, and the uncertainty on this estimate is the dominant experimental systematic for many QCD measurements and for the inclusive jet cross section measurement. The estimate of the jet energy scale uncertainty for the first ATLAS data is described in Chapter 5. As detailed in Chapter 7, the inclusive jet cross section measurement described in Chapter 6 is used as input to fits of parton distribution functions to improve the knowledge of the gluon PDF and estimate the value of the strong coupling constant. 


\section{Chapter 1.}

\section{Theory framework: QCD}

The theoretical framework of particle physics is called the Standard Model (SM). The SM is a $\mathrm{SU}(3) \otimes \mathrm{SU}(2) \otimes \mathrm{U}(1)$ gauge theory, describing the strong (QCD), weak and electromagnetic interactions respectively. It describes the interactions of the fundamental components of matter, quarks, leptons and gauge bosons, according to three of the four forces in nature. Measurements of the Standard Model parameters have confirmed the theoretical predictions to increasing precision, but evidence that new physics phenomena are needed to extend the knowledge of the fundamental components of matter could be found at the LHC.

This chapter outlines the theoretical framework that underlies the measurement described in this thesis, and the tools used to simulate hadron interactions for the theoretical predictions used for comparison with the measurement. An overview of the Standard Model in terms of the electroweak interactions is given in Section 1.1. A brief description of the Quantum Chromodynamics concepts used in this thesis follows in Section 1.2. Section 1.3 introduces the concept of jets as tools to obtain predictable and measurable quantities and the motivations to measure the inclusive jet cross section. Finally Section 1.4 outlines the theoretical and computational tools used for the simulation of hadronic interactions.

\subsection{Introduction to the Standard Model}

The Standard Model is the theoretical framework that describes strong, weak and electromagnetic interactions between fundamental particles (see $[1,2]$ for a concise introduction and further references). It is a non-abelian gauge theory: the transformations of the symmetry group do not commute in the case of the QCD and weak groups. Gauge invariance, defined as the invariance of the theory under local transformations, is a fundamental property of the theory. Gauge invariance defines the particle content of the Standard Model (as shown in Figure 1.1), as the 


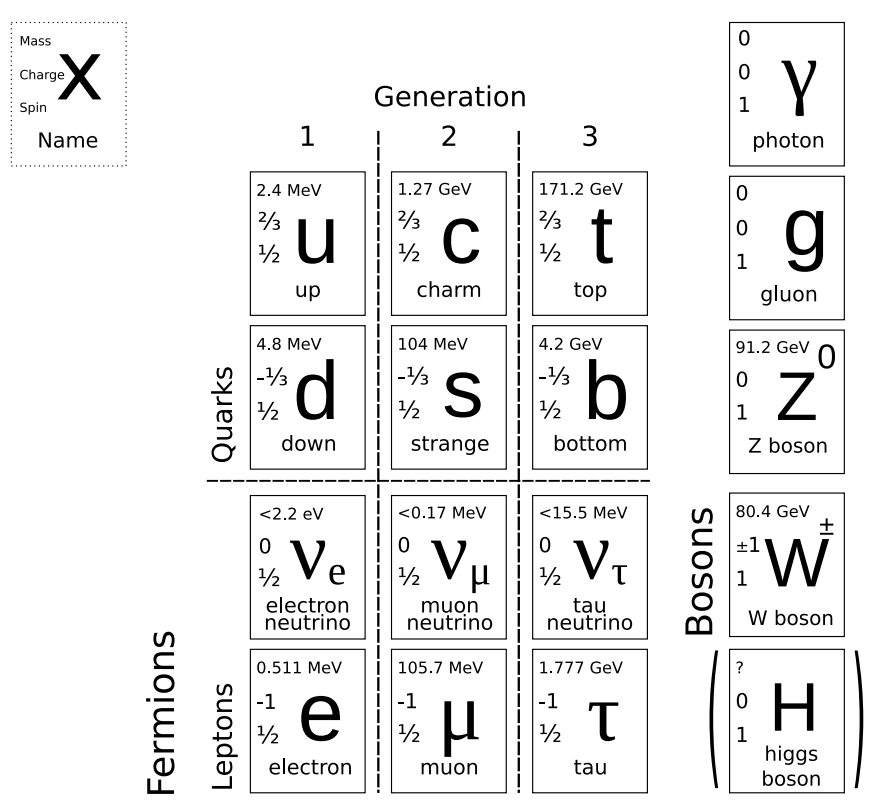

Figure 1.1.: The fundamental particles of the Standard model, sorted according to family, generation and mass. Figure from [5].

quanta of the gauge invariant fields are identified with the five gauge bosons. These are the photon, the gluon, the $\mathrm{W}^{+/-}$and $\mathrm{Z}$ and they act as mediators of the forces. A consequence of the non-abelian character of the theory is that the gauge bosons for the QCD and weak forces enjoy self-interactions. In addition to the gauge bosons, the Standard Model is composed of 12 fermions of spin $1 / 2$, grouped in three lepton and three quark families. These are considered point-like, as there is no evidence of any internal structure of leptons or quarks to date. The mass of the Standard Model particles can be introduced using the Higgs mechanism [3, 4] through spontaneous symmetry breaking that leads to the appearance of a new boson. The Higgs boson has not yet been observed, and its discovery is sought by the general purpose experiments at the Large Hadron Collider.

\subsection{Key concepts in Quantum Chromodynamics}

Quantum chromodynamics is the theory of strong interactions. Its fundamental actors are quarks and gluons, which are confined in the nucleon but act as free at sufficiently small scales (and high energies). The latter behaviour is called asymptotic freedom. The direct consequence of confinement is that free quarks and gluons are never observed experimentally, and their final state is a collimated shower of hadrons.

The development of QCD was posterior to that of QED: while the latter was highly successful in the mid-Sixties, no information about the components of the nucleons was available, and 
strong interactions were commonly described using general principles and the exchange of mesons [6] although the basis for theories that could eventually accommodate QCD had also been developed [7]. A framework called Eightfold Way [8] had been developed to organize subatomic baryons and mesons into octets. Its connection to an underlying pointlike structure of hadrons came after the so-called heroic age of deep inelastic scattering measurements interpreted using the parton model [9]. These experiments and subsequent interpretations showed that the probes scattered against pointlike, spin 1/2 constituents of the nucleons that are the quarks (some relevant papers are collected in Reference [10]). The presence of spin 1 gluons was also inferred using kinematic considerations in terms of the total momentum shared by the quarks. The QCD equivalent of the electromagnetic charge for QCD is the colour charge: (anti)quarks can take three (anti)colours (red, green and blue and their counterparts) while the eight interacting gluons exist in a superposition of colour and anticolour states.

SU(3) QCD was established as a theoretical framework for strong interactions only following the discovery of asymptotic freedom as a consequence of the theory's renormalisability [11]. These concepts will be explored further in the following section. Only a brief overview of the concepts and formulas directly used in this thesis is given, while more material is available in the references quoted.

\subsubsection{Asymptotic freedom and confinement}

Gluons in QCD are massless, therefore the theory contains divergences that manifest themselves as infinite results in theoretical cross section calculations. A renormalisation procedure is necessary in order to allow the theory to give meaningful (non infinite) results that can be compared to the experimental measurements. This is achieved by effectively subtracting these infinities through counterterms embedded in so-called bare parameters that are not measurable. The renormalisation procedure introduces a correction to the 'renormalised parameter' depending on the renormalisation scale $\mu_{R}$ (that can be interpreted as the scale at which the subtraction is made) and on the physical scale at which the measurement is made, taken as the squared momentum transfer $Q^{2}$ in the following. Imposing the independence of the final result (all orders of perturbation theory) from the renormalisation scale allows one to derive an explicit form for the renormalised parameter ${ }^{1}$. As an example of a renormalised parameter, the strong coupling constant is shown in Equation 1.1:

\footnotetext{
${ }^{1}$ The dependence on $\mu_{R}$ is still present when truncating the theoretical calculation at a given order: this introduces an uncertainty on the theory predictions.
} 


$$
\alpha_{s}\left(Q^{2}\right)=\frac{\alpha_{s}\left(\mu_{R}^{2}\right)}{1+\alpha_{s}\left(\mu_{R}^{2}\right) \beta_{0} \ln \left(Q^{2} / \mu_{R}^{2}\right)} .
$$

The coupling constant, initially scale-invariant, becomes a function of the scale of the process: the common terminology used is that the coupling constant runs with energy. The theoretical and experimental results for the running coupling constant $\alpha_{s}$ as of 2009 are shown in Figure 1.2. Contrary to QED, where the coupling constant increases with the scale of the process $\left(Q^{2}\right.$ in Figure 1.2), gluon self interactions lead the $\beta_{0}$ function to be negative. The coupling constant therefore is sizeable at low $Q^{2}$ (leading to confined partons) and decreases as a function of $Q^{2}$ (leading to asymptotic freedom).

Asymptotic freedom and confinement are therefore deeply linked to the gauge structure of QCD that leads to gluon self interactions. This was demonstrated by Gross and Wilczek in 1973 [12,13], and recognized with a Nobel Prize in 2004.

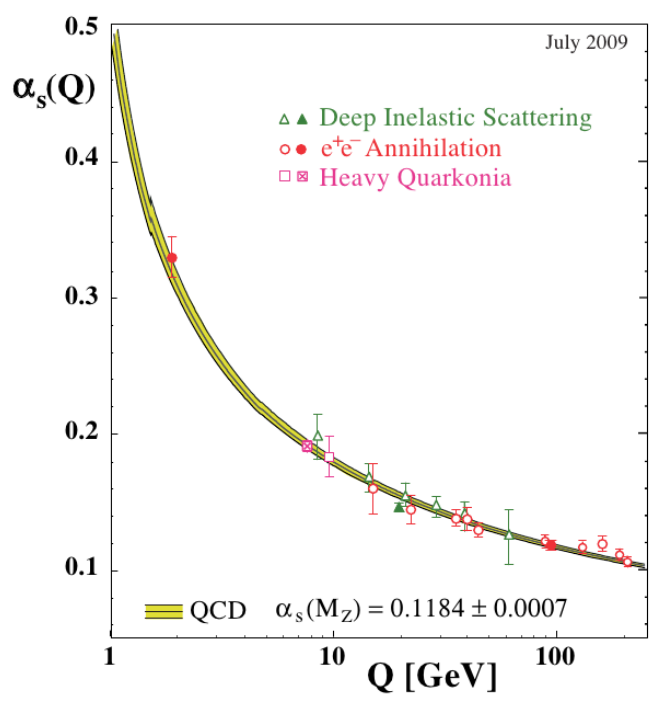

Figure 1.2.:2009 world average of running coupling $\alpha_{s}$ (from [14]).

Confinement and asymptotic freedom have relevant experimental consequences: quarks and gluons require interactions with high energy probes to be ejected from the nucleon, and they cannot be observed directly. What one detects instead of quarks and gluons are collimated showers of particles. These particles are the product of a series of steps, summarised as follows in the case of a hadronic collision event following Reference [15] and pictured in Figure 1.3:

1. Two hadrons (that can be seen as 'bags of partons' as in the parton model) collide with a large momentum transfer; 
2. Two partons (called incoming partons) from the hadrons collide and produce the hard process, leaving behind other partons (outgoing hadron remnants) and the products of other secondary collisions (multiple interactions).

3. Since electromagnetic and color charges interact, the incoming and outgoing partons can radiate (e.g. a quark can radiate a gluon). These processes can be described using perturbative QCD as outlined in Section 1.2.2.

4. When partons are sufficiently 'far' from each other, the coupling becomes strong and confinement plays a part. Being a non-perturbative process, the behaviour of the color force in this situation cannot be described using first principles in QCD. A simplified picture models the color force as that in a string with a linearly rising potential as charges become separated. The two extremities of the string are the opposite color charges. If the string is extended too much, it will break and create two additional color charges at the endpoints. If two opposite color charges are found close to each other, they will recombine and create a hadron that is observed in the final state. The creation of hadrons as a consequence of confinement is called hadronisation.

The products of the collision that are not directly identified with the hard products of the collision (hadron remnants, products of multiple parton interactions, radiation) are conventionally defined as underlying event. Further details on the simulation of these processes are given in Section 1.4.2.

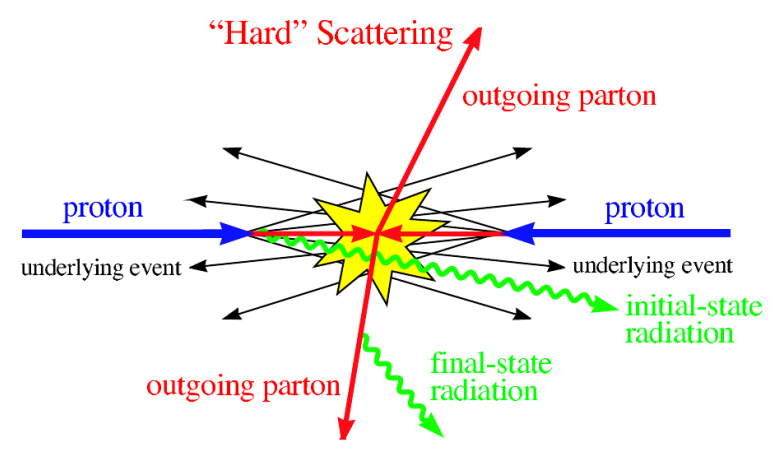

Figure 1.3.:Schematic representation of a parton-parton collision, leading to a $2 \rightarrow 2$ scattering event (from [16]). 


\subsubsection{Renormalisation, scale violations and parton distribution functions}

Asymptotic freedom allows QCD to be described using point-like constituents at sufficiently large energies. The first evidence of this behaviour was given by the SLAC-MIT experiments and interpreted by Feynman and Field using the so-called parton model [9]. On the other hand, the momentum scale introduced by renormalisation needed to be accommodated: the improved parton model was developed [17,18]. Starting from these ideas, the perturbative evolution of the quark and gluon behaviour can be predicted separately from the soft, non-perturbative physics, allowing for theoretical calculation of QCD processes.

The differential cross section for lepton-hadron $(l h)$ inelastic scattering (as in the SLAC experiments) can be parameterised starting from that of elastic scattering of fundamental particles. Equation 1.2 makes use of structure functions $F$ that encapsulate the lack of knowledge on the nucleon behaviour, the kinematics of the scattering (in terms of the momentum transfer $Q^{2}$ and of the functions $f(y)$ and $g(y)$ that depend on the kinematics of the scattering). It also introduces the variable $x$ that (at leading order) represents the fraction of hadron momentum taken by the struck quark:

$$
\frac{d^{2} \sigma(l h)}{d x d Q^{2}}=\frac{1}{Q^{4}}\left(f(y) x F_{1}\left(x, Q^{2}\right)+g(y) F_{2}\left(x, Q^{2}\right)\right)
$$

In comparison with the formula of the cross section for incoherent scattering, where each quark composing the hadron can be struck independently from the others, one finds that:

1. the structure function $F_{2}$ can be identified with the sum of the products of the quark charge squared $e_{i}^{2}$ and momentum distribution $x q_{i}(x)$ for all the quarks in the hadron. The $q_{i}(x)$ functions can be interpreted (at leading order of perturbation theory) as the probability densities of finding a quark with flavour $i$ carrying a fraction $x$ of the hadron momentum. The momentum distribution for a given quark or gluon is also called parton distribution function, or PDF.

$$
F_{2}^{l h}=\sum_{i}^{N_{q}} e_{i}^{2} x q_{i}(x)
$$


The independence of the structure function from momentum transfer $Q^{2}$ at fixed values of $x$ is known as Bjorken scaling.

2. the two structure functions are not independent if quarks have spin 1/2 (Callan-Gross equation).

The experimental verification of both approximate Bjorken scaling and the Callan-Gross relation in the measurement of the structure functions performed at SLAC shows that quarks in hadrons can be treated as quasi-free spin $1 / 2$ particles. This forms the basis of the quark parton model. Other structure functions, defined as combinations of the quark PDFs, have been derived and measured in data (for a review, see Chapter 5 of Reference [19]).

When integrating $F_{2}$ over all possible momentum fractions, only about half of the total hadron momentum is carried by quarks. Probability conservation requires that the area of the total momentum distributions in the proton is equal to unity (this requirement is also called momentum sum rule). This calls for the presence of gluons, later observed at the PETRA collider [20]. Gluons are responsible for quark interactions, since they are the carriers of strong force.

Conventionally, partons composing a hadron are divided between gluons, valence quarks and sea quarks. Valence quarks are responsible for the hadron's quantum numbers, while sea quarks are quark/antiquark pairs that can be generated thanks to quantum fluctuations.

Interactions among the partons lead to deviation from the naive parton model in terms of scaling violations, as shown in Figure 1.4 where experimental points at the same value of $x$ do depend on $Q^{2}$. Intuitively, an increase in $Q^{2}$ can be seen as an increase in the resolving power of the probe: if the internal structure of the hadron can be probed at smaller distances, then the number of partons 'seen' increases given that gluons can produce quark/antiquark pairs and quarks or antiquarks can radiate gluons. The DGLAP formalism [22] models these interactions through splitting functions and uses them to evolve perturbatively the renormalised parton densities that contain the $Q^{2}$ dependence. Given that quarks and gluons interact, the DGLAP equations that describe the change in quark, antiquark and gluon densities with $Q^{2}$ are coupled. The DGLAP formalism only gives information on the the evolution of the PDF, not on their shape, which is derived using a combination of the experimental data on the structure functions and an initial analytical form, as discussed in the next section. 


\section{H1 and ZEUS}

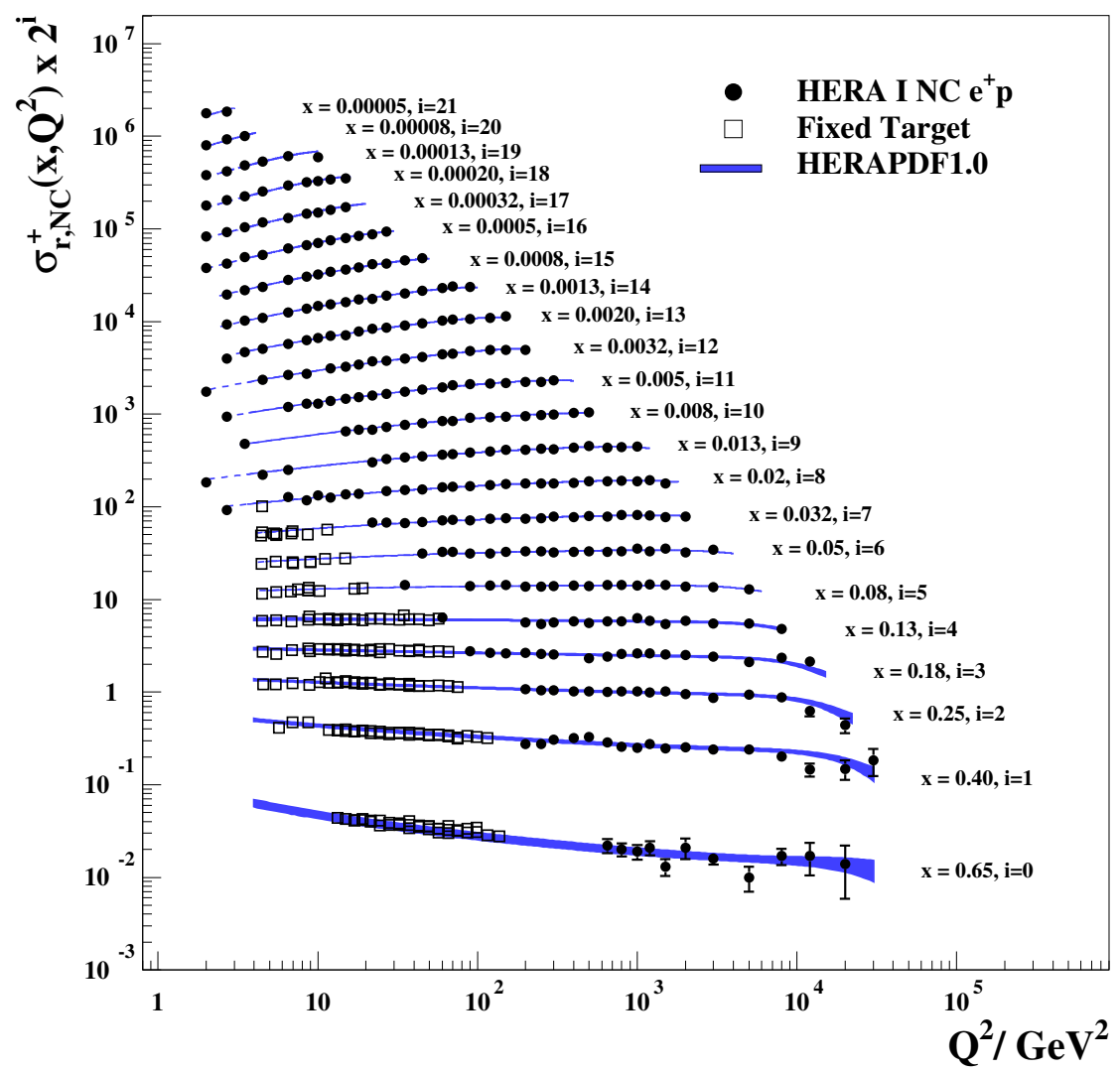

Figure 1.4.:Measurement of the structure function $F_{2}$ from the HERA experiments (Figure from [21]).

\section{PDF parameterisations}

Structure functions such as $F_{2}$, introduced in the previous section, can be measured experimentally. Parton distribution functions are not observable, but instead they are extracted using combinations of measurements for independent structure functions. Sum rules that constrain groups of PDF (e.g. on the consistency of the quantum numbers of the hadron) can also be used to infer information on parton distribution functions. Details on the extraction of PDF parameters are given in Chapter 7.

One essential ingredient for the extraction of parton distribution functions is the parameterisation of the PDFs at a starting scale. Since such a parameterisation is not predictable from first principles in QCD, simple considerations on the behaviour of the functions and assumptions on their shapes need to be introduced. For nucleons, the valence quark PDFs should be allowed to peak around $1 / 3$ (in the assumption that the three valence quarks will share equal fractions of 
the hadron momentum), with an additional smearing due to the intrinsic parton momentum and the presence of sea quarks. One also expects that sea quarks will grow for lower values of $x$ because of the divergent nature of QCD radiation and because gluons branch into $q \bar{q}$ pairs. Valence quarks will not have this behaviour because gluon interactions will not change the quark flavour. Furthermore, the parameterisations must respect the kinematic of the $x$ variable: 0 $<x<1$. This leads to the following basic parameterisations for the PDFs:

$$
x V(x)=A x^{B}(1-x)^{C}
$$

The behaviour at high $x$ can also be predicted using what is called the spectator model [23], while the the power-law behaviour for $x \rightarrow 0$ can be predicted using Regge trajectories [24].

The exact starting scale parameterisations used by the different groups that extract parton distribution functions vary, and those used for this thesis are detailed in Chapter 7.

\subsubsection{QCD Factorisation}

One of the reasons of the success of QCD as a predictive theory is that the short-distance component of the scattering process described by perturbative QCD can be separated from the non-perturbative long-distance component: this result is known as the factorisation theorem [25]. Factorisation implies that perturbation theory can be used to calculate the hard scattering cross section, while universal functions such as the PDFs can be included a posteriori to obtain the full theoretical prediction. This is shown in Equation 1.5 using the quantities in Figure 1.5 for a hard hadronic process:

$$
\begin{array}{r}
d \sigma_{\text {hard }}\left(p_{A}, p_{B}, Q^{2}\right)=\sum_{a b} \int d x_{a} d x_{b} f_{a / A}\left(x_{a}, \mu_{F}^{2}\right) f_{b / B}\left(x_{b}, \mu_{F}^{2}\right) \\
\times d \hat{\sigma}_{a b \rightarrow c d}\left(\alpha_{s}\left(\mu_{R}^{2}\right), Q^{2} / \mu_{R}^{2}\right) .
\end{array}
$$

$f_{a / A}$ and $f_{b / B}$ are the parton momentum densities for the two interacting partons $a, b$ with respect to hadrons $A, B$. Since factorisation is a byproduct of a procedure that absorbs singularities into physical quantities in the same fashion as renormalisation, a new scale $\mu_{F}^{2}$ is introduced. When truncating calculations at a given order, the uncertainties due to the choice of scale need to be calculated in order to account for higher order terms. 
Factorisation is related to the connection of inclusive hadronic observables to the underlying kinematics of the hard scattering of quarks and gluons, even when the final signature is influenced by parton distribution functions, fragmentation and hadronisation.

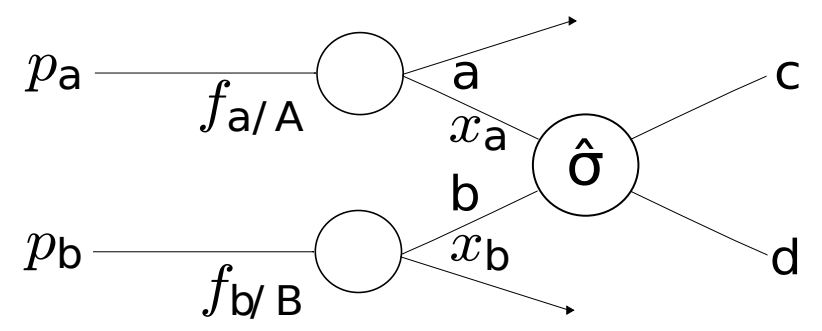

Figure 1.5.:Schematic illustration of hard hadronic process (Figure from [19]).

Factorisation and the comparison of experimental data from different processes indicate that the parton distribution functions are universal (i.e. they do not depend on the physics process). They can be derived from different physics processes and then used to derive full theoretical predictions independently from the calculation of the hard scattering cross section. The universality of parton distribution functions is confirmed by the comparison of the extraction of the structure functions from different processes as mentioned in Section 1.2.2.

\subsection{Infrared safety in inclusive processes: jets}

QCD cross sections diverge when calculating processes that involve the radiation of infinitely soft particles (infrared divergencies) and when radiation happens at small angle (collinear divergences). However, infrared divergences cancel by virtue of the Kinoshita-Lee-Nauenberg theorem [26,27]. The practical consequence of this theorem is that observables that are inclusive enough to be insensitive to processes that distinguish between different numbers of partons are infrared safe and are not affected by infrared divergences. This is mirrored by the behaviour of experimental measurements: detectors have a finite spatial resolution and will not be able to resolve the products of separate partons.

If the objects that represent partons in the final state have a well defined behaviour both in theory and experiments, then the parton kinematics will be reflected in the kinematic of the final state object. This is where the definition of jets becomes indispensable for QCD measurements. A jet can be naïvely seen as a group of collimated particles generated by the hadronisation of a parton in the scattering process, as shown schematically in Fig. 1.6.

The concept of jets was introduced even before QCD was formalised (see for example Reference [28]). A rigorous definition of jets for use as a connection between perturbative QCD and observable hadronic final state was introduced by Sternman and Weinberg [29]. Given that 


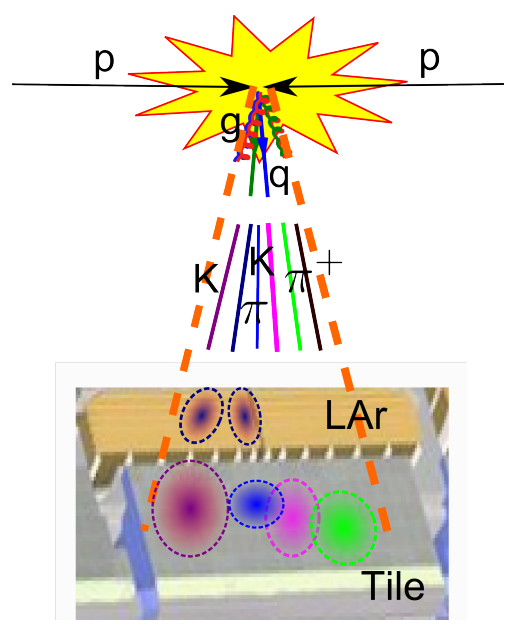

Figure 1.6.:Development of a jet: collimated particles from the hadronisation of quarks and gluons deposit energy in the ATLAS calorimeters, as described in Chapter 2.

thanks to momentum conservation two partons with sufficiently high transverse momenta will be produced back to back, one can define a jet cross section by including all partons and their radiation if they are found in two cones of opening angle $\delta$. These cones will only leave out a finite amount of energy $\epsilon$. The definition of boundaries for the integration in terms of $\delta$ and $\epsilon$ allows for a finite cross section. A similar algorithm can also be used when dealing with the two collimated sprays of particles produced by the hadronisation of the partons. Different infrared and collinear safe algorithms have since been introduced, but what remains of this interpretation is the requirement of using the same jet finding algorithm in theory and experiment to be able to compare theoretical prediction to data. More details on the definition of jets and the algorithms used for jet finding are given in Chapter 3 .

\subsubsection{Inclusive jet production}

The inclusive jet cross section is a standard tool to test perturbative QCD at hadron colliders, spanning over numerous orders of magnitude in terms of the jet transverse momentum. The experimental measurement does not present particular difficulties in terms of background subtraction given that jets are the predominant hard process produced in the hadron-hadron collisions.

Jets are dominantly produced through scattering of two partons (gluon-gluon, quark-gluon or quark-antiquark) leading to two partons in the final state. As mentioned in Section 1.2, asymptotic freedom and factorisation allow the calculation of theoretical predictions for QCD processes using perturbation theory. The difficulty of theoretical calculations increases with the order of perturbation, corresponding to the number of partons involved. At leading order 
only two initial and final state partons are involved. For each increasing order of perturbation theory, one parton needs to be added to the $2 \rightarrow 2$ process, either as a 'leg' or as a 'loop', and the diagrams will interfere in the calculation. The theoretical prediction for the inclusive jet cross section is known at next to leading order (NLO) in perturbation theory.

\subsubsection{Motivations to measure the inclusive jet cross section}

Jets are a necessary ingredient for many analyses at a hadron collider. Measuring the inclusive cross section requires a detailed understanding of jets and the related systematic uncertainties. Since the theory/data comparison for the inclusive jet cross section in measurements prior to the one detailed in this thesis showed excellent agreement, as in References [30] and [31], an early data/theory comparison can be performed in a kinematic region where agreement has been previously found to ensure that jets in ATLAS are well understood.

Furthermore, a measurement of the jet cross section with the data collected by the ATLAS detector in the first year of operation of the Large Hadron Collider spans kinematic regions that were previously unexplored, and it can be used as a more stringent test of perturbative QCD over phase space. If the experimental and theoretical uncertainties are small enough and well understood, the measurement can also be used to constrain the parton distribution functions exploiting the extended kinematic range, especially the gluon distribution at values of $x$ up to 0.2. This topic will be developed further in Chapter 7.

\subsection{Theoretical tools}

A theoretical prediction is fully comparable to data when corrections are applied to the calculation to simulate additional orders in perturbation theory and non perturbative effects, and after all detector-specific effects have been removed from the experimental measurement. The tools used to calculate the NLO theoretical prediction, for the extraction of jet calibration parameters and for the theory/data comparison of the measurement of the inclusive jet cross section detailed in this thesis are listed in the following paragraphs.

\subsubsection{NLO calculation software and convolution with PDFs}

The next to leading order calculation for the hard process is performed using the NLOJET ++ 4.1.2 [32] software. This result needs to be convoluted with the parton distribution functions at given renormalisation and factorisation scales: this is achieved with the APPLGrid software [33]. APPLGrid allows for a posteriori inclusion of PDF and process scales, so that the time-consuming 
calculation of the next to leading order coefficients only need to be evaluated once. This is particularly useful when systematic uncertainties derived from variations of the PDFs, scales and strong coupling constant need to be estimated.

\subsubsection{Parton shower Monte Carlo generators}

Monte Carlo event generators such as Pythia [34] and AlPGEn [35] are employed to simulate inclusive QCD jet events from proton-proton collisions when the information on the particles entering the detector after hadronisation is needed.

These generators simulate the entire QCD event, using various models to simulate the non-perturbative steps mentioned in Section 1.2.1. No full calculation beyond leading order is employed in the generators that are normally used, although the ALPGEN generator is able to provide matrix elements for up to 6 additional partons as 'legs' of the diagram. After the hard scattering $(\mathbb{2} \rightarrow \mathscr{2}$, or $2 \rightarrow n)$ is simulated starting from the matrix element calculation that includes the convolution with a PDF of choice, subsequent radiation is simulated using a series of probabilities for partons to split. This approach is called parton shower, and it needs to be matched to the matrix element calculation to avoid double counting of radiation generated by both the hard scattering and by the parton shower. The splittings are iterated until a cut-off scale is reached (usually $1 \mathrm{GeV}$ ), and at that point hadronisation will start. These generators will have specific models (or employ external plug-ins) for hadronisation and multiple parton interactions, whose parameters can be tuned using specific data.

The Monte Carlo generators used in the inclusive jet analysis are listed below, together with their main characteristics and choices of PDF and tunes.

PyтHIA simulates non-diffractive proton-proton collisions using a $2 \rightarrow 2$ matrix element at leading-order of the strong coupling to model the hard subprocess, and use parton showers to model additional radiation in the leading-logarithmic approximation using $p_{T}$-ordering [36]. The hadronisation model used is an evolution of the string model described in Section 1.2.1 (Lund string model [37]). Multiple parton interactions are also simulated within Pythia [36]. The PDF used for the Pythia samples is the modified leading order set MRST LO* [38]. Modified leading order PDFs can be used in combination with a leading order generator to obtain a result qualitatively similar to what one would obtain with a next to leading order calculation ${ }^{2}$.

\footnotetext{
${ }^{2}$ This statement is currently being tested in a number of measurements at LHC energies.
} 
ALPGEN is a leading order matrix-element generator for hard multi-parton processes in hadronic collisions [35]. It is interfaced to the HERWIG generator to produce angular-ordered parton showers in leading-logarithmic approximation [39]. Parton showers are matched to the matrix element with the MLM matching scheme [40]. The hadronization is simulated in HERWIG [39] using the cluster model [41] where gluons split in $q \bar{q}$ pairs that are subsequently regrouped into colorless clusters to produce hadrons. Multiple parton interactions are modelled using Jimmy [42]. The PDF used for the AlPGEN samples is CTEQ6L1 [43].

HeRwiG ++ [44] is a multipurpose event generator built on the experience of HeRWIG. It simulates the full hadronic interaction in a similar fashion as PYTHIA, but using angular ordering in the generation of the parton shower and specific hadronisation and multiparton interactions models [45]. The MRST LO* PDF set is used in combination with the HERWIG++ generator.

The parameters used for tuning the underlying event models in the PYTHIA and HERWIG event generators have been derived from minimum bias measurement in ATLAS data $[46,47]$ and are denoted as ATLAS MC10 tune.

\section{Nominal Monte Carlo sample}

The baseline Monte Carlo sample used in this analysis for the calibration and derivation of the jet energy scale and its uncertainty is generated with PYTHIA and will be referred to as nominal sample in the following. The full sample ${ }^{3}$ is composed of 12.6 million simulated PYTHIA QCD jet events. Due to the steeply falling jet cross section, the samples have been generated in 9 bins of transverse momentum of the outgoing partons in the $2 \rightarrow 2$ hard scattering process $\left(\hat{p}_{T}\right)$, covering the range $8 \mathrm{GeV}-3.5 \mathrm{TeV}$. Each sub-sample is weighted according to its cross section to form the final event sample. The sample used for the theory/data comparisons and for the derivation of the non perturbative corrections that need to be applied to the NLO calculation includes the nominal PYтHIA sample, complemented by a minimum bias sample of approximately 20 million events for jets with $\hat{p}_{T}$ below $8 \mathrm{GeV}$. Events with $\hat{p}_{T}$ below $17 \mathrm{GeV}$ are taken from the minimum bias sample, while events above this threshold are taken from the nominal sample mentioned above.

The PythIA QCD jet sample has been validated with data collected by the ATLAS detector at $\sqrt{s}=900 \mathrm{GeV}$ and $\sqrt{s}=7 \mathrm{TeV}$ [48-55]. Jets measured in ATLAS are reasonably well described by the Monte Carlo simulation, before and after their calibration, as shown in Figure 1.7 .

\footnotetext{
${ }^{3}$ The calibration has been derived on a smaller sample of 3.6 million events.
} 


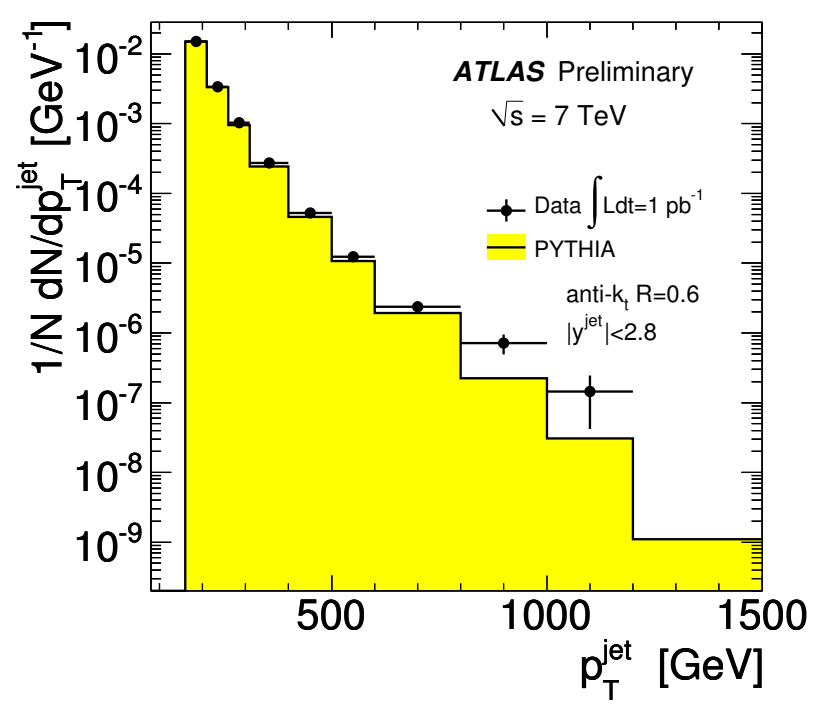

Figure 1.7.:Observed inclusive jet $p_{T}$ distribution (black dots) for anti- $k_{t} R=0.6$ jets (as defined in Chapter 3) with $p_{T}>160 \mathrm{GeV}$ and pseudorapidity $|y|<2.8$ compared to the Monte Carlo prediction from the nominal sample (yellow histogram). The distribution is normalized to unity and only statistical uncertainties are included. 


\section{Chapter 2.}

\section{The ATLAS Experiment}

The study of new phenomena in particle physics at the Terascale, the discovery of the Higgs boson and further measurements of Standard Model quantities are among the main goals of the experiments located on the Large Hadron Collider (LHC), at CERN in Geneva. At the LHC, proton beams with a centre of mass energy of $7 \mathrm{TeV}$ collide up to every $50 \mathrm{~ns}$ at the four interaction points, where the experiments are installed. The ATLAS (A Toroidal LHC ApparatuS) experiment is a general purpose detector located at the Interaction Point 1 on the French-Swiss border, and has been recording collision data at $7 \mathrm{TeV}$ starting from the Spring of 2010.

This Chapter outlines the layout of the ATLAS detector (Section 2.2) and its subsystems (Sections 2.3 and 2.4). Given their relevance for the measurements described in this thesis, the ATLAS calorimeters are described in more detail in Section 2.5. Section 2.6 describes the simulation infrastructure of the ATLAS detector, with a particular focus on hadronic interactions within the calorimeters. Finally, the computing infrastructure, the data used for the measurement and the trigger system used to select relevant events are described in Sections $2.7-2.9$.

\subsection{CERN and the Large Hadron Collider}

CERN, the European Organization for Nuclear Research, is the largest international particle physics laboratory in the world. It is located near Geneva, at the border between Switzerland and France. The current flagship project at CERN is a particle accelerator called the Large Hadron Collider (LHC). The LHC accelerates and collides proton beams at a centre of mass energy of $7 \mathrm{TeV}$. It has taken almost 20 years of design, development, construction and testing, and it delivered its first collisions in November 2009, achieving the world-record energy of $7 \mathrm{TeV}$ 
in March 2010. The products of the LHC collisions are detected by four large scale experiments situated at the collision points, as shown in Figure 2.1.

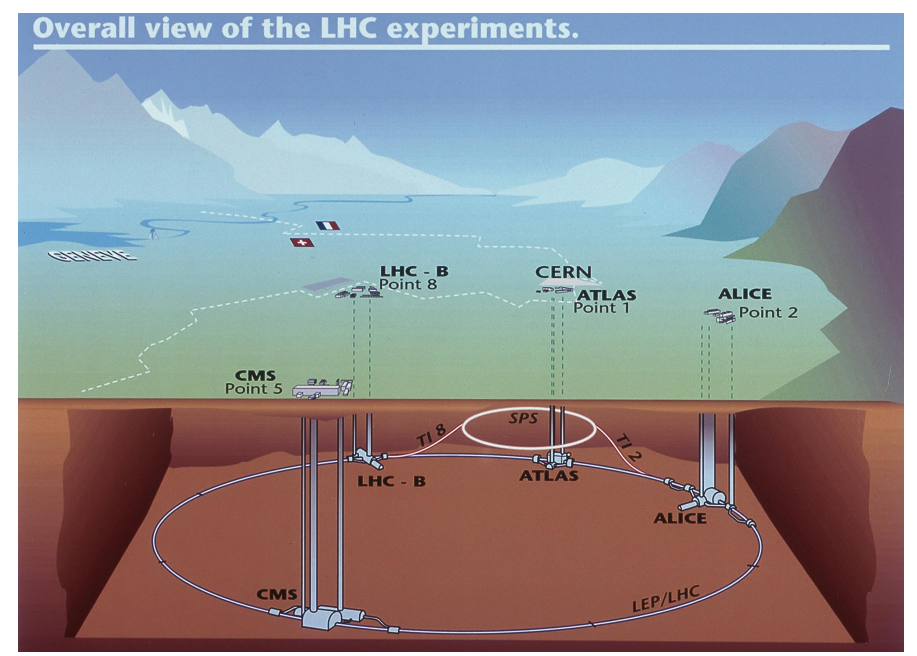

Figure 2.1.:The four main experiments located around the LHC ring. The SPS, the final link in the pre-acceleration chain, and its connection tunnels to the LHC are also shown. From [56].

\subsubsection{Running conditions of the LHC accelerator}

The ATLAS detector records proton-proton collision events delivered by the Large Hadron Collider. Bunches of protons circulate in separate vacuum tubes in opposite directions within the beam pipe, and are brought together to collide by quadrupole magnets in the four interaction points. One of the figures of merit of the accelerator is the luminosity, since it connects the number of events per unit of time $d N / d t$ to the cross section $\sigma$ of a given process: $d N / d t=\mathcal{L} \sigma$. The physics program at the Large Hadron Collider includes searches for rare physics processes (with a small cross section) such as the Higgs boson and supersymmetric particles: it is therefore important to maximise the delivered luminosity in order to be able to observe such events.

An increase in the luminosity can be achieved by squeezing the beams and reducing their transverse size, or by increasing the number of colliding protons per bunch or the number of circulating bunches. The first two effects lead to an increase of proton-proton interactions within the same bunch (in-time pile-up), while the latter two lead to multiple interactions from different bunches during the time taken for the detector to process a single event (out-of-time pile-up). Out-of-time pile-up can also be produced by the circulation of multiple subsequent bunches (bunch train). The very first data delivered by the LHC had a negligible number of multiple interactions until May 2010, when the accelerator optics was optimised to decrease the size of the beams at the interaction point: this lead to an increase in the number of events with more than a single proton-proton interaction to about $10 \%$. The number of protons per 
bunch was increased throughout the course of the summer, leading to a rise in the fraction of events with in-time pile-up that continued up to November 2010, when the average number of interactions was more than three [57]. This is shown in Figure 2.2.

The instantaneous luminosity delivered by the LHC is estimated using ATLAS subdetectors built for this purpose, as described in detail in References $[58,59]$. The systematic error on the integrated luminosity for the full 2010 dataset is $3.4 \%$.

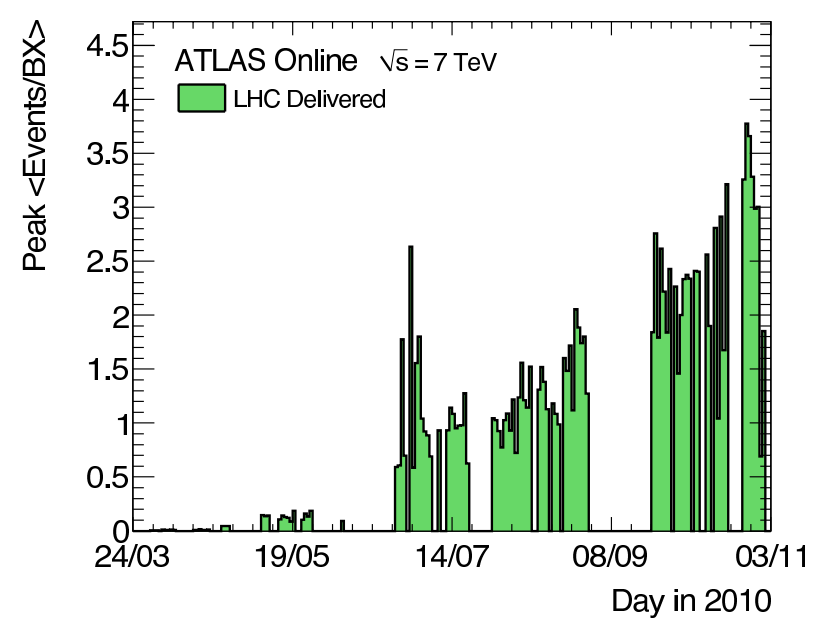

Figure 2.2.:The number of interactions per bunch crossing (BX) as measured online by the ATLAS luminosity detectors. In order to calculate the equivalent mean number of interactions, an inelastic cross-section of $\sigma_{\text {inel }}=71.5 \mathrm{mb}$ is assumed. Figure from [57].

The LHC design luminosity is $\approx 10^{34} \mathrm{~cm}^{-2} \mathrm{~s}^{-1}$, leading to 22 simultaneous proton-proton interactions and to more than 1000 particles being produced within one event in the central detector region, and the maximum instantaneous luminosity obtained in 2010 is $\approx 2 \cdot 10^{32}$ $\mathrm{cm}^{-2} \mathrm{~s}^{-1}$. These conditions impose strong requirements on the radiation hardness and granularity of the detectors: the design of the ATLAS detector that can withstand these is described in Section 2.2. The high luminosity combined with the proton-proton inelastic cross section of 71.5 $\mathrm{mb}$ [58] requires a fast system for the selection of interesting events: the ATLAS trigger system is described in Section 2.9 .

\subsection{Overview of the ATLAS detector and coordinate system}

The ATLAS experiment is located at Point 1 of the LHC ring, and it is a collaboration consisting of more than 2900 physicists from 172 different Institutes and Universities throughout the world. Its layout is shown in Figure 2.3. The ATLAS detector is a general purpose experiment that has 
been built to perform well in the high-intensity and high energy hadronic environment of the Large Hadron Collider and have a wide physics program that includes precise Standard Model measurements, searches for the Higgs boson, for supersymmetric signatures and for physics beyond the Standard Model.

The ATLAS detector consists of a tracking system (inner detector, or ID in the following) in a $2 \mathrm{~T}$ solenoidal magnetic field up to a pseudorapidity $|\eta|<2.5$, sampling electromagnetic and hadronic calorimeters up to $|\eta|<4.9$, and muon chambers in a toroidal magnetic field provided by air-core toroids. A detailed description of the ATLAS experiment can be found in [60] and its subsystems are described in the sections below.

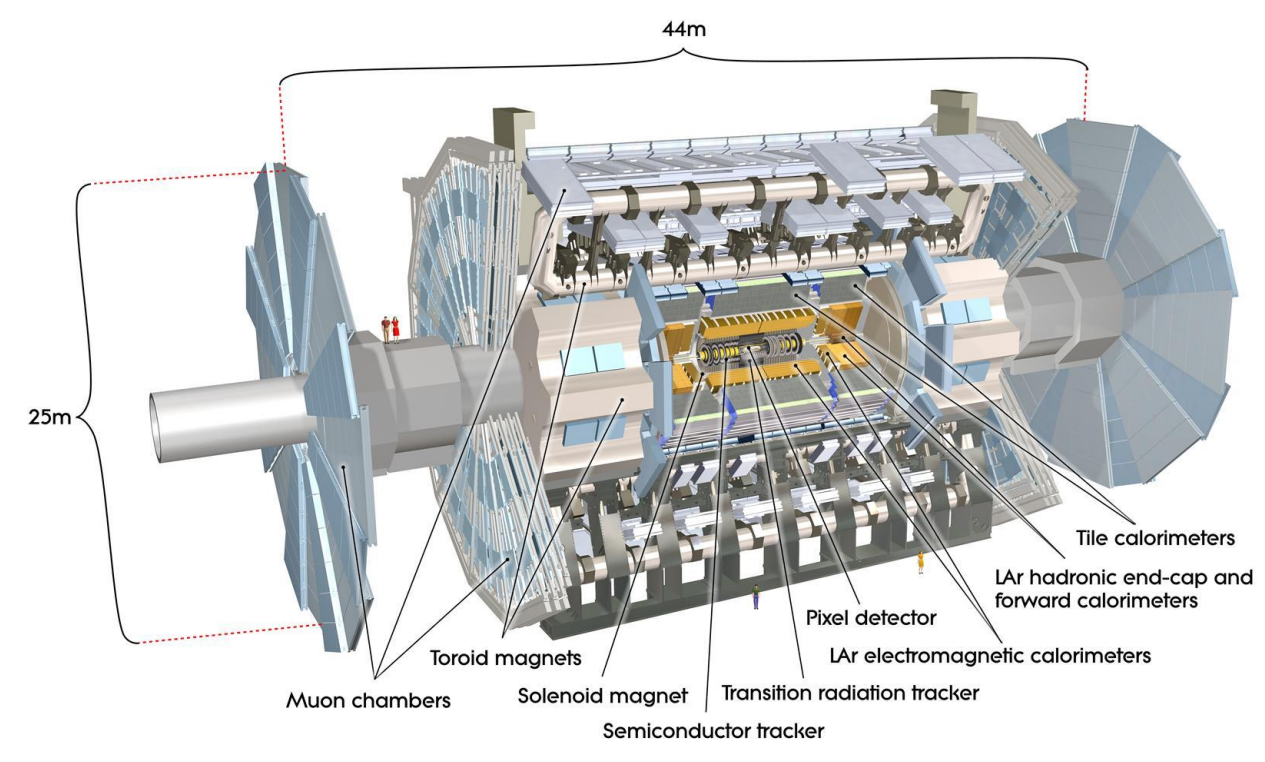

Figure 2.3.:A detailed computer-generated image of the ATLAS detector and its subsystems. Figure from [61].

The ATLAS Coordinate System is a right-handed system with the $x$-axis pointing to the centre of the LHC ring, the $z$-axis following the beam direction and the $y$-axis pointing upwards. The azimuthal angle $\phi=0$ corresponds to the positive $x$-axis and $\phi$ increases clock-wise looking into the positive $z$ direction. The pseudorapidity $\eta$ is an approximation for the rapidity $\mathrm{y}$ in the high energy limit, and it is related to the polar angle $\theta$ as $\eta=-\ln \tan \frac{\theta}{2}$. The $R$ coordinate is a measure of the radial distance with respect to the interaction point and it follows the direction of the $y$-axis. In the following, the central detector region (generally up to $\eta<1.5$ ) is referred to as barrel, the more forward region (up to $\eta<2.5$ unless otherwise noted) as endcap and the forwardmost pseudorapidities are simply called forward region. 


\subsection{The ATLAS inner detector system}

The ATLAS Inner Detector system consists of high-resolution silicon detectors (pixels and strips) surrounding the interaction point to locate the interaction vertex, measuring displaced vertices from long-lived particles and measuring precisely the track momentum. The tracking detectors are arranged in concentric cylinders around the beam axis in the barrel region, while disks are used in the endcap region, and they are located within a $2 \mathrm{~T}$ magnetic field generated by a superconducting solenoidal magnet. The coverage of the tracking detectors is up to $|\eta|<2.5$. Figure 2.4 (a) displays the layout of the inner detector system.

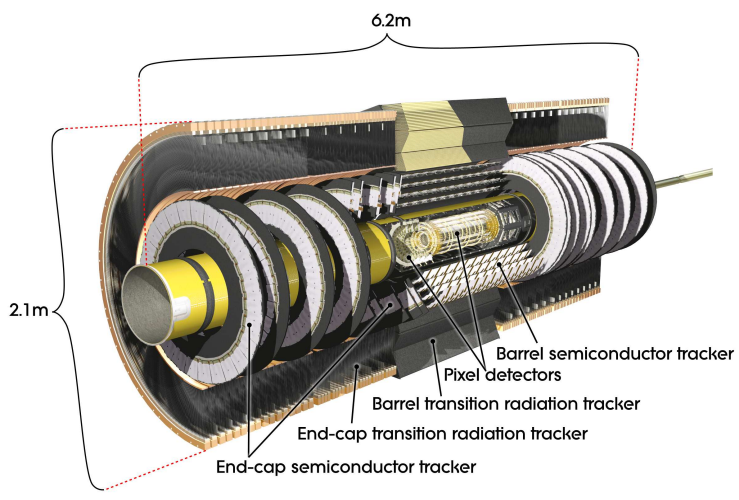

(a)

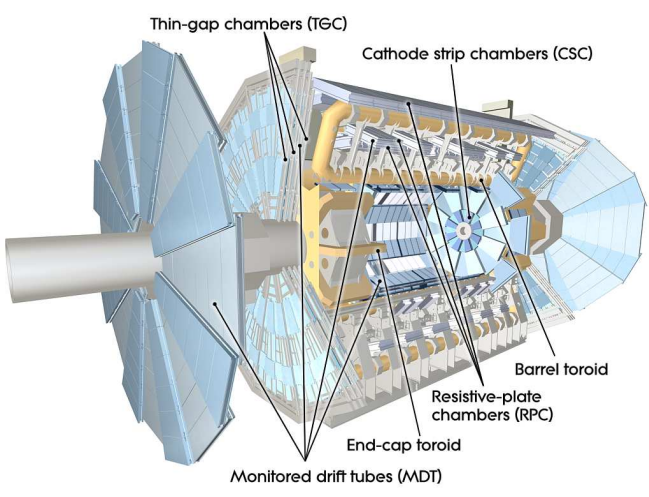

(b)

Figure 2.4.:Computer-generated images of the ATLAS inner detector system (a) and muon detectors (b). Figures from [61].

The core components of the ATLAS pixel detector are semiconducting pixels, assembled in silicon wafers called sensors. The SemiConductor Tracker (SCT) consists of modules formed by microstrip semiconductor strips arranged parallel to the beam pipe in the barrel region and radially in the endcaps, providing a measurement in the $R-\phi$ coordinate. Pairs of sensors are glued together at a $40 \mathrm{mrad}$ stereo angle to allow for a measurement of the $\mathrm{z}$ coordinate. The ATLAS Transition Radiation Tracker (TRT) is comprised of straw tubes filled with a Xenon-based gas mixture to provide continuous $R-\phi$ information and particle identification.

The tracking algorithms use information from all the inner detector subsystems. The raw data are first converted into three dimensional space points in the pixel and SCT, and into drift circles $^{1}$ in the case of the TRT. The track finding and vertexing software [62] is then employed to find prompt tracks that originate from vertices near the interaction point. Track candidates are built from space points in the silicon layers, fitted and then extended to the TRT using the

\footnotetext{
${ }^{1}$ The radius of a drift circle corresponds to the distance of closest approach of the charged particle to the wire as estimated from the time needed for drift electrons to reach the wire.
} 
full ID information. A dedicated vertex finder identifies the primary vertex as that with the highest sum of $p_{T}$ of the tracks pointing to it, normalized by the number of tracks.

\subsection{The ATLAS muon system}

Muons are deflected by the large air core toroids placed outside the calorimeter system, three in the barrel and two in the endcap. The toroids provide a magnetic field generally orthogonal to the muon direction, and their air core minimises the deflections due to multiple scattering. The ATLAS muon system triggers on and measures the muon momentum: chambers with higher precision (Monitored Drift Tubes and Cathode Strip Chambers) are employed for precise measurements of muons up to $|\eta|<2.7$ and coarser chambers (Resistive Plate Chambers and Thin Gap Chambers) are used for selecting interesting events containing muon candidates.

\subsection{The ATLAS calorimeter system}

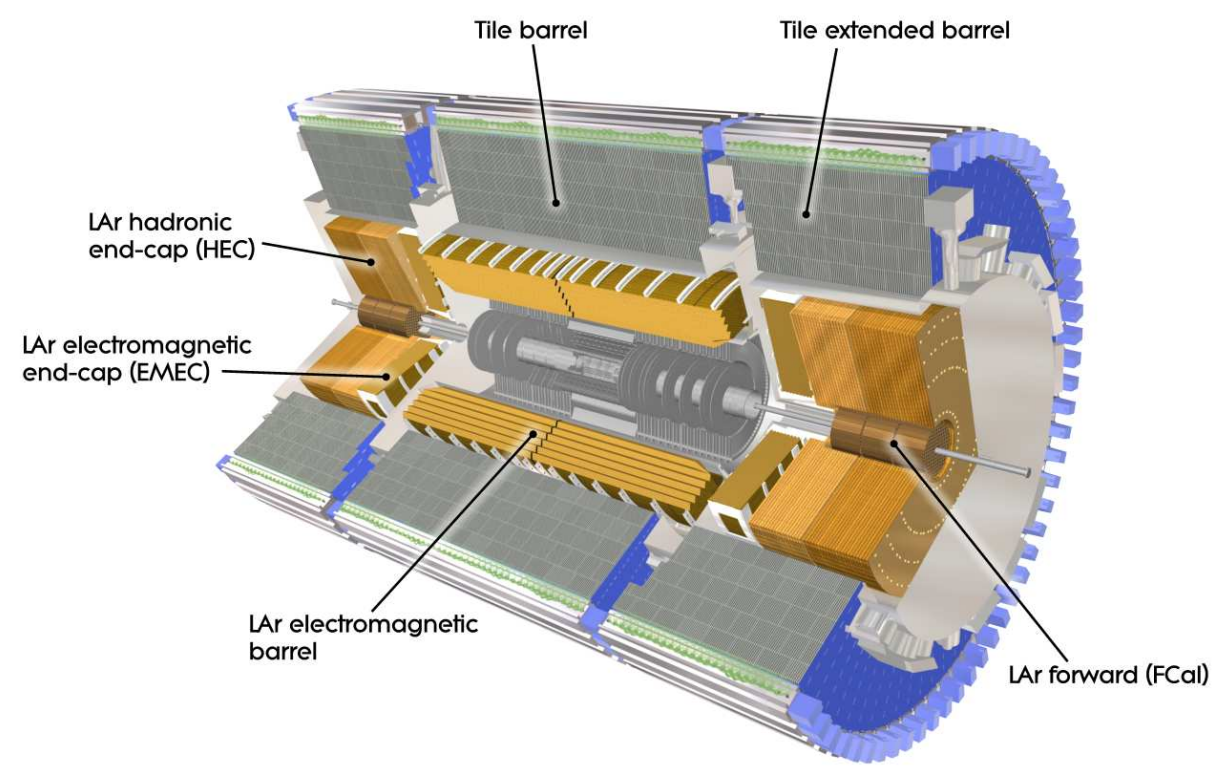

Figure 2.5.:A computer-generated image of the ATLAS calorimeter system. Figure from [61].

The ATLAS calorimeter system (Figure 2.5) aims to measure the energy and position of particles through the absorption of their energy deposits in the calorimeter material. Particle cascades (showers) are produced in this process. The ATLAS calorimetry system employs a sampling technique (sandwiching active and passive material) that separates the absorption from the energy measurement and allows for a more compact design with an almost complete containment of the showers. ATLAS includes an electromagnetic and a hadronic calorimeter, 
with different characteristics in order to account for the different properties of electromagnetic and hadronic showers.

ATLAS calorimeters are non compensating: the hadron response is lower than the response to electromagnetically interacting particles. While the energy of an electromagnetic shower is usually fully detected in the electromagnetic calorimeter, not all the energy of a hadronic shower can be detected in the calorimeters: this is mainly due to invisible energy from the nuclear breakdowns and excitation, and partly to invisible particles escaping detection $(\mu, \nu)$ [63]. Techniques to restore the correct energy scale for hadronic particles are described in Section 3.2. The starting point for the calibration of all energy deposits, hadronic or electromagnetic, is a measurement of the energy deposited in the single readout cell at the electromagnetic scale (also called EM-scale in the following). The ATLAS calorimeters and the procedure to set the electromagnetic scale are described in the following sections.

\subsubsection{Electromagnetic calorimeters}

The ATLAS electromagnetic calorimeter (also called $L A r$ calorimeter) employs liquid argon as the active material and lead as absorber. Charged particles traversing the calorimeter ionise the liquid argon, and the resulting electrons drift towards the copper electrodes in the read out cells thanks to the presence of an electric field. The two LAr calorimeter barrels span up to $|\eta|=1.475$, while the coverage of the endcaps is $1.375 \leq|\eta|<3.2$ in two coaxial wheels $(1.375 \leq|\eta|<2.5$ and $2.5 \leq|\eta|<3.2)$. The crack region between $1.375 \leq|\eta|<1.52$ is affected by additional material needed to instrument and cool the inner detector, and it is normally excluded from analyses that require a precise measurement of electrons.

The LAr calorimeter barrel has an accordion structure as shown in Figure 2.6, designed to avoid azimuthal cracks, provide full $\phi$ symmetry for particles traversing it and allow for a fast readout. The liquid argon is located in the gaps between the 1024 accordion absorbers, leading to a drift time of approximately $450 \mathrm{~ns}$ under the influence of the $2 \mathrm{kV}$ electric field. Since the drift velocity enters the energy measurement ${ }^{2}$, the detector conditions that influence it such as high voltage and liquid argon temperature and density need to be continuously kept under control to reduce the fluctuations in the energy measurement. The width of the gap is $2.1 \mathrm{~mm}$ in the barrel and varies with pseudorapidity in the endcap, so the high voltage needs to vary accordingly to obtain a calorimeter response independent from pseudorapidity. The

\footnotetext{
${ }^{2}$ The drift time $T_{d r i f t}$ is connected to the width of the gap $w_{\text {gap }}$ and the drift velocity $v_{\text {drift }}$ as $T_{d r i f t}=$ $w_{\text {gap }} / v_{\text {drift }}$. In turn, the time dependence of the ionisation current $I$ that is input to the energy measurement can be described using a baseline current $I_{0}$ that again depends on the drift velocity: $I(t)=I_{0}\left(1-t / T_{\text {drift }}\right)$.
} 


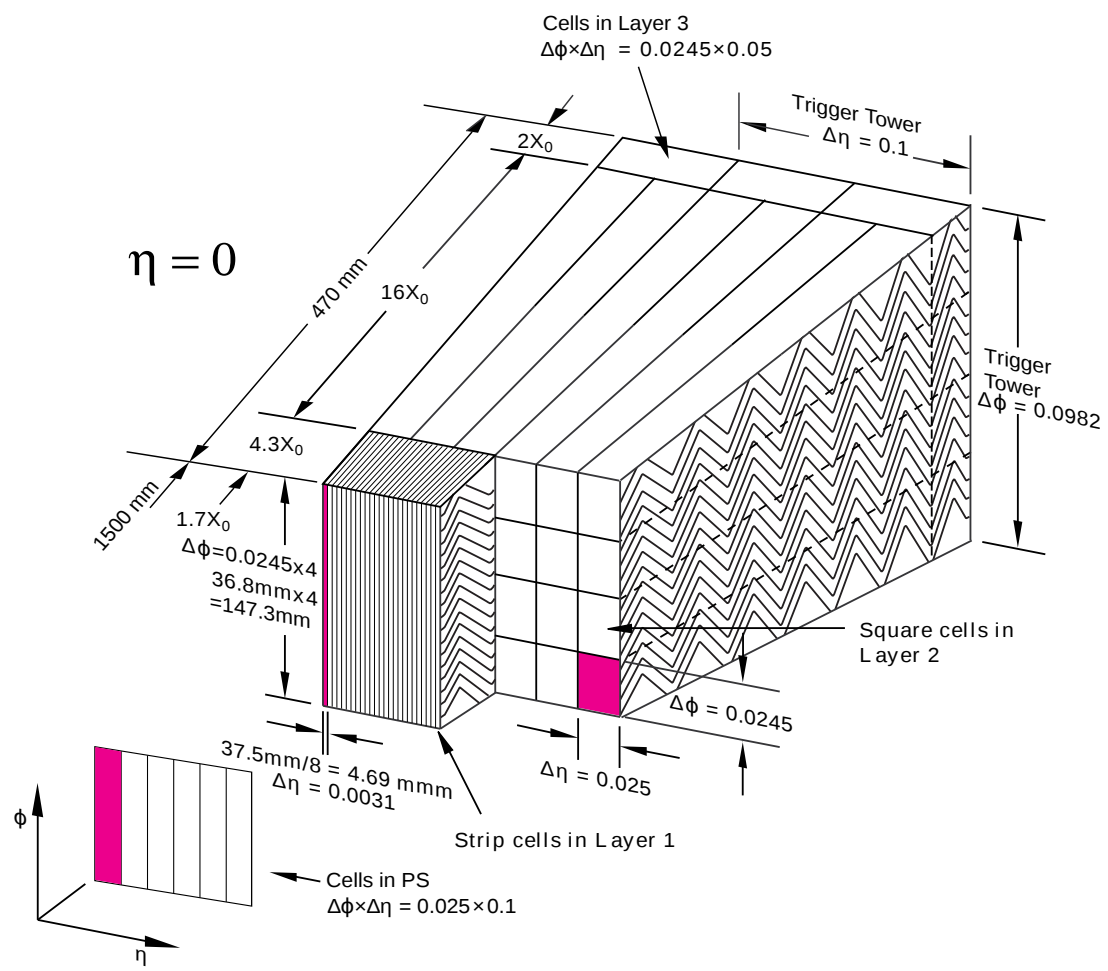

Figure 2.6.:Sketch of the structure of the LAr calorimeter barrel, showing the accordion structure and the granularity of the different layers. Figure from [61].

electromagnetic calorimeters are located in cryostats, since liquid argon needs to be kept at a temperature of about $88 \mathrm{~K}$.

The LAr calorimeter barrel is divided into three layers, as shown in Figure 2.6. The first layer, finely segmented in strips of $\Delta \eta \times \Delta \phi=0.0031 \times 0.098$, is used to reconstruct the $\eta$ position of the electromagnetic shower and provide information on particle identification. The second layer collects the largest fraction of energy deposited by the shower in its $\approx 16 X_{0}$, and the third layer only collects the shower tail. The electrodes in the second and third layer are grouped in towers of $\Delta \eta \times \Delta \phi=0.0245 \times 0.025$ and $0.0245 \times 0.05$ respectively. The total number of radiation lengths traversed by a particle in the electromagnetic calorimeter ranges from 22 to 33. The first wheel of the LAr endcaps is segmented in three layers with a granularity equal to that of the barrel, while the second wheel (for larger pseudorapidities) has a coarser granularity that varies a function pseudorapidity.

The distribution of material upstream of the calorimeters in terms of radiation lengths is shown in Figure 2.7. In order to correct for the energy lost by incident particles traversing this material, a presampler detector is used for $|\eta|<1.8$. The presampler is composed of an active layer of LAr with $1.1(0.5) \mathrm{cm}$ thickness in the barrel (endcap). A scintillator slab is also placed 
in the gap between barrel and endcap in the boundary between the barrel and endcap cryostat $(1.0 \leq|\eta|<1.6)$.

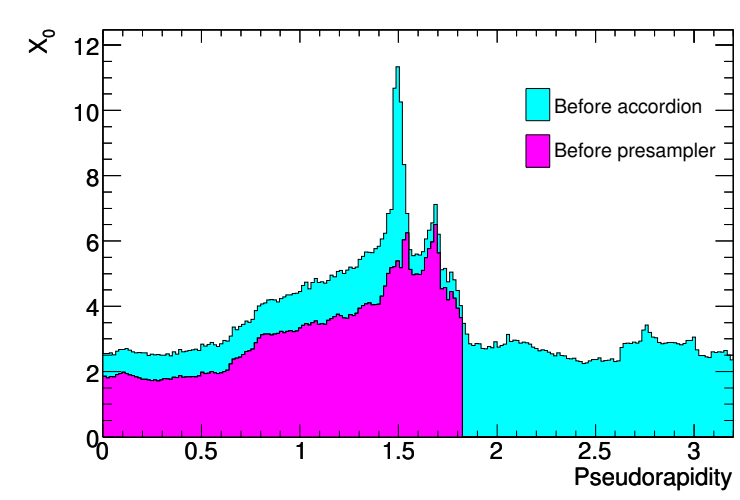

(a)

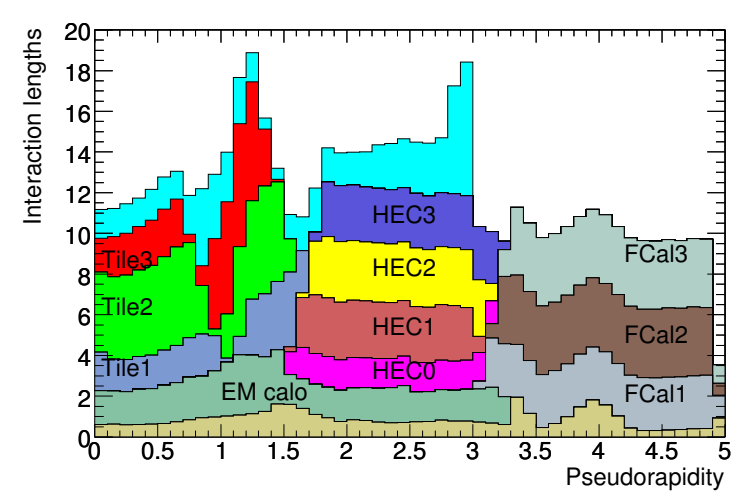

(b)

Figure 2.7.:Figure (a) shows the amount of material upstream the LAr calorimeter barrel (teal) and the presampler (magenta). The cumulative amount of material in units of interaction length in front and within the ATLAS calorimeters, and the total amount of material in front of the first active layer of the muon spectrometer (up to $|\eta|<3.0$ ) is shown in Figure (b). Figures from [61].

\section{Liquid argon (LAr) calorimeter calibration and uncertainty on the EM energy scale}

The analog signal produced by the ionisation of charged particles is amplified, shaped and sampled every $25 \mathrm{~ns}$. If the event is retained by the trigger signal (see Section 2.9), a number of samples (5 or 7 ) are digitised and sent to the off-detector electronics for calibration. The full electronic calibration procedure to convert the raw signal to a pulse shape in ADC counts such as the one in Figure 2.8 and to extract the visible energy deposited in each cell is described in detail in References [64-66].

Electron and photon candidates are reconstructed gathering calorimeter cells in clusters, using a sliding window algorithm [69] and combining energies deposited in each layer. The cluster energy and position needs to be determined precisely starting from the visible energy deposited in the cells and taking into account the shower development in the sampling calorimeters, the energy deposited upstream the calorimeter (using information from the presampler), the leakage outside the calorimeters and the modulations of the energy in $\eta$ and $\phi$ due to the accordion geometry. These factors and the calibration constants for the electronics (including corrections for known high voltage problems) have been derived and validated using electron and muon test beams and Monte Carlo simulation [65, 70-76]. 


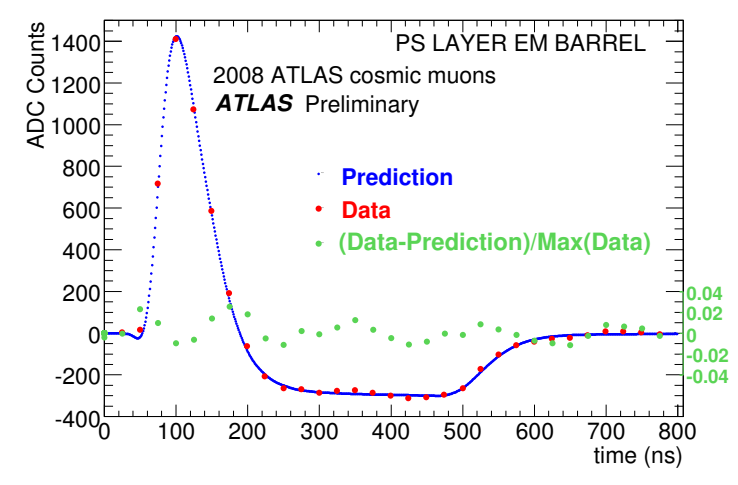

(a)

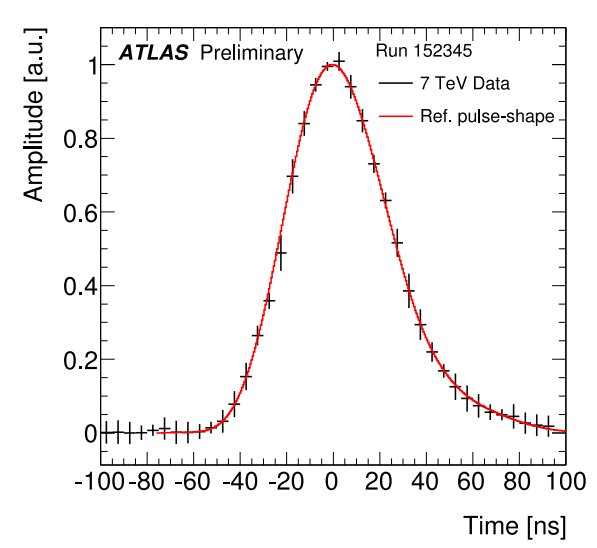

(b)

Figure 2.8.:Figure (a): sample pulse shape from cosmic ray data in the first layer of the LAr calorimeter (from [67]). Figure (b): sample pulse shape from the Tile calorimeter from a collision run, with the expected pulse shape used for reconstruction overlaid in red (from $[68]$ ).

The systematic uncertainty on the electron energy scale after this calibration procedure amounts to $3 \%$. The main sources of uncertainties are:

- The difference in the electromagnetic scale between the test-beam setup and the full ATLAS detector due to the uncertainty in the liquid argon temperature in the test-beam, derived from comparison of different test-beam measurements [65,72];

- The difference in pulse reconstruction methods and calibration corrections between the test-beam and ATLAS setup [72,77];

- The time stability of the electromagnetic scale from variations in the calibration of the electronics, and in the detector and environmental conditions monitored in test-beam and with collision data [77].

The final energy scale for the electromagnetic calorimeters needs to be determined with a higher precision with respect to that obtained with the calibration outlined above. For this purpose, correction factors are derived using $Z \rightarrow e e$ events, taking advantage of the well known mass of the $\mathrm{Z}$ boson to set the absolute energy scale and intercalibrate the various regions of the detector. If the measured energy for an electron $E^{\text {meas }}$ contains a residual miscalibration factor $\alpha$ compared with the true electron energy $E^{\text {true }}$ as $E^{\text {meas }}=E^{\text {true }}(1+\alpha)$, the effect on the measured $\mathrm{Z}$ mass $M_{Z}^{\text {meas }}$ can be parameterised as: 


\begin{tabular}{|c|c|}
\hline$\eta$ range & Fractional correction factor $(\alpha)$ \\
\hline $0 \leq|\eta|<1.37$ & $-0.96 \pm 0.01 \%$ \\
\hline $1.52 \leq|\eta|<2.47$ & $1.89 \pm 0.02 \%$ \\
\hline $2.47 \leq|\eta|<2.8$ & $-0.34 \pm 0.01 \%$ \\
\hline $2.8 \leq|\eta|<3.2$ & $-0.19 \pm 0.01 \%$ \\
\hline $3.2 \leq|\eta|<4.5$ & $5.0 \pm 0.2 \%$ \\
\hline
\end{tabular}

Table 2.1.:Fractional correction from $Z \rightarrow e e$ events applied to the electromagnetic scale of the LAr calorimeters.

$$
M_{Z}^{\text {meas }}=M_{Z}^{\text {true }}\left(1+\frac{\alpha_{i}+\alpha_{j}}{2}\right)
$$

where $\mathrm{i}$ and $\mathrm{j}$ label the electrons in the two pseudorapidity regions.

The $\alpha$ miscalibration coefficients can be derived for different calorimeter regions minimising an unbinned likelihood:

$$
-\ln L_{\text {tot }}=\sum_{k=1}^{N \text { events }}-\ln L_{i j}\left(\frac{M_{k}^{Z}}{1+\frac{\alpha_{i}+\alpha_{j}}{2}}\right)
$$

where $L_{i j}$ is a probability density function that quantifies the compatibility of the event with the $\mathrm{Z}$ lineshape based on the Breit-Wigner distribution. It has been obtained from simulated events to take into account all theoretical and experimental effects that can influence the ideal $\mathrm{Z}$ mass distribution.

Preliminary scale factors on the electromagnetic scale derived from $Z \rightarrow e e$ events were applied to the full 2010 dataset used in this thesis. These were derived using roughly 10000 events where both electrons were found in the central region $(|\eta|<2.47$, excluding the crack region) and 3100 events where one central and one forward electron $(2.47 \leq|\eta|<4.9)$ were found. The available statistics only allowed to obtain preliminary scale factors for five pseudorapidity regions, listed in Table 2.1. The scale factors are applied to all cells in the $\eta$ region considered, by scaling the cell's measured energy by $(1+\alpha)$.

The residual uncertainty on the LAr electromagnetic scale amounts to $1.5 \%$ for the electromagnetic energy deposited in the LAr calorimeters and 5\% for the energy deposited in the presampler. This accounts for the systematic uncertainties in the $Z \rightarrow e e$ analysis (that are 
detailed in the full 2010 analysis [78]) and differences in the electron shower shape between data and simulation.

\subsubsection{Hadronic calorimeters}

The hadronic calorimeters make use of plastic scintillator tiles and steel for the barrel and extended barrels (covering $0<|\eta|<0.8$ and $0.8<|\eta|<1.7$, respectively, and they are called Tile calorimeter in the following. The $3 \mathrm{~mm}$ tiles are placed perpendicular to the colliding beams, as shown in Figure 2.9. Particles interact with the active medium producing scintillation light proportional to the deposited energy, which is in turn collected using wavelength-shifting fibers. Readout cells are built by grouping fibres together in projective towers in $\eta$, and the scintillation light is collected by photomultiplier tubes at each end of the tiles. The Tile calorimeter consists of three layers in both barrel and extended barrel. The cell granularity varies according to pseudorapidity and distance from the interaction point: the granularity is $\Delta \eta \times \Delta \phi=0.1 \times 0.1$ in the first two layers and $0.1 \times 0.2$ in the third layer.

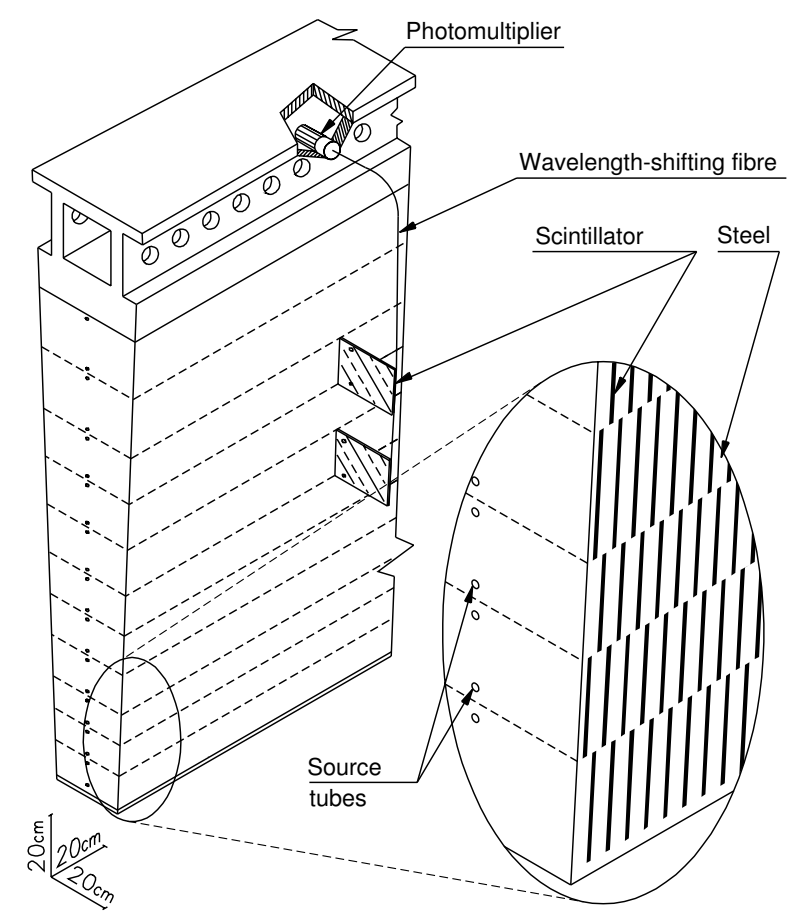

Figure 2.9.:Sketch of the structure of a module of the Tile calorimeter, showing the placement of the tiles and of the readout components. Figure from [61]. 


\section{Tile calorimeter calibration and uncertainty on the EM energy scale}

The measurement of scintillation light by the photomultipliers produces a pulse with a $50 \mathrm{~ns}$ width with amplitude proportional to the energy deposited in the cell, as shown in Figure 2.8 (b). The pulse shape is sampled 7 times and sent to the read out electronics in case the event is selected by the trigger system. An optimal filtering algorithm is used to extract the amplitude, timing and pedestal from the pulse shape [79]. The ADC counts that are the output of the photomultipliers are converted into an energy measurement using correction constants derived from test beam measurements $[65,71-73,80]$ and propagated to the full ATLAS setup using a charge injection calibration system [79]. The absolute calorimeter response to energy deposited via electromagnetic processes has also been validated in the hadronic calorimeters using muons, both from test-beams [80] and produced by cosmic-rays in-situ [81].

The uncertainty on the electromagnetic scale is estimated considering the ratio of the electromagnetic scale response of minimum ionizing low-energy muons in test-beam data and in Monte Carlo and comparing it with the same ratio between data and simulation of cosmic ray muons in the full ATLAS setup, as outlined in Reference [82]. No significant shift in the response between test-beam data and data taken with the full ATLAS detector has been observed. A $3 \%$ uncertainty on the measurement of the response ratio (due to the analysis method, the discrepancies in the behaviour of data and Monte-Carlo as a function of the muon momentum in cosmic data and the different conditions between the test-beam and ATLAS setups) has been given as the estimated uncertainty on the hadronic calorimeter EM scale [83, 84].

\section{Hadronic endcap (HEC) and forward (FCal) calorimeters}

The larger particle flux and the high energies reached in the forward region leads to the design of a calorimeter system that is able to fully contain hadronic showers and is radiation hard, at a limited cost. Liquid argon is employed as active material in the endcap and forward ATLAS calorimeters.

In the hadronic endcaps $(1.5<|\eta|<3.2$ placed at $2.03 \mathrm{~m}$ from the interaction point, called $H E C), 8.5 \mathrm{~mm}$ active gaps are sandwiched between copper plates. Each hadronic endcap consistes of two wheels, with different widths for the copper plates (25 and $50 \mathrm{~mm}$ for inner and outer wheel respectively). Each active gap is split in four drift spaces of about $1.8 \mathrm{~mm}$ by the presence of three electrodes, avoiding ion build-up and allowing a lower HV configuration with respect to a single electrode configuration. Readout cells are pseudo-projective in $\eta$ and projective in $\phi$. 
The forward calorimeter (FCal) is a liquid argon and tungsten/copper detector placed 4.7 $m$ away from the interaction point on each side, and extends the ATLAS calorimeter system up to $|\eta|<4$.9. In order to reduce the neutron flux, the front face of the FCal is placed 1.2 $\mathrm{m}$ away from the EM calorimeter front face: to allow for shower containment, the absorber must be dense. In addition, the gaps must be narrow to avoid ion build-up and have a fast readout time. To fulfill these design requirements, tungsten and copper are chosen as passive material, in a matrix that contains concentric tubular electrodes parallel to the beam pipe. The electrodes contain the liquid argon in gaps that can be as small as $270 \mu \mathrm{m}$.

The pulse shape, signal reconstruction and calibration for the endcap and forward calorimeters are similar to those of the LAr electromagnetic calorimeters, described in Section 2.5.1.

\subsection{Simulation of particle interactions in the ATLAS detector}

The GEANT4 software toolkit [85] within the ATLAS simulation framework [86] propagates the particles generated as explained in 1.4.2 through the ATLAS detector and simulates their interactions with the detector material. The energy deposited by particles in the active detector material is converted into detector signals with the same format as the ATLAS detector read-out. The detector signals are in turn reconstructed with the same reconstruction software as used for the data [86].

For the simulation of hadronic interactions in the detector, the GEANT4 set of processes called QGSP_BERT is chosen [87]. In this set of processes, the Quark Gluon String model [88] is used for the fragmentation of the nucleus, and the Bertini cascade model [89] for the description of the interactions of hadrons in the medium of the nucleus.

The GEANT4 simulation and in particular the hadronic interaction model have been validated with test-beam measurements for the barrel [80,90-93] and the endcap [74,75,94] calorimeters. Further tests have been carried out in-situ using identified single particles from kaon and lambda decays produced in proton-proton collisions, as described in Reference [95]. Excellent agreement between simulation and data has been found for pions and protons in the range of a few hundred $\mathrm{MeV}$ to $6 \mathrm{GeV}$, while the response of anti-protons is underestimated by about $10 \%$.

Studies of the material of the inner detector upstream the calorimeters have been performed using secondary hadronic interactions $[96,97]$. The ATLAS detector geometry used in the 
simulation of the nominal sample reflects the geometry of the detector ${ }^{3}$ as best known at the time of the Autumn 2010 data reprocessing that was used for this thesis.

\subsection{Software, computing and data analysis}

An efficient distributed computing system is required to collect, process and store the data recorded by the ATLAS detector. Monte Carlo simulations that mirror data as mentioned in the previous section need to be produced at regular intervals, using information from the recent data taking to improve the description of the physics processes and of the detector geometry and conditions. The software employed in ATLAS is called ATHENA, and it is a $\mathrm{C}++$ framework based on the GAUDI project [98].

\subsection{Data used in this thesis}

The data used for the inclusive jet cross section measurement presented in this thesis comprises the full 2010 ATLAS dataset (up to November 2010), corresponding to a total integrated luminosity of $37.3 \pm 1.2) \mathrm{pb}^{-1}$.

The data taken by the ATLAS detector is divided in data periods, runs and luminosity blocks. A luminosity block corresponds to the data accumulated in a small period of time (2-3 minutes of data taking), and a run is a collection of luminosity blocks corresponding to continuous data taking. The division of a run in smaller portions allows the exclusion of single problematic data taking periods with minimal impact on the overall data taking efficiency. Data periods are collections of runs that have similar overall conditions in terms of detector and trigger conditions, and are named from A to I for the data considered in this thesis.

\subsection{Trigger system}

The data rate delivered by the LHC (up to $1 \mathrm{GHz}$ in nominal conditions) largely exceeds the current capabilities for recording events offline, both in terms of recording speed and storage space. It is therefore necessary to have a system (called trigger) that selects the interesting collision events based on the presence of high transverse momentum objects (muons, electrons, photons, jets and tau leptons). The trigger system needs to reject the background without biasing the selection of the rare signals.

\footnotetext{
${ }^{3}$ The geometry tag is ATLAS-GEO-16-11-00.
} 
The trigger system is subdivided in three levels: L1, L2 and the Event Filter. The first selection is made using coarse detector information from the calorimeter, muon and forward (MBTS) subsystems in the first level trigger. The logic for the L1 trigger is mostly hardwired in the readout electronics, given that the decision needs to be made in less than $2.5 \mu \mathrm{s}$.

Since the rate for certain signatures (e.g. low $p_{T}$ QCD jets) would saturate the limited bandwidth that needs to be shared by all triggers, some triggers are pre-scaled. This means that only a fraction of the events accepted are effectively passed onto the next level, using a random reset of the passed trigger and allowing the event to fail the requirements. A prescale of 1 means that all events selected by the trigger are accepted, while larger prescales mean that only a fraction $1 /$ prescale is accepted. The trigger menu is chosen taking into account the current LHC luminosity and the physics program for each data taking period.

The events that are accepted by the L1 trigger are passed on to the L2 trigger, which further unpacks the information passed from the L1 trigger and increases the level of detail available for the decision. Both the L2 and the Event Filter are part of the software trigger subsystem called High Level Trigger (HLT). The L2 trigger has an average latency of 40ms and reduces the rate to $2.5 \mathrm{kHz}$. If the event is accepted, it is passed to the Event Filter which runs a physics reconstruction close to the offline software in about 4 seconds/event and selects events up to a rate of $200 \mathrm{~Hz}$.

The trigger systems used in the measurement described in this thesis are the Minimum Bias Trigger Scintillators (MBTS) to select minimum bias collision events in the first data periods (with a low data rate) and the jet ${ }^{4}$ triggers in the calorimeters.

The Minimum Bias Trigger Scintillators are described in more detail in $[99,100]$. They consist of plastic scintillator counters placed at $3.5 \mathrm{~m}$ away from each side of the interaction point, covering the pseudorapidity range of $2.09 \leq|\eta|<3.84$. The MBTS provide the least biased trigger configuration to select collision events by selecting events where either one or both the counters to have detected the passage of particles. They are used both to select events in the very first data taking periods and to measure efficiencies for other triggers.

Jet triggers are used to select events the main experimental signature considered in the measurement in this thesis: jets of collimated particles at high transverse momentum. The functioning and performance of the jet triggers in the first 2010 data is described in Reference [101]. The measurement of the raw calorimeter energy (calibrated to the electromagnetic scale) in a region of $\Delta \eta \times \Delta \phi=0.2 \times 0.2$ in the calorimeter system (called trigger tower) is the baseline for

\footnotetext{
${ }^{4} \mathrm{~A}$ full description of the jet definition in ATLAS is reported in Chapter 3.
} 
the Level 1 decision. A sliding window algorithm is used to select local maxima in transverse energy, and if the measured $E_{T}$ is above the trigger threshold the event is retained and the raw data within a Region Of Interest (ROI) is passed to the Level 2 trigger. The Level 2 jet trigger reconstructs a jet by drawing a cone of radius $\mathrm{R}=\sqrt{\Delta \eta \times \Delta \phi}=0.7$ and centered on the ROI, including the energy of all the trigger towers within the cone and moving iteratively (up to three times) the energy-weighted barycentre as to include the maximum local energy deposition.

The jet trigger is divided in two independent subsystems: the central jet trigger $(|\eta|<3.2)$ and the forward jet trigger $(3.2 \leq|\eta|<4.9)$. 


\section{Chapter 3.}

\section{Jet reconstruction in ATLAS}

Confined quarks and gluons from the proton-proton scattering manifest themselves as groups of collimated particles in the final state, as described in Section 1.3. The particles are clustered into physically measurable objects at hadron colliders: these objects are called jets.

The concept of a jet is not meaningful without a full specification of its definition. Defining a jet requires a procedure to associate either particles or energy deposits to a single jet (jet algorithm) and a recombination scheme that specifies how to combine the four-momenta of the jet constituents. The jet definition, the main jet algorithms and the inputs to jets in the ATLAS detector are described in Section 3.1.

Jets measured in the ATLAS detector need to be fully reconstructed in order to reconnect the the energy measured in the calorimeter to the kinematics of the particles or partons that generated the jets. First, the calorimeter energy deposits need to be associated to a single jet with a jet finding algorithm. Subsequently, a calibration procedure is needed to translate the signals read out from the calorimeters into a measurement of the energy of the particles forming the jet. Section 3.2 motivates the jet calibration procedures and and outlines the calibration scheme used in the 2010 ATLAS dataset. Techniques undergoing commissioning are briefly mentioned.

\subsection{Jet algorithms and inputs to jet reconstruction}

Jets are connected to the underlying hard parton process. Since partons are not physically observable objects, there is no unique jet definition. Nevertheless, in order to provide common grounds of interpretation for jet physics between theory and experiments, a common definition needs to be used to uniquely map a set of four-momenta (partons, particles or calorimeter objects) into a jet. A jet definition, as suggested in the 2007 Les Houches accord [102], comprises: 
- a jet algorithm that defines how to group the four-momenta into jets;

- the full specification of the jet algorithm parameters;

- the recombination scheme: a set of a rules for obtaining the four-momentum of a jet from its constituents.

The specification of the truth level (Monte Carlo) particles that are inputs to jets used for the theory/experiment comparison is also needed, for example in the case where where detector effects are corrected by an unfolding technique as in the inclusive jet measurement presented in this thesis (Chapter 6).

The following sections are dedicated to an overview of the properties required in a jet algorithm (Section 3.1.1) and to the details of the jet definition used in ATLAS (Sections 3.1.2 and 3.1.3).

\subsubsection{Theoretical requirements for a jet algorithm}

A jet algorithm is required to be well behaved when confronted with possible QCD divergences, such as infrared and collinear emissions, detailed in Chapter 1. Both infrared and collinear emissions manifest themselves as soft particles, and the jet algorithm needs to be resilient to these by reconstructing the same set of jets regardless of their presence or absence. Theoretically well behaved jets give finite perturbative results at all orders, and allow meaningful comparisons of data with the most recent theoretical predictions.

A theoretically well behaved jet algorithm needs to be infrared and collinear safe: The presence or absence of additional infinitely soft particles radiated by the primary partons should not modify the result of jet finding (e.g. the number of jets). Furthermore, jets should not be sensitive to particles radiated at very small angle with respect to the original parton. An example of the behaviour of an algorithm that does not behave well in presence of soft QCD radiation is shown in Figure 3.1.

In addition to infrared and collinear safety, a jet algorithm should reproduce the same physics in the final state regardless of the input type: jets can be reconstructed from partons, particles and calorimeter objects.

In order to be successfully used in a high energy physics experiment, a jet algorithm needs to have a computationally fast and fully specified implementation. Many modern jet algorithms are implemented in the FastJet software $[103,104]$ either natively or as plug-ins. The jet reconstruction software in ATHENA employs the FastJet classes for jet finding. 


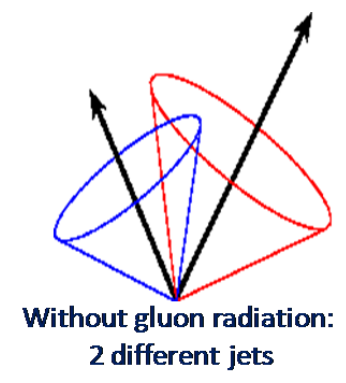

(a)

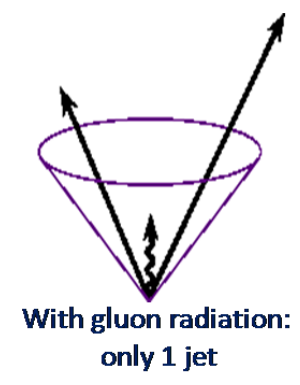

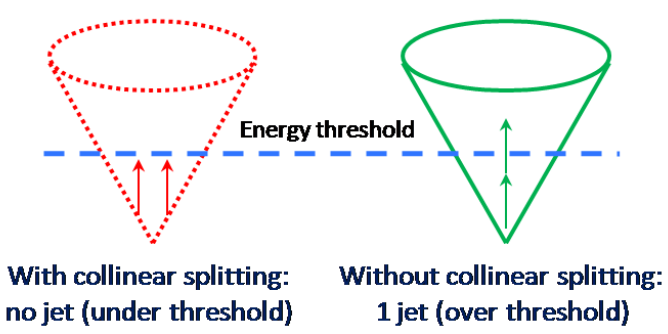

(b)

Figure 3.1.:Example behaviour of an infrared unsafe algorithm (a) and of a collinear unsafe algorithm (b).

\subsubsection{Jet definition in ATLAS}

Jet algorithms can be divided in two classes: cone algorithms and successive recombination algorithms.

Cone algorithms define jets based on the dominant direction of energy flow. The ATLAS Cone algorithm, employed before data taking, starts from jet inputs with energy above a certain threshold (seeds) and sums the four-momenta of all particles in a surrounding cone in $\eta, \phi$ space with a radius $R$ to obtain the initial proto-jet direction. A cone of radius $R$ is then redrawn starting from the proto-jet direction and four-momenta summed to obtain a new jet direction. The procedure is iterated until the jet direction is stable (does not change significantly in successive iterations). A split-merge step is performed to assign overlapping constituents to the closest jet or merge two overlapping jets depending on the fraction of constituents shared by the two jets.

Successive recombination algorithms iteratively merge pairs of objects according to a definition of distance that typically involves the physical distance between the objects and their transverse momentum, and in certain cases effectively undoing the QCD parton shower. The jet algorithm employed in ATLAS data taking and for the measurement described in this thesis is the anti- $k_{t}$ algorithm. This jet algorithm is based on the comparison for all possible pairs of objects between the distances $d_{i, j}=\min \left(p_{T, i}^{-2}, p_{T, j}^{-2}\right) \frac{\Delta R}{R}$ and $d_{i, \text { Beam }}=p_{T, i}^{-2}$, where $p_{T}$ is the transverse momentum of the particle considered, $\Delta R$ is the spatial distance of the two objects in $\eta-\phi$ space and $R$ is a parameter of the algorithm which can be considered as a weight to the spatial distance of the two objects - the larger $\mathrm{R}$, the wider the jets. In the following, $R$ will be referred to as the jet algorithm distance parameter. If the minimum between all $d_{i, j}$ and $d_{i, \text { Beam }}$ is a $d_{i, j}$, objects $i$ and $j$ will be recombined, otherwise object $i$ is considered a jet and not considered in the next iteration. With this algorithm, soft radiation is effectively clustered around the harder core of 
the process. Anti- $k_{t}$ jets have a regular, conical shape, experimentally desirable since it allows for a well defined jet area that can be used for e.g. pile-up subtraction [105].

The ATLAS Cone algorithm is not theoretically well behaved due to the presence of a seed with which to start the jet finding and to the split/merge step: soft particles originating from the hard parton might influence whether the parton passes the threshold to form a proto-jet, and influence whether the final jet is split into two separate jets. The anti- $k_{t}$ algorithm described below does not suffer from any theoretical problems. This, and the better performance before and after calibration [106] (including a better trigger matching performance), are the reasons why the ATLAS collaboration adopted the anti- $k_{t}$ algorithm for data taking. Two different distance parameters of $\mathrm{R}=0.4$ and $\mathrm{R}=0.6$ are employed for the measurements in this thesis, as detailed in Chapter 6, as jets with different areas will be impacted differently by non perturbative effects such as hadronisation and underlying event.

The final four-momentum of the jet is obtained from summing the four-momenta of its constituents in the four-vector recombination scheme. This scheme conserves energy and momentum, and allows a meaningful definition for the jet mass.

\subsubsection{Inputs to jet reconstruction}

Jets in ATLAS are reconstructed starting from different physics objects [107,108]. The most widely used inputs to jet finding are topological clusters, or topoclusters [69], groups of calorimeter cells clustered into three-dimensional energy deposits exploiting the longitudinal and transverse calorimeter segmentation. Calorimeter cells are included in topoclusters using a noise suppression scheme. Jets built from topological clusters will be referred to as topocluster jets in the following. Jets can also be built using groups of geometrically delimited groups of calorimeter cells (towers), that can also take advantage of the noise suppression scheme; these jets will be called tower jets in the following. Both topoclusters and towers are reconstructed as massless pseudo-particles, and are described in more detail below. Other inputs to jet reconstruction can be reconstructed tracks (track jets) or stable ${ }^{1}$ particles generated by the Monte Carlo event generator (Monte Carlo truth jets). Truth jets used for the calibration and performance studies do not include muon and neutrinos, while truth jets used for unfolding detector effects in the inclusive jet cross section measurement do.

\footnotetext{
${ }^{1} \mathrm{~A}$ stable particle is defined to have a lifetime longer than $10 \mathrm{ps}$.
} 


\section{Topological clusters}

Topological clusters are groups of calorimeter cells that are designed to follow the shower development taking advantage of the fine segmentation of the ATLAS calorimeters. The topocluster formation algorithm starts from a seed cell, whose signal-to-noise ratio (estimated as the energy deposited in the calorimeter cell over the RMS of the energy distribution measured in randomly triggered events) is above a threshold of 4 . Cells neighboring the seed that have a signal-to-noise ratio of at least 2 are included iteratively, and finally all neighboring cells are added to the topocluster. The topoclustering algorithm also includes a splitting step: all cells in a cluster are searched for local maxima in terms of energy content, and the local maxima are then used as seeds for a new iteration of topological clustering, which will split the original cluster in more topoclusters. A topocluster is defined to have an energy equal to the energy sum of all the included cells, zero mass and a reconstructed direction as that of a unit vector originating from the centre of the ATLAS coordinate system pointing to the energy-weighted topocluster barycentre. The energy scale of topoclusters can also be calibrated starting from the baseline energy scale measured by the calorimeters, as explained in section 3.2.2.

\section{Calorimeter towers}

Towers are defined as groups of calorimeter cells that are delimited by a projective fixed two-dimensional grid in pseudorapidity and azimuthal angle. The chosen grid element size is $\eta \times \phi=0.1 \times 0.1$; this choice divides the calorimeter into approximately 6400 geometrical towers of fixed size in detector $(\eta, \phi)$ space ${ }^{2}$. The energy of a tower is given by the sum of the energies of the single calorimeter cells as measured by the calorimeters. This definition allows a fixed delimitation of the energy that contributes to a jet, and therefore allows for a clear definition of the jet area to be employed when subtracting additional baseline energy that does not belong to the jet (e.g. due to multiple proton-proton interactions within the same bunch crossing) is performed.

Cells contributing to a tower can have a positive or a negative signal; the latter happens in the case of noisy cells or cells integrating energy from more than one proton-proton interaction. Summing all contributions indiscriminately allows for a natural average noise suppression, but it also leads to negative towers in the case of large negative cell energies. Since negative energy constituents have no clear meaning in four-vector algebra, unphysical results can be obtained when reconstructing jets. For this reason, jets formed by towers with no explicit noise

\footnotetext{
${ }^{2}$ For non-projective calorimeter cells (e.g. in the FCal) or for cells larger than the tower grid element size, the energy contribution of the cell to the tower is weighted by the ratio of the tower bin area over the cell area in $\eta, \phi$.
} 


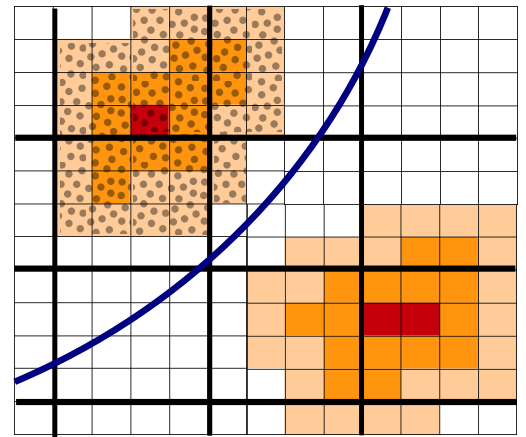

$\Delta \eta=0.1$

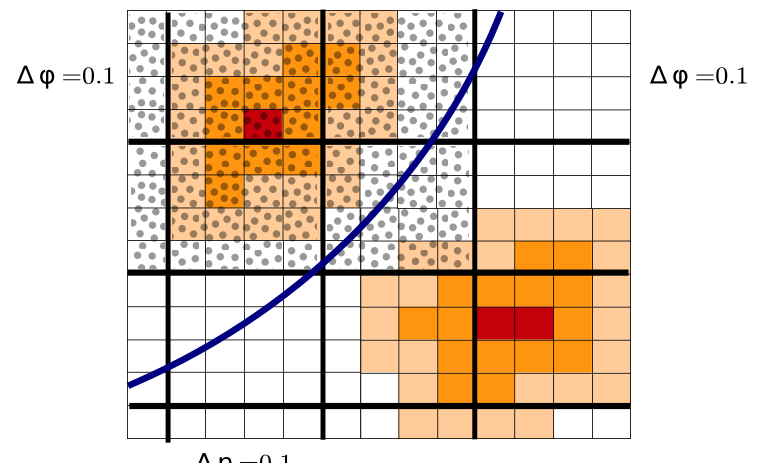

(a)

(b)

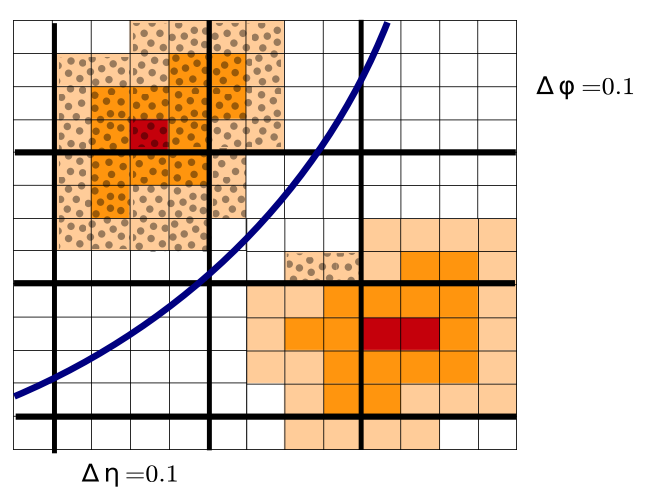

(c)

Figure 3.2.:Schematic representation of the three different types of jet inputs. Topoclusters are shown in Figure (a) with shaded areas corresponding to energy deposits above the noise-suppression thresholds, towers without noise suppression in Figure (b) and noise suppressed towers in Figure (c). The cells considered for jet finding are dotted, while the dark blue line shows a simplified edge of a jet.

suppression are only used for specific studies, and the default tower definition only includes cells that are contained in topoclusters, automatically discarding negative energy constituents. The noise-suppressed towers are called topological towers or topotowers.

Figure 3.2 shows drawings of the different types of jet inputs: topoclusters (with the different signal/noise contributions in shades of red), towers and topotowers. Topoclusters have been drawn as composed by the shaded cells passing the noise suppression threshold. Dotted cells are the ones that are effectively input to jet finding.

\subsection{Jet energy scale calibration in ATLAS}

In non-compensating calorimeters such as the ones in the ATLAS experiment, the response to hadrons is lower than the response to particles interacting electromagnetically. This Section 
describes the calibration schemes that have been developed in ATLAS to restore the correct hadronic energy scale starting from the electromagnetic scale. The conversion of the raw calorimeter signal into a EM-scale calibrated energy measurement has been described in Sections 2.5.1 and 2.5.2. A correction for calorimeter non-compensation is needed to restore the final hadronic energy scale (jet energy scale), together with further corrections to take into account effects related to the detector, such as:

1. energy losses in inactive regions of the detector (dead material),

2. energy deposits from particles not contained in the calorimeter (leakage),

3. energy deposits of particles not included in the reconstructed jet because e.g. they have been deviated by the magnetic field (out of cone),

4. signal losses in calorimeter clustering and jet reconstruction.

Presently, the default ATLAS calibration scheme applies calibration corrections as a function of the jet energy and pseudorapidity to jets reconstructed at the electromagnetic scale. The hadronic jet energy scale is on average restored using calibration constants derived from the comparison of the reconstructed jet kinematics to that of the corresponding truth level jet in Monte Carlo studies. The additional energy due to multiple proton-proton interactions within the same bunch crossings (pile-up) is corrected for before the hadronic energy scale is restored using data-derived factors, so that the derivation of the jet energy scale calibration constants is factorised and does not depend on the number of additional interactions measured. This calibration scheme (called EM+JES) has been validated with in-situ techniques. It also allows a direct evaluation of the systematic uncertainty, being therefore suitable for the very first physics analyses.

The baseline (nominal) Monte Carlo sample used to derive the jet energy scale and to estimate the sources of its systematic uncertainty is composed of inclusive QCD jet events generated with the PYTHIA event generator tuned to the first ATLAS data [46] and passed through the full ATLAS detector simulation. The nominal sample has been described in more detail in Section 1.4.2. The reliability of the application of this Monte Carlo-based JES calibration to data requires that reconstructed jets in this Monte Carlo sample are in good agreement with jets in data. The PythiA Monte Carlo simulation has been shown to describe data reasonably well, as shown in Figure 1.7. 


\subsubsection{EM+JES calibration scheme}

The EM+JES calibration scheme is applied to both topocluster and tower jets after jet finding has been performed at the electromagnetic scale, as a series of subsequent steps shown in figure 3.3 and detailed in the following paragraphs.

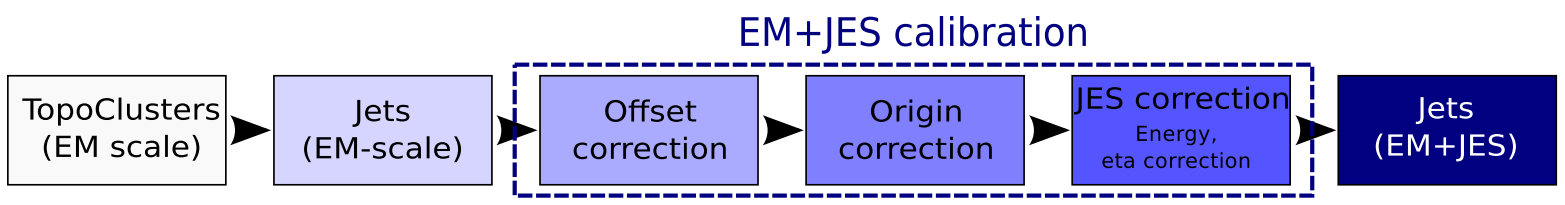

Figure 3.3.:Schematic representation of the jet reconstruction procedure for EM+JES jets.

\section{Pile-up correction}

The energy of jets can include energy that does not come from the interaction of interest, but is instead produced by multiple proton-proton interactions within the same bunch crossing. A correction is derived from minimum bias data as a function of number of reconstructed primary vertices $N_{\mathrm{PV}}{ }^{3}$ and jet pseudorapidity $\eta$, and takes into account the average additional energy deposited in a fixed grid of $0.1 \times 0.1$ in the $(\eta, \phi)$-plane (non-noise suppressed calorimeter towers). To derive the correction, the average additional energy is then multiplied by the number of towers in jets built from towers, or the average number of towers in the case of topocluster jets, as a function of jet pseudorapidity. This correction is applied at the electromagnetic scale as the first step of the calibration scheme. Further details on the pile-up offset correction can be found in in Ref. [57].

\section{Jet origin correction}

Calorimeter jets are reconstructed using the geometrical centre of the ATLAS detector as reference to calculate the direction of jets and their constituents (see Section 3.1.3). The direction of each topocluster or tower included in a jet is corrected to point back to the primary vertex with the highest associated sum of track transverse momenta squared $\left(\sum p_{T, \text { track }}^{2}\right)$ in the event. The kinematics of each jet constituent is recalculated using the vector from the primary vertex to the topocluster or tower centroid as its direction. The raw jet four-momentum is thereafter redefined as the vector sum of the constituent four-momenta, and its pseudorapidity is defined $\eta_{\text {origin }}$ in contrast to the raw detector pseudorapidity measured in the calorimeters $\eta_{\text {det }}$. The origin correction improves the angular resolution, while the jet energy is unaffected.

\footnotetext{
${ }^{3}$ Vertices are counted using a criterion of having at least 5 associated tracks.
} 


\section{Final JES correction}

In the final step of the EM+JES scheme, calibration constants derived from the comparison of the simulated calorimeter jet kinematics to the kinematics of the corresponding Monte Carlo truth jet are applied. Since pile-up effects have already been corrected for, the Monte Carlo sample used to derive the calibration constant does not include multiple proton-proton interactions within the same bunch crossing.

Only isolated jets are used for the calibration: an isolated jet is defined as a jet that has no other calorimeter (truth) jet with EM-scale (truth) $p_{T}>7 \mathrm{GeV}$ within $\Delta R=2.5 R$, where $R$ is the distance parameter of the jet algorithm. The choice of using a specific Monte Carlo sample with an isolation requirement leads to the calibration to be tailored to the specific jet flavour composition (e.g. quark jets vs gluon jets) and topology used to derive the calibration constants. Effects related to close-by jets and flavour composition need to be taken into account at the analysis level and are discussed in Section 5.6. Only calorimeter jets that are geometrically matched to truth jets within a cone of $\Delta R=\sqrt{(\Delta \phi)^{2}+(\Delta \eta)^{2}}=0.3$ around the jet axis are used for the calibration.

For each pair of matched calorimeter/truth jet, the energy response $\mathcal{R}$ is defined as the ratio of the jet energy measured in the calorimeters at a given scale $E_{\text {calo }}$ and the energy of the matched truth jet $E_{\text {truth }}$ :

$$
\mathcal{R}=E_{\text {calo }} / E_{\text {truth }}
$$

To derive the JES correction, the first step is to calculate the jet energy response at the electromagnetic scale $\mathcal{R}_{\mathrm{EM}}$ using the calorimeter energy as measured by the calorimeter $E_{\text {calo }}^{\mathrm{EM}}$. For each $\left(E_{\text {truth }}, \eta_{\text {det }}\right)$-bin considered, two histograms for $\mathcal{R}_{\mathrm{EM}}$ and $E_{\text {calo }}^{\mathrm{EM}}$ are filled. For each bin of $\eta_{\text {det }}$, the mean of a Gaussian fit to the $\mathcal{R}_{\mathrm{EM}}$ distribution defines the average EM-scale energy response $\langle\mathcal{R}\rangle$, as a function of the average $E_{\text {calo }}^{\mathrm{EM}}$ corresponding to the $E_{\text {truth }}$ bin considered. $\left\langle\mathcal{R}\left(E_{\text {calo }}^{\mathrm{EM}}\right)\right\rangle$ is then fitted for each $\eta_{\text {det }}$ bin $k$ with an empirical function parameterised as:

$$
\mathcal{F}_{\text {calib }, k}\left(E_{\text {calo }}^{\mathrm{EM}}\right)=\sum_{i=0}^{N_{\max }} a_{i}\left(\ln E_{\text {calo }}^{\mathrm{EM}}\right)^{i},
$$


where $a_{i}$ are free parameters, and $N_{\max }$ is chosen between 1 and 6 depending on the goodness of the fit. In the case of jets calibrated starting from the electromagnetic scale, the fit is constrained to pass through three points lying on a curve that follows an approximate expression by Groom et al. $[109,110]$. This helps convergence at very high energies where the Monte Carlo statistics is limited. Groom's function exploits the consideration that at high energies bremsstrahlung and $\pi_{0} \rightarrow \gamma \gamma$ dominate the hadronic shower, and therefore the electromagnetic fraction (that does not need an additional correction due to non-compensation) increases with energy.

The final jet energy scale correction that relates the measured calorimeter jet energy scale to the hadronic scale for each separate $\eta_{\text {det }}$-bin is then defined as $1 / \mathcal{F}_{\text {calib,k }}\left(E_{\text {calo }}^{\mathrm{EM}}\right)$ in the following:

$$
E_{\text {calo }}^{\mathrm{EM}+\mathrm{JES}}=\frac{E_{\text {calo }}^{\mathrm{EM}}}{\left.\mathcal{F}_{\text {calib }}\left(E_{\text {calo }}^{\mathrm{EM}}\right)\right|_{\eta_{\text {det }}}},
$$

The average jet energy scale correction $\left\langle 1 / \mathcal{F}_{\text {calib, },}\left(E_{\text {calo }}^{\mathrm{EM}}\right)\right\rangle$ is shown as a function of calibrated jet transverse momentum $p_{\mathrm{T}}^{\text {jet }}$ for three jet $\eta$-intervals in Figure 3.4. The value of the correction factor ranges from about 2.1 at low jet transverse momentum to less than 1.2 for high energy jets in the most forward region. The correction is only shown over the accessible kinematic range, i.e. values for jets above the kinematic limit are not shown. This is also the case for the following figures in this thesis. The calorimeter jet response at the electromagnetic scale $\mathcal{R}_{\mathrm{EM}}$ is shown for various energy- and $\eta_{\text {det }}$-bins in Figure 3.5.

After the jet origin and energy corrections, a small $\eta$-dependent correction is applied to remove a bias in the reconstructed $\eta$ of jets that occurs when the jet falls into a poorly instrumented region of the calorimeter. In these regions topoclusters are reconstructed with a lower electromagnetic scale energy with respect to better instrumented regions (see Figure 3.5). When clustered with a jet algorithm, the energy of each topocluster is used as a weight in the calculation of the jet direction: the jet direction will therefore be biased towards the better instrumented calorimeter regions. The size of this bias is illustrated as a function of uncorrected detector pseudorapidity $\left|\eta_{\text {det }}\right|$ and EM+JES calibrated jet energy in Figure 3.6.

The $\eta$-correction is derived as the average $\Delta \eta=\eta_{\text {truth }}-\eta_{\text {origin }}$ in $\left(E_{\text {truth }}, \eta_{\text {det }}\right)$-bins, and is parameterised as a function of the jet $E_{\text {calo }}^{\mathrm{EM}+\mathrm{JES}}$ and $\eta_{\text {det. }}$. It is very small $(\Delta \eta<0.01)$ for most regions of the calorimeter but larger in the transition regions (up to $\Delta \eta=0.05$ for low $p_{T}$ jets in the HEC-FCal transition region around $\eta_{\text {det }}=3.2$ ). 


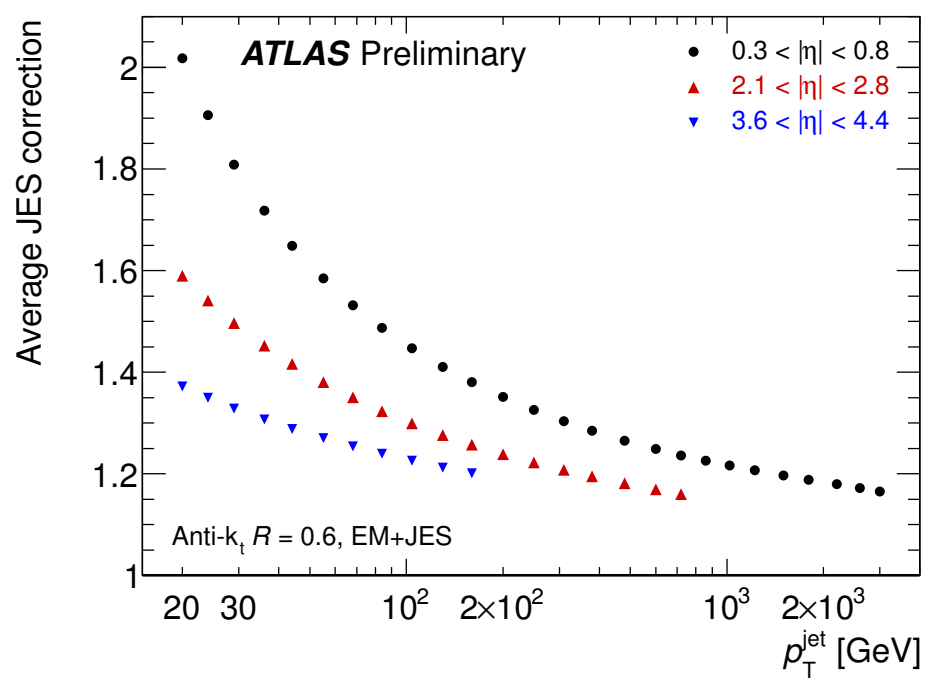

Figure 3.4.:Average jet energy scale correction as a function of calibrated jet transverse momentum in three detector pseudorapidity bins. The correction is only shown over the accessible kinematic range, i.e. values for jets above the kinematic limit are not shown. Figure from [111].

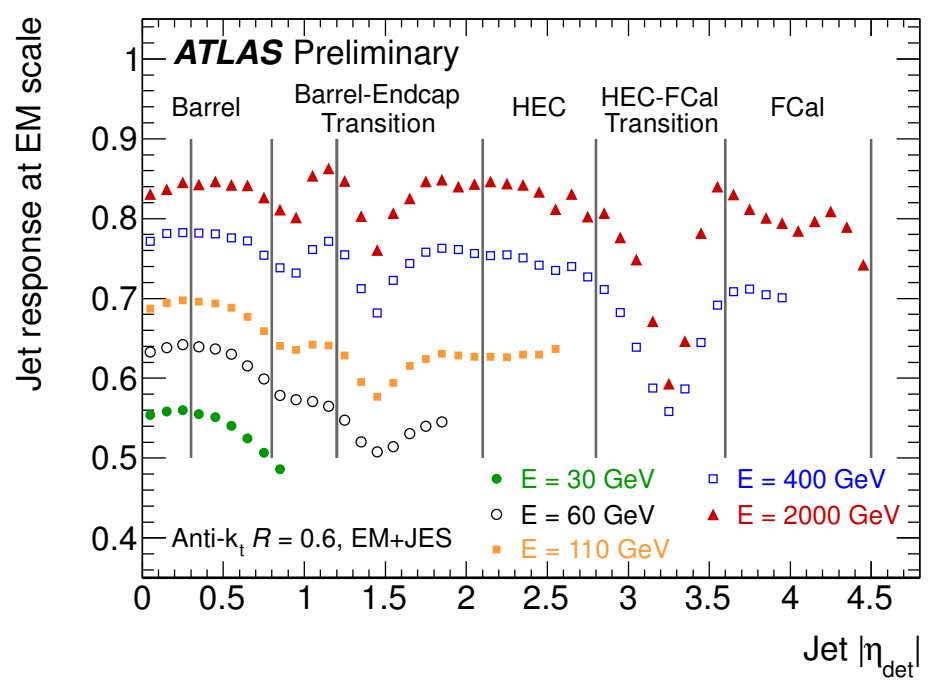

Figure 3.5.:Simulated jet energy response at the electromagnetic scale as a function of detector pseudorapidity $\eta_{\text {det }}$ and for different EM+JES calibrated jet energies $E_{\text {calo }}^{\mathrm{EM}+\mathrm{JES}}$ (called $E$ in the plot). The inverse of the response shown in each bin is equal to the average jet energy scale correction (and therefore equal to $\mathcal{F}_{\text {calib }}$ ). Figure from [111].

\subsubsection{Other calibration schemes}

In addition to the EM+JES scheme, other calibration schemes are undergoing commissioning in ATLAS. The different characteristics of electromagnetic and hadronic showers are exploited as cluster-by-cluster and/or jet-by-jet information. This reduces some of the sources of fluctuations 


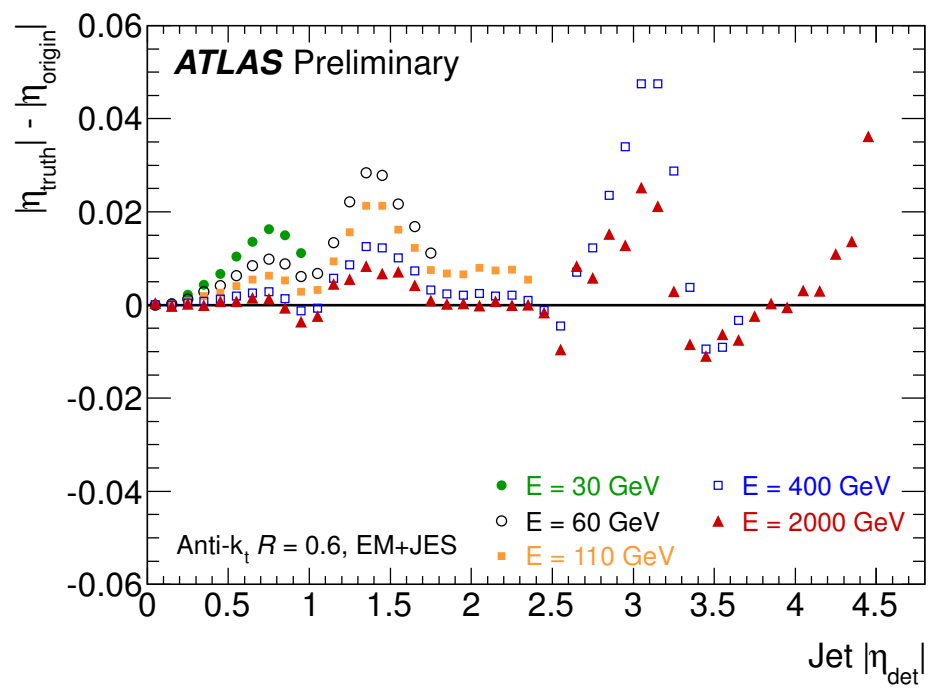

Figure 3.6.:Jet pseudorapidity bias as a function of detector pseudorapidity $\eta_{\text {det }}$ and for different EM+JES calibrated jet energies E. Figure from [111].

in the jet energy response (as described in Section 4.1) that are still present when applying an overall scale factor to calibrate jets, and thereby improves the jet energy resolution. Hadronic cascades are more extended and less dense than the electromagnetic ones. Cell energy densities and specific jet properties can be measured thanks to the high granularity of the calorimeters and used to parameterise the correction factors that compensate for the different response of hadronic and electromagnetic showers.

Hadronic calibration methods in ATLAS can be classified in two categories:

1. Global calibrations, in which the jet finding is performed on either topocluster or towers at the electromagnetic scale and then the energy of the whole jet or of the cells belonging to a jet is corrected to the final jet energy scale;

2. Local calibrations, in which the jet finding is performed on topological clusters which have already undergone a reweighting based on the properties of the cluster.

The EM+JES scheme described above, the Global Sequential Calibration [54] and Global Cell Weighting [54,112] schemes belong to the first category, while the Local Cluster Weighting scheme [113] belongs to the second. A brief description of these calibration schemes is given in the following.

Global Cell Weighting In the Global Cell Weighting scheme (GCW), jets are grouped from calorimeter objects calibrated at the electromagnetic scale, and subsequently the cells that form the calorimeter objects belonging to the jet are resummed with a correction weight 


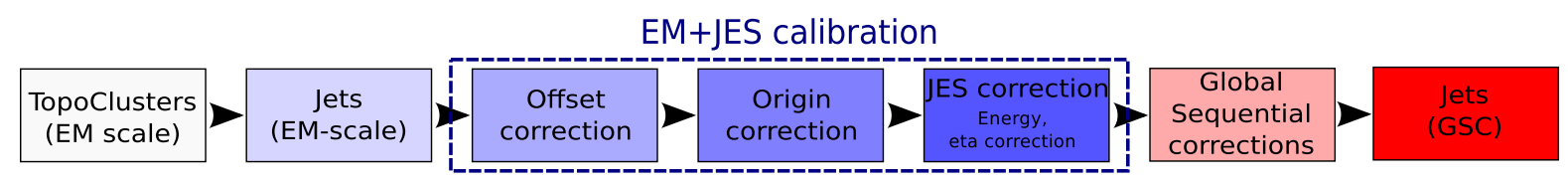

(a)

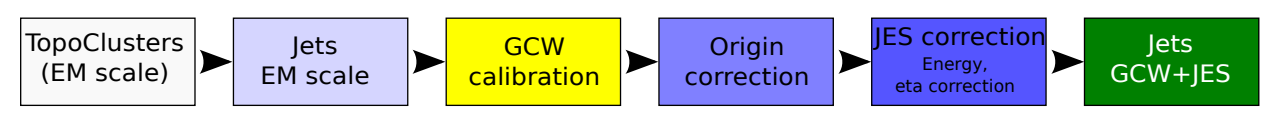

(b)

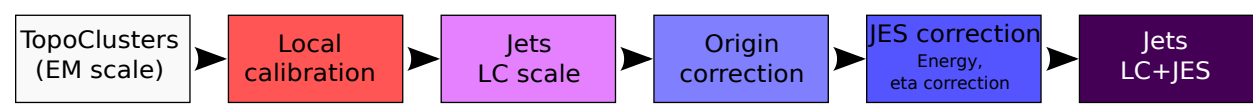

(c)

Figure 3.7.:Schematic representation of the jet reconstruction procedure for jets calibrated with the Global Sequential (a), Global Cell Weighting (b) and Local Cell Weighting (c) calibration schemes.

applied. The weights are parameterised according to the energy density and position of the cell. In order to have a reference jet from which to obtain the calibration weights, jet finding is performed on simulated particles (at the true level before entering the detector) and the reconstructed energy is compared to the true energy: the weights are then obtained via $\chi^{2}$ minimization on a simulated dijet sample with a specific jet finding algorithm.

Global Sequential Calibration The Global Sequential Calibration (GS) uses correction factors that depend on the longitudinal and transverse jet structure properties. It is applied as an additional correction on top of EM+JES calibrated jets and aims to improve the jet energy resolution without modifying the average jet response. The variables used to parameterise the correction are:

- the fraction of energy in a given layer $f_{\text {layer }}=E_{\text {layer }}^{E M} / E_{\text {jet }}^{E M}$ for one or more calorimeter layers depending on the jet pseudorapidity

- the jet width calculated as the average distance of a jet constituent to the jet direction, weighted with the energy of the constituent.

The correction is derived as a multiplicative factor to be applied to the full four-vector by inverting the jet response at the EM+JES scale as a function of each of the variables listed above. Different variables are used sequentially: jets corrected with the $\mathrm{n}-1$ th variable factor are used as input to calculate the jet response as a function of the nth variable and so forth. The dependence of the jet energy scale from the jet longitudinal and transverse 
properties is removed, therefore reducing the width of the jet energy distribution, but the average response is left unchanged.

Local Cluster Weighting In the Local Cluster Weighting calibration scheme (LC, or LCW) the energy of topological clusters is corrected first, and jet finding is then performed on the calibrated objects. The corrections are modular, and only topological clusters that are classified as hadronic from their characteristics are corrected with a weight that depends on the position and the energy density of the constituent cells. In this case the weights have been derived from simulation of single pions and verified in test beam measurements [114]. Jet level corrections are then applied after the jet finding in order to account for unclustered energy and dead material. Thanks to the cluster classification, the scaling factors applied to the electromagnetic energy deposits can be as close as possible to unity.

Methods that apply corrections directly to jets are physics dependent, since the cell weights have been obtained by comparing the reconstructed jet energy to the energy of particle jets from Monte-Carlo for a specific sample (the nominal dijet sample described in Section 1.4.2) and a specific jet finding procedure. The advantage of the Local Cell Weighting scheme is that the corrections applied to the calorimeter objects are modular and have been derived from simulation of single particle, and do not depend on the physics sample or on the jet finding algorithm. All inputs to the various jet calibration schemes in Monte Carlo simulations (e.g. longitudinal and transverse jet properties for the Global Sequential Calibration, cell energy densities for Global Cell Weighting) have been compared to measurements using the first ATLAS data, and shown to be in reasonable agreement [54]. In the case of the jet width, where data and Monte Carlo discrepancies are not fully understood, the relative Global Sequential Calibration correction is not applied.

These refined calibrations can be employed in precision analyses where jet resolution effects need to be reduced to a minimum. The Local Cluster Weighting scheme has been particularly relevant in the very first ATLAS data when calculating the sum of all energy deposited in the calorimeter $\left(\Sigma E_{T}\right)$ or the missing transverse momentum, since single calibrated topoclusters have been used before jets were fully commissioned.

\section{Additional corrections for alternative calibration schemes}

The offset correction for pile-up is only used in combination with the EM+JES and Global Sequential calibration schemes; event by event and jet by jet correction techniques to account for the average increase of the jet energy due to pile-up have been studied in Monte Carlo and are undergoing commissioning with data. 
The correction to the jet origin is commissioned for the Global Cell Weighting scheme in addition to the EM+JES and Global Sequential calibrations, and corrects the energy of each single cell belonging to the jet to point to the primary interaction vertex before the energy weighting step. Locally calibrated topoclusters included in a jet also have an origin correction applied to their direction.

Both the Local and Global Cell Weighting schemes need an additional JES correction similar to the last step of Section 3.2.1, to fully restore the jet energy scale. The final JES correction is applied to these calibrations with the following differences:

1. The starting scale for the derivation and application of the correction is the calibrated scale, not the electromagnetic scale;

2. The correction function is not forced to pass through the three points derived from a Groom distribution, since the non-compensation is partially recovered by the previous calibration step.

In the following, the Local Cluster Weighting and Global Cell Weighting after this correction has been applied will be denoted as LC+JES and GCW+JES, while the Global Sequential Calibration will be denoted as GS.

The full jet reconstruction procedure for jets calibrated with the Global Sequential Calibration, Global Cell Weighting and Local Cell Weighting schemes is pictured in Fig. 3.7. 


\section{Chapter 4.}

\section{Jet reconstruction performance}

Since jets are key ingredients for measurements at hadron colliders, the evaluation of their performance is particularly relevant for physics analyses.

The two key quantities that are input to physics analyses are the jet energy and transverse momentum response and resolution. The jet energy response and resolution are respectively connected to the mean and the width of the distribution of the energies of a jet originating from an object whose true energy is within a given (narrow) range. Jet calibration techniques such as those described in Chapter 5 are designed to restore the jet energy scale and could help to reduce the fluctuation in the reconstructed jet energy. The performance of jet reconstruction can be tested using Monte Carlo simulation by comparing reconstructed jets to truth particle jets, but it is particularly important that the performance of Monte Carlo based jet energy calibrations applied to data is tested using the data itself, using in-situ techniques.

This chapter is concerned with the methods used to quantify the performance of jet reconstruction and calibration in terms of the jet energy and $p_{T}$ response and resolution. It starts with a brief description of the jet energy resolution and its measurement in ATLAS in Section 4.1. Techniques that make use of data taken in 2010 to evaluate the jet energy scale in-situ are described in Section 4.2 (these two sections are complemented by Appendix A, where additional details on the various techniques can be found). The full evaluation of the jet energy scale uncertainty is described in the next chapter.

The various jet reconstruction steps also need to be validated before the full procedure is deployed in the software used for data taking. For the full 2010 data taking period, this has been done using Monte Carlo simulation. The tools used for this purpose (the JetPerformance package) are described in Appendix B, and the figures of merit and plots are reported in Section 4.3 


\subsection{Jet energy resolution}

The energy of a reconstructed jet can fluctuate with respect to the initial energy of the originating object for a number of reasons, related to the behaviour of partons and jets dictated by the underlying QCD theory or connected to instrumental effects. Among these are particles escaping the finite jet area or additional particles not from the hard scatter being collected within the jet, the statistical fluctuations inherent to the energy measurement in calorimeters, the inhomogeneous energy losses between active and passive material, calorimeter non uniformities and non linearities...

Quantifying the magnitude of these fluctuations in terms of the width of the jet response distribution $^{1}$ is essential in order to recover correspondence between the jet energy measured in the calorimeter and the energy of the underlying physics object. The knowledge of the jet resolution has an impact on the jet cross section measurement described in this thesis: knowing how well the Monte Carlo simulation describes jet energy resolution in the data is necessary in order to unfold all detector effects and compare the measurement to theory.

The two different methods to measure the jet energy resolution in data are employed by ATLAS are briefly described in Section 4.1.1 and more detail is given in Appendix A.1. The jet energy resolution can also be measured in Monte Carlo (Section 4.3.3), by taking the width of the jet response distribution (where the jet response is defined as in Section 3.2.1) and dividing it by its mean ${ }^{2}$. This quantity will be named truth resolution in the following.

Comparing the resolution evaluated with in-situ methods that are applied to data and Monte Carlo can give confidence that the truth resolution is a good representation of the jet resolution in data. In the following, the jet energy resolution will be replaced by the transverse momentum resolution since the jet $p_{T}$ is the most commonly employed variable in physics analysis, and the fractional resolution $\left(\frac{\sigma_{p_{T}}}{p_{T}}\right)$ will be used as benchmark quantity.

\subsubsection{In-situ determination of the jet energy resolution}

ATLAS employs two different techniques to measure the jet energy resolution using calorimeter observables: the dijet balance method and the bisector method, described in References $[115,116]$.

\footnotetext{
${ }^{1}$ Most effects mentioned here lead to a Gaussian distribution of the jet response, but some contribute to its tails. As a first iteration, most techniques used to estimate the jet energy resolution are mainly directed towards the measurement of the Gaussian width of the jet response distribution.

${ }^{2}$ It can be shown that the fractional jet $p_{T}$ resolution in Monte Carlo can be approximated by the Gaussian width of the $p_{T}$ response distribution $\mathcal{R}$ as in a given $\left(p_{T}^{\text {true }}, \eta\right)$ bin and dividing it by the mean response using the assumption of narrow $p_{T}^{\text {true }}$ bins.
} 
The two techniques rely on different assumptions and have different systematic uncertainties: two independent measurements of the jet energy resolution can be compared and they can provide separate cross-checks to the Monte Carlo simulation.

Fluctuations in the jet energy can be due to both physics effects (e.g. radiation that is not captured in the jet or the underlying assumption in the methods that events have a pure dijet topology) and to calibration and detector effects. The techniques used in ATLAS aim to only account for the calorimeter-related jet energy fluctuations in the evaluation of the jet energy resolution, and verify in data the assumptions that involve the fluctuations of the jet energy due to physics effects as simulated in Monte Carlo.

\section{Dijet balance method}

The dijet balance method [117] relies on energy conservation in the transverse plane in the case of dijet events. Events with such topology are selected by applying a cut on the azimuthal angle between the two leading jets $\Delta \phi$ so that they are back to back, and limiting the presence of significant third jet radiation. Further details on this technique can be found in Appendix A.1. The total systematic uncertainty for the dijet balance method amounts to $4-6 \%$ in the $p_{\mathrm{T}}^{\text {jet }}$ range of $20-500 \mathrm{GeV}$.

\section{Bisector method}

The bisector method [118] uses the decomposition of the vector sum of the two leading jet four momenta $\mathbf{P}_{\mathbf{T}}$ to estimate the jet energy resolution. The jet transverse momentum resolution is estimated using the variances of the projections of $\mathbf{P}_{\mathbf{T}}$, one on the axis that bisects the angle between the two jets and the other on the axis orthogonal to it. This estimate uses the underlying assumption that at particle level, the two components of the imbalance vector will have equal fluctuations, and further imbalances are due to fluctuations from calorimeter effects. The event selection follows that of the dijet balance method to select good dijet topologies, but removing the cut on the angle between the two jets. Further details on this technique can be found in Appendix A.1. The total systematic uncertainty for the bisector method amounts to $3-4 \%$ in the $p_{\mathrm{T}}^{\text {jet }}$ range of $20-500 \mathrm{GeV}$.

\subsubsection{Results from in-situ methods and comparison to Monte Carlo}

The fractional jet transverse momentum resolution can be parameterised as: 


$$
\frac{\sigma_{p_{T}}}{p_{T}}=\frac{a}{p_{T}} \oplus \frac{b}{\sqrt{p_{T}}} \oplus c
$$

where the first term includes the stochastic sources of fluctuations that are independent of the energy but whose relative impact decreases linearly with energy (e.g. electronics noise, remaining offset from pile-up), the second term includes all all sources that are of statistical nature and follow Poisson statistics (e.g. signal sampling), and the constant term is related to detector effects that are a constant fraction of energy.

The fractional resolution derived from the Monte Carlo simulation with each of the two methods can be fitted using the parameterisation above, and compared to the fractional resolution in data. The result is shown for the bisector method in Figure 4.1 for the calibration schemes described in Section 3.2.2: the Local Cluster Weighting (LCW), Global Cell Weighting (GCW), the Global Sequential calibration (GS) and the EM+JES scheme.

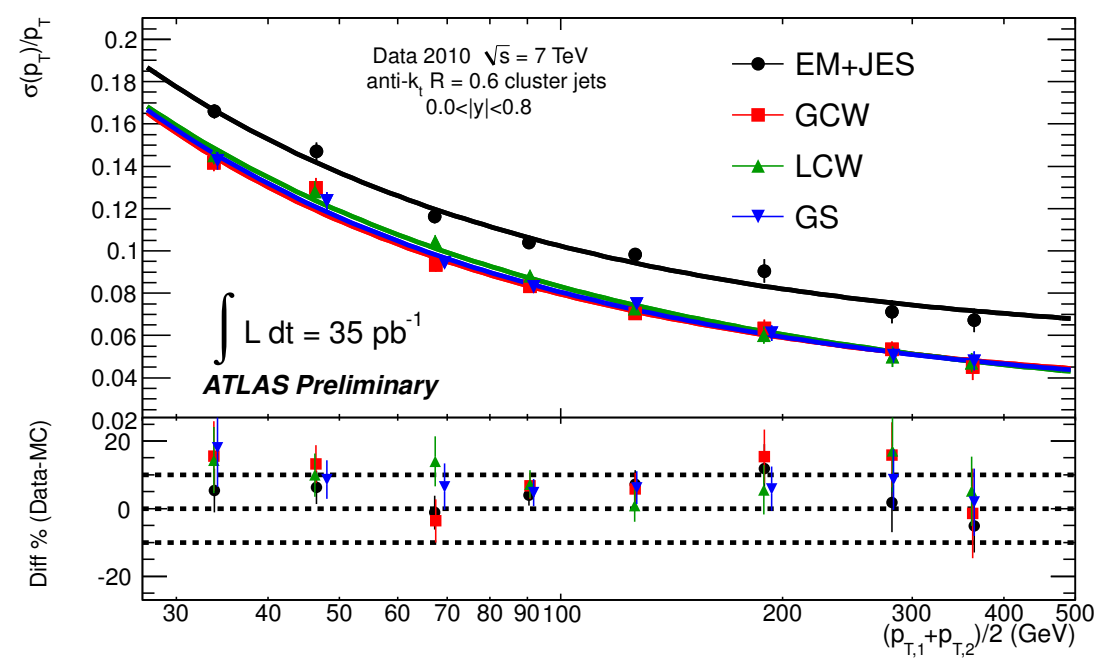

Figure 4.1.:Jet energy resolution estimated using the bisector technique, overlaid for data (points) and Monte Carlo (line) for the calibration schemes described in Section 3.2.2: the Local Cluster Weighting (LCW), Global Cell Weighting (GCW), the Global Sequential calibration (GS) and the EM+JES scheme. The lower ratio plot shows the relative difference between the Monte Carlo parameterisation and the data points. Figure from [116].

The results for both methods in data are compatible with the Monte Carlo simulations within the given systematic and statistical uncertainties of about $10 \%$. The final systematic uncertainty on the jet resolution for each rapidity region is assigned from the weighted average of the systematic errors on the relative data/Monte Carlo difference, and it is flat as a function of $p_{T}$. 


\section{Choice of jet energy resolution parameterisation and its uncertainties}

The agreement for the in-situ results in data and Monte Carlo gives confidence that the truth Monte Carlo resolution can be trusted to represent the resolution in data. The parameterisation of the Monte Carlo resolution from [116] (also shown as the blue line of Figure 4.8 in the case of anti- $k_{t}$ jets with $R=0.6$ ) is taken as a baseline for all ATLAS analyses and it will be referred in the following as the default ATLAS jet energy resolution. In this analysis no isolation cut is applied on the jet, only the leading jets are considered and the fitting range for the parameterisation is $30<p_{\mathrm{T}}^{\text {jet }}<500 \mathrm{GeV}$ since this is the range where in-situ data is available.

In the kinematic range covered by the in-situ data (jets with $30 \leq p_{T}<500 \mathrm{GeV}$ and $|y|<$ 2.8), the comparison of the jet resolution measured with in-situ techniques in data and Monte Carlo shows agreement within the systematic uncertainties of less than 10\%, as mentioned above. Outside the kinematic range of in-situ measurements, the Monte Carlo parameterization is kept but the uncertainty is conservatively increased as follows:

- Jets with $p_{T}=10 \mathrm{GeV}$ are assigned an uncertainty that is three times that of jets with $p_{T}=30 \mathrm{GeV}$. This covers the differences in the jet resolution when the Monte Carlo parameterization is derived taking into account resolution values starting from $15 \mathrm{GeV}$ with respect to the default starting value of $30 \mathrm{GeV}$ (as detailed in Section 4.3.3). The uncertainty varies smoothly from its value at $10 \mathrm{GeV}$ to the value at $30 \mathrm{GeV}$, as a function of $p_{T}$.

- Jets with $p_{T}<1000 \mathrm{GeV}$ are assigned an uncertainty that is twice as much that of jets with $p_{T}=500 \mathrm{GeV}$, with a linearly varying uncertainty between the two values.

- Jets with $p_{T}>1000 \mathrm{GeV}$ are assigned the uncertainty of jets with $p_{T}=1000 \mathrm{GeV}$.

\subsection{In-situ validation of the jet energy scale calibration}

Jet reconstruction, and in particular the jet energy scale calibration based on Monte Carlo, can be validated using in-situ techniques comparing the first $35-38 \mathrm{pb}^{-1}$ of 2010 ATLAS data and the Monte Carlo simulation results. In-situ techniques use well measured benchmark objects that balance the jet to evaluate the relative and absolute jet energy scale. The transverse momentum balance in two-jet QCD events is used to check the uniformity of the calibration in pseudorapidity with the $\eta$ intercalibration method. The absolute energy scale at low jet $p_{T}$ is validated exploiting the $p_{T}$ balance of the jet with well-measured photons in the direct balance and missing- $E_{T}$ projection fraction (MPF) methods [119], while the balance between 
the leading jet and the recoil system is used as a probe for high- $p_{T}$ jets [120]. Jets made of tracks measured in the inner detector can also be compared to the corresponding jet measured from calorimeter energy deposits [121].

This section describes in detail the method to evaluate the relative intercalibration in data, as it is an important ingredient in the evaluation of the jet energy scale uncertainty. More details and data/Monte Carlo comparison plots to validate the jet energy scale calibration using the other in-situ techniques can be found in the references mentioned above and in Appendix A.2.

\subsubsection{Dijet balance}

As shown in Fig. 3.5, the calorimeter response at the electromagnetic scale is not uniform in pseudorapidity due to different material upstream of the calorimeters and to the varying calorimeter technology. The EM+JES calibration equalizes the jet response in both energy and pseudorapidity as shown in Section 4.3, but the complex calorimeter geometry of the ATLAS detector and shifts in the baseline electromagnetic scale that are the starting point for the EM+JES calibration might not be reflected perfectly in the Monte Carlo simulation. For this reason, the relative intercalibration of jets in different pseudorapidity regions needs to be studied in data.

The relative response of endcap to central jets is evaluated using the $p_{T}$ balance techniques described in $[55,122]$. These techniques employ di-jet events to balance the transverse momentum of a probe jet with the $p_{\mathrm{T}}^{\text {jet }}$ of a reference jet, exploiting the momentum balance due to transverse momentum conservation. In such events, the response of the forward jets is measured relative to that of the better measured central jets. The measurement is performed using the 2010 ATLAS dataset, as well as for several MC generator event samples.

In the standard intercalibration method, a fixed reference region is chosen in the central calorimeter region $(|\eta|<0.8)$. Jets falling within this pseudorapidity range are treated as the reference jets, while jets outside this region are considered as probe jets. Figure 4.2 (a) shows a schematic representation of the jet topology studied in the intercalibration method.

The asymmetry $A$ is defined as twice the ratio between the difference and the sum of the transverse momenta of probe and reference jets:

$$
A=2 \frac{p_{T}^{\text {probe }}-p_{T}^{\text {ref }}}{p_{T}^{\text {probe }}+p_{T}^{\text {ref }}} .
$$


The asymmetry distribution is then used to measure the average response of the probe jets relative to the reference jet, in bins of $\eta$ of the probe jet and of the average transverse momentum of the two jets $\left(p_{T}^{\text {avg }}\right)$ :

$$
\frac{p_{T}^{\text {probe }}}{p_{T}^{\text {ref }}}=\frac{2+A}{2-A}=1 / c
$$

Only events where the two leading jets have a $p_{T}^{\text {avg }}>20 \mathrm{GeV}$ are selected. Cuts on the third jet transverse momentum and on the angle between the two leading jets used in the analysis are applied in order to reject topologies with relevant third jet radiation.

The so-called Matrix method employs a similar technique to evaluate the relative jet response but overcomes the necessity of having a jet in the reference region, therefore increasing the available statistics. The definition of probe and reference jets is replaced by left and right jets depending on the relative pseudorapidity (where $\eta^{\text {left }}>\eta^{\text {right }}$ ), as shown in Figure 4.2 (b).

$[! h]$

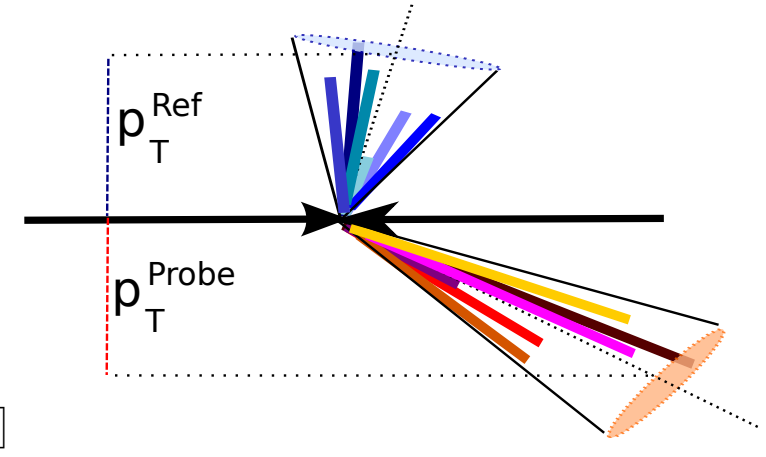

(a)

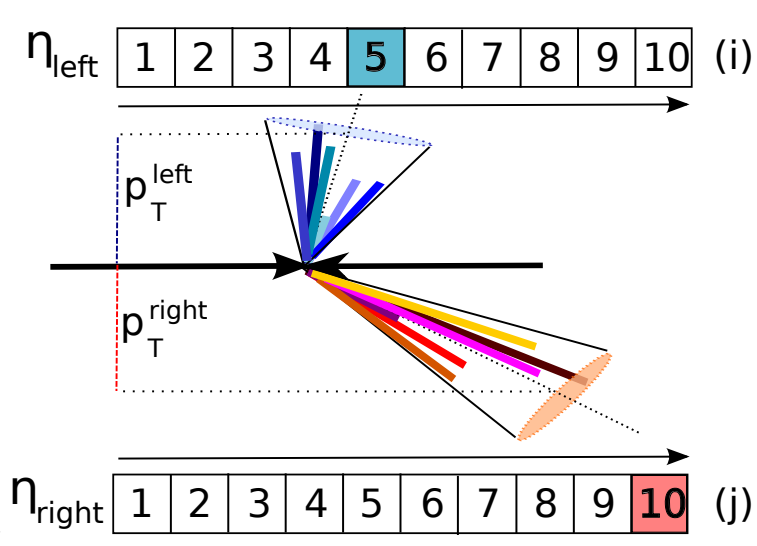

(b)

Figure 4.2.:Figure(a): Schematic representation of the reference and probe jets in the dijet topology used for the calorimeter intercalibration, balanced in transverse momentum. Figure (b): schematic representation of the reference and probe jets in the dijet topology used for the Matrix intercalibration. The jet pseudorapidity regions increase towards the right and they are labeled with integer indices called $i$ and $j$ in the following.

Equations 4.3 and 4.2 still hold for the Matrix method when replacing the reference jet with the rightmost jet and the probe jet with the leftmost one. The average response ratio distribution $\mathcal{R}_{\text {left,right }}=\frac{p_{T}^{\text {jet,left }}}{p_{T}^{\text {jet,right }}}$ is evaluated for each bin of $\eta^{\text {left }}$ (called bin $i$ in the following), $\eta^{\text {right }}$ (bin $j$ ) and for each $p_{T}^{\text {avg }}$ bin (bin $k$ ). A system of linear equations for each of the $p_{T}^{\text {avg }}$ 
bins is minimized to obtain the relative correction factors $c_{i k}, c_{j k}$ in each of the $\eta, p_{T}^{\text {avg }}$ bins, taking into account the statistical uncertainty on the relative response $\Delta\left\langle\mathcal{R}_{i j k}\right\rangle$ :

$$
S\left(c_{1 k}, \ldots, c_{N k}\right)=\sum_{j=1}^{N} \sum_{i=1}^{j-1}\left(\frac{1}{\Delta\left\langle\mathcal{R}_{i j k}\right\rangle}\left(c_{i k}\left\langle\mathcal{R}_{i j k}\right\rangle-c_{j k}\right)\right)^{2}+X\left(c_{i k}\right),
$$

The function $X\left(c_{i k}\right)$ is used to suppress deviations from unity of the average corrections as explained in [122]. Each correction factor is then rescaled so that the reference region $|\eta|<0.8$ has a unitary response.

\section{Dijet balance in MC samples}

The relative jet response is also measured in the nominal PYтнIA sample and for three other Monte Carlo samples: QCD jet events simulated in PYTHIA with the Perugia2010 PYTHIA tune, events simulated using the combination of the ALPGEN generator interfaced to HERWIG and Jimmy (Alpgen + HeRWIG + Jimmy) and events simulated using the HeRWIG++ generator. Details on the nominal sample and on the other generators can be found in Section 1.4.2.

The Perugia2010 Pyтнia tune is an independent set of parameters for the PYTHIA generator, derived using mainly hadron collider data with an increased final state radiation to better reproduce the jet shapes and hadronic event shapes using LEP and Tevatron data [123]. In this tune, parameters sensitive to the production of particles with strangeness and related to jet fragmentation have also been adjusted. The ALPGEN + HERwig + JimmY and HeRwig++ samples are used to test the effects of a different modelling of the hard subprocess and soft processes with respect to the nominal PYтнIA sample used to derive the calibration.

\section{Results}

The data/Monte Carlo comparison of the relative response shows that the relative calibration of jets in the ATLAS calorimeters is fairly well understood for the whole calorimeter region for $p_{T}^{\text {avg }}>60 \mathrm{GeV}$ : data and the various Monte Carlo simulations agree to better than $2 \%$ in the central and endcap region $(|\eta|<2.8)$ and to $5 \%$ in the forward region, as shown in the two example plots in Fig.4.3. For lower average transverse momenta, the different Monte Carlo samples show a larger deviation with respect to data: the MC predictions for the relative jet response diverge for low- $p_{\mathrm{T}}$ forward jets, while the data lie between the predictions. This 
effect has been investigated and attributed to residual low- $p_{T}$ effects, modeled differently by the different generators, and further studies are ongoing at the time of writing this thesis.

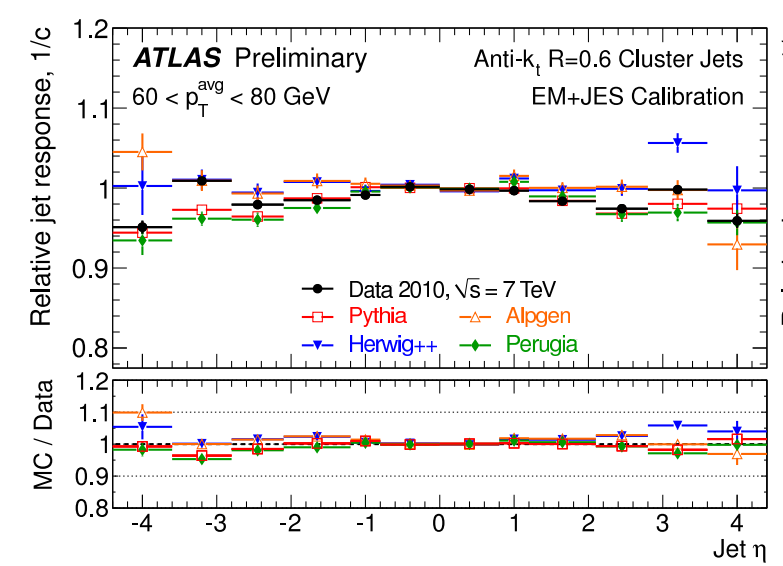

(a)

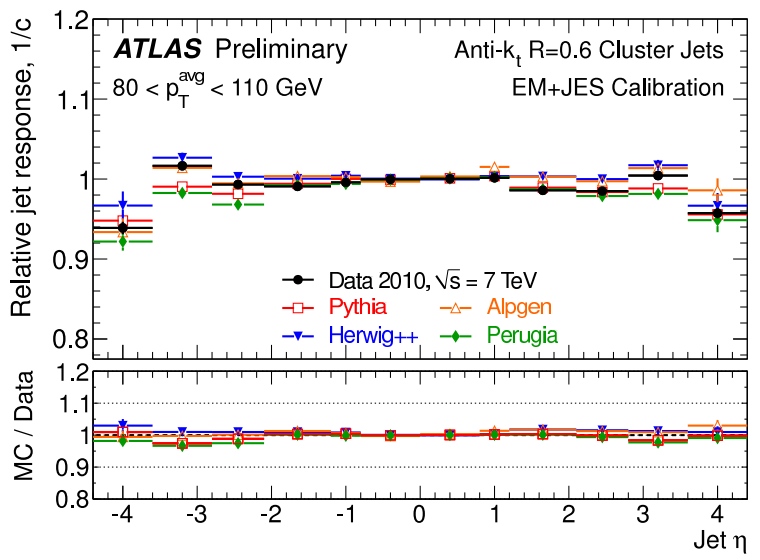

(b)

Figure 4.3.:Relative jet response, $1 / \mathrm{c}$, as a function of the jet pseudorapidity found using the matrix method for $45<p_{T}^{a v g}<60 \mathrm{GeV}$ (a) and $80<p_{T}^{a v g}<110 \mathrm{GeV}$ (b)

\subsection{Monte Carlo based performance studies}

Since the start of data taking, the JeTPERFormance software package in the ATHENA ATLAS framework has been employed for assessing the performance of the jet reconstruction. Plots produced with the JETPERFORMANCE package have been used to validate the correction constants for the various step of the EM+JES calibration scheme that is used as default in both the ATLAS datasets considered in this thesis. More details on this software package are given in Appendix B.

The JETPERFORMANCE package aims to provide the standard infrastructure for physicists in the ATLAS experiment to evaluate the performance of the jet reconstruction. This software compares the reconstructed jets from a chosen sample to a collection of reference objects that can provide Monte Carlo truth benchmarks (such as particle jets built from Monte Carlo simulation), or in-situ energy balance (such as recoiling jets or photons in the same event). The performance of the jet reconstruction is then measured in kinematic and spatial bins in terms of benchmark quantities such as:

- jet response, defined in Equation 3.1 when energy is the chosen kinematic variable;

- jet resolution, defined as the ratio of the width of the distribution of the chosen kinematic variable and its average; 
- jet purity ${ }^{3}$, defined as the ratio between the number of calorimeter jets with a spatially matched Monte Carlo truth jet and the total number of calorimeter jets;

- jet efficiency ${ }^{3}$, defined as the ratio between the number of Monte Carlo truth jets with a spatially matched calorimeter jet and the total number of Monte Carlo truth jets.

A selection of plots produced with the JETPERFORMANCE package for the validation of the jet reconstruction for 2010 data is shown in the following. Only plots for anti- $k_{t}$ with distance parameter 0.6 are shown in the following, with similar results obtained for a distance parameter of 0.4. The jet selection and matching criteria are the same as detailed in Section 3.2.1, unless the plot is labeled as inclusive jets: in that case, no jet isolation cut is applied.

\subsubsection{Energy and $p_{T}$ response}

Figure 4.4,the energy and $p_{T}$ response are shown for jets after the EM+JES calibration in the nominal Monte Carlo sample. In the barrel region $0.3<|\eta|<0.8$ the jet response is restored to about $2 \%$ at low $p_{\mathrm{T}}^{\text {jet }}$ and to better than $1 \%$ for $p_{\mathrm{T}}^{\text {jet }}>30 \mathrm{GeV}$. In the endcap and forward region, the closure to unity is better than $1 \%$ for $p_{\mathrm{T}}^{\text {jet }}>20 \mathrm{GeV}$, while the energy response is within $1 \%$ for jets with transverse momentum above $30 \mathrm{GeV}$. The residual non-closure is taken as a source of systematic uncertainty, as described in Chapter 5.

In Figure 4.5 the $p_{T}$ response for jets built from either topocluster or topotower inputs (the latter are called simply Tower) and calibrated with the various ATLAS calibration schemes is shown in the case of the nominal Monte Carlo sample, for jets measured in the whole detector pseudorapidity $(|\eta|<4.5)$. The average response for all calibrations is restored to the hadronic scale to better than $1 \%$.

\subsubsection{Effect of pile-up on the jet response}

The effect of the pile-up offset correction is shown in Figure 4.6. Here the response for pile-up in a Monte Carlo sample with an average of 5 additional interactions per event is shown, with and without the offset correction. Since truth jets do not contain particles from any additional interaction, the comparison of the EM+JES-calibrated jets with and without the offset correction shows that the correction reduces the pile-up contribution to the jet energy by about $10 \%$ for both topocluster and tower jets. The better performance of the offset correction in the case of tower jets with respect to topocluster jets is due to the averaging of the number of towers present a in topocluster jet for the jet-level correction, while the actual number of towers is used

\footnotetext{
${ }^{3}$ only available in MC comparison
} 


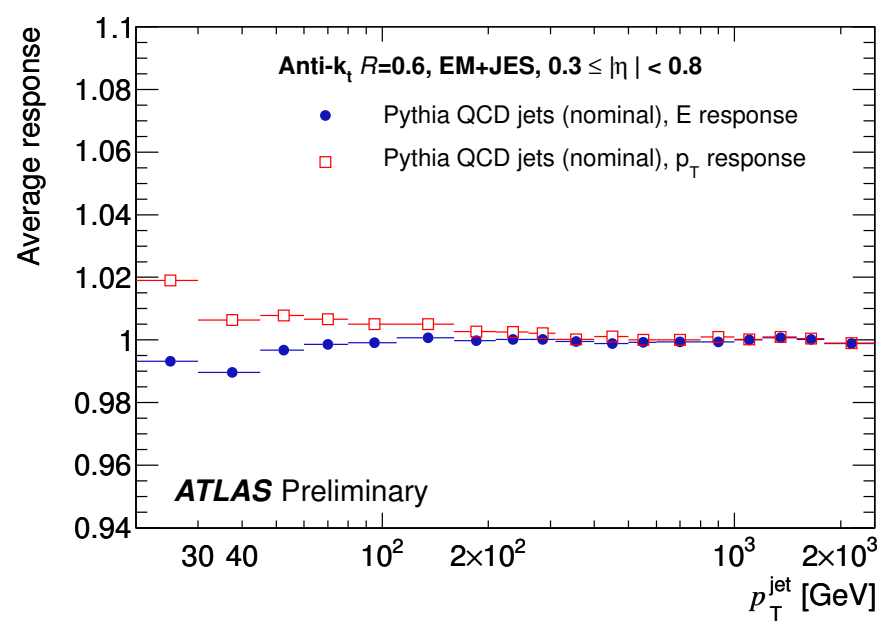

(a)

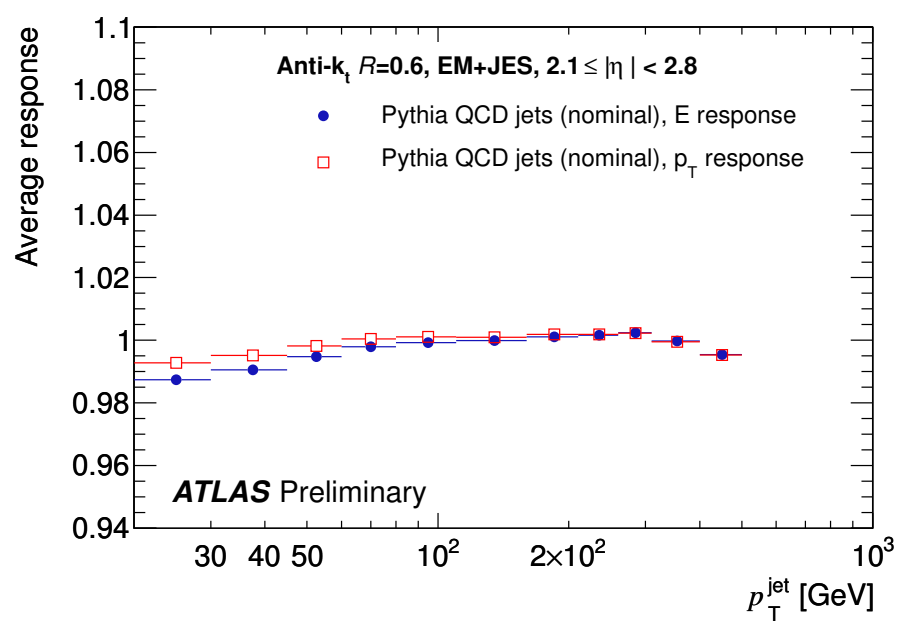

(b)

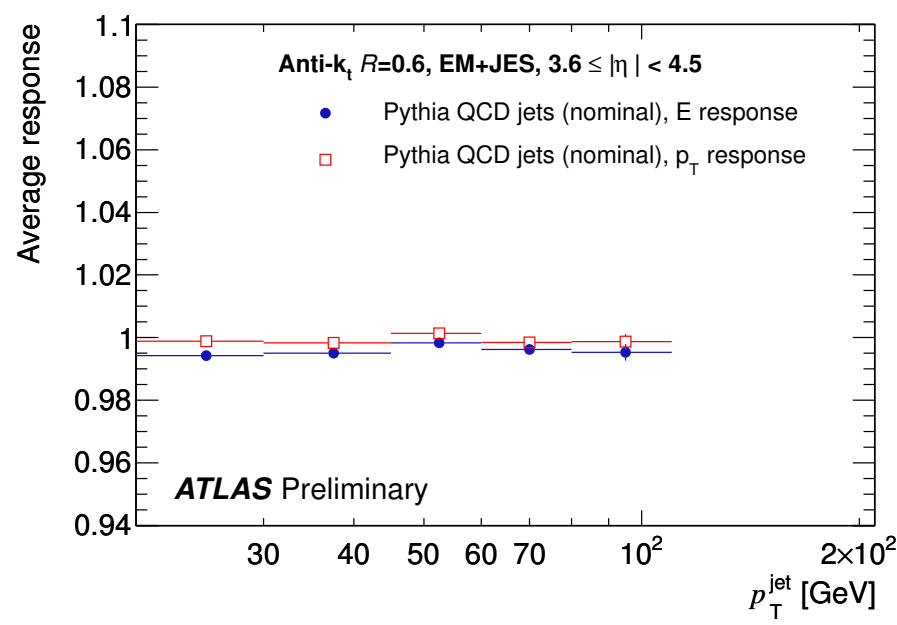

(c)

Figure 4.4.:Simulated jet $p_{T}$ response (full circles) after the EM+JES calibration and jet energy response (open squares) as a function of $p_{\mathrm{T}}^{\text {jet }}$ for the nominal sample for jets in the central (a), endcap (b) and forward (c) calorimeter regions. 


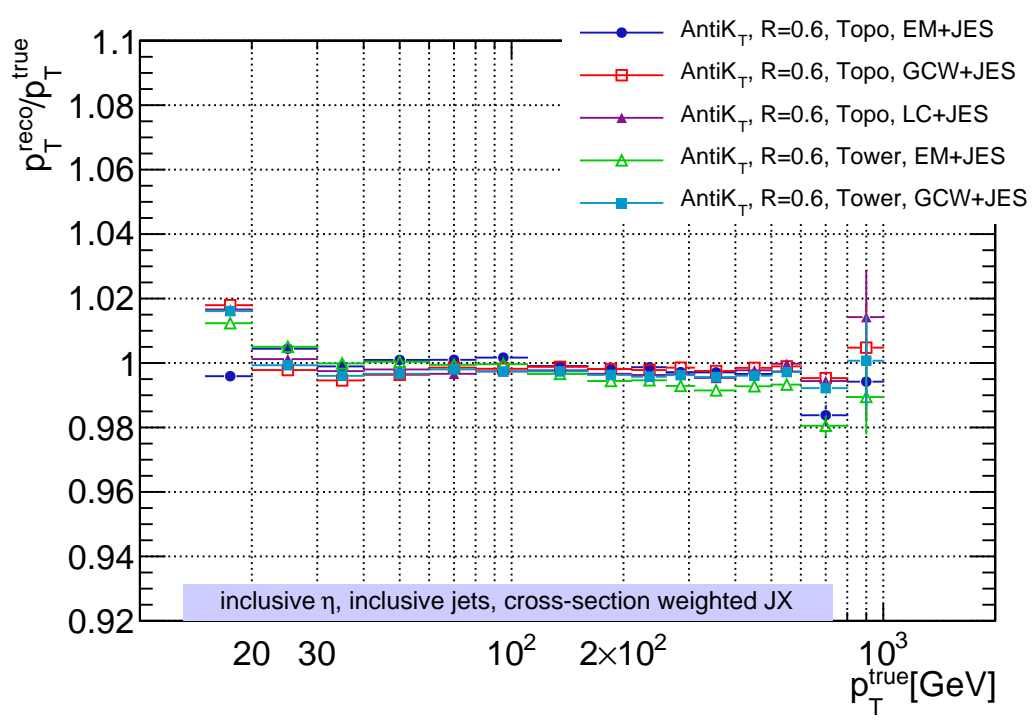

Figure 4.5.:Simulated jet $p_{T}$ response for three of the different ATLAS calibration schemes outlined in Section 3.2.2 (the Local Cluster Weighting (LCW+JES), Global Cell Weighting (GCW+JES), and the EM+JES scheme) for the nominal Monte Carlo sample integrated over all jet pseudorapidities.

as it is available in the case of topotower jets. The effect of pile-up in Monte Carlo is larger than what is effectively observed in data using in-situ techniques because the average number of vertices in the Monte Carlo sample used is higher than in the data, as shown in Figure 2.2.

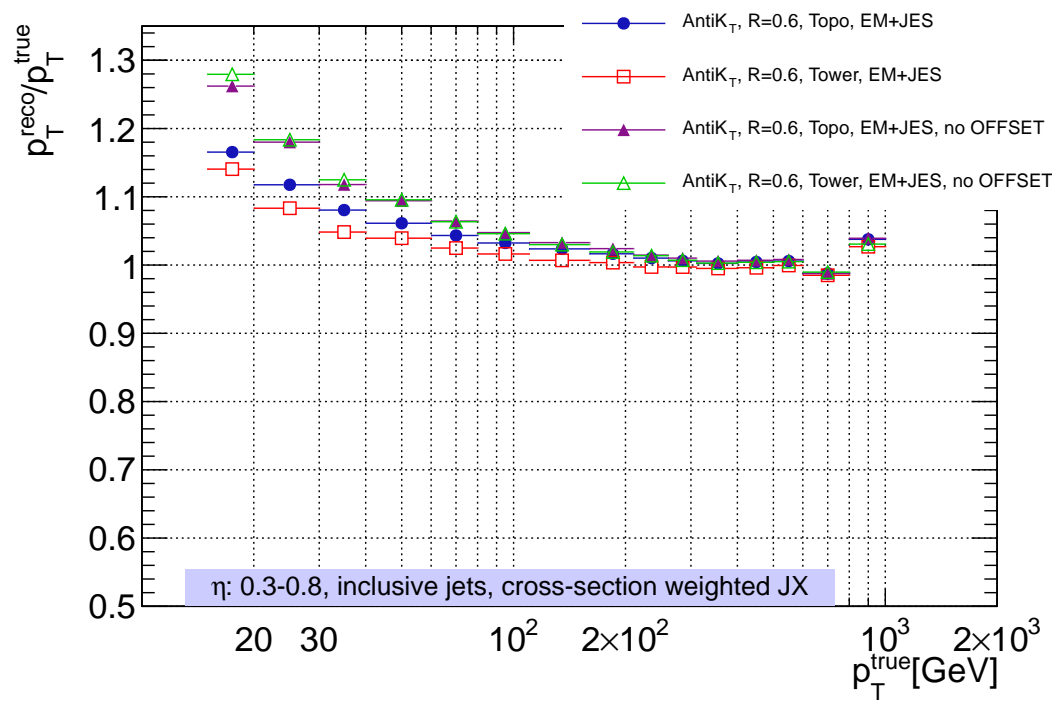

Figure 4.6.:Effect of the pile-up offset correction in the EM+JES calibration chain for anti- $k_{t} R=0.6$ jets in the central region. 


\subsubsection{Jet energy resolution from Monte Carlo}

The fractional $p_{\mathrm{T}}$ resolution for isolated jets in the nominal Pythia Monte Carlo sample for anti- $k_{t} R=0.6$ jets is shown in figure 4.7 . This plot reflects how the transverse momentum resolution decreases with higher jet pseudorapidities, since $\eta$ determines the energy of a jet of a given $p_{T}\left(E \approx \frac{p_{T}}{\cosh |\eta|}\right)$. Since the dominant terms in the fractional resolution at low $p_{T}$ are divided by the energy or by its square root, the jet resolution improves at higher jet pseudorapidities.

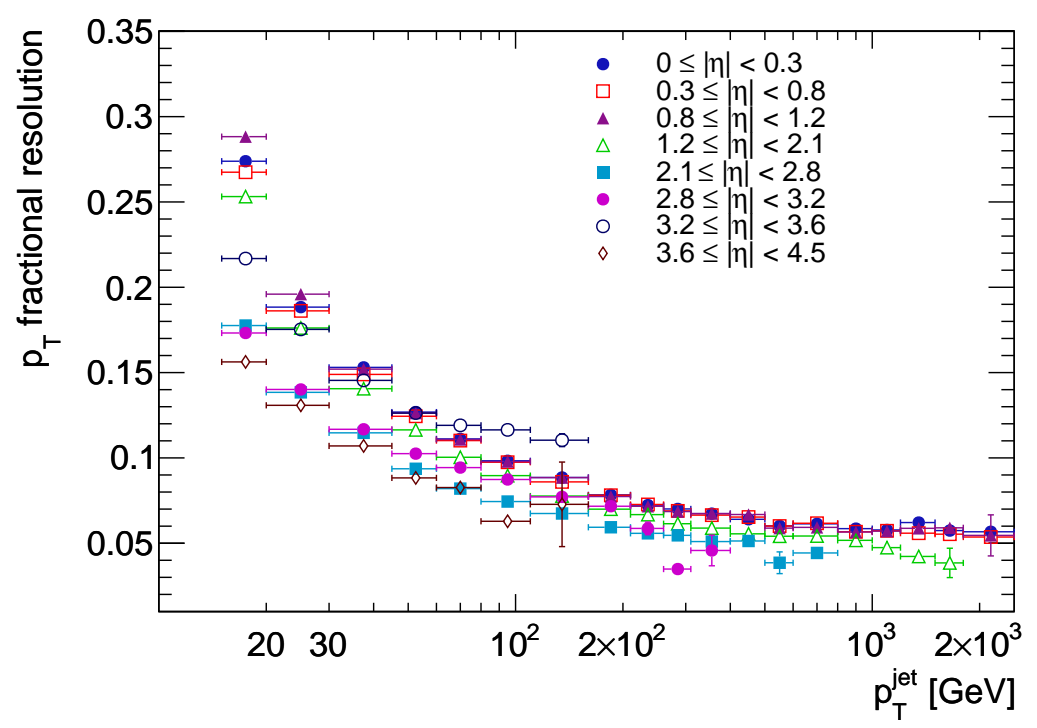

Figure 4.7.:Jet $p_{T}$ resolution in Monte Carlo for EM+JES calibrated anti- $k_{t} R=0.6$ topocluster jets in different pseudorapidity bins.

These results can be fitted with the parameterisation of Equation 4.1 and compared with those obtained in Monte Carlo using the standard jet resolution analysis and described in Section 4.1.2. The main differences between the way the two parameterisations were derived are:

1. Isolated jets are used to estimate the resolution of Figure 4.7, no isolation cut is applied in the standard analysis;

2. All isolated jets in the events are used for Figure 4.7, while only the two leading jets are considered in the standard analysis;

3. The fit range used in the parameterisation is $15<p_{\mathrm{T}}^{\text {jet }}<500 \mathrm{GeV}$ while $30<p_{\mathrm{T}}^{\text {jet }}<500$ $\mathrm{GeV}$ is used for the standard analysis.

For these reasons, the parameterisation derived using the JetPerFormance analysis will not coincide with the default parameterisation, as shown in Figure 4.8. The disagreement is 
particularly evident at low jet $p_{T}$ since no information below $p_{\mathrm{T}}^{\text {jet }}=30 \mathrm{GeV}$ is used in the default fit, and in the endcap and forward region where the number of available points is reduced because of the kinematic limit and the fit is more constrained by the low $p_{T}$ points that are not used in the default fit. To cover this discrepancy and possible systematics due to different event topologies and limited fit range, the error for the default jet resolution parameterisation

below $p_{\mathrm{T}}^{\text {jet }}=30 \mathrm{GeV}$ is increased to cover the Monte Carlo parameterisation as mentioned in Section 4.1.2.

\subsubsection{Monte Carlo purity and efficiency}

Figure 4.9 shows the reconstruction purity and efficiency of EM+JES calibrated topocluster jets in the nominal Monte Carlo sample in the central, endcap and forward detector regions. The isolation cut has been removed for these plots, and the $15 \mathrm{GeV} p_{T}$ cut on truth jets has been lowered to $7 \mathrm{GeV}$ for the purity plot to avoid biasing the matching for reconstructed jets at low $p_{T}$. The jet reconstruction efficiency in the Monte Carlo simulation is above $95 \%$ for the whole kinematic range used in the measurement, while the purity of the reconstructed jet sample is above $94 \%$. Both efficiency and purity grow to unity as the energy of the jets increases for higher pseudorapidities. 


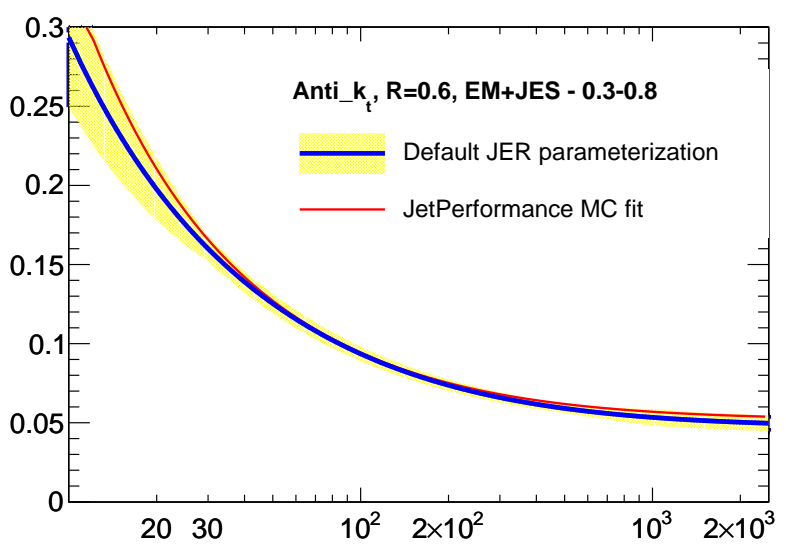

(a)

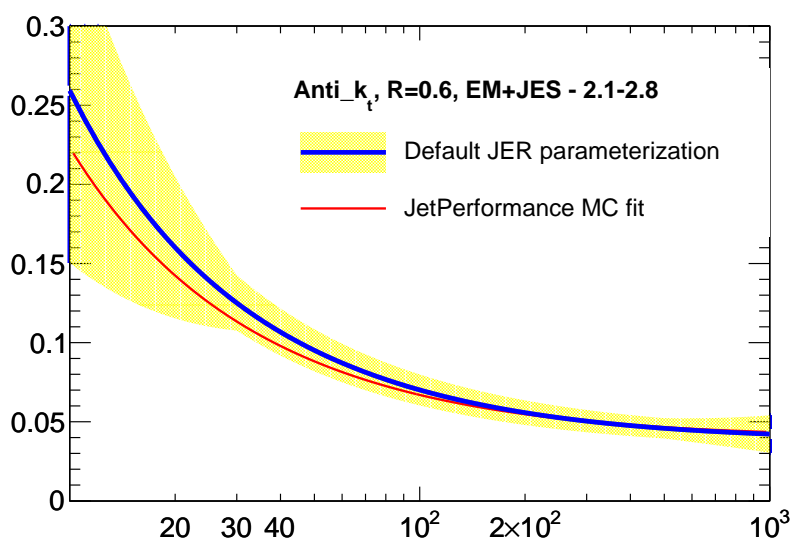

(b)

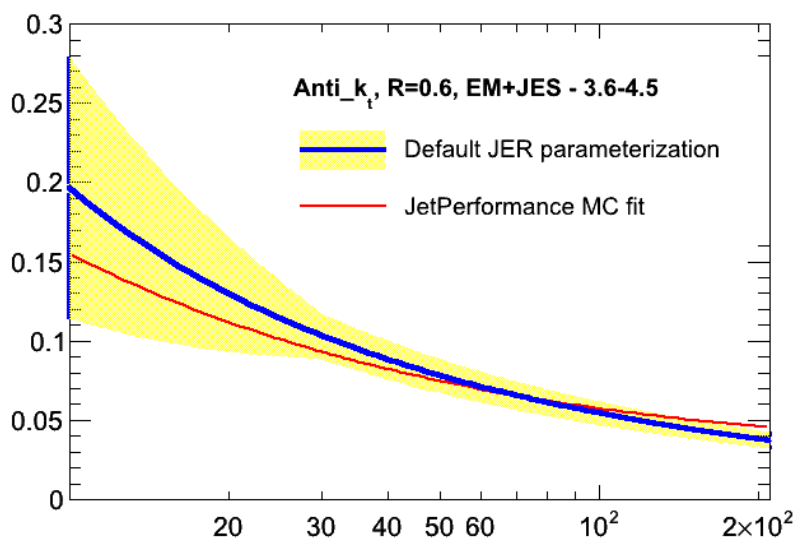

(c)

Figure 4.8.:Default parameterisation of the jet $p_{T}$ resolution as a function of $p_{\mathrm{T}}^{\text {jet }}$ (blue, with yellow band as error) and parameterisation using Monte Carlo information below $30 \mathrm{GeV}$ (red) for the nominal sample for jets in the central (a), endcap (b) and forward (c) calorimeter regions. 


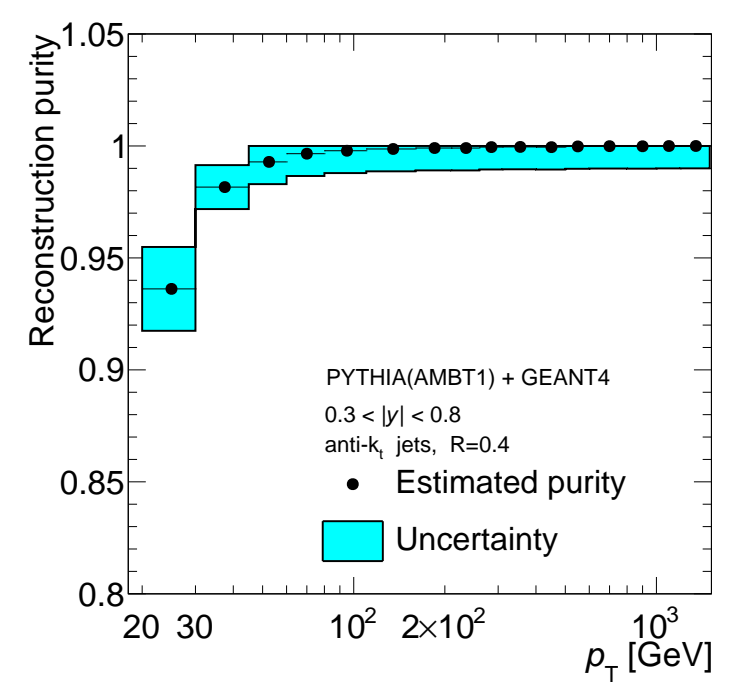

(a)

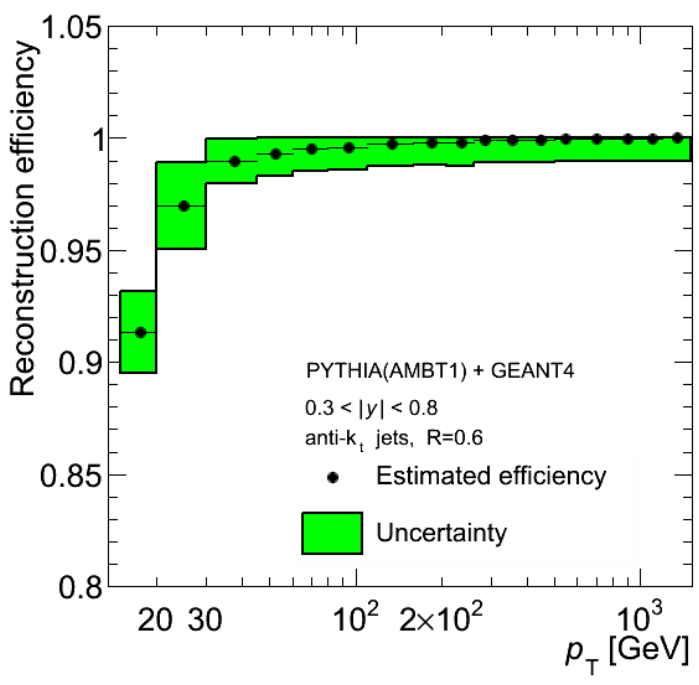

(c)

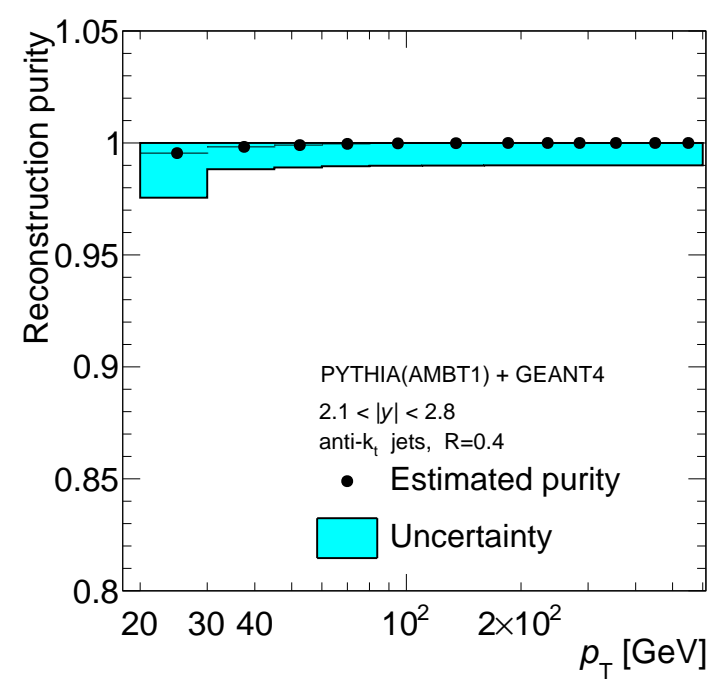

(b)

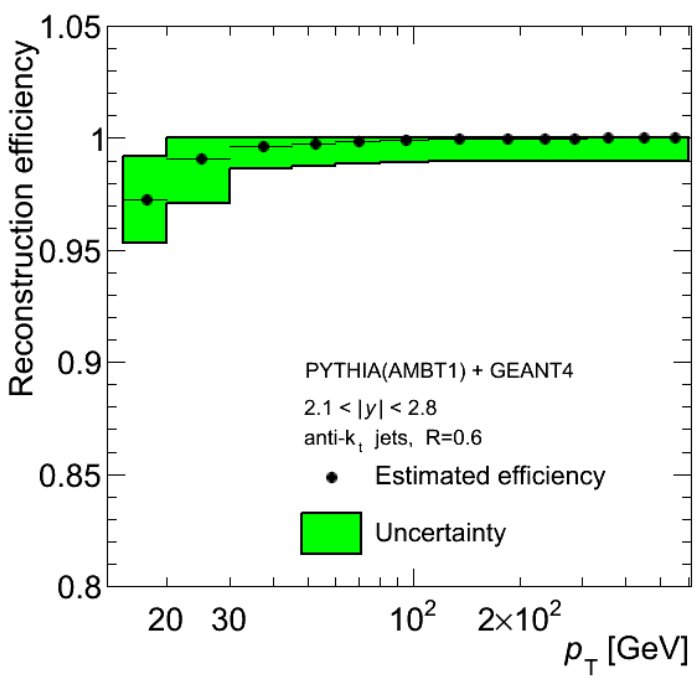

(d)

Figure 4.9.:Purity (a), (b) and efficiency (c), (d) for EM+JES-calibrated anti- $k_{t} R=0.6$ topocluster jets in the nominal Monte Carlo sample in the central and endcap rapidity regions. Plots made in collaboration with [124]. 


\section{Chapter 5.}

\section{Jet Energy Scale Uncertainty}

The uncertainty of the measurement of the jet kinematics after the calibration (jet energy scale uncertainty, or JES uncertainty) is the dominant experimental uncertainty for numerous physics results and for the inclusive jet cross section measurement discussed in this thesis.

A first estimate of the JES uncertainty in the ATLAS detector was provided as described in Reference [125] and summarised in Appendix D. Its evaluation was largely based on information available before the first LHC collisions and it exploited transverse momentum balance in di-jet events. The calibration and the JES uncertainty were updated for the full 2010 dataset, as described in References [111,126]. The EM+JES calibration scheme described in Section 3.2.1 has been used to calibrate jets. Data-derived techniques have been employed to correct the calorimeter response and estimate its uncertainty. These include the calibration of the absolute electromagnetic energy scale of the calorimeters from the analysis of $Z$-boson decays in the electron channel $(Z \rightarrow e e)$ [78] as described in Section 2.5.1, precise in-situ measurements of the single hadron response $[48,49]$ and measurements of the calorimeter cell noise suppression uncertainty from data. Employing in-situ techniques allows a significant reduction of the JES uncertainty in the central detector region with respect to the previous estimate. Confidence in a reduced JES uncertainty is gained from the increased knowledge of the detector performance obtained during the analysis of the first year of ATLAS data [48-55] and from the results from the JES validation using in-situ techniques detailed in Section 4.2.

This chapter is concerned with the full 2010 calibration and JES uncertainty analysis and will only show results for EM+JES calibrated jets built with a distance parameter of $R=0.6$, unless otherwise noted. The outline of this chapter is as follows: Section 5.1 describes the details of the analysis employed for the estimate of the jet energy scale uncertainty, such as the jet selections applied to the Monte Carlo simulation and data samples. The sources of systematic uncertainties for the jet energy scale and their derivation using simulated and collision data are 
detailed in Section 5.2. The effect of pile-up on the jet energy scale and its related uncertainty is discussed in Section 5.3. The combination of the individual uncertainty contributions is described in Section 5.4. Results, and their validation using in-situ techniques, are in Section 5.5. Considerations on the applicability of the estimate of the JES uncertainty to different event topologies and flavour compositions are reported in Section 5.6.

\subsection{Analysis details}

The JES systematic uncertainty is derived combining information from in-situ and single pion test-beam measurements, uncertainties on the material budget of the ATLAS detector, the description of the electronic noise, and the Monte Carlo modelling used in the event generation. Test samples with differing conditions with respect to the the nominal Pythia QCD Monte Carlo sample described in Section 3.2 are employed to provide an estimate of the systematic effects contributing to the JES uncertainty. The energy scale of jets for the nominal and the systematic Monte Carlo samples is calibrated using the EM+JES scheme (3.2.1).

The JES uncertainty is provided for jets with transverse momentum greater than $20 \mathrm{GeV}$ and in the full pseudorapidity range of $|\eta|<4.5$ in which jets are measured in ATLAS. The JES uncertainty is estimated as a function of calibrated jet transverse momentum $\left(p_{\mathrm{T}}^{\mathrm{jet}}\right)$ and pseudorapidity $\eta$. The $p_{T}$ binning follows the choices made for the inclusive jet cross section measurement of Chapter 6 . The pseudorapidity bins used for the estimate of the JES uncertainty divide the ATLAS detector in the seven $\eta$ regions specified in Table 5.1 and following the nomenclature used in Fig. 3.5.

Table 5.1.:Detector regions and corrected pseudorapidity bins used for the estimate of the JES uncertainty.

\begin{tabular}{|c|c|}
\hline $\boldsymbol{\eta}$ region & ATLAS detector regions \\
\hline $0<|\eta| \leq 0.3$ & Central (Barrel) \\
$0.3<|\eta| \leq 0.8$ & \\
\hline $0.8<|\eta| \leq 1.2$ & Endcap \\
$1.2<|\eta| \leq 2.1$ & (Barrel-Endcap Transition and HEC) \\
$2.1<|\eta| \leq 2.8$ & \\
\hline $2.8<|\eta| \leq 3.2$ & Transition (HEC-FCal Transition) \\
\hline $3.2<|\eta| \leq 3.6$ & \\
$3.6<|\eta| \leq 4.5$ & Forward (FCal) \\
\hline
\end{tabular}


The JES systematic uncertainty for all jets with pseudorapidity beyond $|\eta|=0.8$ is determined using the JES uncertainty for the central barrel region $(0.3<|\eta|<0.8)$ as a baseline ${ }^{1}$, and adding a contribution from the relative calibration of the jets with respect to the central barrel region. This choice is motivated by the better knowledge of the detector geometry in the central region, and by the use of test-beam measurements only extending to the Tile calorimeter barrel for the estimate of the calorimeter response uncertainties.

\subsubsection{Selection of jets for systematic studies in Monte Carlo studies}

For the JES uncertainty studies the jet energy response at the calibrated energy scale is obtained for the nominal and each of the alternative Monte Carlo samples using reconstructed calorimeter jets matched to $\mathrm{MC}$ truth particle jets within a cone of $\Delta R=0.3$. Only $\mathrm{MC}$ truth jets with $p_{\mathrm{T}}^{\text {truth }}>15 \mathrm{GeV}$ and calorimeter jets with a $p_{\mathrm{T}}^{\text {jet }}>7 \mathrm{GeV}$ after calibration are considered.

The JES uncertainty components derived from simulation are obtained by studying the average calorimeter energy response of calibrated jets for the nominal and each of the alternative Monte Carlo samples. This average response, defined as $\langle\mathcal{R}\rangle=\left\langle E_{\text {calo }}^{\mathrm{EM}+\mathrm{JES}} / E_{\text {truth }}\right\rangle$ or $\langle\mathcal{R}\rangle=$ $\left\langle p_{\mathrm{T}}^{\text {jet }} / p_{\mathrm{T}}^{\text {truth }}\right\rangle$, is obtained by matching isolated calorimeter jets to MC truth jets as described in Section 3.2.1, but excluding the isolation cut for truth jets ${ }^{2}$. The JES systematic uncertainty is derived for isolated jets in the full 2010 dataset, so that the topology dependence of the jet energy scale in the presence of close-by jets can be factorised and the uncertainty considered separately, as explained further in Section 5.6.

The calibrated response $\langle\mathcal{R}\rangle$ is studied in bins of truth jet transverse momentum $p_{\mathrm{T}}^{\text {truth }}$. The $p_{\mathrm{T}}^{\text {truth }}$ corresponding to the bin centre is transformed on average to the calibrated $p_{\mathrm{T}}^{\text {jet }}$ value using a simple inversion procedure ${ }^{3}$. The shifts between the Monte Carlo truth level $p_{\mathrm{T}}^{\text {truth }}$ bin centres and the reconstructed $p_{\mathrm{T}}^{\text {jet }}$ bin centres are negligible with respect to the chosen $p_{T}$ bin widths. Hence the average jet response can be obtained to good approximation as a function of $p_{\mathrm{T}}^{\text {jet }}$.

\footnotetext{
${ }^{1}$ This is the largest fully instrumented region where test-beam information is available, as detailed in Section 5.2.2.

${ }^{2}$ The truth isolation cut has a negligible impact on the average jet response given that truth jets are matched to isolated reconstructed jets.

${ }^{3}$ This means that one multiplies the value of the centre of the bin (in terms of truth jet $p_{T}$ ) by the average response so to transform the coordinate of the $\mathrm{x}$ axis into $p_{\mathrm{T}}^{\mathrm{jet}}$. This procedure is described in detail in Reference [127].
} 


\subsection{Sources of jet energy scale uncertainty}

This section focuses on the description of the sources of systematic uncertainties and their effect on the response of EM+JES calibrated jets. The contributions to the JES systematics can be divided in the categories below:

1. the uncertainty due to the JES calibration method (as described in Section 5.2.1);

2. the uncertainty due to the calorimeter response (Section 5.2.2);

3. the uncertainty due to the detector simulation (Section 5.2.3);

4. the uncertainty due to the physics model and parameters employed in the Monte Carlo event generator (Section 5.2.4);

5. the uncertainty due to the relative calibration for jets with $|\eta|>0.8$ (Section 5.2.5).

\subsubsection{Uncertainty due to the JES calibration}

After the nominal inclusive jet Monte Carlo simulation sample is calibrated with the JES correction constants that were derived from the sample itself, the jet energy and $p_{T}$ response still shows slight deviations from unity at low $p_{T}$ (non-closure). Figure 4.4 in Section 3.2.1 shows the jet response for $p_{T}$ and energy as a function of $p_{\mathrm{T}}^{\text {jet }}$ for the nominal Monte Carlo sample in the barrel and endcap regions for anti- $k_{t}$ jets with $R=0.6$.

Any deviation from unity (non-closure) in $p_{\mathrm{T}}^{\mathrm{jet}}$ and energy response after the application of the JES to the nominal Monte Carlo sample implies that the kinematics of the calibrated calorimeter jet are not restored to that of the corresponding particle jets. This is mostly due to using the same correction factor for energy and transverse momentum. In the case of a non-zero jet mass that does not reflect the truth jet mass, restoring only the jet energy and pseudorapidity will lead to a bias in the $p_{T}$ calibration. Further biases in the energy response at low $p_{T}$ can be due to the jet thresholds used to derive and to apply the constants, respectively 7 $\mathrm{GeV}$ at a JES calibrated with previously obtained calibration constants ${ }^{4}$ and $10 \mathrm{GeV}$ at the electromagnetic scale. The use of cross section weights for the separate samples, considered only

\footnotetext{
${ }^{4}$ This is a 'bootstrapping' problem since jets that are used to derive new calibration constants still must satisfy the $p_{T}>7 \mathrm{GeV}$ reconstruction threshold. A solution to this could be to decrease the reconstruction threshold and/or use two iterations for deriving the calibration so that all jets with $p_{T}>7 \mathrm{GeV}$ used for physics analysis are correctly calibrated, but it wasn't considered practical in the timescale needed to provide the calibration constants for the reprocessing.
} 
in the JES uncertainty analysis, also influences the results of the fit to the response distribution and can lead to non closure at low $p_{T}$.

The systematic uncertainty due to the non-closure of the nominal JES calibration is taken as the largest deviation of the response from unity between energy and $p_{\mathrm{T}}$.

Given that the same JES calibration constants are applied to all samples with systematic variations used for the derivation of the JES uncertainty, the correlation of the non-closure term with the other uncertainty components needs to be considered. The non-closure uncertainty is considered uncorrelated to the other JES uncertainty components, given that:

1. the JES calibration constants vary slowly within the $p_{T}$ bins used for the estimate of the JES uncertainty;

2. the systematic variations produce only small effects on the jet $p_{T}$ spectrum within a bin.

For these reasons, the variation of the calibration constants due to systematic effects can be factorized from the variation of the jet energy. This variation will effectively cancel when taking the ratio of the jet response for any of the systematic samples and the jet response in the nominal sample. The non-closure term becomes then the only uncertainty source where the variation of the calibration constants is accounted for: it can therefore be considered uncorrelated to the other uncertainty contributions and added in quadrature to evaluate the total JES systematic uncertainty.

\subsubsection{Uncertainty on the calorimeter response}

The response and corresponding uncertainties for single particles interacting in the ATLAS calorimeters can be propagated to the energy deposits comprised by jets. This can then be used to derive the calorimeter jet energy scale uncertainty in the central calorimeter region, as detailed in References [48, 49, 128].

Test-beam data and ATLAS data at $900 \mathrm{GeV}$ and $7 \mathrm{TeV}$ are employed to evaluate the uncertainties on the calorimeter response for single particles. The in-situ measurement of the single particle response reduces significantly the uncertainties due to the limited knowledge of the exact detector geometry, in particular those due to the presence of additional dead material, and the modeling of the hadronic interactions of particles in the detector. 


\section{Calorimeter response uncertainty from single hadron response studies}

The ATLAS simulation infrastructure is able to link the true calorimeter energy deposits in each calorimeter cell to the generated particles. The jet calorimeter response uncertainty can be obtained from the uncertainty on the response of each particle in the jet in simulated events. In this way the jet response can be deconvoluted from the response of the individual particles forming jets and the JES uncertainty can be determined using the single particle response uncertainties.

The following single particle response measurements are used to estimate the single particle uncertainties:

- the single hadron energy measured in the ATLAS detector for a cone around an isolated track with respect to the track momentum $(E / p)$ in the momentum range from $0.5<p<20 \mathrm{GeV}$,

- the pion response measurements performed in the 2004 combined ATLAS test-beam, where a full slice of the ATLAS detector has been exposed to pion beams with momenta between 20 and $350 \mathrm{GeV}$ [129].

\section{Uncertainty contributions for single particle energy deposits}

The contributions to the uncertainties of the single particle energy deposits used in the pseudoexperiments are listed below. The names in bold in the following list will be used to characterise the single subcomponents of the calorimeter uncertainty in the following.

$\boldsymbol{E} / \boldsymbol{p} E / p$ studies in the ATLAS detector evaluate the calorimeter response for single isolated hadrons. For particles below $20 \mathrm{GeV}$, the discrepancy between data and Monte Carlo simulation from the $E / p$ analysis of $7 \mathrm{TeV}$ data observed in Reference [128] is taken as the systematic uncertainty for the energy deposit of each charged particle composing the jet, and the statistical error on the measurement is considered as an input for the pseudo experiments. The bottom panel of Figure 5.1 shows the $E / p$ data/Monte Carlo ratio in a sample $\eta$ and $p_{T}$ bin and the systematic uncertainties as the grey band.

$\boldsymbol{E} / \boldsymbol{p}$ acceptance, cluster thresholds Additional uncertainties that could affect the combination of the responses for the single particles within the jet are also taken into account. These effects include energy deposits outside the chosen inclusion radius around the track (leading to a negligible contribution), calorimeter acceptance due to material upstream of the calorimeters and topological cluster threshold effects. 


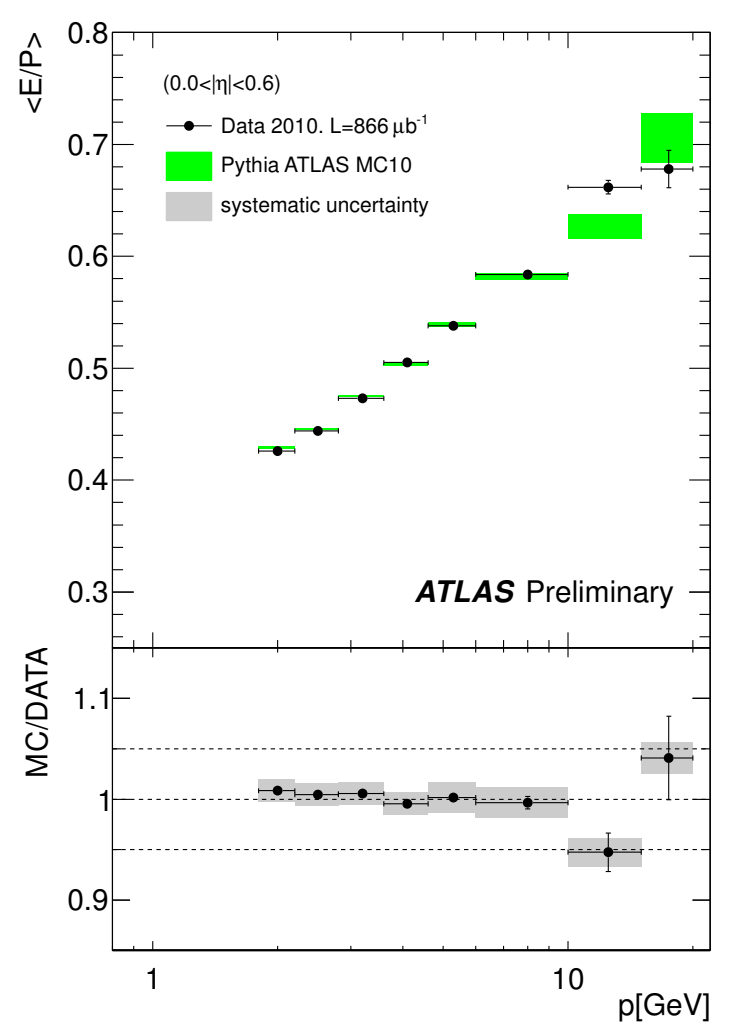

(a)

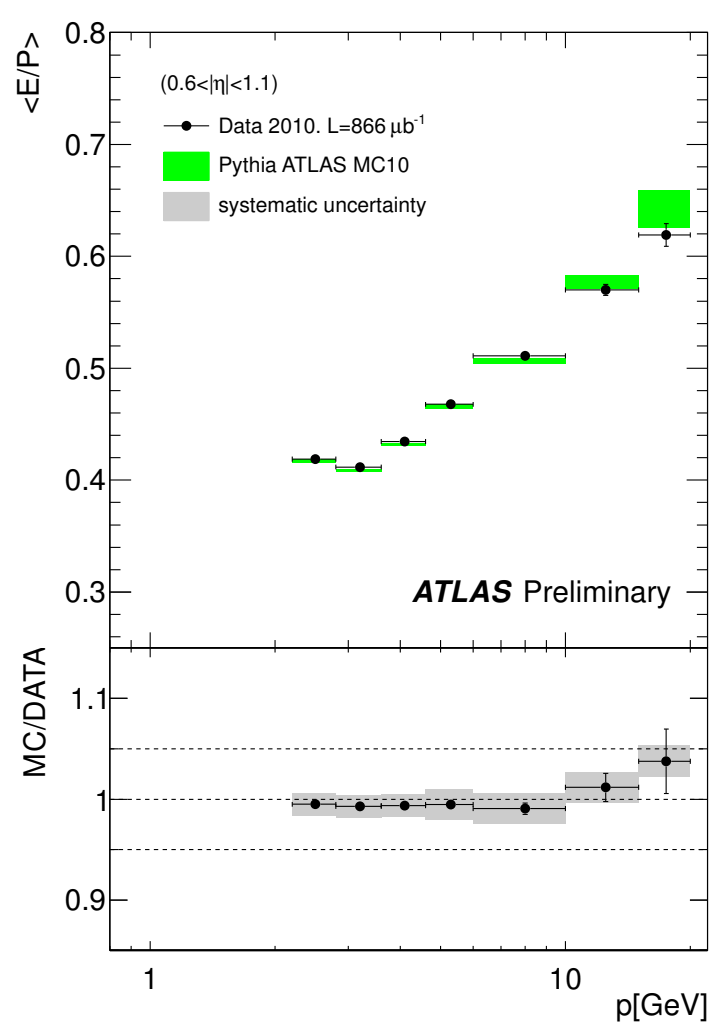

(b)

Figure 5.1.: Mean $E / p$ as a function of the track momentum in two different $|\eta|$ bins (after a background subtraction procedure) for data collected in 2010 and Monte Carlo prediction, and ratio between the MC and data. Figure from [128].

Combined Test Beam (CTB) Test-beam measurements of pions described in Reference [129] are used to estimate the ratio between data and Monte Carlo simulation for charged pions between 20 and $350 \mathrm{GeV}$, with the addition of systematic uncertainties that take into account the difference in the setup of the test-beam and the full ATLAS detector where the single particle analysis is performed.

Neutral particles and antiprotons No test-beam measurements are available for neutral hadronic particles and antiprotons. Specific data and Monte Carlo comparisons and GEANT4 studies have been performed to derive an uncertainty on these particles [95] $\left(K_{s}^{0}\right.$ have not been considered since they mostly decay into pions before reaching the calorimeters).

High momentum particles At high transverse momentum, the dominating contribution to the calorimeter response uncertainties is due to particles with $p>400 \mathrm{GeV}$. The uncertainty for 
these particles has been conservatively estimated as $10 \%$ to take into account calorimeter non linearities and longitudinal leakage.

Electromagnetic energy scale (LAr) The uncertainty on the electromagnetic energy scale of the Liquid Argon calorimeter amounts to $1.5 \%$ after the in-situ $Z \rightarrow e e$ calibration as described in Section 2.5.1. This uncertainty is applied to particles not included in the single particle analysis, e.g. photons from $\pi^{0}$ decays and electrons.

Electromagnetic energy scale (Tile) For the hadronic calorimeter, the electromagnetic scale uncertainty is estimated considering the ratio of the electromagnetic scale response of minimum ionizing low-energy muons in test-beam data and in Monte Carlo as described in Section 2.5.2. This uncertainty amounts to $3 \%$, and it is applied to all particles interacting in the Tile calorimeter not included in the single particle analysis, e.g. hadrons with momentum above $20 \mathrm{GeV}$.

The systematic effects due to the presence of additional material in front of the calorimeters are only constrained for particles included in in-situ measurements (hadrons with momenta $p<20 \mathrm{GeV}$, electrons and photons). The remaining uncertainty due to additional material is estimated using dedicated Monte Carlo simulation samples detailed in 5.2.3 where the detector material is systematically varied within the current uncertainty on the detector geometry knowledge.

\section{Evaluation of the calorimeter jet energy scale uncertainty}

The starting point for the evaluation of the calorimeter jet energy scale uncertainty through single particle studies is a series of pseudo experiments. In each pseudo experiment the energy deposits for each single particle included in a jet are varied within statistical and systematic uncertainties depending on the nature and momentum of the particle as mentioned above. Each pseudo experiment contributes to a spread of possible jet energies, where the energy deposits that constitute every jet have been varied within the uncertainties estimated as described in the previous section. The mean relative energy shift, defined as the ratio between the jet energy estimated from the variations on the energy deposits and that obtained from the central value of each energy deposit, is estimated for each pseudo experiment as shown in Figure 5.2 (a). The overall uncertainty from single particle studies is derived from a Gaussian fit of the distribution of the mean energy shifts for all pseudoexperiments, for each $p_{T}^{\text {jet }}$ and $\eta$ bin, as shown in Figure 5.2 (b).

Figure 5.3 shows the contribution to the JES uncertainty from the calorimeter response, derived from single particle studies. In the pseudorapidity range $0 \leq|\eta|<0.8$ the shift of the 


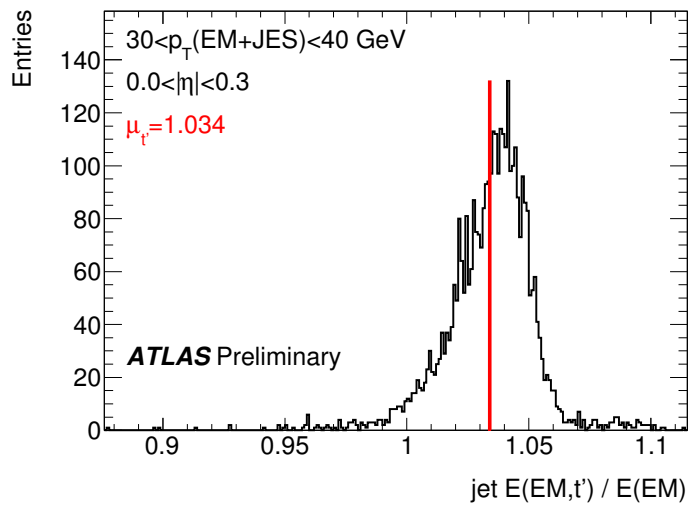

(a)

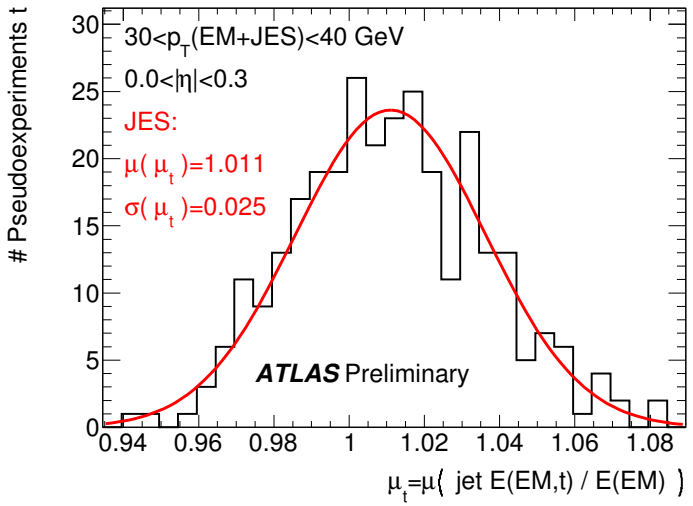

(b)

Figure 5.2.:Figure (a): example of a single pseudo experiment where the energies deposited from particles within a jet $\left(30<p_{T}<40 \mathrm{GeV}\right.$, anti- $\left.k_{t} R=0.6\right)$ have been varied within their uncertainties, causing a shift in the jet energy scale (red line). Figure (b): distribution of the expected shift and uncertainty on the jet energy scale from all pseudo-experiments as in (a). Figure from [49].

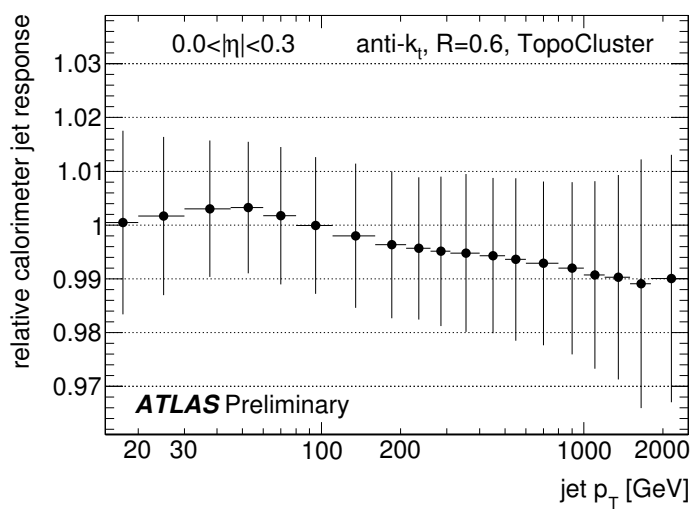

(a)

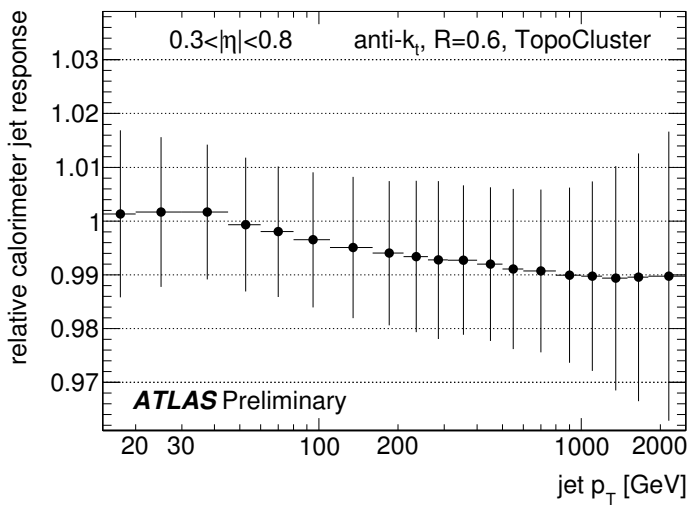

(b)

Figure 5.3.:Expected shift (as the mean of the jet response distribution over all pseudoexperiments, black dots) and total uncertainty (as the RMS of the jet response distribution over all pseudoexperiments, error bars) on the relative calorimeter jet response for anti- $k_{t} R=0.6$ jets in the range $0 \leq|\eta|<0.3$ (left) and $0.3 \leq|\eta|<0.8$ (right) as function of the jet transverse momentum [128].

relative jet energy scale (also called bias in the following) is up to $\approx 1 \%$, and the uncertainty on the shift is from 1 to $3 \%$. The total envelope (the shift added linearly to the uncertainty) of about $1.5-4 \%$ depending on the jet transverse momentum is taken as the relative JES calorimeter uncertainty. 


\section{Correlations in the calorimeter uncertainty sources}

The pseudo experiment approach adopted to derive the calorimeter uncertainty also provides the tools to derive the correlation matrix, shown for anti- $k_{t} R=0.6$ in Figure 5.4. Neighboring bins where particles of the same momentum enter the jets have almost full correlation, but decorrelations are present between low and high $p_{T}$ bins.

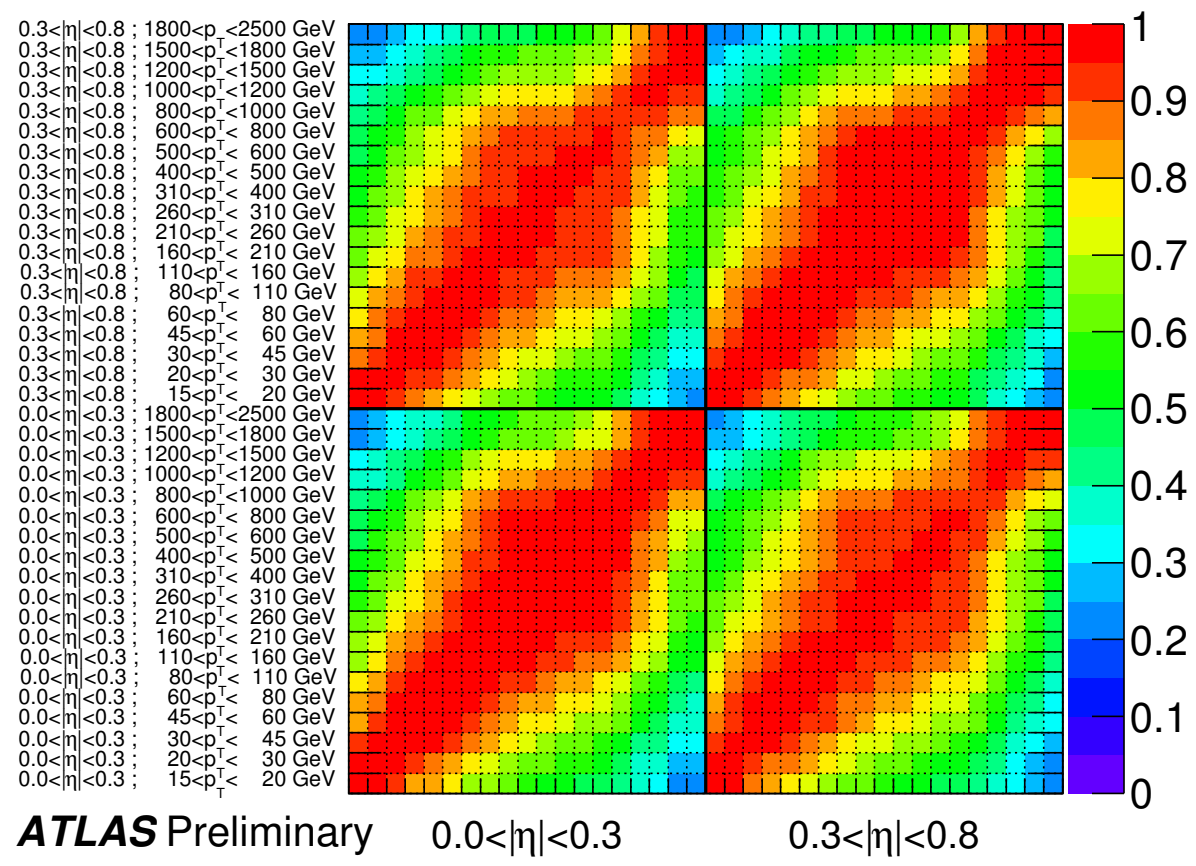

Figure 5.4.:Correlation coefficient of the calorimeter uncertainty source for anti- $k_{t} R=0.6$ jets, where the color scale gives the size of the bin-to-bin correlation and the $\mathrm{x}$ axis labels reflect those of the y axis. Similar results are found for anti- $k_{t} R=0.4$ jets. Figure from [128].

\subsubsection{Uncertainties due to the detector simulation}

The effects related to the detector and calorimeter simulation and to the experimental conditions that could contribute to the jet energy scale uncertainty are listed below, and described in more detail in this Section.

1. The electronic noise in data could differ from the noise description used in the Monte Carlo simulation, and these differences propagated to the Monte Carlo based calibration constants. The effect on the jet energy scale is accounted for in the topocluster noise thresholds uncertainty.

2. The jet energy scale is affected by an increased amount of inactive material for particles not included in in-situ measurements. The energy deposited by particles in the material 
that is not included in the simulation is neither accounted for in the present calibration procedure and it is only partially included in the uncertainty on the calorimeter response. The full effect of additional dead material on the jet energy scale is estimated within the additional detector material uncertainty.

\section{Calorimeter cell noise thresholds}

As described in Section 3.1.3, topoclusters constitute the input to the jet reconstruction. They are constructed based on the signal-to-noise ratio of calorimeter cells, where the noise refers to the RMS of the measured cell energy distribution in events with no energy depositions from collision events from dedicated runs (e.g. random triggers or empty bunches). In the case of data, the noise can change over time ${ }^{5}$, while the noise RMS used in the simulation are fixed at the time of the production of the simulated data. This, and the underlying assumptions used for the noise modelling in the Monte Carlo simulation, can lead to differences in the cluster shapes and to the presence of fake clusters, which in turn affect the jet reconstruction and calibration.

Noise modeling enters the Monte Carlo simulation in the following ways, as schematically pictured in Figure 5.5:

1. the simulated electronic noise (dark red area) is superimposed to the signal of particles passing through active material of the calorimeters (blue curve);

2. cell-level constants for the RMS of the energy distribution from random events are stored in the database and applied during the topocluster reconstruction (light red curve and magenta lines).

The timescale required for the final JES uncertainty estimate did not allow the production of dedicated Monte Carlo samples where the noise injected in the Monte Carlo simulation was modified for selected cells. Instead, the topocluster thresholds were varied according to noise in data, using noise constants taken from a cell noise table of a selected data run in the reconstruction of Monte Carlo jets. This means that the actual energy and noise simulated in the MC are left unchanged, but the values of the thresholds used to include a given calorimeter cell in a topocluster are based on the noise RMS for the cell as measured in data. Pictorially, this implies that the light red curve in Figure 5.5 is substituted with the noise distribution in data, since the threshold constants are obtained from the parameterization of the data-derived noise distribution.

\footnotetext{
${ }^{5}$ Time-dependent noise changes for single cells in data are accounted for using periodic measurements of the cell noise.
} 


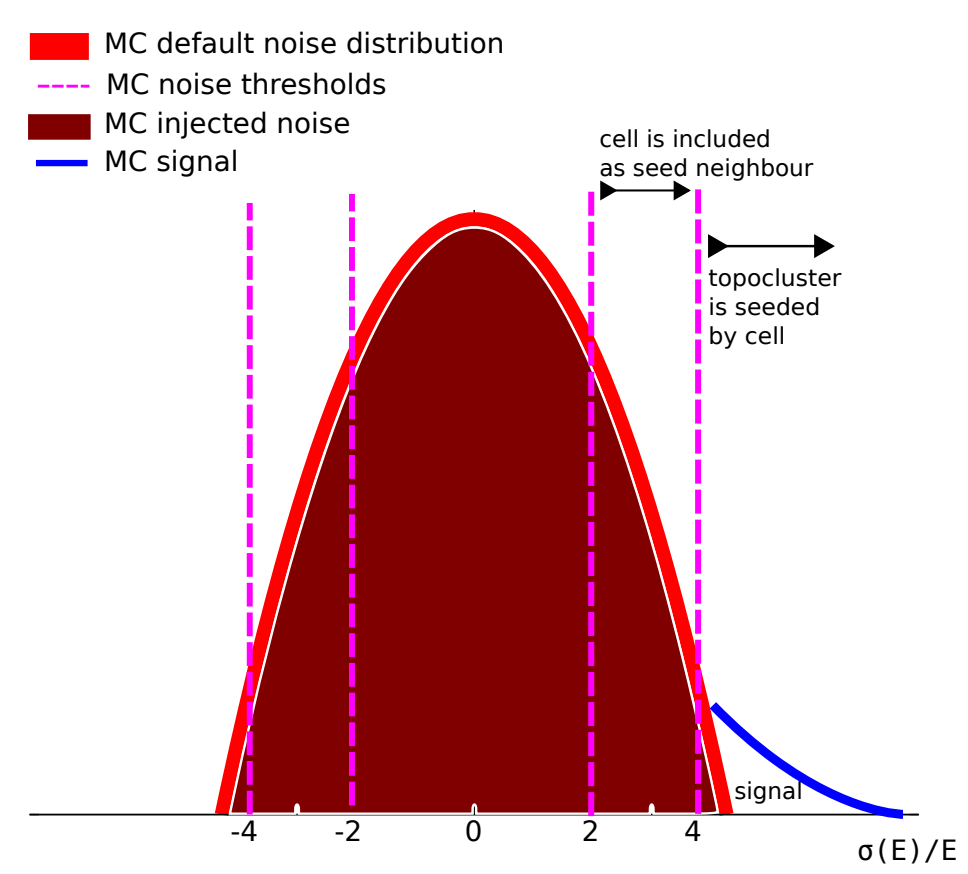

Figure 5.5.:Schematic representation of the cell noise distribution and topocluster threshold constants in Monte Carlo.

The noise recorded in data could be increased with respect to what is assumed by the constants in the database for the topocluster thresholds, as shown in Figure 5.6 (a), and lead to the creation of fake topoclusters or the inclusion of noisy cells in the topoclusters. The opposite situation in Figure 5.6 (b) could happen if the cell has a narrower noise distribution with respect to what predicted by the Monte Carlo. However, the cell noise constants extracted from data only contain an average snapshot of the cells that are affected by non-nominal noise conditions at any point in time.

To fully bracket both situations of Figure 5.6 and take into account possible variations in the cell noise extracted from data with time, the case in which all topocluster thresholds are shifted upwards and downwards is used as a cross check. Different Monte Carlo samples have been reconstructed with signal-to-noise thresholds for all topocluster seed and neighbor cells modified to be $10 \%$ and $7 \%$ higher and lower than the respective nominal values of 4 and 2 .

The less conservative value of $7 \%$ is still found to fully bracket the shift in the jet response to using the noise RMS from data in the low $p_{\mathrm{T}}^{\text {jet }}$ region where the effect of varying the cell noise thresholds is larger. Raising and lowering the cell thresholds by $7 \%$ leads to a roughly symmetric effect on the jet response. This allows the use of the calorimeter cell noise thresholds derived from data as a symmetric uncertainty that covers both the case when more and less noise is present in data with respect to the simulation, and complements the estimate of the 


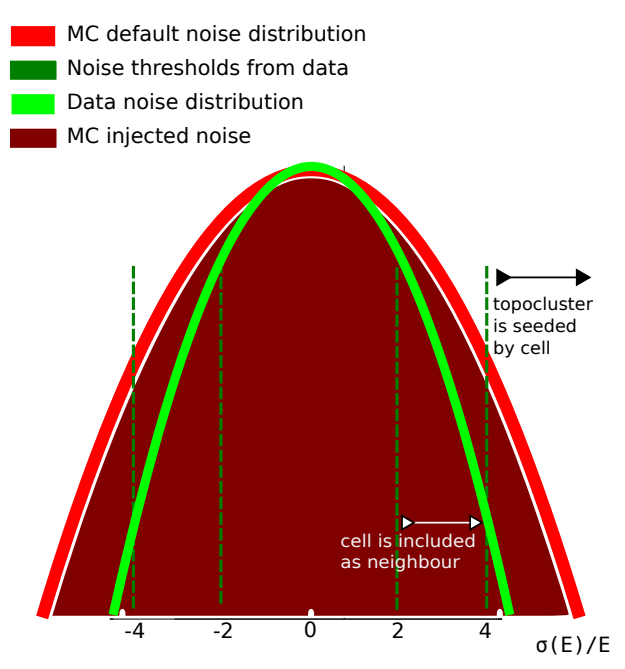

(a)

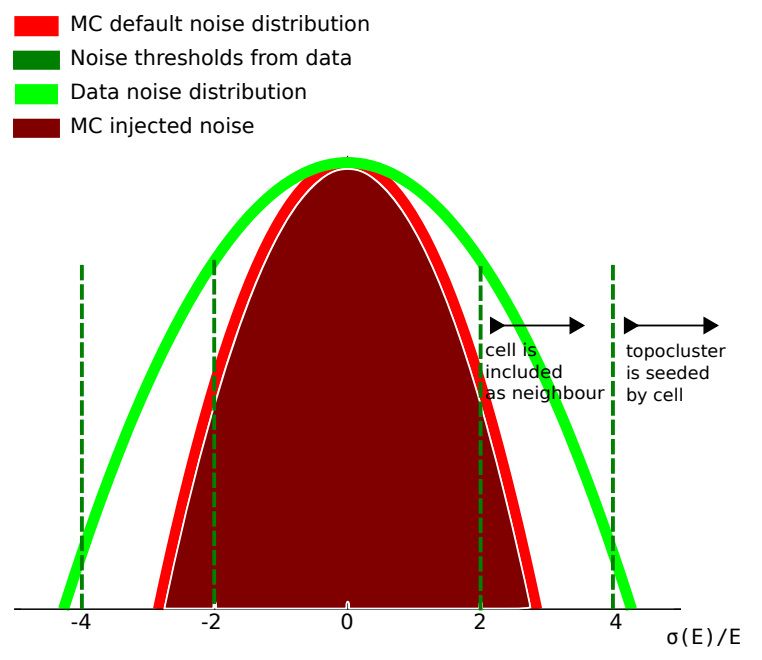

(b)

Figure 5.6.:Schematic representation of the cell noise distribution in Monte Carlo when the noise constants are taken from a cell noise table of a selected data run in the case of increased (a) and decreased (b) noise in the cell in data.

calorimeter cell noise threshold uncertainty where data-derived thresholds are applied to Monte Carlo simulation.

The response of jets reconstructed with the data-derived noise thresholds is compared to the response of the jets reconstructed in exactly the same sample using the default MC noise thresholds. The maximal observed change between the two samples is used to estimate the uncertainty on the jet energy measurement due to the calorimeter cell noise modelling. As shown for two example $p_{\mathrm{T}}^{\text {jet }}$ bins in Figure 5.7, the uncertainty is found to be below $2 \%$ for the whole pseudorapidity range, and negligible for jets with transverse momenta above $45 \mathrm{GeV}$. The uncertainties assigned to jets with transverse momenta below $45 \mathrm{GeV}$ are:

- $1 \%$ and $2 \%$ for $20 \mathrm{GeV} \leq p_{\mathrm{T}}^{\text {jet }}<30 \mathrm{GeV}$ for anti- $k_{t}$ with distance parameters $R=0.4$ and $R=0.6$ jets respectively,

- $1 \%$ for $30 \mathrm{GeV} \leq p_{\mathrm{T}}^{\text {jet }}<45 \mathrm{GeV}$.

\section{Additional detector material}

The jet energy scale is affected by possible deviations in the material description: its calibration has been derived to correct for the energy lost under the assumption of the geometry simulated in the nominal Monte Carlo sample. Simulated detector geometries that include systematic variations to the material budget have been designed using test-beam measurements [72], in 


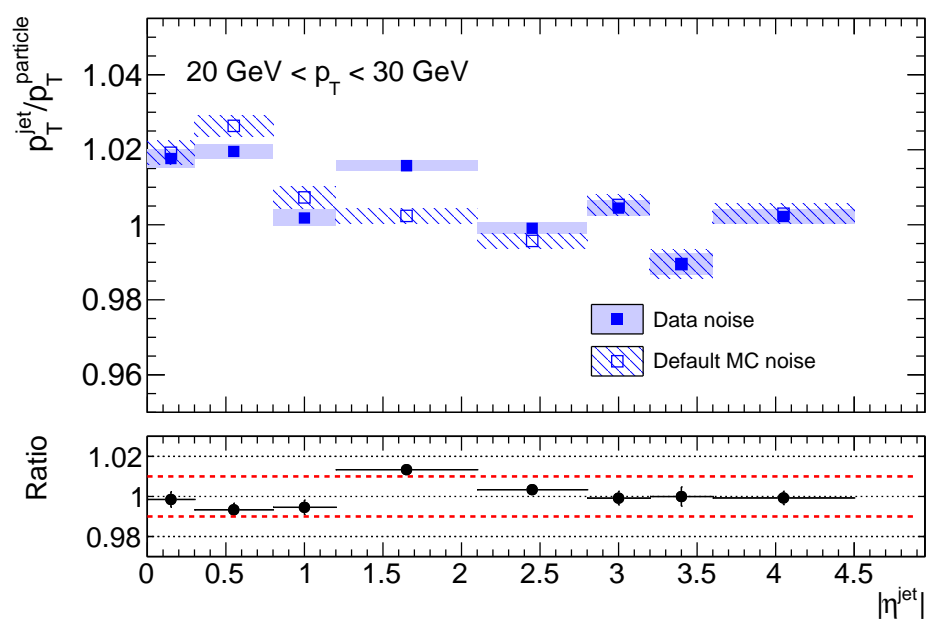

(a)

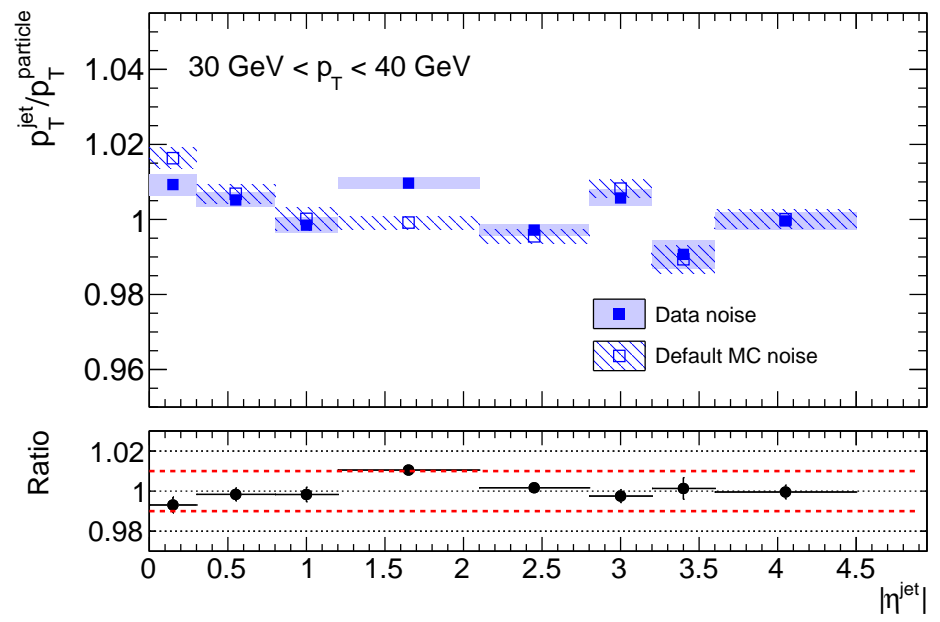

(b)

Figure 5.7.:Jet response as a function of pseudorapidity for simulated QCD anti- $k_{t} R=0.6$ jets reconstructed with the default (filled area and full squares) and data-derived (shaded area and empty squares) noise threshold constants in two different $p_{\mathrm{T}}^{\text {jet }}$ bins.

addition to studies in $900 \mathrm{GeV}$ and $7 \mathrm{TeV}$ data [96,97,130,131]. Specific Monte Carlo samples have been reconstructed using these distorted geometries.

In the case of uncertainties derived with in-situ techniques, such as those coming from the $E / p$ measurements detailed in Section 5.2.2, most of the effects on the jet response due to additional dead material are already taken into account because the measurement is performed directly on the ATLAS detector. However, the quality criteria on the track selection for the $E / p$ measurement effectively only allow particles that have not interacted in the inner detector to be 
included in the measurement. Therefore the effect of dead material in the inner detector needs to be taken into account for particles in the momentum range of the $E / p$ measurement. This is achieved using a specific Monte Carlo sample where the material budget is systematically varied by adding $5 \%$ of material to the existing inner detector services. The uncertainty derived from the comparison of the distorted material response to the nominal response is then scaled by the fraction of particles within the $E / p$ momentum range. This uncertainty is shown in Figure 5.8.

Electrons, photons and hadrons with momenta $p>20 \mathrm{GeV}$ are not included in the $E / p$ measurements and therefore there is no in-situ estimate on the effect of any additional material in front of the calorimeters. This uncertainty is estimated using a dedicated Monte Carlo simulation sample where the overall detector material is systematically varied within the current uncertainties of the detector geometry knowledge. The overall changes in the detector geometry include:

- the $5 \%$ increase of inner detector material mentioned above;

- an extra 0.1 radiation length $\left(X_{0}\right)$ placed in the cryostat in front of the barrel of the electromagnetic calorimeter $(|\eta|<1.5)$;

- an extra $0.05 X_{0}$ placed between the presampler and the first layer of the electromagnetic calorimeter;

- an extra $0.1 X_{0}$ placed in the cryostat after the barrel of the electromagnetic calorimeter;

- extra material is also present in the barrel-endcap transition region in the electromagnetic calorimeter $(1.37<|\eta|<1.52)$, corresponding to 1.5 times the amount of nominal material.

The uncertainty contribution due to the overall additional detector material is estimated by comparing the calibrated EM+JES jet response in the Monte Carlo sample with the distorted geometry with the nominal jet response (see Figure 5.8), and scaled by the average fraction of electrons, photons and high transverse momentum hadrons within a jet as a function of $p_{T}$.

In the endcap and forward calorimeter regions, a proper assessment of the detector material in front of the calorimeters is difficult, and no estimate of the dead material uncertainty has been attempted. The dead material uncertainty contribution in the endcap region is included in the JES uncertainty from the measurement of the relative di-jet $p_{\mathrm{T}}^{\mathrm{jet}}$ imbalance between central and endcap jets in data and Monte Carlo simulation that is described in Section 5.2.5. 


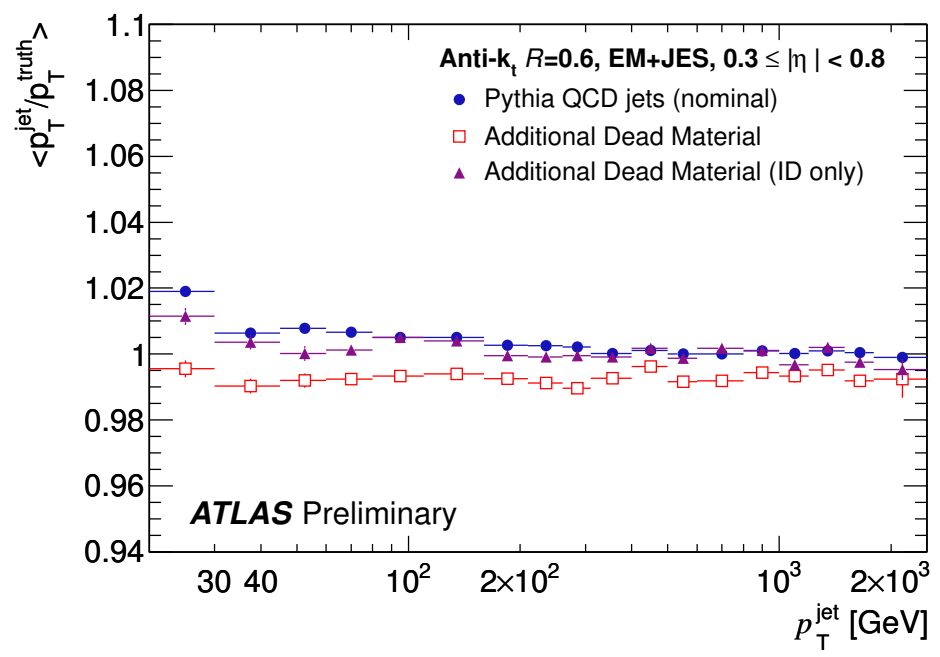

Figure 5.8.:Simulated $p_{\mathrm{T}}^{\text {jet }}$ response as a function of $p_{\mathrm{T}}^{\text {jet }}$ in the central region $(0.3<|\eta| \leq 0.8)$ in the case of additional dead material in the inner detector (full triangles) and in both the inner detector and the calorimeters (open squares). The response within the nominal Monte Carlo sample is shown for comparison (full circles).

\subsubsection{Uncertainties due to the event modelling in the Monte Carlo generators}

The contributions to the JES uncertainty due to the modelling of the fragmentation, underlying event and to the parameters of the Monte Carlo event generator are obtained using the Perugia2010 Pythia tune and AlPgen + Herwig + Jimmy Monte Carlo samples introduced in Section 4.2.1. By comparing the nominal PyтHIA Monte Carlo sample to that simulated with the PyтніA Perugia2010 tune, the effects of soft physics modelling (e.g. underlying event) are tested. The Perugia2010 tune provides in particular a better description of the internal jet structure recently measured in ATLAS [132]. The ALPGEN + HERWIG + JIMMY simulation sample differs with respect to the nominal PYTHIA sample in many respects, namely:

- the AlPGEN generator differs from PyThia in the leading order matrix element calculation of multiple partons (legs) in the final state: $2 \rightarrow 2$ to $2 \rightarrow 5$ legs are simulated for ALPGEN compared to $2 \rightarrow 2$ only for PYTHIA

- the matrix element matching to the parton shower is done with the MLM algorithm in the ALPGEN sample,

- the CTEQ6L1 parton distribution function used for ALPGEN, compared to the MRST LO* set used for PYTHIA, 
- the angular-ordered parton shower in HERwIG, compared with the $p_{T}$ ordered shower in PYTHIA,

- the cluster model for fragmentation implemented in HERWIG, compared to the PYTHIA string model,

- the underlying event as implemented in JimmY, compared to the PYTHIA model.

The AlPGEn Monte Carlo uses different models for all phases of the event generation and therefore gives a reasonable estimate of the systematic variations. However, the possible compensation of effects that shift the jet response in opposite directions cannot be excluded.

Figure 5.9 shows the calibrated jet kinematic response for the two Monte Carlo generators and tunes used to estimate the effect of Monte Carlo theoretical model on the jet energy scale uncertainty, together with the kinematic response for the nominal sample shown for comparison, for the evaluation of the JES uncertainty for the full 2010 dataset.

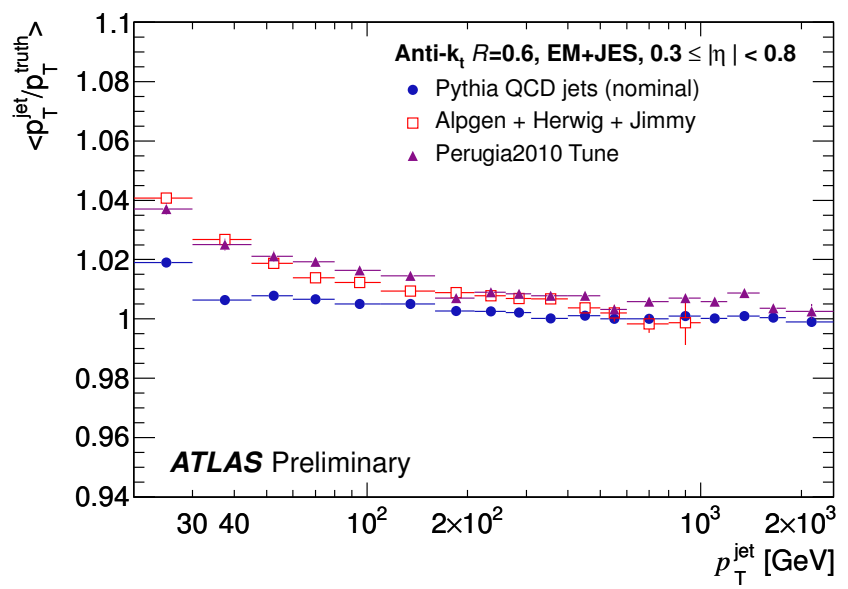

Figure 5.9.:Simulated $p_{\mathrm{T}}^{\text {jet }}$ response as a function of $p_{\mathrm{T}}^{\text {jet }}$ in the central region $(0.3<|\eta| \leq 0.8)$ for Alpgen + Herwig + Jimmy (open squares) and Pythia with the Perugia2010 tune (full triangles). The response of the nominal Monte Carlo sample is shown for comparison (full circles).

\subsubsection{Uncertainties due to the relative calibration (endcap/forward regions)}

The JES uncertainty, determined in the central detector region using the single particle response and systematic variations of the Monte Carlo simulations, is transferred to the forward regions by exploiting the transverse momentum balance of a central and a forward jet in events with 
dijet topologies as detailed in Section 4.2.1. This choice is motivated by the better knowledge of the material budget for the barrel region with respect to the endcap region.

The jet energy scale uncertainty for jets with $|\eta|>0.8$ is estimated as follows:

- the total JES uncertainty in the central region $0.3<|\eta|<0.8$ is kept as a baseline. This is the largest fully instrumented $|\eta|$ region considered where the combined test-beam results used to estimate the calorimeter uncertainty are available for the entire pseudorapidity range.

- the uncertainty from the relative intercalibration, taken as the RMS deviation of the MC predictions from the data, is added in quadrature to the baseline uncertainty.

The intercalibration uncertainty is measured in bins of the average $p_{\mathrm{T}}$ of the two leading jets, labelled $p_{\mathrm{T}}^{\text {avg }}$. Due to momentum balance, this quantity is on average similar to the average transverse momenta of any of the two jets. The measurements are performed for transverse momenta in the range $20 \leq p_{\mathrm{T}}^{\text {avg }}<110 \mathrm{GeV}$. The uncertainty for jets with $p_{\mathrm{T}}>100 \mathrm{GeV}$ is taken as the uncertainty of the last available $p_{\mathrm{T}}$-bin. This is justified by the decrease of the intercalibration uncertainty with $p_{\mathrm{T}}$, but cannot completely exclude the presence of calorimeter non linearities for jet energies above those used for the intercalibration.

The uncertainties are evaluated separately for jets reconstructed with distance parameters $R=0.4$ and $R=0.6$, and are in general found to be slightly larger for $R=0.4$.
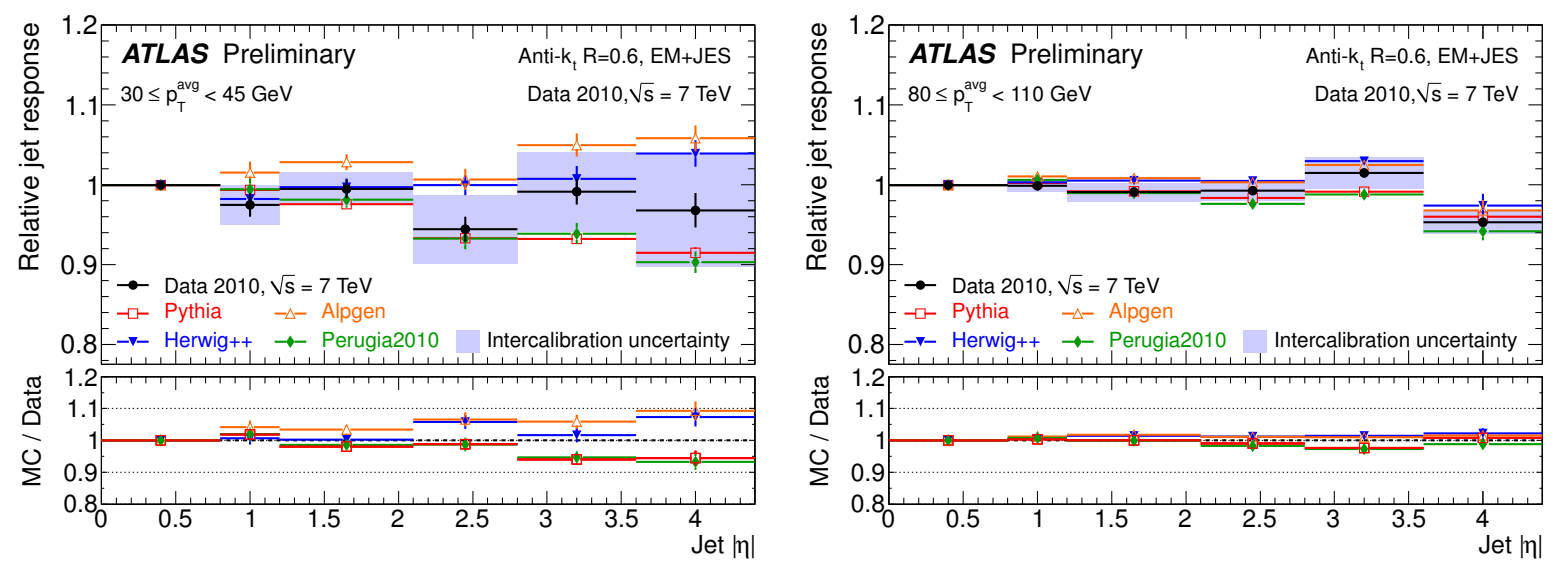

Figure 5.10.:Jet $p_{\mathrm{T}}$ response measured relative to a central reference jet in data and various MC generator samples for jet $p_{\mathrm{T}}$ in the ranges $30-45 \mathrm{GeV}$ (left) and $80-110 \mathrm{GeV}$ (right). The resulting uncertainty component is shown as a shaded band around the data points. Figure from [111].

Figure 5.10 shows the relative jet response, and the associated intercalibration uncertainty calculated as detailed above, as a function of jet $|\eta|$ for two representative $p_{\mathrm{T}}^{\text {avg }}$-bins. 


\subsection{Uncertainty due to multiple interactions}

Particles produced by multiple soft proton-proton interactions in the same bunch crossing additional to the event of interest (in-time pile-up) can produce additional energy deposits that are reconstructed within the jet. As briefly described in Section 3.2.1, and fully detailed in Ref. [57], an average offset correction is applied to account for the average increase of the jet energy due to pile-up. This correction is parameterised as a function of the number of measured primary vertices $N_{\mathrm{PV}}$.

The estimate of the remaining uncertainty on the jet energy scale after applying the pile-up correction is based on the studies described in Section 5 of Ref. [57]. The contributions to the uncertainty are estimated from studies that account for:

- the variation of the average offset-corrected calorimeter jet energy for calorimeter jets matched to track-jets as a function of the number of primary vertices,

- the effects of the variation of the trigger selection on the measured tower energy distribution that is input to the offset correction,

- the mapping of the tower-based offset correction to jets built from topoclusters using the average number of towers,

- the non-closure of the tower-based offset correction as evaluated by the dependence of the corrected calorimeter jet energy for calorimeter jets matched to track-jets.

The uncertainty on the jet energy scale is conservatively estimated by adding all uncertainties above in quadrature. Since the track-jet method can be used only up to $|\eta|<1.9$ due to the limited coverage of the tracking detector, the di-jet balance method detailed in Section 4.2.1 has been used to estimate the uncertainty for $|\eta|>1.9$. In this case, the relative jet response in events with only one reconstructed vertex was compared with the response measured in events with several reconstructed vertices. The dijet balance method yields uncertainties similar to those detailed above also in the case of $|\eta|>1.9$.

The offset correction and its uncertainty are derived as a function of the number of reconstructed vertices. This allows the correction and its uncertainty to be valid also for data periods where the number of reconstructed primary vertices is higher than the period where the correction is derived.

Figure 5.11 shows the relative uncertainty due to pile-up in the case of two measured primary vertices for jets with anti- $k_{t} R=0.6$. In this case, the uncertainty due to pile-up for central jets 


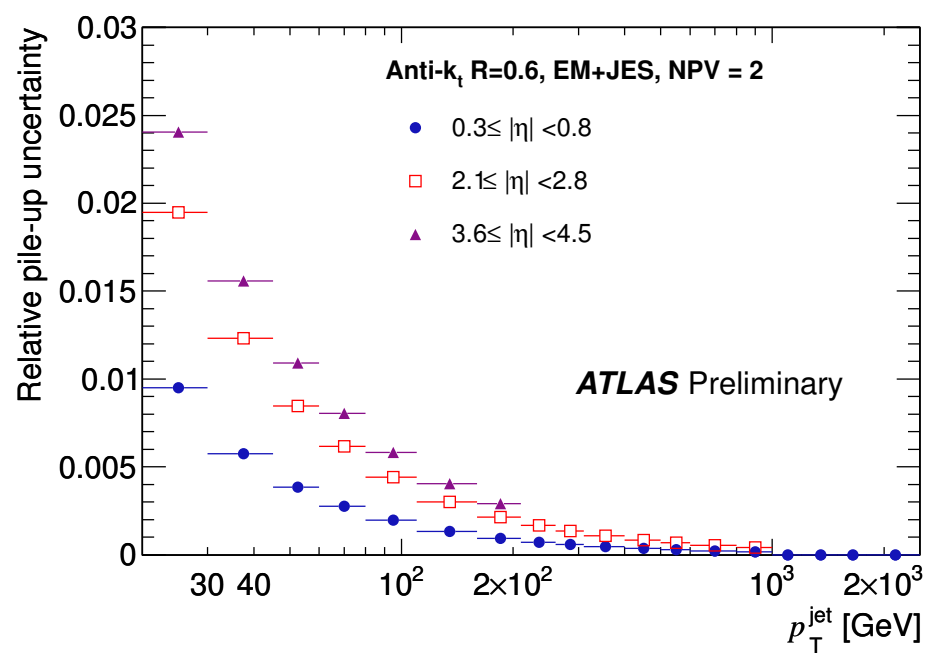

Figure 5.11.:Relative pile-up uncertainty for anti- $k_{t}$ jets with $R=0.6$ in the case of two measured primary vertices, $N_{\mathrm{PV}}=2$, for central $(0.3<|\eta| \leq 0.8$, full circles $)$, endcap $(2.1<|\eta| \leq 2.8$, open squares) and forward $\left(3.6<|\eta| \leq 4.5\right.$, full triangles) jets as a function of jet $p_{\mathrm{T}}$.

\begin{tabular}{|lc|c|c|c|c|c|c|}
\hline & & $N_{\mathrm{PV}}=2$ & $N_{\mathrm{PV}}=3$ & $N_{\mathrm{PV}}=4$ & $N_{\mathrm{PV}}=5$ & $N_{\mathrm{PV}}=6$ & $N_{\mathrm{PV}}=7$ \\
\hline \multirow{2}{*}{$0.3 \leq|\eta|<0.8$} & $p_{T}=20 \mathrm{GeV}$ & 0.010 & 0.019 & 0.030 & 0.040 & 0.050 & 0.060 \\
& $p_{T}=200 \mathrm{GeV}$ & 0.001 & 0.002 & 0.003 & 0.004 & 0.005 & 0.006 \\
\hline \multirow{2}{*}{$2.1 \leq|\eta|<2.8$} & $p_{T}=20 \mathrm{GeV}$ & 0.019 & 0.04 & 0.062 & 0.085 & 0.11 & 0.13 \\
& $p_{T}=200 \mathrm{GeV}$ & 0.002 & 0.004 & 0.007 & 0.009 & 0.012 & 0.014 \\
\hline \multirow{2}{*}{$3.6 \leq|\eta|<4.5$} & $p_{T}=20 \mathrm{GeV}$ & 0.024 & 0.050 & 0.079 & 0.11 & 0.14 & 0.16 \\
& $p_{T}=200 \mathrm{GeV}$ & 0.003 & 0.006 & 0.010 & 0.013 & 0.016 & 0.020 \\
\hline
\end{tabular}

Table 5.2.:Pile-up uncertainty for anti- $k_{t} R=0.6$ jets.

with $p_{T}=20 \mathrm{GeV}$ and pseudorapidity $|\eta| \leq 0.8$ is about $1 \%$, while it amounts to about $2 \%$ for jets with pseudorapidity $2.1<|\eta|<2.8$ and to less than $2.5 \%$ for all jets with $|\eta| \leq 4.5$. In the case of more primary vertices, the pile-up uncertainty is summarised in Table 5.2 for jets of $p_{T}=20$ and $200 \mathrm{GeV}$ for the three example $\eta$ regions $(0.3 \leq|\eta|<0.8,2.1 \leq|\eta|<2.8$ and $3.6 \leq|\eta|<4.5$ ). Jets with anti- $k_{t} R=0.4$ show a similar trend, albeit with slightly smaller uncertainties due to the reduced jet area.

The pile-up uncertainty needs to be added separately to the estimate of the total jet energy scale uncertainty detailed in Section 5.4.

The effect of additional proton-proton interactions from different bunch crossings that can be caused by trains of consecutive bunches (out-of-time pile-up) has been studied separately. The effect of out-of-time pile-up on jet reconstruction has been studied matching calorimeter jets 
and track jets (unaffected by the integration time of the calorimeters) and has been found to be negligible in the 2010 data-set considered for the measurement in this thesis.

\subsection{Combination of JES uncertainty sources}

The total jet energy scale uncertainty has been derived by considering all the individual contributions described in Section 5.2. In the central region $(|\eta|<0.8)$, the estimate proceeds as follows:

1. For each $p_{\mathrm{T}}^{\text {jet }}$ and $\eta$ bin, the uncertainty due to the calibration procedure is calculated as described in Section 5.2.1 for both jet energy and $p_{\mathrm{T}}$ response. For each bin, the maximum deviation from unity between the energy and $p_{\mathrm{T}}$ response is taken as the final non-closure uncertainty.

2. The calorimeter response uncertainty is estimated as a function of jet $\eta$ and $p_{T}$ from the propagation of single particle uncertainties to the jets, as detailed in Section 5.2.2.

3. Sources of uncertainties estimated using Monte Carlo samples with a systematic variation are accounted as follows:

a) the response in the test sample $\mathcal{R}_{\text {var }}$ and the response in the nominal sample $\mathcal{R}_{\text {nom }}$ is considered as a starting point for the estimate of the JES uncertainty. The deviation of this ratio from unity is defined as:

$$
\Delta_{\mathrm{JES}}\left(p_{T}^{\mathrm{jet}}, \eta\right)=\left|1-\frac{\mathcal{R}_{\mathrm{var}}\left(p_{T}^{\mathrm{jet}}, \eta\right)}{\mathcal{R}_{\mathrm{nom}}\left(p_{T}^{\mathrm{jet}}, \eta\right)}\right| .
$$

This deviation is calculated from both the energy and $p_{T}$ response, leading to $\Delta_{\mathrm{JES}}^{\mathrm{E}}\left(p_{T}^{\mathrm{jet}}, \eta\right)$ for the deviation in the energy response, and to $\Delta_{\mathrm{JES}}^{\mathrm{p}_{\mathrm{T}}}\left(p_{T}^{\mathrm{jet}}, \eta\right)$ for the deviation in the transverse momentum response.

b) The largest $\Delta_{\mathrm{JES}}$ in each bin derived from the jet energy $(E)$ or transverse momentum $\left(p_{T}\right)$ response is considered as the contribution to the final JES systematic uncertainty due to the specific systematic effect:

$$
\Delta_{\mathrm{JES}}\left(p_{T}^{\mathrm{jet}},|\eta|\right)=\max \left(\Delta_{\mathrm{JES}}^{\mathrm{E}}\left(p_{T}^{\mathrm{jet}}, \eta\right), \Delta_{\mathrm{JES}}^{\mathrm{p}_{\mathrm{T}}}\left(p_{T}^{\mathrm{jet}}, \eta\right)\right)
$$


4. The estimate of the uncertainty contributions due to additional material in the inner detector and overall additional inactive material is estimated as described in point 3 . above. These uncertainties are then scaled by the average fraction of particles forming the jet that are within $p<20 \mathrm{GeV}$ (for the inner detector distorted geometry) and by the average fraction of particles outside the $E / p$ in-situ measurements (for the overall distorted geometry), as mentioned in Section 5.2.3.

For each $p_{\mathrm{T}}^{\text {jet }}, \eta$ bin, the contributions from the calibration non-closure, calorimeter response, Monte Carlo variations and additional inactive material listed above are added in quadrature.

For pseudorapidities beyond $|\eta|>0.8$, the $\eta$ intercalibration contribution is estimated for each pseudorapidity bin in the endcap region as detailed in Section 5.2.5. The intercalibration contribution is added in quadrature to the total JES uncertainty determined in the $0.3 \leq|\eta|<0.8$ region to estimate the JES uncertainty for jets with $|\eta|>0.8$, with the exception of the nonclosure term that is retained for the specific $\eta$ region. For low jet $p_{T}$, this choice leads to partially double count the contribution from the dead material uncertainty, but it is considered as a conservative estimate in a region where it is difficult to estimate the accuracy of the material description.

The contribution to the uncertainty due to additional proton-proton interactions described in Section 5.3 needs to be added separately, depending on the number of primary vertices in the event. In the following, only the uncertainty in the case of a single proton-proton interaction is shown in detail.

\subsection{Results}

Figures 5.12, 5.13 and 5.14 show the final fractional jet energy scale systematic uncertainty and its individual contributions as a function of jet $p_{\mathrm{T}}^{\text {jet }}$ for three selected $\eta$ regions. Plots for all pseudorapidity bins and both jet distance parameters can be found in Appendix C.

The fractional JES uncertainty in the central region amounts to 2 to $4 \%$ for $p_{\mathrm{T}}^{\text {jet }}<60 \mathrm{GeV}$, and it is between 2 and $2.5 \%$ for $60 \mathrm{GeV} \leq p_{\mathrm{T}}^{\text {jet }}<800 \mathrm{GeV}$. For jets with $p_{\mathrm{T}}^{\text {jet }}>800 \mathrm{GeV}$, the uncertainty ranges from 2.5 to $4 \%$, due to the larger uncertainties for particles with momentum beyond $400 \mathrm{GeV}$ comprised in these jets. The uncertainty amounts to up to $7 \%$ and $3 \%$, respectively, for $p_{\mathrm{T}}^{\text {jet }}<60 \mathrm{GeV}$ and $p_{\mathrm{T}}^{\text {jet }}>60 \mathrm{GeV}$ in the endcap region, where the central uncertainty is taken as a baseline and the uncertainty due to the relative calibration is added. In the forward region, a $13 \%$ uncertainty is present for $p_{\mathrm{T}}^{\text {jet }}<60 \mathrm{GeV}$ : the increase in the 


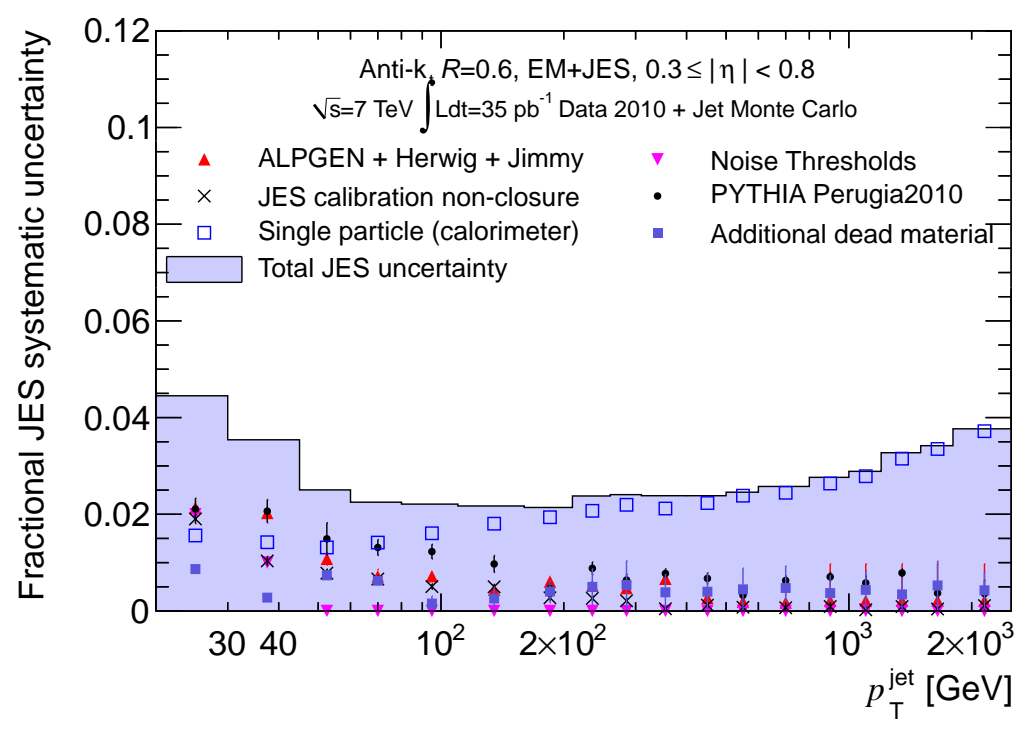

Figure 5.12.:Fractional jet energy scale systematic uncertainty as a function of $p_{\mathrm{T}}^{\text {jet }}$ for jets in the pseudorapidity region $0.3 \leq|\eta|<0.8$ in the calorimeter barrel. The total uncertainty is shown as the solid light blue area. The individual sources are also shown, with uncertainties from the fitting procedure if applicable.

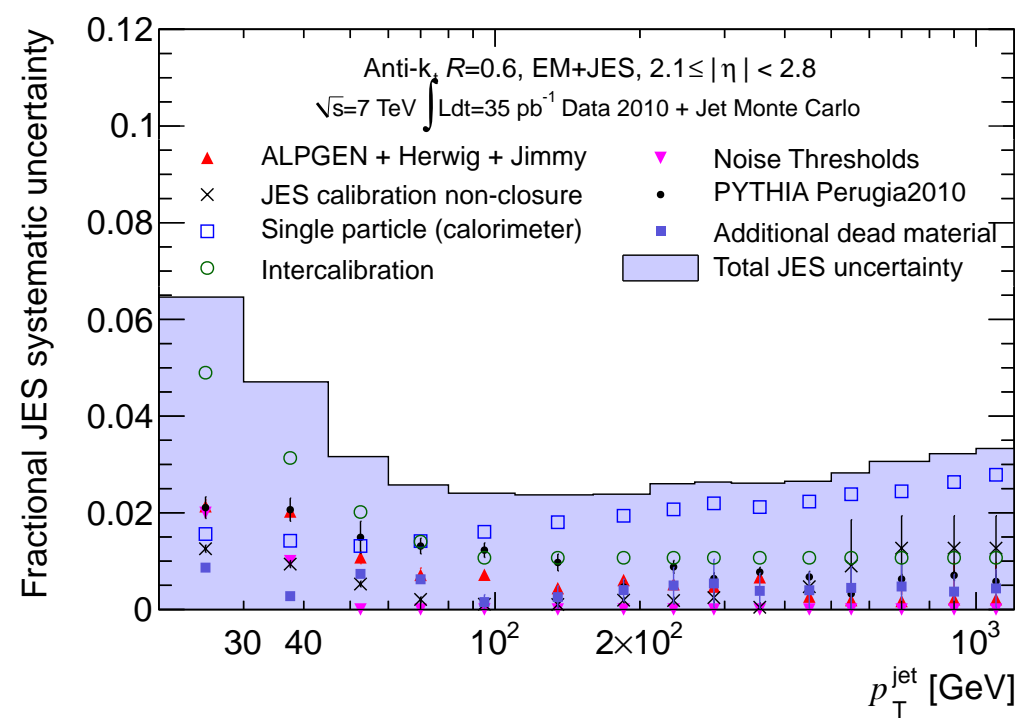

Figure 5.13.:Fractional jet energy scale systematic uncertainty as a function of $p_{\mathrm{T}}^{\text {jet }}$ for jets in the pseudorapidity region $2.1 \leq|\eta|<2.8$. The JES uncertainty in this endcap region is extrapolated from the barrel uncertainty, with the uncertainty contribution from the $\eta$ intercalibration between central and endcap jets in data and Monte Carlo added in quadrature. The total uncertainty is shown as the solid light blue area. The individual sources are also shown, with uncertainties from the fitting procedure if applicable. 


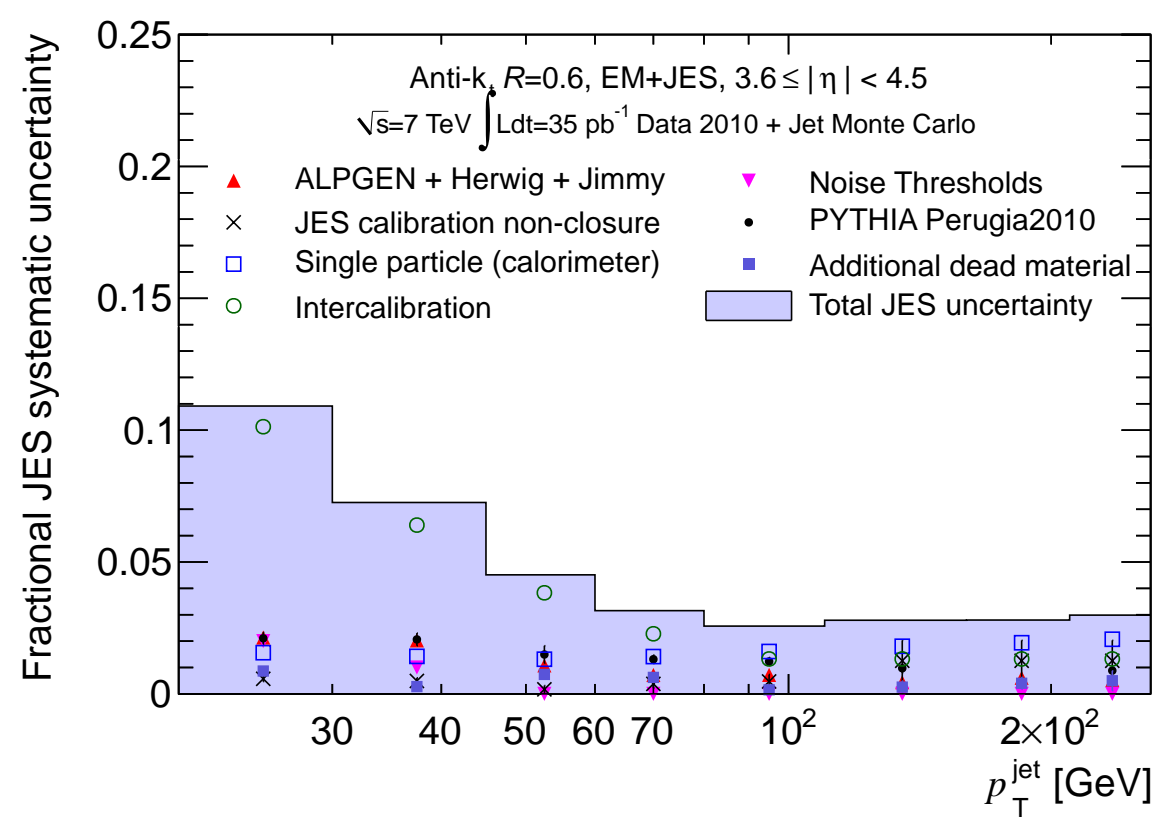

Figure 5.14.: Fractional jet energy scale systematic uncertainty as a function of $p_{\mathrm{T}}^{\text {jet }}$ for jets in the pseudorapidity region $3.6<|\eta|<4$.5. The JES uncertainty for the forward region is extrapolated from the barrel uncertainty, with the uncertainty contribution from the $\eta$ intercalibration between central and forward jets in data and Monte Carlo added in quadrature. The total uncertainty is shown as the solid light blue area. The individual sources are also shown, with uncertainties from the fitting procedure if applicable.

uncertainty is dominated by the modelling of the soft physics in the forward region that is accounted for in the intercalibration contribution.

The dominant contribution to the uncertainty for jets with the highest transverse momenta measurable in ATLAS is the calorimeter uncertainty, and more specifically the uncertainty due to particles in jets with $p>400 \mathrm{GeV}$. As stated in Section 5.2.2 and in [128], this uncertainty contribution is estimated conservatively.

Table 5.3 presents a summary of the maximum uncertainties in the different $\eta$ regions for anti- $k_{t}$ jets with distance parameter of $R=0.6$ and with $p_{\mathrm{T}}^{\text {jet }}$ of $20 \mathrm{GeV}, 200 \mathrm{GeV}$ and $1.5 \mathrm{TeV}$ as an example.

The same study has been repeated for anti- $k_{t}$ jets with distance parameter $R=0.4$, and the estimate of the JES uncertainty is comparable to anti- $k_{t}$ jets with $R=0.6$. The JES uncertainty for anti- $k_{t}$ jets with $R=0.4$ is between $\approx 4 \%(8 \%, 14 \%)$ at low jet $p_{\mathrm{T}}^{\text {jet }}$ and $\approx 2.5-3 \%(2.5-3.5 \%$, $5 \%$ ) for jets with $p_{\mathrm{T}}>60 \mathrm{GeV}$ in the central (endcap, forward) region, and it is summarised in Table 5.4. 


\subsubsection{Validation of the JES uncertainty with in-situ techniques}

A JES uncertainty that relies on Monte Carlo simulation for a certain number of terms benefits from validation of the jet energy scale with in-situ techniques that can exploit the full 2010 dataset. These techniques have been detailed in Section 4.2 and Appendix A. The comparison of data to Monte Carlo simulation for all in-situ techniques is shown in Figure 5.15, together with the JES uncertainty for the $0 \leq|\eta|<1.2$ region as estimated from the single hadrons response and systematic variations of the Monte Carlo simulations. For the track-jet results the $\eta$ range used for the comparison in the figure is restricted to $0 \leq|\eta|<0.3$. The results of the in-situ techniques provide independent support to the estimate of the JES uncertainty adopted by ATLAS.

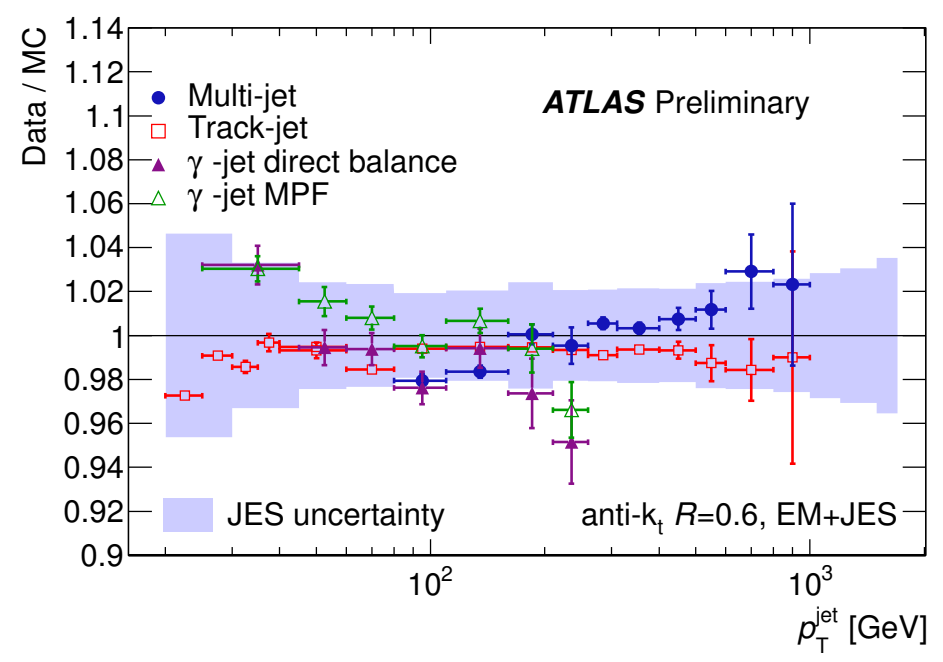

Figure 5.15.: Jet energy scale uncertainty as a function of $p_{\mathrm{T}}^{\text {jet }}$ in $0 \leq|\eta|<1.2$. This plot shows the data to Monte Carlo simulation ratios for several in-situ techniques that test the jet energy scale exploiting photon jet balance (direct balance or using the missing transverse momentum projection technique), the balance of a leading jet with a recoil system of two or more jets at lower transverse momentum (multi-jets) or using the momentum measurement of tracks in jets.

\subsection{Flavour and topology considerations}

The jet energy scale and its uncertainty have been derived using the nominal simulated sample of QCD jets. The calibration of the jet energy scale is constructed such that it restores the average jet response to unity for jets with the particular quark and gluon mixture and topologies predicted by the Monte Carlo simulation in di-jet QCD events. Therefore, differences in fragmentation between quark and gluon initiated jets and effect of close-by jets give rise to a 
particular topology and flavour dependence of the energy scale. Since the event topology and flavour composition (quark and gluon fractions) may be different in final states other than the QCD jets considered, the dependence of the jet energy response on jet flavour and topology has to be accounted for in physics analyses.

The effect of close-by jets on the jet energy response for a Monte Carlo sample with characteristics similar to the nominal sample of Section 1.4.2, but with an older ATLAS tune called MC09 [133], is shown in Figure 5.16 for anti- $k_{t}$ jets with distance parameter $R^{\text {jet algo }}=$ 0.6. The response of non-isolated jets $(\Delta R$ (jet, closest jet $)>1.5)$ is lower than the response of isolated jets, and strongly depends on the distance to the closest reconstructed jet. When $\Delta R$ (jet, closest jet) $<1.0$, the calibrated jet energy response is underestimated by $5 \%$ to $10 \%$. This same effect is observed in all $\eta$ regions. Isolated jets with $\Delta R$ (jet, closest jet) $<1.5$ have, by construction, a uniform response consistent with one: only isolated jets with $\Delta R<2.5 \cdot R^{\text {jet algo }}$ were used to derive the JES.

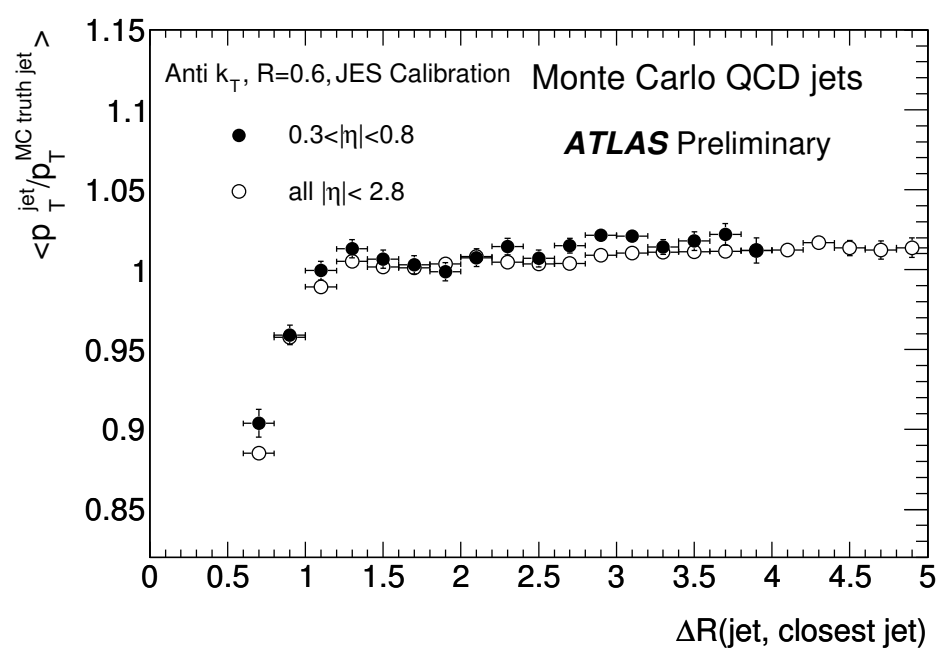

Figure 5.16.:Jet energy response after the JES calibration as a function of the distance to the closest jet in the event for jets in the central barrel (solid circles) region and all jets (open circles), from Monte Carlo simulation of the nominal sample.

The effect on the jet energy scale uncertainty due to close-by jets needs to be estimated separately, since the jet response depends on the angular distance to the closest jet. The response of jets as a function of the distance to the closest reconstructed jet needs to be studied and corrected for separately if the measurement relies on the absolute jet energy scale. The additional uncertainty can be estimated from the Monte Carlo to data comparison of the $p_{T}$ ratio between calorimeter jets and matched track jets in inclusive dijet events as a function of the isolation radius. The derivation of such uncertainty is described in Reference [134]: it is 
found that simulated events reproduce the response of close-by-jets to within 1-3\% as a function of the distance to the nearest jet.

Figure 5.17 shows the dependence of the jet energy response on the jet flavour in the nominal QCD jet sample. Gluon initiated jets have a lower response than quark jets because gluon jets fragment into more and softer particles than quark jets. Softer particles have a lower energy response and are more likely to fail to reach the calorimeters or be included in topological clusters.

The $p_{T}$ dependence of the jet response for quark and gluon jets reflects the flavour composition of the QCD dijet sample used to derived the JES. At low $p_{T}$, QCD di-jet production is dominated by gluon initiated jets. Hence, the response of gluon jets is consistent with unity. As the jet $p_{T}$ increases, there is a higher fraction of quark initiated jets in the QCD sample, resulting in relatively lower response for gluon jets. The maximum difference between the response of quark and gluon jets is about $6 \%$ at low $p_{\mathrm{T}}^{\text {jet }}$ and less than $3 \%$ for $p_{\mathrm{T}}^{\text {jet }}>100 \mathrm{GeV}$.

As in the case of the topology dependence of the jet energy scale, specific flavour dependent corrections should be derived separately by different physics analysis. The JES systematic uncertainty for each analysis depends on the difference in the fraction of quark and gluon jets and on the flavour dependence of the jet energy response between data and simulation. This uncertainty has to be evaluated for each individual physics analysis, as detailed in Reference [135].

\subsection{JES uncertainty correlations}

The treatment of correlated systematic uncertainties is of particular relevance for the comparison of experimental measurements with theory using statistical techniques. The JES uncertainty is composed of a number of uncorrelated sources, but no information has yet been given on the bin-to-bin correlations. This section focuses on the evaluation of the bin-to-bin correlations across $p_{T}$ bins.

In the approach taken for the inclusive jet cross section measurement described in this thesis, the full JES uncertainty covariance matrix is reproduced by treating each source as a nuisance parameter fully correlated across $p_{T}$. Each element of the covariance matrix $c_{i j}$ is given by:

$$
c_{i j}=s_{i} s_{j} \delta_{i j}+\sum_{\lambda}^{\text {sources }} \sigma_{i}^{\lambda} \sigma_{j}^{\lambda},
$$




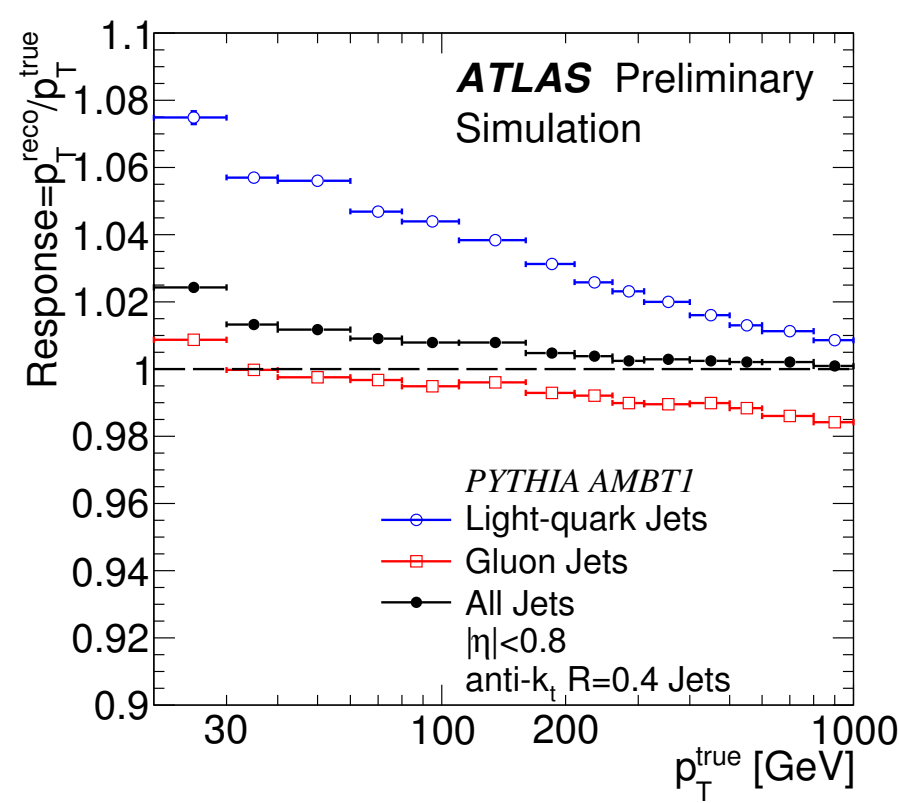

Figure 5.17.:Jet energy response after the JES calibration as a function of $p_{\mathrm{T}}^{\text {jet }}$ for light-quark (blue circles) and gluon (red squares) initiated jets shown together with the inclusive jet response (black circles), for the Monte Carlo simulation of the nominal sample. Figure from [135].

where $i, j$ are the indices of the $p_{T}$ bins considered, $s$ is the sum in quadrature of the uncertainties that are considered uncorrelated and $\sigma^{\lambda}$ are the individual sources of systematic uncertainties.

In order to adopt this treatment, the decomposition of the uncertainty sources needs to be revisited so that each uncertainty component can be treated as fully correlated in $p_{T}$. This assumption is made in the case of the uncertainties due to theoretical modeling, additional dead material ${ }^{6}$, noise thresholds, pile-up, non-closure of the calibration and relative calibration. On the other hand, the calorimeter component presents non trivial decorrelations between the $p_{T}$ bins, as derived from the analysis and shown in Figure 5.4. The decomposition of this uncertainty source into fully correlated components is described in the following section.

\subsubsection{Calorimeter uncertainty}

The technique adopted to reproduce the covariance matrix of the calorimeter uncertainty component using fully correlated subcomponents requires a revision of the seven uncertainty subcomponents listed in Section 5.2.2, as described below.

\footnotetext{
${ }^{6}$ Preliminary studies have been performed to estimate the bin-to-bin correlations for the dead material component using separate subsamples. These are not reported here, but they can be found in the presentation given in [136].
} 


\section{Treatment of bias components}

As described in Section 5.2.2, the full envelope of the bias and the deviation from unity is taken as the calorimeter uncertainty: the same technique is used when considering each separate source. The E/p and Combined Test Beam uncertainty sources have a significant bias term that dominates at high transverse momenta and needs to be treated separately from the term derived from the statistical and systematic error on the average JES (called error term in the following). Two separate subcomponents for the bias and error terms are constructed for each of these two sources using the total magnitude of the uncertainty $\sigma_{t o t}$ and the size of the error term $\sigma_{e r r}$ :

$$
\sigma_{\text {bias }}=\sqrt{\sigma_{\text {tot }}^{2}-\sigma_{\text {err }}^{2}}
$$

so that they can be summed in quadrature and treated as fully correlated across $p_{T}$.

\section{Further decomposition of calorimeter subcomponents}

While the uncertainties from the E/p acceptance, Neutral particles, cluster thresholds, E/p, High momentum particles sources can be treated as fully correlated, the Global energy scale and Combined Test Beam components have internal decorrelations. Their covariance matrix can be used to split them further in subcomponents using an iterative method that at each step subtracts a fully correlated part to the total uncertainty. By construction, the sum in quadrature of the subcomponent results in the total uncertainty for the original component. This is done as follows:

1. The first subcomponent for bin $i$ is derived using the elements of the covariance matrix $c_{l m}$, with $l, m$ bin indices. One bin (denoted with index $k$ ) is taken as reference ${ }^{7}$. The values of the new uncertainty component is derived using the covariance with respect to the reference bin as $\sigma_{i}^{(1)}=c_{i k} / \sqrt{c_{k k}}$;

\footnotetext{
${ }^{7}$ The reference bin is chosen where the uncertainty is largest to keep the correlations as faithful as possible in the neighboring bins.
} 
2. The modified covariance matrix with elements $c_{i j}^{(n)}$ where the $n$-1-th subcomponent has been subtracted is used to generate the other subcomponents:

$$
\begin{array}{r}
c_{i j}^{(n)}=c_{i j}^{(n-1)}-\sigma_{i}^{(n-1)} \sigma_{j}^{(n-1)} \\
\sigma_{i}^{(n)}=c_{i k^{\prime}}^{(n)} / \sqrt{c_{k^{\prime} k^{\prime}}^{(n)}}
\end{array}
$$

3. The two steps above are iterated until significant decorrelations are seen in the modified covariance matrix, and finally the last uncertainty component is built:

$$
\sigma_{i}^{(n)}=\sqrt{c_{i i}}-\sum_{n=1}^{N-1}\left(\sigma_{i}^{(n)}\right)^{2}
$$

This procedure leads to splitting the global energy scale component in two subcomponents. For convenience, these will be called in the following LAr E-scale and Tile E-scale, even though they are a mixture of uncertainties due to both the LAr and the Tile energy scales. The Combined Test Beam component is split into three subcomponents: CTB low $p_{T}$, CTB medium $p_{T}$ and CTB high $p_{T}$. Again, the names only indicate the $p_{T}$ range where the subcomponent is sizeable with respect to the others.

The correlation matrix for these two sources can be built using the new subcomponents and compared with that obtained from pseudoexperiments: the correlations are preserved within a few percent, and they are well reproduced at high $p_{T}$ where the two components are relevant in the total calorimeter uncertainty.

\section{Final calorimeter subcomponents}

As a result of this analysis, the calorimeter component is split into 12 subcomponents: 5 fully correlated 'original' components, 2 bias terms and 5 generated terms. The E/p acceptance and cluster thresholds components have a similar shape and they are merged in a single component. The same is done with the LAr E-scale, CTB low $p_{T}$ and neutral particles components and with the CTB high $p_{T}$ and high momentum particle components. A total of 7 uncertainty components are kept to be used as separate nuisance parameters for physics analyses: E/p, Cluster thresholds and E/p acceptance (called only Cluster thresholds in the following), LAr E-scale/neutrals and CTB low $p_{T}$ (called only LAr E-scale in the following), 
Tile E-scale and CTB mid $p_{T}$ (called only Tile E-scale in the following), CTB High $p_{T}$ and high momentum particles (called only CTB High $p_{T}$ in the following), E/p bias, CTB bias.

The correlation matrix obtained using these components is compared to those obtained in the full single particle analysis of Reference [128], and found in agreement up to $2 \%$ for $p_{T}>20$ $\mathrm{GeV}$, and $4 \%$ for the first bin. 
Table 5.3.:Summary of the maximum EM+JES jet energy scale systematic uncertainties for different $p_{\mathrm{T}}^{\text {jet }}$ and $\eta$ regions from Monte Carlo-based study for anti- $k_{t}$ jets with $R=0.6$.

\begin{tabular}{|c|c|c|c|}
\hline $\boldsymbol{\eta}$ region & \multicolumn{3}{|c|}{ Maximum fractional JES Uncertainty } \\
\hline & $\boldsymbol{p}_{\boldsymbol{T}}^{\text {jet }}=\mathbf{2 0} \mathbf{~ G e V}$ & $\boldsymbol{p}_{\boldsymbol{T}}^{\text {jet }}=\mathbf{2 0 0} \mathbf{~ G e V}$ & $\boldsymbol{p}_{\boldsymbol{T}}^{\text {jet }}=\mathbf{1 . 5} \mathbf{~ T e V}$ \\
\hline $0 \leq|\eta|<0.3$ & $4.6 \%$ & $2.3 \%$ & $3.1 \%$ \\
$0.3 \leq|\eta|<0.8$ & $4.5 \%$ & $2.2 \%$ & $3.3 \%$ \\
$0.8 \leq|\eta|<1.2$ & $4.4 \%$ & $2.3 \%$ & $3.3 \%$ \\
$1.2 \leq|\eta|<2.1$ & $5.4 \%$ & $2.4 \%$ & $3.4 \%$ \\
$2.1 \leq|\eta|<2.8$ & $6.5 \%$ & $2.5 \%$ & \\
$2.8 \leq|\eta|<3.2$ & $7.9 \%$ & $3.0 \%$ & \\
$3.2 \leq|\eta|<3.6$ & $8.1 \%$ & $3.0 \%$ & \\
$3.6 \leq|\eta|<4.5$ & $10.9 \%$ & $2.9 \%$ & \\
\hline
\end{tabular}

Table 5.4.:Summary of the maximum EM+JES jet energy scale systematic uncertainties for different $p_{\mathrm{T}}^{\mathrm{jet}}$ and $\eta$ regions from Monte Carlo based study for anti- $k_{t}$ jets with $R=0.4$.

\begin{tabular}{|c|c|c|c|}
\hline $\boldsymbol{\eta}$ region & \multicolumn{3}{|c|}{ Maximum fractional JES Uncertainty } \\
\hline & $\boldsymbol{p}_{\boldsymbol{T}}^{\text {jet }}=\mathbf{2 0} \mathbf{~ G e V}$ & $\boldsymbol{p}_{\boldsymbol{T}}^{\text {jet }}=\mathbf{2 0 0} \mathbf{G e V}$ & $\boldsymbol{p}_{\boldsymbol{T}}^{\text {jet }}=\mathbf{1 . 5} \mathbf{~ T e V}$ \\
\hline $0 \leq|\eta|<0.3$ & $4.1 \%$ & $2.3 \%$ & $3.1 \%$ \\
$0.3 \leq|\eta|<0.8$ & $4.3 \%$ & $2.4 \%$ & $3.3 \%$ \\
$0.8 \leq|\eta|<1.2$ & $4.4 \%$ & $2.5 \%$ & $3.4 \%$ \\
$1.2 \leq|\eta|<2.1$ & $5.3 \%$ & $2.6 \%$ & $3.5 \%$ \\
$2.1 \leq|\eta|<2.8$ & $7.4 \%$ & $2.7 \%$ & \\
$2.8 \leq|\eta|<3.2$ & $9.0 \%$ & $3.3 \%$ & \\
$3.2 \leq|\eta|<3.6$ & $9.3 \%$ & $3.5 \%$ & \\
$3.6 \leq|\eta|<4.5$ & $13.4 \%$ & $4.9 \%$ & \\
\hline
\end{tabular}




\section{Chapter 6.}

\section{Inclusive Jet Cross Section}

QCD jets are the most common hard objects observed at hadron colliders, with their cross section exceeding any other physics process by orders of magnitude.

The measurement of the inclusive jet cross section with the ATLAS detector has been one of the first measurements performed with the initial $7 \mathrm{TeV}$ data delivered by the Large Hadron Collider. Its first iteration used $17 \mathrm{nb}^{-1}$ of data collected between March 2010 and June 2010 including jets with transverse momentum from $60 \mathrm{GeV}$ to around $600 \mathrm{GeV}$ in a rapidity range of $y<2.8$, and it has been published in EPJC [53]. The comparison of the measurement data with the theoretical prediction served the purpose of both verifying Quantum Chromodynamics in an unexplored kinematic region and confirming the status of the performance of jets as estimated over the course of the experiment commissioning and with the data from the LHC startup.

The inclusive jet cross section measurement has been updated using the full 2010 dataset $\left(37.3 \pm 1.2 \mathrm{pb}^{-1}\right)$ and it is the focus of this chapter. This analysis takes advantage of the reduced jet energy scale uncertainty and the estimate of its correlations described in Chapter 5: its systematic uncertainties are considerably reduced compared to the first iteration. The kinematic range for the measurement has been extended to include jets from transverse momentum as low as $20 \mathrm{GeV}$ and up to $1.5 \mathrm{TeV}$, and measuring the jet cross section in the forward calorimeter region $(y<4.4)$. The comparison of the kinematic reach of the measurement for the full 2010 dataset compared to that of the previous measurement is shown in Figure 6.1.

This extension makes the measurement truly unique with respect to previous analyses [30,31] since it probes QCD at distances never reached before. The extension to the forward region provides insight in the small- $x$ QCD dynamics, while high $p_{T}$ jets allow constraints of the gluon density at high $x$. Low transverse momentum jets measured with two different jet distance 
parameters can be used to probe non perturbative effects such as hadronisation and underlying event.

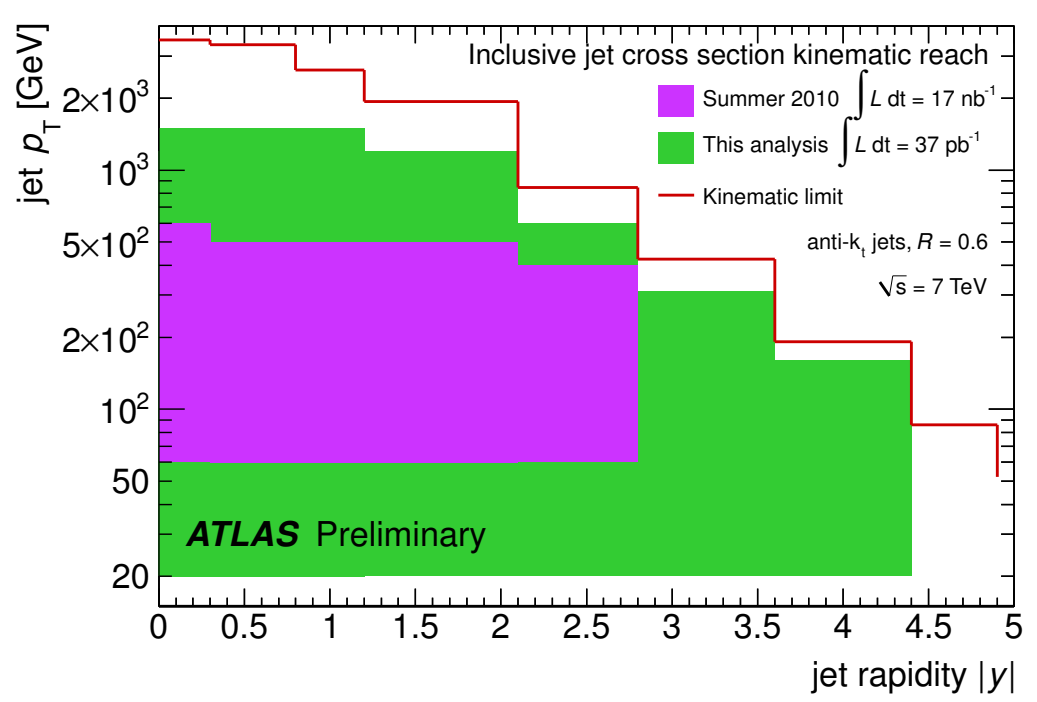

Figure 6.1.:Kinematic reach of the inclusive jet cross section measurement for the full 2010 dataset (green) described in this thesis, compared to that of the first iteration of the analysis reported in [53] (purple). Figure taken from Reference [137].

This chapter describes the details of the inclusive jet cross section analysis. The overview of the analysis and the datasets used are described in Section 6.1. The selection of interesting events and of the jets included in the measurement can be found in Section 6.2. The procedure needed to unfold the experimental effects from the measurement in order to compare to theory is outlined in Section 6.3, and the systematic uncertainties and their correlations are evaluated in Section 6.4. Section 6.5 briefly describes the theoretical prediction of the inclusive jet cross section. The comparison of data and theory and a brief discussion of the results are given in Section 6.6.

\subsection{Analysis overview and datasets used}

Jets are reconstructed with two different jet distance parameters $(R=0.4$ and $R=0.6)$ and calibrated with the EM+JES scheme described in Chapter 3. The measurement uses jets with a transverse momentum of $20<p_{\mathrm{T}}^{\text {jet }}<1500 \mathrm{GeV}$ and rapidities of $y<4$.4. The measurement is performed in bins of $p_{\mathrm{T}}^{\text {jet }}$ and $|y|$. The rapidity bins follow closely those used for the jet energy scale uncertainty, given that the these bins reflect the geometry of the ATLAS calorimeters. The differences with respect to Table 5.1 are that:

- pseudorapidity is replaced with rapidity; 
- the transition region is extended to include the first forward bin, leading to a transition region bin of $2.8<|y|<3.6$ to increase the statistics in a kinematic region where the spectrum falls steeply;

- the forwardmost bin is limited to $|y|<4.4$ instead of 4.5 .

The jet cross section $\sigma_{j e t}$ is defined as the number of jets $N_{\text {jets }}$ falling in a given $p_{T}, y$ bin after trigger and quality selection, fully corrected for experimental inefficiencies $\epsilon$ (as detailed in the following sections), and finally divided by the integrated luminosity $\mathcal{L}_{\text {int }}$ delivered by the Large Hadron Collider that takes into consideration any prescaling in the triggers used. The basic formula for the cross section measurement is shown in Equation 6.1.

$$
\sigma_{j e t}=\frac{N_{\text {jets }}}{\epsilon \cdot \mathcal{L}_{\text {int }}}
$$

All experimental effects (for example the smearing due to resolution) are corrected for using using a procedure (unfolding) that employs the Monte Carlo truth jets that are described in Section 3.1.3, but this time including muons and neutrinos from hadronic decays as specified in Reference [102].

The unfolded measurement is compared to Next to Leading Order (NLO) pQCD predictions corrected for non-perturbative effects and to NLO calculation that includes the simulation of the parton shower.

The measurement uses the full 2010 dataset with two exceptions:

- Since the forward jet triggers were not commissioned until August 10, 2010, only the latter data periods were used in the forward region $(|y|>2.8)$ and for $p_{\mathrm{T}}^{\text {jet }}>30 \mathrm{GeV}$;

- To minimize the effect of additional energy in jets due to in-time pile-up, only the first data periods (up to the beginning of June 2011) are used for jets with $20<p_{\mathrm{T}}^{\text {jet }}<60 \mathrm{GeV}$;

The total integrated luminosity used for this measurement is $(37.3 \pm 1.2) \mathrm{pb}^{1}$.

\subsection{Event and jet selection}

The Data Quality framework $[138,139]$ ensures that quality criteria for the subdetectors and the physics objects used in ATLAS analyses are met. Unrecoverable problems that affect the relevant detector components for a significant period of time and that can be observed at the 
luminosity block (defined in Section 2.8) level lead to the exclusion of the full range of events within the luminosity block from the analysis. Less frequent problems (such as sporadically noisy channels in the calorimeters) are instead dealt with at the analysis level, as detailed in Section 6.2.2. The quality selection performed at the event level for this measurement is detailed in Reference [140] and outlined below.

Events that are included in the analysis are required to belong to runs taken over periods of stable LHC beams. To ensure that the physics objects needed for the analysis are correctly measured, a further selection on the good functioning of the relevant detector components and reconstruction procedures is applied.

The subsystems that are required to measure the jets and the primary vertex used in the calibration procedure are the processors for the first level (L1) and second level (L2) triggers, the solenoid magnet, all components of the inner detector, and the LAr and Tile calorimeters. The reconstruction of physics objects needed in the analysis is also monitored: tracks (for the reconstruction of the primary vertex of the collision), jets and missing energy (to monitor the overall energy reconstruction) are required to be well reconstructed. A given luminosity block is excluded from the analysis if the distributions of significant quantities for the object in question are in disagreement with the expected reference distribution coming from a known good data taking period.

In addition to these requirements, cosmic muons and beam background (non-collision events) need to be rejected. To ensure that the event is a collision candidate, events are required to have at least one primary vertex (formed by at least five tracks) whose position is consistent with the beamspot.

\subsubsection{Triggers}

Three different trigger systems (described in more detail in Section 2.9) have been used to select the events used in the inclusive jet cross section analysis.

For the first data period (up to the 10th of April 2011), only the Minimum Bias Trigger Scintillators were used. This trigger is fully efficient for low $p_{T}$ jets in the whole calorimeter coverage, thanks to the particles radiated by the jets or by the proton remnants in the forward direction that cross either of the scintillator counters, as described in [141]. The MBTS trigger is also used to trigger low $p_{T}$ jets in the transition and forward calorimeter region. The central and forward jet trigger systems were also used: the Level 1 trigger was commissioned first, and subsequently (starting from August 2010) the Level 2 trigger was also used to reject events. 
The Level 1 jet trigger decision is based on whether the electromagnetic scale energy of any jet in the event is above a certain threshold. These thresholds are used because of the steep jet cross section: since the trigger system has a limited bandwidth as described in Section 2.9, the rate of events with low $p_{T}$ jets needs to be prescaled to ensure the less frequent high $p_{T}$ jets are recorded as well. 8 thresholds are available for the central jet trigger $(5,10,15,30,55,75$, 95 and $115 \mathrm{GeV}$ ) and 4 thresholds for the forward jet trigger (10, 30, 55 and $75 \mathrm{GeV})$. Level 2 triggers need to be seeded by a Level 1 trigger, which means that the event needs to have successfully passed the corresponding Level 1 trigger selection. Level 2 jet triggers also accept events based on EM-scale thresholds, which are $15 \mathrm{GeV}$ higher than their corresponding L1 threshold with the exception of the $\mathrm{L} 1$ central jet trigger with a $5 \mathrm{GeV}$ threshold that seeds a L2 trigger with a threshold of $10 \mathrm{GeV}$. A higher threshold at L2 with respect to L1 is used because the $\mathrm{L} 1$ trigger is slower at becoming fully efficient in the jet selection with respect to the L2 trigger, and this choice allows both trigger levels to become fully efficient at the same jet $p_{T}$ value.

The available bandwidth of about $200 \mathrm{~Hz}$ needs to be distributed among the various physics streams, so the jet triggers have been increasingly prescaled: only a fraction of the events that would be selected are saved, based on a probabilistic, unbiased selection. The prescale is accounted for when calculating the equivalent luminosity from a given trigger, by weighting the event by the inverse of the prescale factor.

The trigger strategy for the inclusive jet measurement is to accept any jets in events where at least one jet passed a trigger. The definition of trigger efficiency follows this choice: it is calculated as the ratio between all jets in an event where at least one of the jets fired the trigger and all jets in all events considered, where the latter jets are counted using a trigger chain with a lower threshold. This definition requires no matching between jets triggered online and jets recorded offline, since there might be no connection between the jet that caused the event to be selected and the jet that enters the measurement. The efficiency of a L2 trigger needs to be combined with the efficiency of the seeding L1 trigger. A trigger is considered fully efficient at the calibrated $p_{T}$ where its efficiency reaches the $99 \%$ plateau. A sample of trigger efficiency curves are shown in Figure 6.2 for the central region and for the forward region ${ }^{1}$.

An association between trigger passed and calibrated jet momentum is made in order to minimize statistical uncertainties due to trigger efficiency: each $p_{\mathrm{T}}^{\text {jet }}$ bin only contains jets coming

\footnotetext{
${ }^{1}$ Due to a known problem that caused a trigger tower to be unusable in the FCal, the efficiency of some chains does not reach $100 \%$ efficiency. Since the effect of the dead trigger tower on the trigger efficiency is below $1 \%$ because jets in that region can still be recorded thanks to other jets triggering the event, no offline cuts are applied, and this effect is considered as a systematic uncertainty.
} 


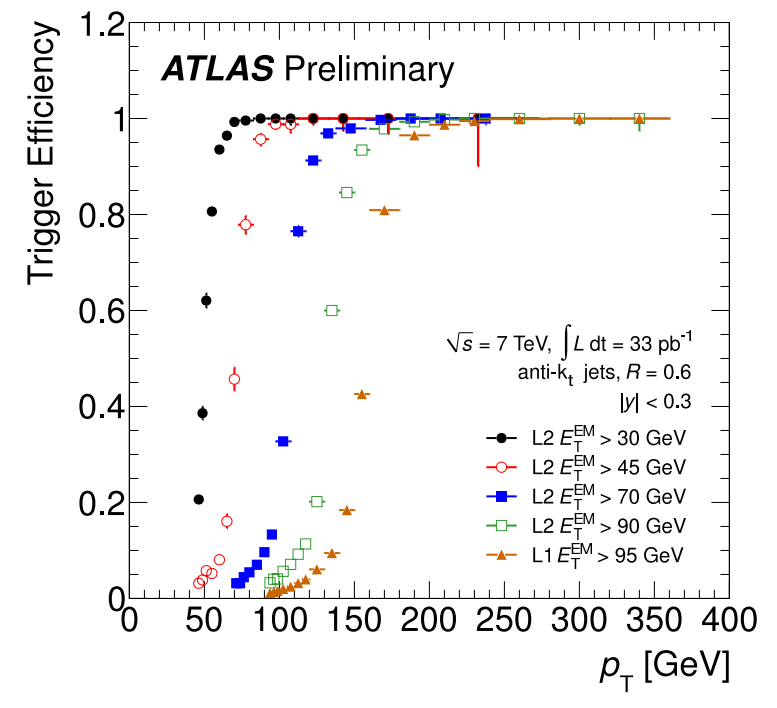

(a)

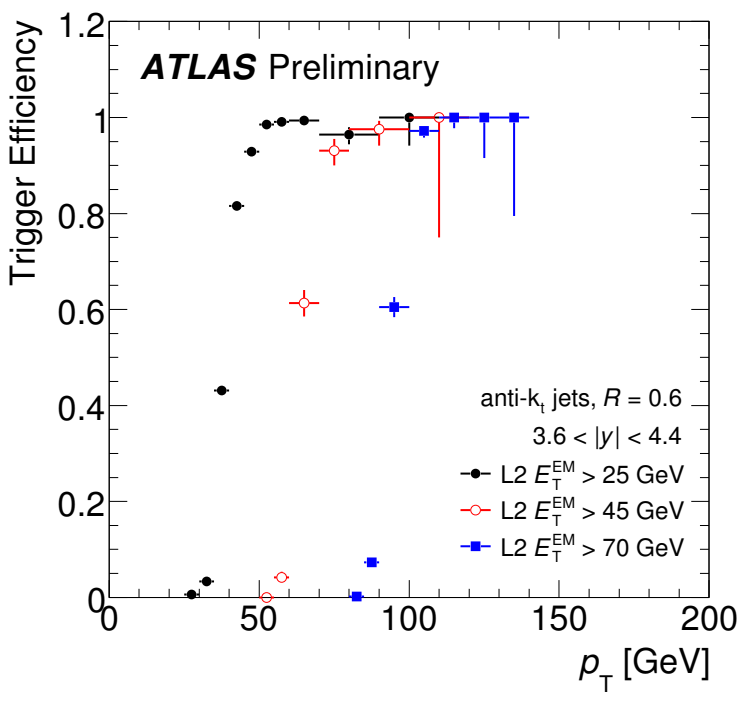

(b)

Figure 6.2.:Trigger efficiencies for a the L1-L2 chains used in the inclusive jet measurement, for anti- $k_{t} R=0.6$ jets in the $y<0.3$ (a) and $3.6<y<4.4$ (b) regions. Figure from [53].

from events selected by a trigger that is fully efficient at that value of calibrated $p_{T}$. When multiple triggers satisfy this criterion, the trigger with the lowest $p_{T}$ threshold is selected, in order to maximise the available statistics. For the endcap/forward transition region, the OR of a central and forward trigger is taken to obtain a fully efficient trigger combination. A full list of the bin-trigger association is shown in Reference [142].

To account for the equivalent luminosity and avoid double counting in the case when a jet is in an event that has been triggered by both the central and the forward jet trigger, jets are divided in three categories: triggered by a central jet trigger only, triggered by a forward jet trigger only, and triggered by a central and a forward jet trigger. The final luminosity is calculated taking into account the integrated luminosity recorded for the trigger and the prescale of each trigger for the three categories, according to the prescription in Reference [143].

\section{Impact of events in the trigger debug stream}

Any events for which the trigger is unable to make a decision because either the processing time was too long (trigger time-out) or they caused a failure in the online software are redirected to a special stream called debug stream. In the case of the inclusive jet measurement these events are particularly relevant since they could be high- $p_{T}$ jets in poorly instrumented calorimeter regions (e.g. barrel/endcap crack): even if their number is negligible (less than 1 per mille) they could cause a bias in the final jet spectrum. These jets are not fully contained within 
the calorimeters and 'punch-through' to the muon system, causing the muon reconstruction (present in the L2 trigger) to reach the maximum allowed processing time. A separate study of punch-through events has been performed and it is detailed in Reference [144], showing that these occurrences are present and reasonably well modeled in Monte Carlo. Nevertheless, in order not to bias the measured jet spectrum (debug stream events usually contain jets that would populate the high $p_{T}$ tails of the spectrum), these jets have been included in the standard analysis and passed through the standard jet identification cuts mentioned below. A subset of these events were further checked using the ATLANTIS event display [145] and they were all found to contain real jets. 530 jets over 2.1 million jets

\subsubsection{Jet identification}

After jets have passed the trigger, fake jets are rejected from the sample used in the measurement using a series of jet-by-jet cleaning cuts. Fake jets include energy deposits coming from groups of noisy cells in the calorimeters and cosmic muons traversing the calorimeters in the same time window allowed for a collision event.

The basic cleaning cuts performed in analyses using jets are detailed in [140]. Specific studies have been performed for the inclusive jet measurement, also given that the reprocessing of the data used for the measurement allowed use of discriminating variables that were not available at the time of the first studies. Only the discriminating variables used in jet cleaning are mentioned in this section, while the specific cuts can be found in the full analysis documentation [142]. Cleaning cuts are targeting three main category of fake jets, and the discriminating variables to remove each category are listed in the following.

\section{Jets from noise bursts occurring in the HEC :}

- the fraction of energy deposited in the HEC (HECf),

- the HEC quality variable, defined as the fraction of cells in the HEC that present a pulse shape with large discrepancies with respect to a 'healthy' reference shape,

- the sum of all negative cell energies (in absolute value): cells with negative total energy are more likely to be coming from noise fluctuations, since the reconstruction of the pulse shape will not have recognized its shape correctly.

\section{Jets from coherent noise in the LAr calorimeter :}

- the fraction of energy deposited in the LAr (EMf), 
- the LAr quality variable, defined in the same fashion as the HEC quality variable.

\section{Jets from non-collision background, e.g. beam background or cosmic rays :}

- the fraction of energy deposited in the LAr (EMf),

- the sum of the $p_{T}$ associated to charged tracks divided by the total jet $p_{T}(\operatorname{chf})$,

- the difference in the timing of the jet (obtained as the average time of all cells weighted by the cell energy) with respect to the global event timing,

- the maximum fraction of energy deposited in any of the layers of the calorimeters.

Cleaning cuts were optimised using jets with a distance parameter of $R=0.6$, and the choices were tested with $R=0.4$ with no significant differences in the results. The optimisation of the cleaning cuts was performed on samples of real and fake jets, and by looking at the missing energy distributions before and after applying the cleaning cuts. Fake jets are not likely to have a counterpart that balances them in tranverse momentum, and therefore produce tails in the missing energy distributions. An example of the effect of the cleaning cuts on the missing transverse energy distribution in the case of the cuts mentioned in Reference [140] is shown in Figure 6.3

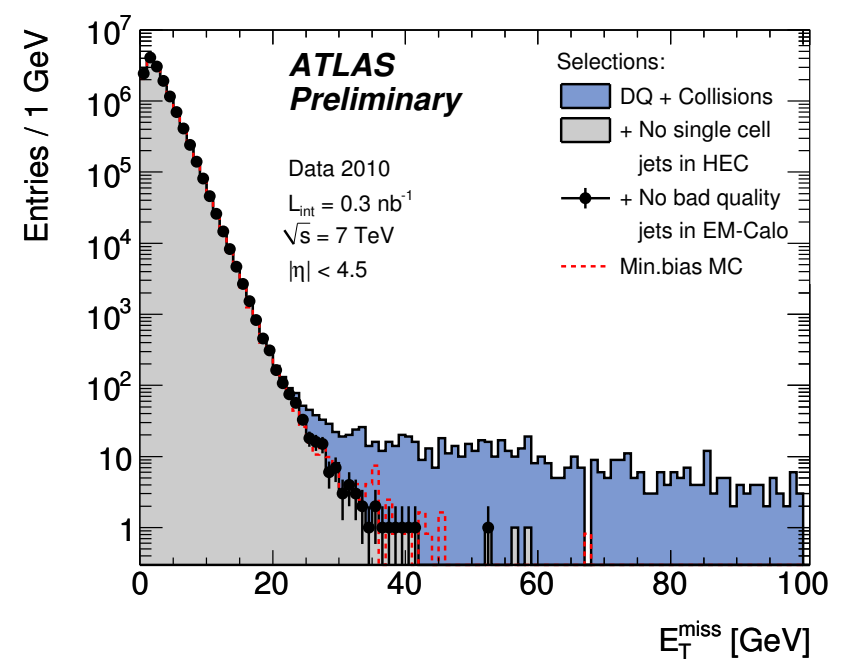

Figure 6.3.:Effect of the cleaning cuts on the $E_{T}^{\text {miss }}$ distribution (built from topoclustersat the EM scale) after successive jet cleaning selections. The normalized Monte Carlo distribution is overlaid to the data in the dashed red line. Figure taken from Reference [140].

The efficiency of the set of cleaning cuts is estimated using a tag and probe approach. Tag jets (considered to a good approximation to correspond to a sample of real jets) are selected with a series of cuts that are tighter with respect to the standard cleaning selection. The analysis looks 
for well balanced probe jets corresponding to tag jets before and after applying the cleaning selection, and measures the probability of the cleaning cuts to reject a real jet. The systematic uncertainty is derived applying a looser or harder tag selection.

Inefficiencies of $>1 \%$ (that can happen in the lowest $p_{T}$ bins since cleaning cuts are harder for low $p_{T}$ jets) are corrected for at the analysis level after unfolding ${ }^{2}$. An additional cross check of the cleaning efficiency was given by checking the fraction of jets rejected in Monte Carlo, where the problems that cause fake jets as are not present.

\subsubsection{Stability cross checks and effect of pile-up}

The conditions of the LHC varied considerably over the course of the 2010 data taking: both the instantaneous luminosity and the number of bunches per fill were increased. The jet yield after the cleaning and trigger selection was checked over all the runs used for the measurement. This study showed that the jet yield is generally stable within uncertainties across the data taking period, with the exception of a few isolated runs. It was also noticed that in the lowest $p_{\mathrm{T}}^{\text {jet }}$ bin $(20-30 \mathrm{GeV})$ the jet yield increased starting from the end of June 2011. Starting from this period, the number of protons for each bunch was increased to the nominal LHC value of $0.9 \cdot 10^{11}$ and the luminous region $\beta *$ decreased to $3.5 \mathrm{~m}$ : this lead to an average of 1.3 interactions per event compared to a value of $<0.15$ before these changes. In order to avoid an increased impact of pile-up in the measurement, it was decided to only use the first collision data (March/April 2010 to the $24^{\text {th }}$ of June 2010) for the first three bin of the measurement, up to $60 \mathrm{GeV}$. This decision is also supported by the large amount of statistics for low $p_{T}$ jets collected in this data period.

\subsection{Data corrections for detector effects (unfolding)}

A number of detector-related factors can distort the distribution of observables that are measured experimentally with respect to an ideal detector and infinite event statistics within the phase space of the measurement. Among these factors are the resolution of the detector, detector inefficiencies and the limited acceptance of the subdetectors used for the measurement. A meaningful comparison to theory, e.g. pQCD-corrected NLO calculations where no detector effects are present, is possible after a correction accounting for these effects has been applied. The procedure where these corrections are derived and applied is called unfolding. Unfolded

\footnotetext{
${ }^{2}$ As explained in Section 6.3.1, the unfolding procedure is based on the Monte Carlo simulation, where the number of fake jets is negligible.
} 
distributions are detector-independent, and can be used for theoretical comparisons, comparisons to results of other experiments and PDF fits.

A review of unfolding methods can be found in [146] and [147] in the specific case of the ATLAS experiment as of Winter 2010. In the following a summary of the unfolding methods employed for the inclusive jet measurement is given. The issues that could arise from background subtraction are not mentioned since the measurement has effectively a negligible background that is removed with the jet cleaning procedures detailed in Section 6.2.2.

The problem of unfolding an experimental measurement can be seen as trying to retrieve the probability distribution function of a given observable $y$, given a series of measurements $y_{1} \ldots y_{N}$ in $\mathrm{N}$ bins. The goal of the unfolding procedure is to construct estimators for the number of entries in each bin, free from any detector effects (called $\mu_{i}$ in the following, $\hat{\mu}_{i}$ if referring to the estimator). The number of events smeared by detector effects is indicated with $\nu_{i}$, and the connection between the smeared and unsmeared number of events is a response matrix that reflects the bin migrations:

$$
\nu_{i}=\sum_{j=1}^{M} R_{i j} \mu_{j} .
$$

The response matrix can then be inverted to revert to the number of events before any detector smearing. In practice, one only has the number of events measured in each bin $n_{i}$, that can be taken as estimators for $\nu$. Therefore, an unbiased estimator for $\nu_{i}$ can be constructed using $n_{i}{ }^{3}$. The estimator for the number of entries in a given bin $\hat{\mu}_{i}$ can be obtained as:

$$
\hat{\mu}=R^{-1} \mathbf{n}
$$

Since only limited statistics is available in experimental measurements, this direct matrix inversion procedure is unfeasible. The reason is that $R$ does not differentiate between real structures and statistical fluctuations, and it can lead to large fluctuations and large negative correlations in the values of $\hat{\mu}_{j}$ that are not physical.

A simple method to prevent these large variances is the so-called bin by bin unfolding, where the ratio of the number truth Monte Carlo objects $N_{\text {truth }}$ to the reconstructed Monte Carlo objects $N_{\text {reco }}$ is used to obtain $\hat{\mu}_{i}$ in each bin:

\footnotetext{
${ }^{3}$ This if the $n_{i}$ are independent and follow Poisson statistics with mean $\nu_{i}$.
} 


$$
\hat{\mu}=\frac{N_{\text {truth }}^{i}}{N_{\text {reco }}^{i}} n_{i}
$$

The inclusive jet cross section measurement with $17 \mathrm{nb}^{-1}$ and the first iteration of the analysis on the full 2010 dataset employ this unfolding method, using as unfolding correction coefficients the ratio between the number of truth (particle-level) jets derived from the Pythia nominal Monte Carlo sample described in Section 1.4.2 and the number of reconstructed jets.

The bin by bin unfolding method is the most widely used in ATLAS for its simplicity, but it biases the final measurement towards the Monte Carlo prediction. If the truth level spectrum is different from the 'real' truth spectrum (in nature), then the measurement will not necessarily reflect the true nature of QCD but will instead be biased towards the modelling of QCD that is embedded in the Monte Carlo generation.

Another unfolding technique that reduces the large variances induced by the use of estimators based on the inversion of the response matrix is the regularized unfolding. In this technique, the log-likelihood that represents the distance between the measured and true values of the observable (in terms of $\mu$ ) is constrained to be within a certain value from its maximum:

$$
\ln L(\mu) \geq \ln L\left(\mu_{\max }\right)-\Delta \ln L(\mu)
$$

which is equivalent to redefining the log-likelihood using a regularisation function $f$ multiplied by a parameter $\lambda$ :

$$
\ln L^{\prime}(\mu)=\ln L(\mu)+\lambda f(\mu)
$$

The regularisation function $f$ and the value of the parameter $\lambda$ need to be determined, and the choices used in the inclusive jet cross section measurement presented in this thesis follows those of the Iterative Dynamically Stabilized (IDS) unfolding method [148,149], described in the next section. The use of an unfolding method based on the inversion of the Monte Carlo transfer matrix is justified by the good modelling of the trigger efficiencies in the simulation and the good description of the energy and momentum flow around the jet core (jet shapes) for the full coverage of the calorimeters used in the measurement, as shown in References [53,132]. 


\subsubsection{Iterative Dynamically Stabilized (IDS) unfolding}

The IDS method $[148,149]$ is an unfolding technique that takes advantage of a series of iterations to improve the description of the Monte Carlo simulation according to data. In each step the method uses the information on the size of the experimental errors in the data to stabilize the inversion of the transfer matrix. This technique allows the Monte Carlo normalisation to be improved so that the final result is not biased, and keeps information on new structures that might not be simulated in the Monte Carlo without at the same time being affected by fluctuations. Its result is consistent with that derived using the bin-by-bin unfolding mentioned above, and has been cross checked using the SVD unfolding technique [142].

\section{Regularisation function and parameter}

The regularisation function used in the IDS unfolding technique uses the significance of the absolute deviation between data and the Monte Carlo to prevent spurious fluctuations (e.g. due to statistics) in the unfolded spectrum when iterating. This quantity is defined as $\Delta x / \sigma$, where $\Delta x$ is the deviation between data and Monte Carlo and $\sigma$ is the statistical error on the data point. The regularisation function is smooth and monotonic, and its value goes from zero to unity when the data/Monte Carlo difference is larger than the statistical error. The parameter $\lambda$ is included in the regularisation function, and chosen depending on the unfolding step: whenever the Monte Carlo shape needs to be taken as a reference, e.g. when computing the data/Monte Carlo normalisation factor, $\lambda$ is set to zero, while it takes larger values when the Monte Carlo needs to be improved taking into account the shape of the data.

\section{Unfolding procedure for the inclusive jet cross section}

The main ingredient for the unfolding procedure is the transfer matrix $A_{i j}$, which is derived from Monte Carlo and contains the number of jets that have been reconstructed in bin $i$ with a matched truth jet that was generated in bin $j$. A reconstructed and true jet are considered matched if their centers lie within $\Delta R<0.3$ of each other, and the matching is unique. The transfer matrix does not include unmatched jets, so an equivalent fraction of jets in data needs to be removed from the unfolding procedure: a multiplicative inefficiency equal to the fraction of unmatched jets is applied to data before the start of the unfolding procedure, and the equivalent number of jets is restored after the unfolding.

To improve the initial data/Monte Carlo agreement, the Monte Carlo spectrum is reweighted according to the distribution of the spectrum generated using a NLO PDF instead of the current 
LO*. This step improves the stability of the method, given that the regularisation function in the IDS unfolding accounts for the significance of the data/MC difference.

The matrix of unfolding probabilities is calculated from the transfer matrix in Monte Carlo as:

$$
\tilde{P}_{i j}=\frac{A_{i j}}{\sum_{k=1}^{N B i n s} A_{i k}}
$$

The data/Monte Carlo normalisation coefficient is also calculated counting the number of events in data. The use of the regularisation function for this step avoids inclusion of any new structures that are not simulated in the Monte Carlo (which are anyway not present in the inclusive jet cross section data spectrum) to calculate the normalisation.

In the case of negligible background as in the inclusive jet cross section measurement, the number of unfolded events for each bin is calculated from the sum of three contributions:

1. the normalized number of jets in the bin from the true spectrum,

2. the fractions of events that migrate from other bins according to their unfolding probabilities,

3. the fraction of events that remain in that same bin (these events are not unfolded).

The size of the last two contributions is controlled by the regularisation function and by the $\lambda$ parameter: the choice of function and the value of the parameter are determined using pseudo experiment (toy) studies. The optimisation is performed using a "closure test" of the unfolding procedure: the parameter $\lambda$ is chosen to be the one that minimizes the bias in a test of the unfolding where the true Monte Carlo spectrum is reweighted to data and unfolded, and finally compared to the original true Monte Carlo spectrum.

The unfolding procedure for the inclusive jet measurement is iterated once. The agreement between the truth distribution in the transfer matrix and the intermediate unfolded spectrum is improved using a fraction of events dictated by the regularisation function and parameter, and the final number of unfolded events is derived using the new transfer matrix.

\section{Treatment of statistical uncertainties}

The statistical uncertainties are propagated through the unfolding using a series of pseudo experiments in which the content of the bins of the transfer matrix from the Monte Carlo simulation are fluctuated according to a Poisson distribution. Correlations due to the production of jets within the same event are also considered: for example, in a dijet event the transverse 
momenta of the two jets entering the measurement are fully correlated, and using only the Poissonian error would lead to an underestimate of the statistical uncertainty. Each element of the covariance matrix $\operatorname{cov}_{i, j}$ for bins $i$ and $j$ of the jet spectrum is calculated from data taking into account correlations between jets in the same event:

$$
\operatorname{cov}(i, j)=\sum_{\text {events }}\left(\text { Njets }_{i} \cdot \text { Njets }_{j}\right)
$$

The covariance matrix is calculated for both data and Monte Carlo, and it is used to fluctuate the number of jets in 100 different pseudo experiments. For each fluctuated data spectrum and transfer matrix, the unfolding procedure is repeated, and the final statistical errors are derived from the diagonal elements of the covariance matrix derived from all pseudo experiments.

\subsection{Systematic uncertainties}

This section outlines the evaluation of the systematic uncertainties for the inclusive jet cross section measurement, source by source. Every single source of uncertainty is considered separately in order to be input either as a nuisance parameter for PDF fits or as a completely uncorrelated source, as described in Section 6.4.8.

\subsubsection{Jet energy scale uncertainty}

The evaluation of the jet energy scale uncertainty and its separate components has been detailed in Chapter 5. Each of the thirteen uncertainty sources mentioned in Section 5.7 (excluding the pile-up which is estimated separately) can be applied as a separate shift to the energy scale of each jet in the Monte Carlo spectrum that is used in the data unfolding. Since the uncertainty for each of the sources is considered fully correlated in $p_{\mathrm{T}}^{\text {jet }}$, all jets are shifted upwards and downwards in two separate steps. Given that the number of uncertainty components is large, a jet spectrum that is finely binned in jet transverse momentum is used for each rapidity region instead of shifting each jet in order to reduce the processing time. For each $i$-th jet energy scale uncertainty component, the fractional JES uncertainty $\delta_{J E S, i}$ for the $p_{T}$ bin centre $\left(p_{T}^{\text {bin centre }}\right)$ is used to move all jets in a given bin to the bin corresponding to:

$$
p_{T}^{\text {jet,up }}=p_{T}^{\text {bin centre }}+p_{T}^{\text {bin centre }} \cdot \delta_{J E S, i}
$$

in the case of the positive fluctuation, and to: 


$$
p_{T}^{\text {jet,down }}=p_{T}^{\text {bin centre }}-p_{T}^{\text {bin centre }} \cdot \delta_{J E S, i}
$$

in the case of the negative fluctuation. The procedure is repeated for all bin centers and the shifted spectrum is rebinned to the binning used in the measurement. The unfolding procedure described in Section 6.3.1 is used to propagate the uncertainty to the final measurement: the upwards/downwards shifted Monte Carlo spectrum are unfolded using the nominal transfer matrix, and compared to the nominal Monte Carlo spectrum after unfolding. The relative difference between the two spectra is taken as uncertainty. The relative upwards and downwards uncertainty at this stage are called $\delta_{J E S, i}^{x s e c, u p}$ and $\delta_{J E S, i}^{x s e c, d o w n}$. Since statistical fluctuations in the unfolding procedure might lead the spectrum to present a downwards fluctuation when shifted upwards (or vice versa), the final uncertainty is asymmetric and calculated in its upwards $\Delta_{J E S, i}^{u p}$ and downwards components $\Delta_{J E S, i}^{\text {down }}$ as:

$$
\begin{aligned}
\Delta_{J E S, i}^{u p} & =\max \left(\left(\max \left(\delta_{J E S, i}^{x s e c, u p}, \delta_{J E S, i}^{x s e c, \text { down }}\right), 0\right)\right. \\
\Delta_{J E S, i}^{\text {down }} & =\min \left(\left(\min \left(\delta_{J E S, i}^{x s e c, u p}, \delta_{J E S, i}^{x s e c, \text { down }}\right), 0\right)\right.
\end{aligned}
$$

The uncertainty on the measurement due to pile-up is estimated using the average vertex distribution in data for each $p_{\mathrm{T}}^{\text {jet }}$ bin in the measurement, where vertices are counted using the standard track selection described in Section 3.2.1. First the fractional uncertainty due to pile-up shown in Table 5.2 is averaged for a given $p_{T}, y$ bin over all values of $N_{\mathrm{PV}}$. The uncertainty applied to the measurement is calculated multiplying this average pile-up uncertainty by the fraction of events with a given number of $N_{\mathrm{PV}}$.

As shown previously in Figure 5.11, this uncertainty is most relevant at low $p_{\mathrm{T}}^{\text {jet }}$. Given that the jets in the transverse momentum bins with $p_{T}<60 \mathrm{GeV}$ have been chosen to exclusively come from periods in which the machine was running at a reduced luminosity, the impact of pile-up on the measurement is reduced, and it amounts to generally less than $1 \%$.

\subsubsection{Jet energy resolution uncertainty}

The data/Monte Carlo studies detailed in Section 4.1 showed that the jet resolution is well described in Monte Carlo with respect to data, to within an uncertainty of the order of $5-10 \%$. Fluctuations of the jet energy due to resolution effects are corrected for in the unfolding procedure, but a worse resolution with respect to what is modeled in the Monte Carlo simulation used for the unfolding can still lead to biases in the measurement, especially at low jet $p_{T}$. Low 
$p_{T}$ jets whose energy fluctuates above the nominal value will fall to higher $p_{T}$ bins, and this flux will not be compensated by jets in higher $p_{T}$ bins because of the steeply falling jet cross section.

In order to evaluate the systematic uncertainty due to a potentially worse resolution with respect to the Monte Carlo simulation, the nominal energy resolution is worsened to within its uncertainty. The $p_{\mathrm{T}}^{\mathrm{jet}}$ of each Monte Carlo jet is smeared by the factor $\sigma_{\text {smear }}$, calculated as:

$$
\sigma_{\text {smear }}^{2}-\sigma_{\text {nominal }}^{2}=(1+\Delta \sigma) \cdot \sigma_{\text {nominal }}
$$

where $\sigma_{\text {nominal }}$ is the nominal fractional resolution as derived from Monte Carlo studies in Section 4.3.3, and $\Delta \sigma$ the fractional uncertainty on the nominal resolution.

The smeared spectrum is used to derive a smeared transfer matrix with which the data is unfolded. The relative difference of the data spectrum unfolded with the smeared transfer matrix and the data spectrum unfolded with the nominal transfer matrix is taken as a symmetric systematic uncertainty. The maximum uncertainty on the inclusive jet measurement due to resolution effects amounts to up to $10 \%$ in the forwardmost, low $p_{T}$ bins.

\subsubsection{Jet identification and trigger}

The jet identification efficiency after jet cleaning is obtained with the tag and probe method described in Section 6.2.2, and the systematic uncertainty on the method is estimated by varying the tag selection to be tighter or looser with respect to what is used for the efficiency estimate. The systematic uncertainty due to jet identification is generally less than $1 \%$. The systematic uncertainty on the trigger selection is estimated from the difference in the trigger efficiencies measured in data and Monte Carlo, which is well reproduced over the full kinematic range of the measurement to $1 \%$ thanks to the strategy of employing jets from fully efficient triggers for the measurement. A flat systematic uncertainty of $1 \%$ due to trigger is assigned to the final inclusive jet cross section.

\subsubsection{Jet angular resolution uncertainty}

The uncertainty on the angular resolution is obtained by smearing the angle with respect to the beam axis $\theta$ for each jet by $10 \%$ of the width of the distribution of the difference of the truth and reconstructed $\theta$ in Monte Carlo. The final uncertainty on the measurement is estimated comparing the nominal inclusive jet spectrum and the spectrum built from smeared jets. The uncertainty on the final measurement due to the angular resolution is always less than $1 \%$. 


\subsubsection{Reconstruction efficiency}

The unfolding procedure will restore inefficiencies for jet reconstruction (e.g. due to the noisesuppression thresholds of topological clusters) based on the Monte Carlo simulation. A systematic uncertainty needs to be assigned only in the case of data/Monte Carlo disagreement in the reconstruction efficiency. This has been evaluated using track jets as detailed in Reference [144]: the efficiency of reconstructing calorimeter jets using track jets as reference objects has been measured in data and Monte Carlo, and the data/Monte Carlo have been found in agreement to $2 \%$ for jets in the lowest $p_{\mathrm{T}}^{\text {jet }}$ bin and to less than $1 \%$ elsewhere. A systematic uncertainty of $2 \%$ for jets from 20 to $30 \mathrm{GeV}$ and $1 \%$ for jets with $p_{\mathrm{T}}^{\text {jet }}>30 \mathrm{GeV}$ has been assigned. The uncertainty estimated with this method has been found to be a conservative envelope for the inefficiencies observed when using truth jets as reference objects over the whole rapidity range, as shown in Section 4.3.4, and it is therefore extended to the whole rapidity coverage of the measurement.

\subsubsection{Unfolding}

The contributions to the systematic uncertainties related to the unfolding procedure come from the differences in shape between data and Monte Carlo and from varying the criteria for matching reconstructed and truth jets to derive the transfer matrix.

The systematic uncertainty due to shape differences is evaluated by performing a reweighting of the true and reconstructed Monte Carlo spectra to be closer to the data. The modified reconstructed Monte Carlo is then unfolded using the same procedure that is applied to data, using the nominal unfolding matrix. The relative difference of this unfolding result with respect to the reweighted true Monte Carlo is taken as systematic uncertainty. This check also effectively provides a closure test for the unfolding procedure, and the limited size of the systematic uncertainty $(<2.5 \%$ for the whole range) shows that the method used is stable.

The transfer matrix is also recalculated using different values for the reconstructed/true jet matching $(\Delta R=0.4$ and 0.2 instead of 0.3$)$. The data is unfolded using the new transfer matrices and the relative difference with respect to the nominal unfolded data spectrum taken as a systematic uncertainty. This uncertainty is more relevant at low $p_{T}$ due to the lower matching efficiency, but since a fraction of the jets in data corresponding to unmatched jets is also corrected using the multiplicative reweighting mentioned in Section 6.3.1, the final impact on the measurement is always less than $1.5 \%$. 


\subsubsection{Luminosity measurement}

The uncertainty on the LHC luminosity measurement has been estimated using scans of the LHC beam position from October 2010 [59], and it amounts to $3.4 \%$.

\subsubsection{Overall systematic uncertainties and correlations}

The overall systematic uncertainty for three sample rapidity bins is shown as the shaded blue area in Figure 6.4 for jets with distance parameter $R=0.6$ (similar results are found for $R=0.4$ ). The blue line in the figures corresponds to the total JES systematic uncertainty, and it is the dominating component in the overall uncertainty due to the steeply falling jet spectrum. The uncertainty due to the jet energy resolution (red line) is relevant especially at low $p_{T}$ where the knowledge of the simulated resolution is poorer (as explained in Section 4.1), while the luminosity uncertainty dominates among the other uncertainty sources (orange line).

\section{Bin-to-bin correlations}

The largest uncertainty in the measurement is due to the JES uncertainty. As previously shown in Figures 5.12- 5.14, the dominant JES uncertainty components are the JES calorimeter uncertainty, the JES intercalibration uncertainty in the forward region and the jet energy resolution uncertainties: knowledge on the bin-to-bin correlation of these sources is particularly important to allow the use of this data in fits that compare data to theory and/or use this comparison to extract theory parameters. The $p_{T}$ and $\eta$ bin-to-bin correlations of the systematic uncertainty sources are estimated as in the following, and their treatment is summarised in Table 6.1.

JES: calorimeter uncertainties These sources are treated as fully correlated in $p_{T}$ and in $y$ up to $y<0.8$. In the central region, the breakdown of the calorimeter uncertainty into separate sources (described in Section 5.7.1) has been constructed to reproduce the correlation matrix obtained when deriving the uncertainty itself when each source is treated as fully correlated in both $p_{T}$ and rapidity.

JES: noise thresholds Given that the technique to form topoclusters is the same for all calorimeters and the calorimeter technologies are similar in the central region, this source is treated as fully correlated in $p_{T}$ and in $y$ in the central region $(y<0.8)$.

JES: theoretical uncertainty These sources are treated as fully correlated in $p_{T}$ and $y$ in the central region. This uncertainty source is most relevant at low $p_{T}$, and there it is assumed 


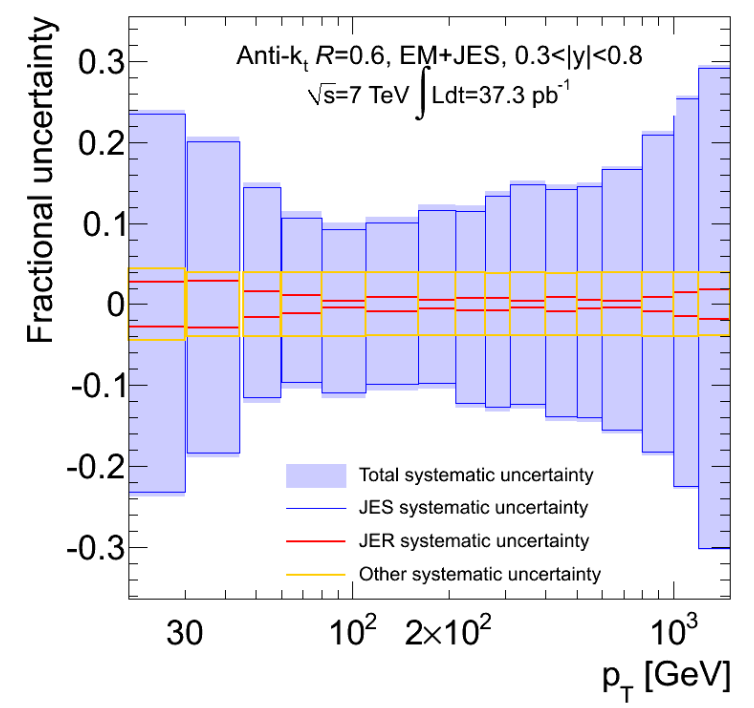

(a)

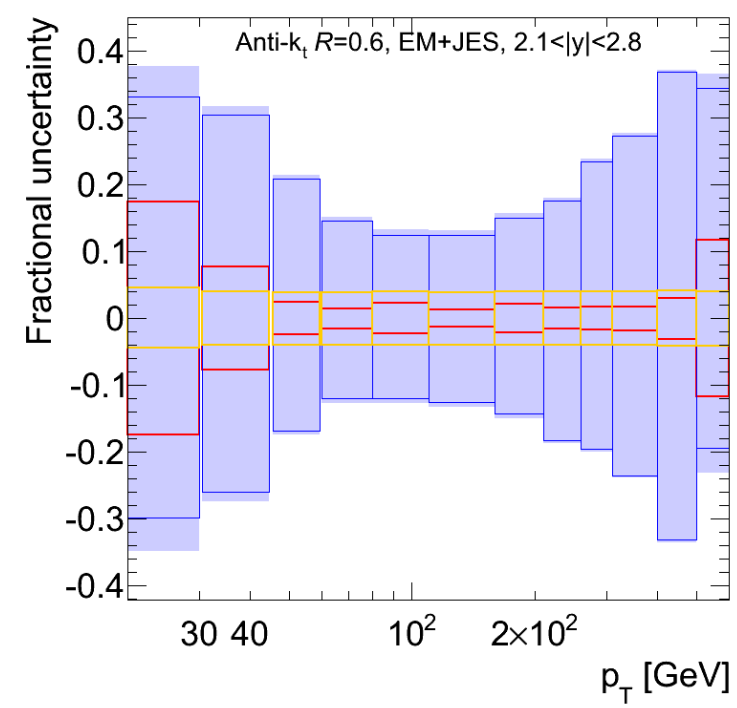

(b)

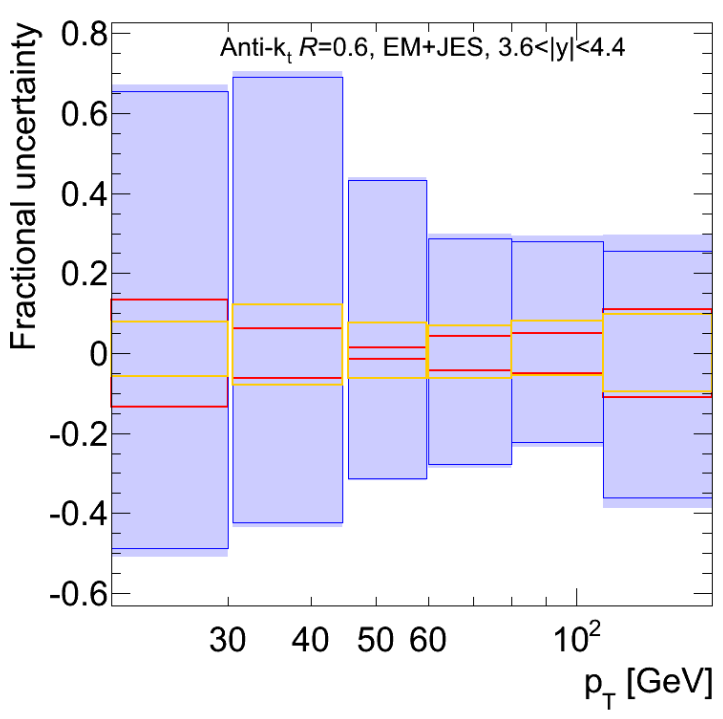

(c)

Figure 6.4.:Relative impact of the sources of systematic uncertainties on the inclusive jet cross section measurement for $R=0.6$ in three sample rapidity bins (central in Figure (a), endcap in Figure (b) and forward in Figure (c)).

that the different soft physics modeling of the Monte Carlo generators used will produce coherent shifts in the jet energy scale of neighboring bins.

JES: dead material The central region $(|y|<0.8)$ is composed of similar calorimeter technologies, therefore this source is treated as fully correlated in $p_{T}$ and $y$. 
All JES uncertainties used as a baseline The calorimeter uncertainty and the previous three sources (theoretical uncertainty, noise thresholds, dead material - not included in the calorimeter uncertainty) do not have a direct correspondence for rapidities above $y=0.8$ where they have been estimated. These sources will affect the bins above $y=0.8$ because the central region is used as reference in the intercalibration procedure employed to obtain the endcap and forward uncertainty. The correlations in $p_{T}$ need to be mantained within each of the rapidity regions, but there is no indication that the baseline uncertainty in different rapidity bins will be correlated, given that jets were not calibrated using the information in the intercalibration analysis and that different calorimeter technologies are employed to measure jets in the different rapidity bins.

JES: intercalibration since the largest contribution to the intercalibration uncertainty is that on the physics modelling of radiation in the forward region, assumed to vary coherently in $p_{T}$ and $\eta$, this source is treated as fully correlated in $p_{T}$ and $y$.

Calibration non closure This source is treated as fully correlated in $p_{T}$ but uncorrelated between central and forward $\eta$ bins (above $|y|<0.8$ ). This uncertainty component is derived separately for each $\eta$ bin (so it is not propagated from the central to the forward region). It is dominant at low $p_{T}$, where mass effects lead to non-closure in the calibration procedure that restores energy - for this reason neighboring bins of similar $p_{T}$ are considered fully correlated. Another source of non closure are effects due to poor resolution in the calibration procedure in difficult calorimeter regions (e.g. the $2.8 \leq|y|<3.6$ bin located in the transition between the HEC and the FCal) that lead to increased statistical fluctuations when deriving the calibration. If the mean energy at the electromagnetic scale is not well determined from a Gaussian fit, the constants will not be optimal leading to an imperfect closure. For this reason this source is considered uncorrelated between bins with different calorimeter technologies.

Unfolding uncertainty (closure test) This sources is treated as fully correlated in $p_{T}$ and $y$ bins. It is obtained through a closure test that connects coherently neighboring $p_{T}$ bins by varying the shape of the spectrum used for the unfolding. Each $y$ bin is unfolded independently but there is a similar trend observed in all $y$ regions when testing the closure of the method, and this justifies the rapidity correlation.

Jet matching uncertainty Since the matching uncertainty is derived applying the same change in matching radius for all $y$ and $p_{T}$ bins, it is considered fully correlated.

Jet energy resolution This source is treated as fully correlated in $p_{T}$. The consequence of the steepness of the jet spectrum is that the flux of jets fluctuating to higher $p_{T}$ bins 
dominates over jets fluctuating to lower $p_{T}$ bins. Therefore all bins in the spectrum will fluctuate coherently upwards if the resolution is worse than the one described in the Monte Carlo, as estimated with this uncertainty source. The resolution uncertainty is considered uncorrelated between central and forward $y$ bins, due to the different calorimeter technologies and increasing energies of jets at increasing pseudorapidities. The resolution uncertainty is fully correlated between the first two $y$ bins because the dijet balance technique used for the estimate of the resolution analyses them as a single $y$ region.

Angular resolution The angular resolution is influenced by the jet calibration, namely by the origin and $\eta$ correction. These have been derived in Monte Carlo from simulation of the different ATLAS calorimeter technologies, and it is therefore expected that the smearing of the jet position and its uncertainty will not be correlated between $y$ regions. This uncertainty is considered as fully correlated in $p_{T}$ because a shift in the jet position will have a coherent impact on the $p_{T}$ spectrum regardless of the jet transverse momentum.

Trigger This uncertainty source should be treated as completely correlated in $p_{T}$ and $y$ bins where the same jet trigger or threshold is used. Given that this is a small uncertainty source, that two different trigger systems are used for central and forward jets and that there are a number of trigger combinations almost as high as the number of bins used, it is approximated as completely uncorrelated for the analysis in this thesis.

Jet identification This uncertainty is treated as completely uncorrelated in $p_{T}$ and in $y$. The probability of a real jets flagged as fake by the different cuts depends on the jet $p_{T}$ : for example at very high $p_{T}$ calorimeter saturation could cause an abnormal calorimeter pulse shape that is then flagged as low quality. Furthermore, the systematic uncertainties on the cleaning include the statistical fluctuations given that the estimate of the jet identification efficiency and its uncertainties is performed on the same sample used for the cross section measurement. The efficiency of the cuts depends also on the calorimeter technology, and different values for the cuts are used in different rapidity regions, introducing $y$ decorrelations.

Reconstruction efficiency : This source is treated as completely correlated in $p_{T}$ and partially correlated in $y$. The decorrelation in $y$ reflects the different techniques used to estimate the reconstruction efficiency for $|\eta|<2.1$ (in-situ using track jets) and $|\eta|>2.1$ (using Monte Carlo truth jets). Correlation in $p_{T}$ is assumed given that a systematic underestimate of the reconstruction efficiency in data would affect all bins coherently.

Pile-up Given that the correlations in the extra jet energy due to additional soft physics interactions are on average removed by the pile-up offset correction and that the remaining 
pile-up uncertainty depends on effects that don't show obvious correlations in $y$ and $p_{T}$ (e.g. discrepancy with track jet validation, approximation on the average number of towers per jet), the pile-up uncertainty is considered as completely uncorrelated. Furthermore, any correlations introduced by potential bias affecting e.g. the number of primary vertices used to derive and apply the correction are mitigated by the mixing of different primary vertices and triggers used in the various $p_{T}$ and $y$ bins of the measurement.

Luminosity This source is treated as fully correlated in $p_{T}$ and $y$ since a change in the estimated luminosity will shift coherently all bins.

\subsection{Theoretical calculations for comparison to data}

After having been corrected for detector effects as explained in Section 6.3.1, the inclusive jet cross section measured in data is compared to the next to leading order theoretical prediction, which either is corrected for or includes ${ }^{4}$ non perturbative QCD effects.

The configuration used for the baseline theoretical prediction is the NLOJET ++4.1 .2 [32] software, which provides the next to leading order prediction for the inclusive jet cross section, together with the CT10 next to leading order PDF [151].

The renormalisation $\left(\mu_{R}\right)$ and factorisation $\left(\mu_{F}\right)$ scales (as introduced in Section 1.2) for the inclusive jet measurement are fixed to the transverse momentum of the jet.

Since partons are not observable physics objects, the next to leading order prediction needs to be corrected for non-perturbative effects the partons would be subject to (hadronisation, underlying event), in order to obtain a theoretical prediction that is comparable to data. The non-perturbative correction is obtained as a multiplicative factor in each bin of the theoretical prediction by turning off hadronisation ( $\mathrm{had}$ ) and underlying event and multiple parton interactions (denoted as $U E$ ) in a leading order + parton shower Monte Carlo generator. The correction is equal to the ratio of the Monte Carlo prediction for the number of jets with and without the non perturbative effects:

$$
C_{U E / h a d}=\frac{N_{j e t s}^{U E / h a d}}{N_{j e t s}^{n o U E / n o ~ h a d ~}},
$$

where the product of $C_{U E}$ and $C_{h a d}$ is taken to form the final multiplicative correction.

\footnotetext{
${ }^{4}$ The data has also been compared to POWHEG [150], a NLO ME generator that can include parton shower effects coherently employing other generators such as PYTHIAor HERWIG.
} 
Different non perturbative effects have different impact on narrower or wider jets:

- The correction for low- $p_{T}$ anti- $k_{t} R=0.4$ jets is dominated by the effect of hadronisation particles falling outside the jet. It becomes unity at high $p_{\mathrm{T}}^{\text {jet }}$, where the hadronized daughters of the boosted parton will be more collimated. For anti- $k_{t} R=0.6$ jets the correction is less important, and still goes to unity at high transverse momentum.

- The correction for low- $p_{T}$ anti- $k_{t} R=0.6$ jets is dominated by the effect of the underlying event adding particles to the jet. Its importance decreases with transverse momentum since underlying event effects are to first order uncorrelated to the hard scatter.

The non perturbative corrections are derived in bins of $y$ using the newest tune to the 2010 ATLAS data for the PYTHIA generator (called AUET2B and described in [152]), using the CTEQ6L1 PDF [43]. For low- $p_{T}$ anti- $k_{t} R=0.6$ jets the total correction can reach values of 1.6 at low $p_{T}$ in the central rapidity regions, while for anti- $k_{t} R=0.4$ jets the largest deviation of the correction from unity $(0.9)$ is found at low- $p_{T}$ in the forward region. Since the estimate of these corrections enters PDF fits when comparing data to theory in the $\chi^{2}$ minimisation (as explained in the next chapter), the appropriate uncertainties should be considered when using theoretical prediction where the NLO cross section is mostly influenced by non-perturbative physics.

\subsubsection{Theoretical uncertainties and uncertainties on non perturbative corrections}

In order to compare the theoretical prediction with the measurement, the theoretical uncertainties due to the choices made in the NLO calculation, the PDF convolution and in the derivation of the non perturbative correction need to be evaluated.

The uncertainties on the theoretical prediction are due to the systematic uncertainties on the parton distribution function used for the convolution, to the renormalisation and factorisation scales and to the value of the strong coupling constant $\alpha_{s}$ used for the QCD evolution. In order to reduce the time necessary to evaluate the effect of the systematic uncertainties, the APPLGRID [33] software is interfaced with NLOJET++ as described in Section 1.4.

The theoretical uncertainties are estimated as follows and added in quadrature to form the total theoretical uncertainty: 
Renormalisation and factorisation scales : the sensitivity to the pQCD scales is evaluated by varying the two values independently by twice and half their nominal value ${ }^{5}$ and re-evaluating the theoretical prediction using each combination. The envelope of all variations is taken as systematic uncertainty, and it amounts to less than $5 \%$, with the largest uncertainties at low $p_{T}$ for anti- $k_{t} R=0.6$.

Uncertainties on the chosen PDF : the inclusive jet cross section is calculated using the parameters corresponding to the positive and negative variations of the eigenvectors of the Hessian matrix of the CT10 PDF fit (as detailed in Section 7.2) The uncertainty is estimated as the deviation of the predictions with the systematically shifted parameters with respect to the nominal theoretical prediction, and normalised to a one-sigma confidence estimate $^{6}$. The total PDF uncertainty amounts to $3 \%$ at low $p_{\mathrm{T}}^{\text {jet }}$ and increases with transverse momentum until it dominates the theoretical uncertainty at $\approx 200 \mathrm{GeV}$.

Strong coupling constant : following the CTEQ prescription [153], the cross section is reevaluated using special PDF sets with $\alpha_{s}$ values that are varied within its best estimate. The theoretical prediction is then compared to that obtained with the central PDF set (with the nominal value of $\alpha_{s}$ ). This uncertainty, together with the baseline PDF uncertainty, covers the full effect of the variation of the strong coupling constant. The uncertainty due to $\alpha_{s}$ is $2-3 \%$ for low $p_{\mathrm{T}}^{\text {jet }}$ and increases to $4-5 \%$.

Non perturbative corrections : The uncertainty on the theoretical prediction is calculated by estimating the effect of non perturbative effects using different tunes and generators. Alternative non perturbative correction factors are estimated using a variety of tunes for both Pythia 6.5, its newest version Pythia 8.150) and for Herwig++ 2.5.1. These generators have been tuned to ATLAS data and are described in Reference [154]. The PYTHIA 6 tunes employed are the AMBT2B tune with the CTEQ6L1 PDF set, the AUET2 tune which uses the modified leading order PDF LO from the MRST collaboration [155] and the AMBT1 tune (with the MRST LO* PDF) that corresponds to that used for the nominal sample described in Section. The UE7000-2 tune is employed for the HERWIG++ generator, while the tune used for PYTHIA 8 is called 4C. The maximum spread of the alternative correction factors around the nominal correction is taken as systematic uncertainty on the theoretical prediction. This uncertainty dominates the theoretical uncertainty wherever the correction is larger and driven by the differences in the soft

\footnotetext{
${ }^{5}$ The two scales are not both varied in opposite directions to avoid the unphysical situation in which large logarithms appear in the calculation.

${ }^{6}$ The values provided by the CTEQ collaboration correspond to a $90 \%$ confidence level interval.
} 
physics models of the various tunes and generators: it amounts to up to $26 \%$ for anti- $k_{t}$ $R=0.6$ and up to $10 \%$ for $R=0.4$.

\subsection{Experimental Results and comparison to theory}

Figure 6.5 shows the measured inclusive jet cross section and the theoretical prediction as a function of the jet $p_{T}$ in the different rapidity bins. The cross section for jets with distance parameter $R=0.4$ is shown in Figure 6.5 (a), while the result for jets with $R=0.6$ is shown in Figure 6.5 (b). Both measurement and predictions have been scaled by a factor to enhance the legibility of the plot. The ratio of the data to the theoretical prediction is displayed in Figures 6.6 and 6.7 for $R=0.4$, and in Figures 6.8 and 6.9 for $R=0.6$. The kinematic range of the measurement spans more than $1 \mathrm{TeV}$ in the central bins, and the jet cross sections falls by more than seven orders of magnitude in this range. The data and theoretical prediction are in agreement within uncertainties for the whole kinematic range. Some tension can be seen in the two forwardmost bin, where a trend for data to be lower than the theoretical prediction is present in the highest $p_{T}$ bins. However, the systematic uncertainties in both data and theory are too large to be able to discern any significant effect, and to use this specific information as input to theory. The cross section results after all corrections, the theoretical prediction and the breakdown of the theoretical and experimental uncertainties for three sample rapidity regions can also be found in Appendix E, while the full set of results will be made available on HEPData [156]. 


\begin{tabular}{|l|c|c|c|c|c|c|c|}
\hline & \multicolumn{7}{|c|}{$|y|$-bins } \\
Uncertainty Source & $0-0.3$ & $0.3-0.8$ & $0.8-1.2$ & $1.2-2.1$ & $2.1-2.8$ & $2.8-3.6$ & $3.6-4.4$ \\
\hline Noise thresholds & 1 & 1 & 2 & 3 & 4 & 5 & 6 \\
\hline PYTHIA Perugia2010 & 7 & 7 & 8 & 9 & 10 & 11 & 12 \\
\hline AlPGEN+HERWIG+JIMMY & 13 & 13 & 14 & 15 & 16 & 17 & 18 \\
\hline JES calibration non closure & 19 & 19 & 20 & 21 & 22 & 23 & 24 \\
\hline Additional dead material & 25 & 25 & 26 & 27 & 28 & 29 & 30 \\
\hline Intercalibration & 31 & 31 & 31 & 31 & 31 & 31 & 31 \\
\hline Calorimeter: E/p & 32 & 32 & 33 & 34 & 35 & 36 & 37 \\
\hline Calorimeter: Cluster thresholds & 38 & 38 & 39 & 40 & 41 & 42 & 43 \\
\hline Calorimeter: LAr E-scale & 44 & 44 & 45 & 46 & 47 & 48 & 49 \\
\hline Calorimeter: Tile E-scale & 50 & 50 & 51 & 52 & 53 & 54 & 55 \\
\hline Calorimeter: CTB High $p_{T}$ & 56 & 56 & 57 & 58 & 59 & 60 & 61 \\
\hline Calorimeter: E/p bias & 62 & 62 & 63 & 64 & 65 & 66 & 67 \\
\hline Calorimeter: CTB bias & 68 & 68 & 69 & 70 & 71 & 72 & 73 \\
\hline Unfolding: closure test & 74 & 74 & 74 & 74 & 74 & 74 & 74 \\
\hline Unfolding: truth matching & 75 & 75 & 75 & 75 & 75 & 75 & 75 \\
\hline Jet energy resolution & 76 & 76 & 77 & 78 & 79 & 80 & 81 \\
\hline Angular resolution & 82 & 82 & 82 & 82 & 82 & 82 & 82 \\
\hline Trigger modelling & uncorr & uncorr & uncorr & uncorr & uncorr & uncorr & uncorr \\
\hline Jet cleaning & uncorr & uncorr & uncorr & uncorr & uncorr & uncorr & uncorr \\
\hline Jet reconstruction efficiency & 83 & 83 & 83 & 83 & 84 & 85 & 86 \\
\hline Pile-up & uncorr & uncorr & uncorr & uncorr & uncorr & uncorr & uncorr \\
\hline Luminosity & 87 & 87 & 87 & 87 & 87 & 87 & 87 \\
\hline
\end{tabular}

Table 6.1.:Schema of the bin-to-bin uncertainty correlations between $|y|$-bins for the inclusive jet measurement. Each number (each number also corresponds to a different color) represents a separate nuisance parameter to be used in fits. Bins with the same nuisance parameter are treated as fully correlated, while bins with different nuisance parameters are considered uncorrelated. All uncertainty sources listed here are fully correlated versus $p_{\mathrm{T}}$. The uncertainties marked as uncorr are treated as uncorrelated. 


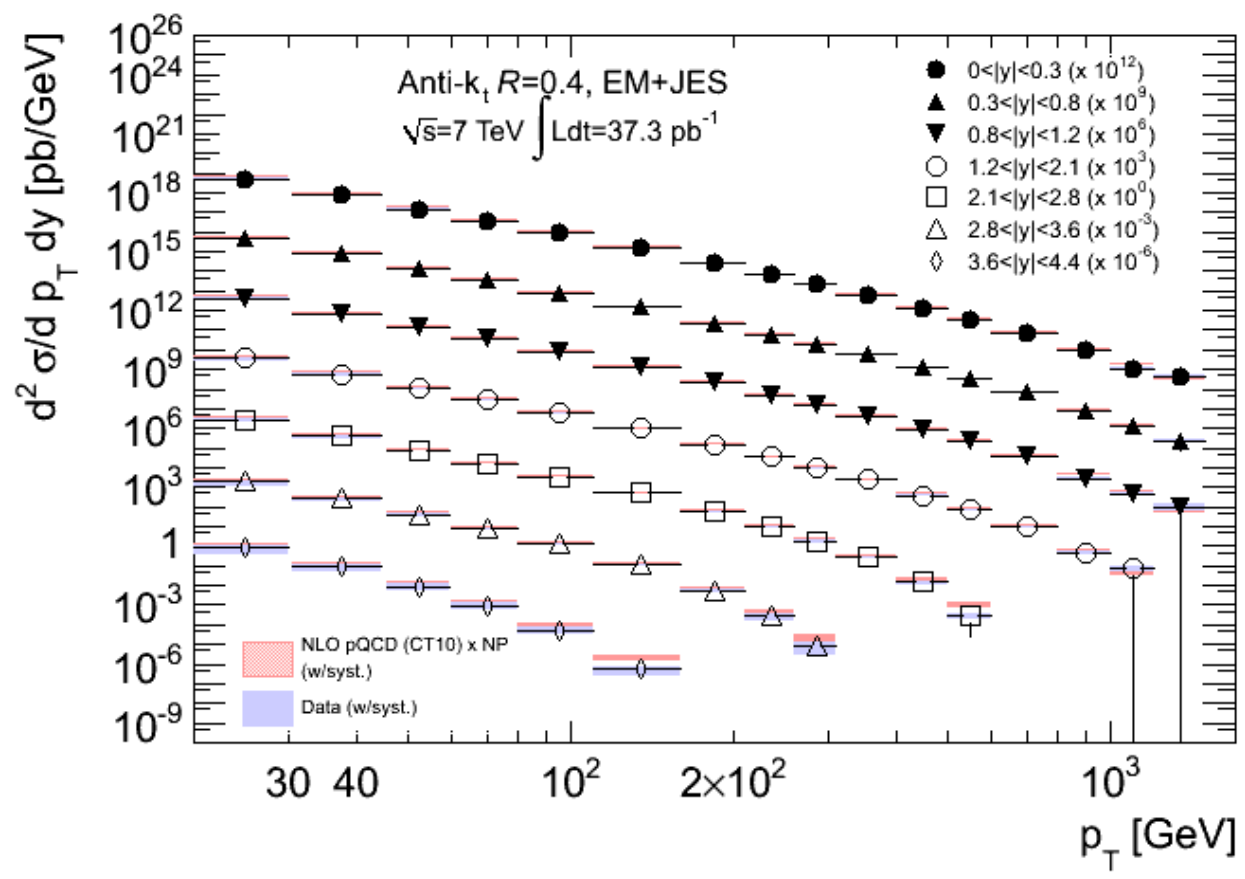

(a)

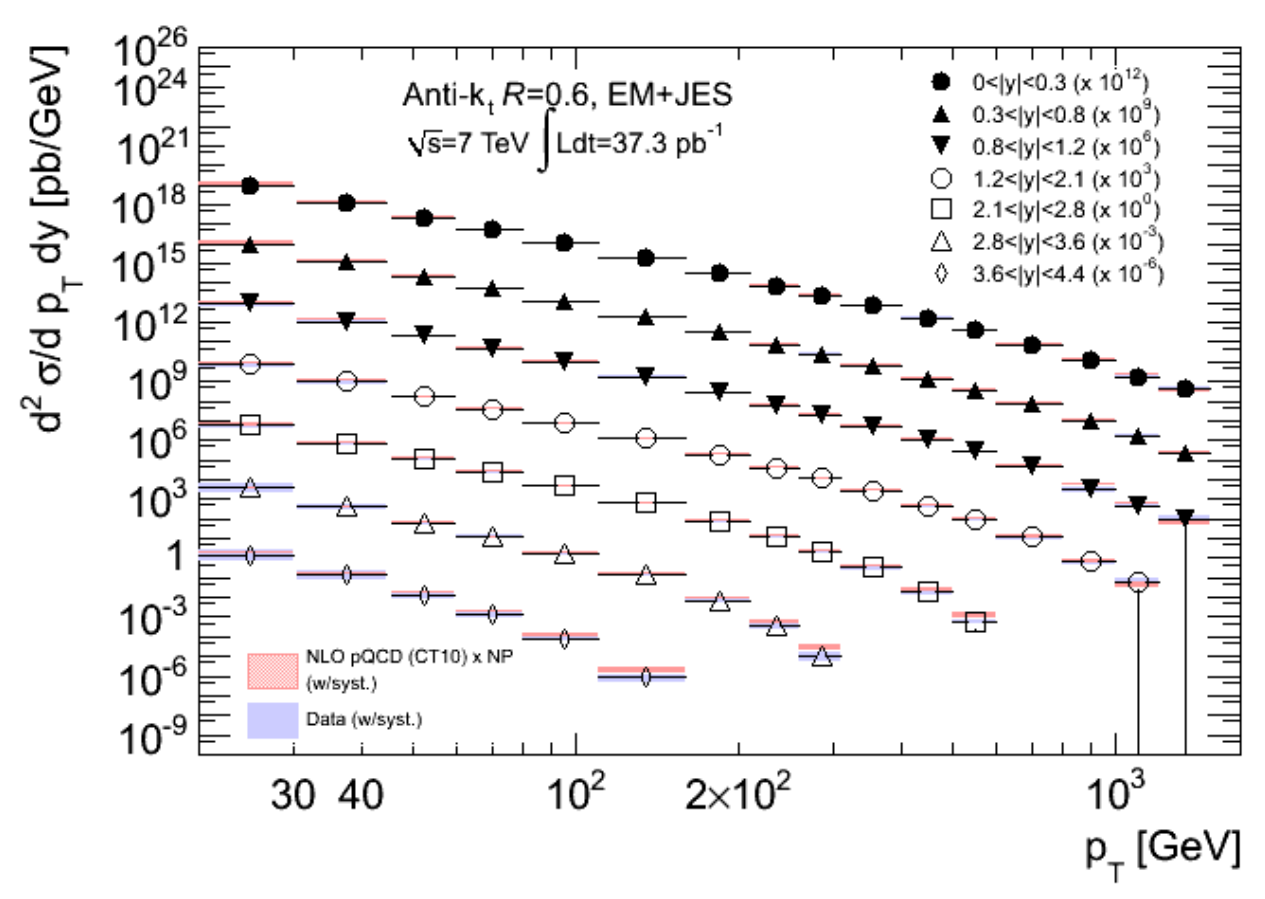

(b)

Figure 6.5.:Inclusive jet double-differential cross section as a function of jet $p_{T}$ in different $|y|$ bins for anti- $k_{t} R=0.4$ jets (a) and anti- $k_{t} R=0.6$ jets (b). The statistical uncertainties are shown on the data points as error bar, while the total systematic uncertainty are shown as the shaded blue band around the data points. The luminosity uncertainty of $3.4 \%$ is not shown. The total uncertainty on the theoretical prediction (due to non-perturbative corrections, PDFs, variations of the strong coupling constant and factorisation/renormalisation scales) is shown as the shaded pink band, centered on the theoretical prediction derived using the CT10 PDF and corrected for 1229 perturbative effects. 


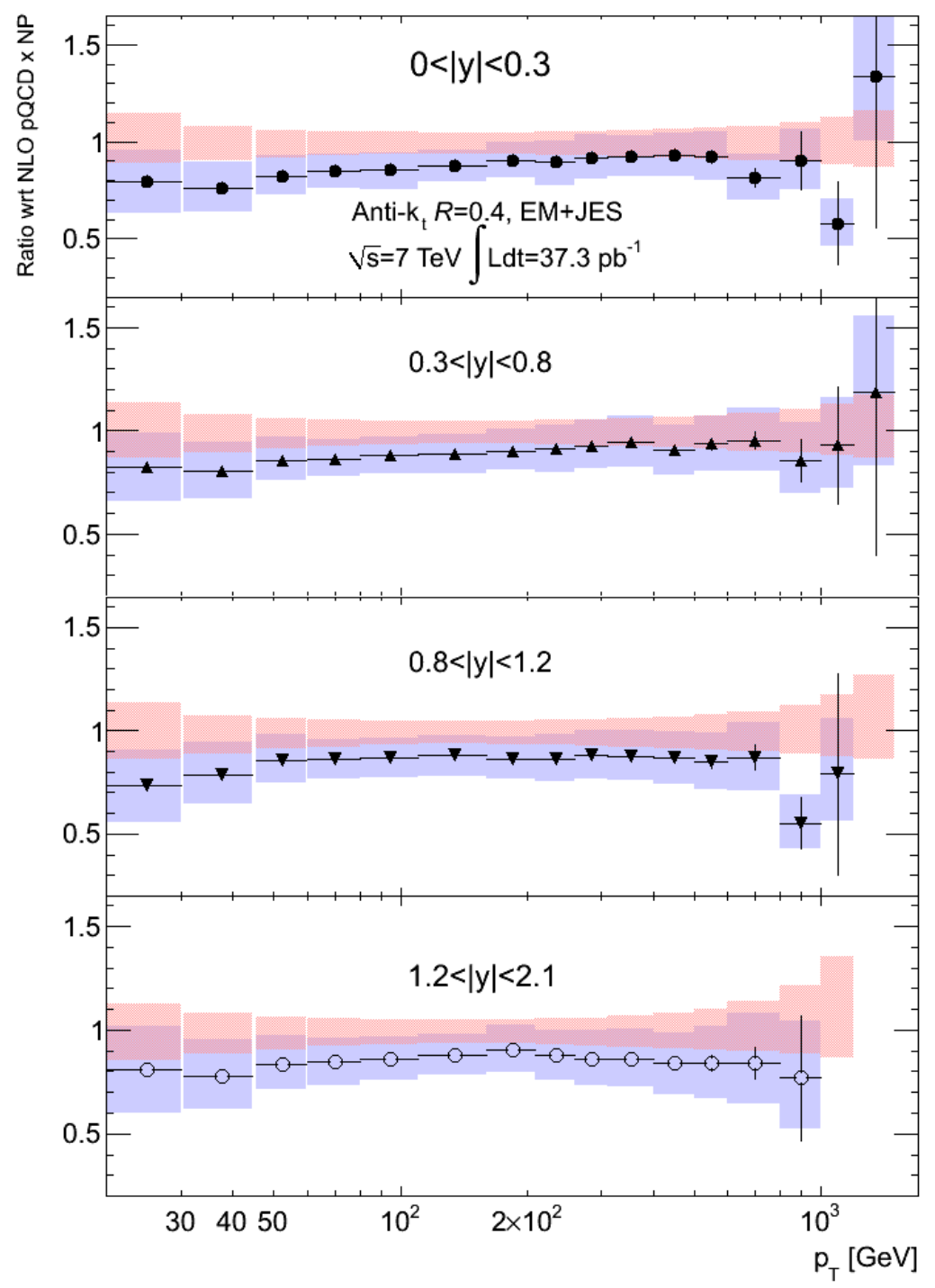

Relative systematic uncertainty on NLO pQCD (CT10) x NP

Data, ratio wrt NLO pQCD (CT10) x NP (with stat./syst. uncertainty)

Figure 6.6.:Ratio of the inclusive jet double-differential cross section measured in data with respect to the NLO pQCD calculation using the CT10 PDF with non-perturbative corrections applied, as a function of jet $p_{T}$ in $|y|$ bins $(|y|<1.2)$ for anti- $k_{t} R=0.4$ jets. The statistical uncertainty on the data/theory ratio is shown as the error on the data point, while the systematic uncertainty on data is the shaded blue band. The total uncertainty on the theoretical prediction (due to non-perturbative corrections, PDFs, variations of the strong coupling constant and factorisation/renormalisation scales) is shown as the shaded pink band, centered on unity. The luminosity uncertainty of $3.4 \%$ is not shown. 


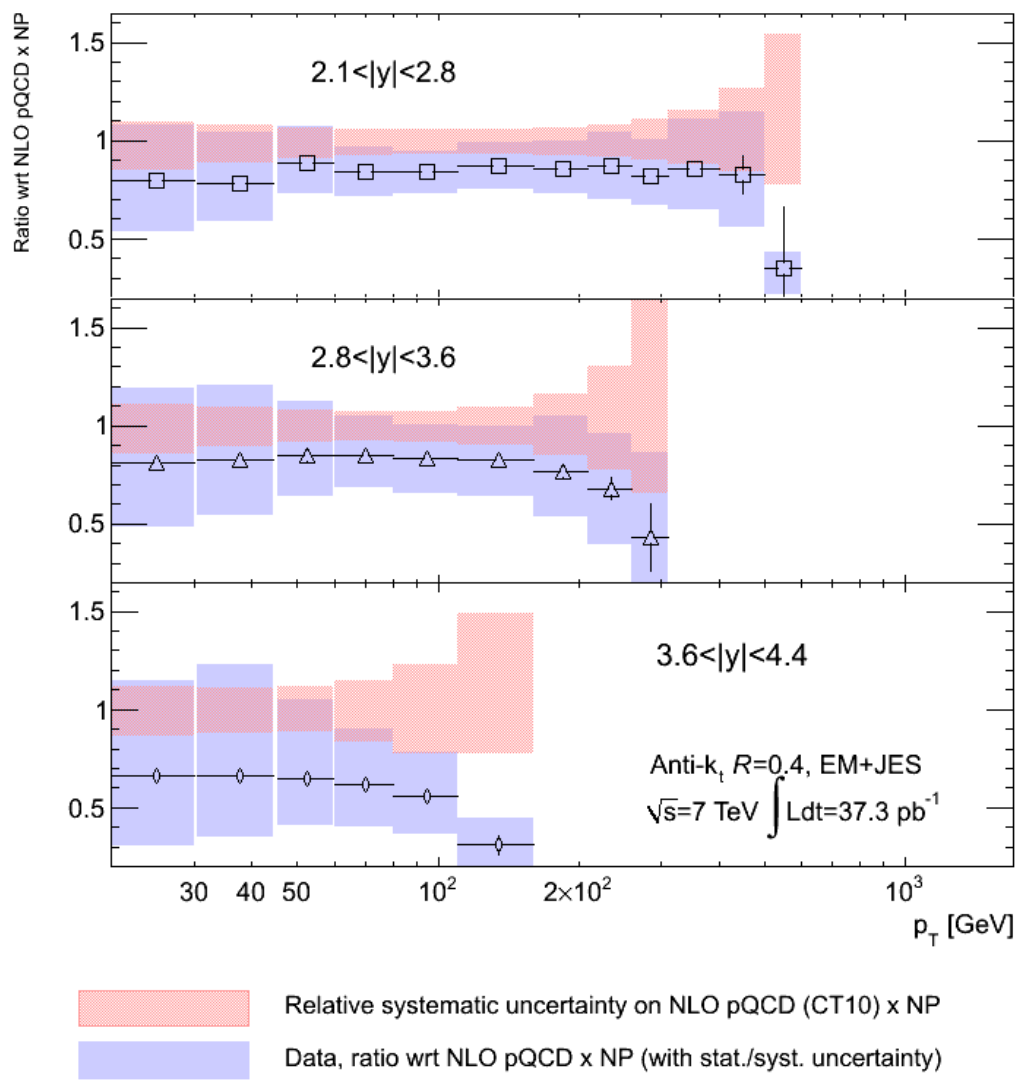

Figure 6.7.:Ratio of the inclusive jet double-differential cross section measured in data with respect to the NLO pQCD calculation using the CT10 PDF with non-perturbative corrections as a function of jet $p_{T}$ in $|y|$ bins $(1.2<|y|<4.4)$ for anti- $k_{t} R=0.4$ jets. The statistical uncertainty on the data/theory ratio is shown as the error on the data point, while the systematic uncertainty on data is the shaded blue band. The total uncertainty on the theoretical prediction (due to non-perturbative corrections, PDFs, variations of the strong coupling constant and factorisation/renormalisation scales) is shown as the shaded pink band, centered on unity. The luminosity uncertainty of $3.4 \%$ is not shown. 


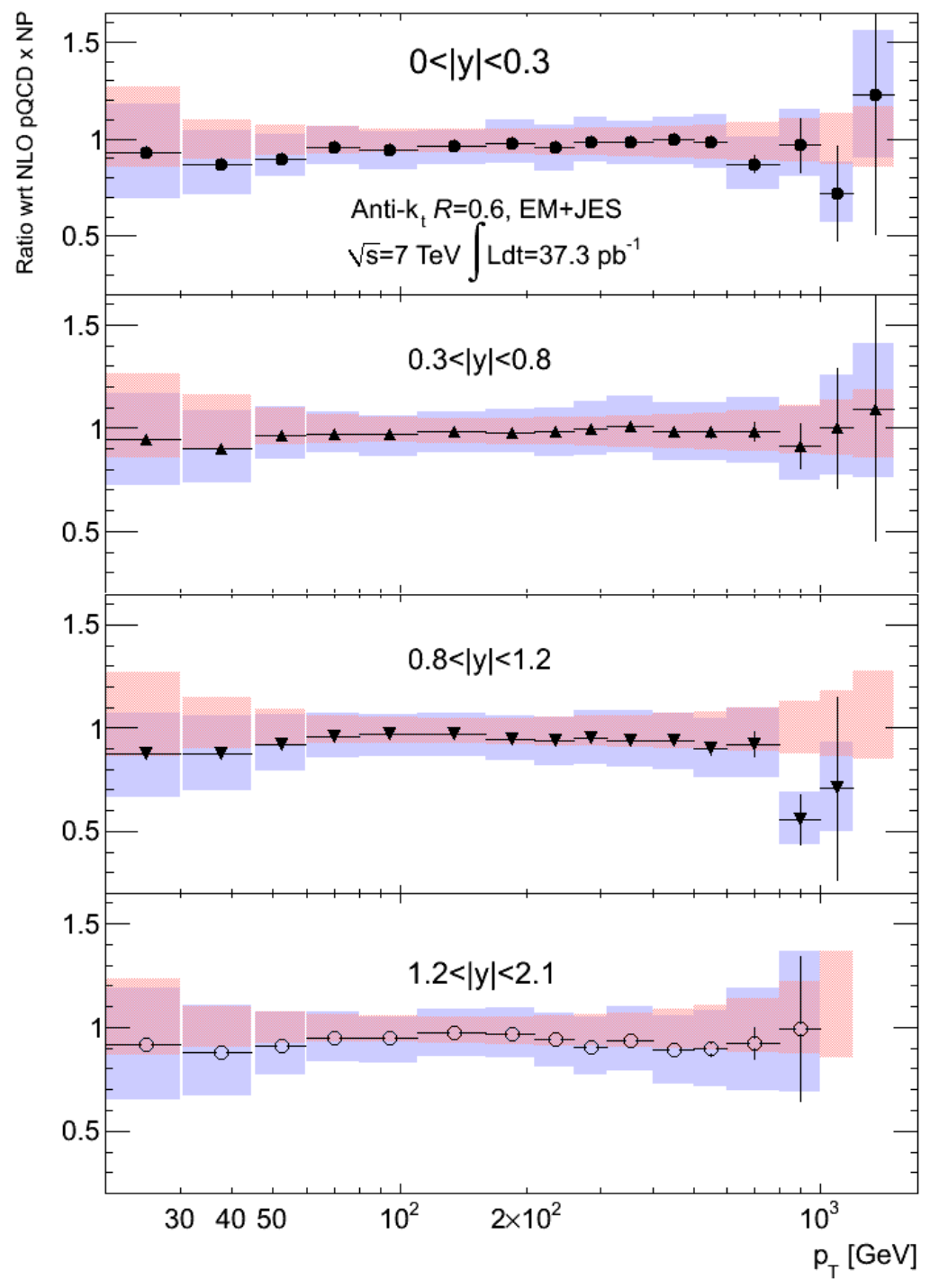

Relative systematic uncertainty on NLO pQCD (CT10) x NP

Data, ratio wrt NLO pQCD (CT10) x NP (with stat./syst. uncertainty)

Figure 6.8.:Ratio of the inclusive jet double-differential cross section measured in data with respect to the NLO pQCD calculation using the CT10 PDF with non-perturbative corrections as a function of jet $p_{T}$ in $|y|$ bins $(|y|<1.2)$ for anti- $k_{t} R=0.6$ jets. The statistical uncertainty on the data/theory ratio is shown as the error on the data point, while the systematic uncertainty on data is the shaded blue band. The total uncertainty on the theoretical prediction (due to non-perturbative corrections, PDFs, variations of the strong coupling constant and factorisation/renormalisation scales) is shown as the shaded pink band, centered on unity. The luminosity uncertainty of $3.4 \%$ is not shown. 


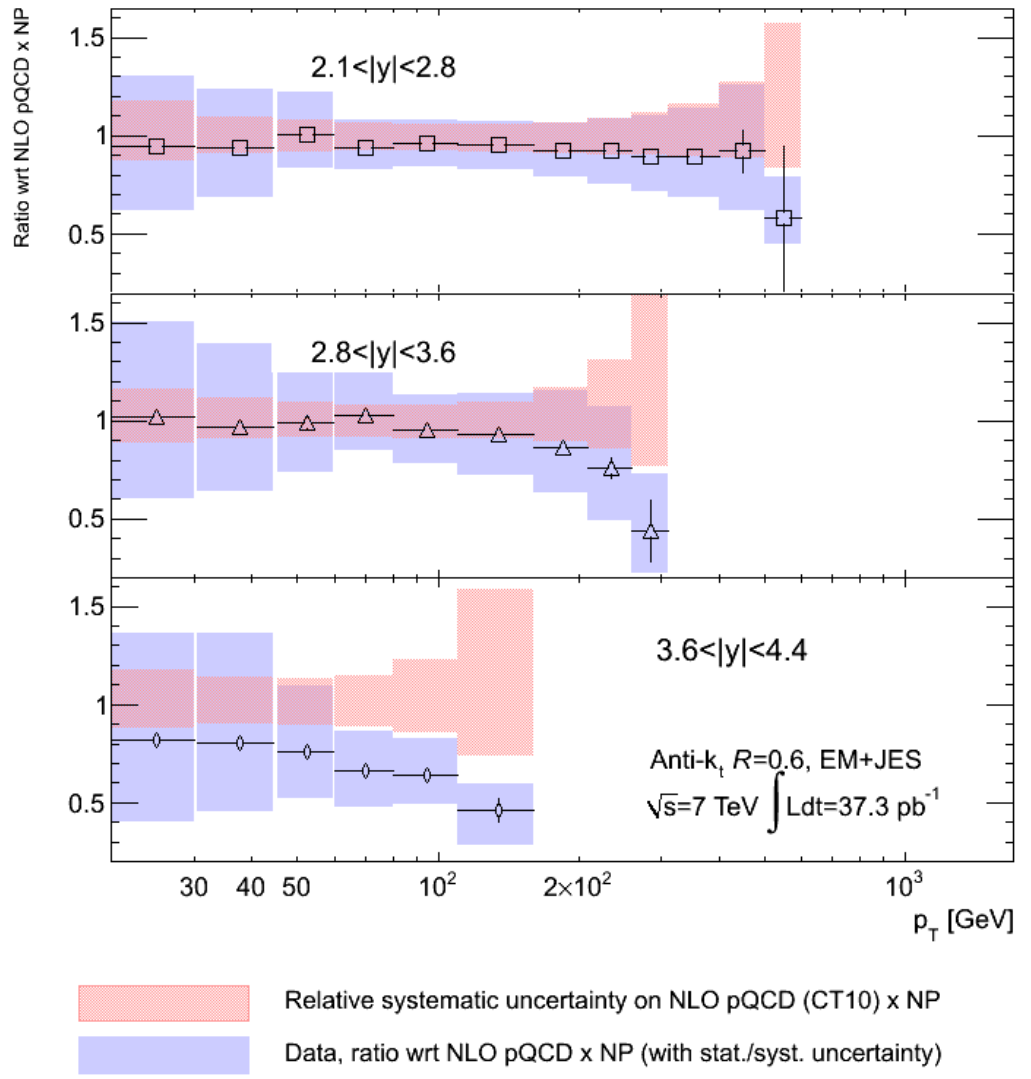

Figure 6.9.:Ratio of the inclusive jet double-differential cross section measured in data with respect to the NLO pQCD calculation using the CT10 PDF with non-perturbative corrections as a function of jet $p_{T}$ in $|y|$ bins $(|y|<1.2)$ for anti- $k_{t} R=0.6$ jets. The statistical uncertainty on the data/theory ratio is shown as the error on the data point, while the systematic uncertainty on data is the shaded blue band. The total uncertainty on the theoretical prediction (due to non-perturbative corrections, PDFs, variations of the strong coupling constant and factorisation/renormalisation scales) is shown as the shaded pink band, centered on unity. The luminosity uncertainty of $3.4 \%$ is not shown. 


\section{Chapter 7.}

\section{Impact of ATLAS jet data on PDF fits}

The knowledge of parton distribution functions is a fundamental input to theoretical predictions in hadronic collisions. The uncertainty on the PDFs feeds into the theoretical uncertainty on the inclusive jet cross section, as shown in the previous chapter. It also impacts new physics measurements in terms of e.g. the QCD background model used and expected production cross sections.

It is therefore important to use all available data to constrain the PDFs and minimise their uncertainties. Measurements of electroweak and QCD physics at the LHC span a kinematic region in the $Q^{2}$ and $x$ plane that was not yet covered by previous experiments whose data was used as input to PDF fits, as shown in Figures 7.1 (a) and (b). The inclusive jet cross section measurement span an approximate $x$ range of $7 \cdot 10^{5}<x<0.8$, and the highest value for the dijet mass with the current data is approximately $5 \mathrm{TeV}$.

The knowledge of the gluon PDF is of particular relevance since gluon-gluon scattering dominates at the Large Hadron Collider. The gluon PDF can be constrained in deep inelastic scattering measurements by using scaling violations as mentioned in Section 1.2.2, and by using jet data in DIS for values of $x$ from $\approx 0.01$ to 0.1 . DIS data therefore do not provide much information at high $x$. Furthermore, the only sum rule that constrains the gluon distribution in deep inelastic scattering is the momentum sum rule mentioned in Section 1.2.2. Using only this sum rule to constrain the gluon PDF would require an integral over the experimentally unmeasurable $x$ extremes of 0 and 1, together with a very good knowledge of all the other quark and antiquark distributions. For these reason QCD processes where gluons directly enter the non perturbative evolution at leading order (such as the inclusive jet cross section measurement) are an important input to improve the gluon PDF. 


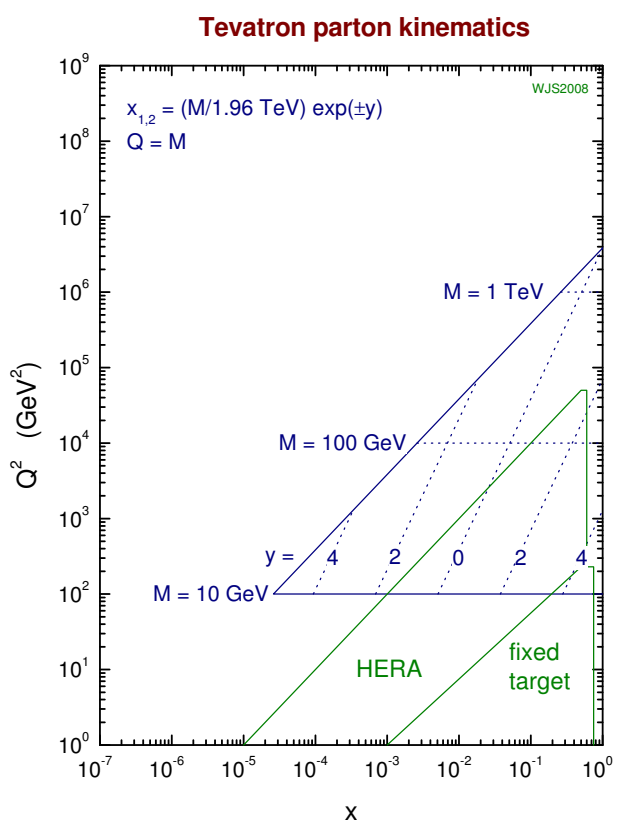

(a)

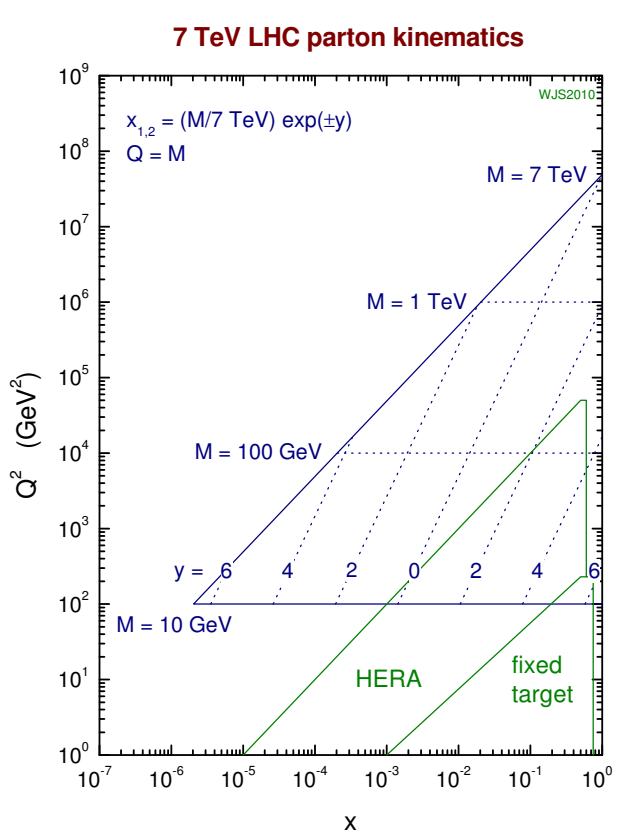

(b)

Figure 7.1.:Parton kinematics in the $x, Q^{2}$ plane for LHC and Tevatron colliders. The kinematic range of the HERA collider and fixed target experiments that are used as input to PDF fits is also included for comparison. In the figure, $M$ indicates the mass of a given heavy particle produced at a rapidity $y$. Figures from [157].

The aim of the study in this chapter is to evaluate the impact of ATLAS jet data on the PDF parameters and their uncertainties when included in a PDF fit that uses data from the HERA collider [158] as a baseline. These results need to be taken as a proof of principle that ATLAS data can have a non-negligible impact on PDF fits even though the dominant JES uncertainty has not yet reached the desired $1 \%$ precision. They will also serve as a stepping stone for future work given that a more detailed knowledge on the fitting techniques, treatment of correlated uncertainties and on the details of the code has been reached through this work.

This chapter reviews in more detail the procedure to extract the parton density functions from experimental data (Section 7.1), describing the strategy for PDF fits. The treatment of experimental errors and the propagation of the uncertainties from the PDF parameters to observables is outlined in Section 7.2. The HERA PDF parametrisation and fitting method are described in more detail in Section 7.3. Finally, the effect of including ATLAS inclusive jet cross section data in PDF fits together with the HERA data is shown in Section 7.4. 


\subsection{Extraction of parton distribution functions}

Parton distribution functions cannot be predicted from first principles in QCD, but they can be extracted from the comparison of experimental measurements and theoretical predictions as detailed in the following.

As a preliminary step to the fitting procedure, an arbitrary input shape for the gluon and quark PDF is needed at a starting scale $Q_{0}^{2}$ (again arbitrarily chosen). The choice of the basic input shape for the gluon and quark PDF has been described in Section 1.2.2, but usually more flexibility is given by adding an extra multiplicative polynomial term $P(x, f)$ to Equation 1.4:

$$
x f(x)=A x^{B}(1-x)^{C} P(x, f)
$$

Most experimental measurements do not have a high enough $Q^{2}$ to probe the top quark scale in the proton, and the top PDF is therefore neglected in PDF fits. Therefore, PDFs for 11 partons need to be extracted: 10 for the different flavours of quarks and antiquarks plus one for the gluon. However the charm and bottom PDFs can be calculated in perturbative QCD taking into account their mass and their production thresholds ${ }^{1}$ so this leaves a total of 7 PDFs, each with its parameters, to be determined. The choice of parametrisation and which parameters are left free in the fit varies between the different PDF fits, and they are outlined in the case of the HERAPDF fit in the following section.

Once the initial parametrisation at the starting scale has been chosen, the DGLAP equations are used to evolve the PDFs to the $Q^{2}$ scale of the measurement. Specific software packages (e.g. APPLGRID in the case of the inclusive jet cross section) are used to convolve the PDFs with the QCD coefficient functions, as outlined in Section 1.2. If needed (as in the case of electroweak/DIS measurements), the PDFs are then combined into the appropriate structure functions to form the full theoretical prediction. Minimisation of the $\chi^{2}$ built from the comparison of theoretical predictions to experimental data allows the extraction of the best fit PDF parameters. The explicit $\chi^{2}$ definition and techniques to handle experimental uncertainties and provide uncertainties on the PDF parameters are given in Section 7.2.

\footnotetext{
${ }^{1}$ This is generally done exploiting external programs.
} 


\subsubsection{PDF fits}

The large (10-20) number of parameters needed for a full determination of the chosen PDF combination require constraints from a number of different sources. Different processes can be combined in PDF fits, with each of the processes contributing to constraining a PDF or a combination of PDFs. A recent review of data included in PDF fits can be found in Reference [159]. Sets of PDFs are used as input to theoretical predictions, and they are updated regularly whenever new data is available. PDF sets that have been used in the ATLAS inclusive jet cross section measurement are MSTW08 [38, 160], CTEQ [43] and CT10 [151], NNPDF2.1 [161] and HERAPDF1.5 [158]. Comparison of theoretical predictions for the inclusive jet cross section using other available PDF sets can be found in Reference [162].

\subsection{Treatment of uncertainties}

Uncertainties on the fitted PDF parameters can be propagated to the PDFs themselves and thus to predictions for the measurable structure functions and cross sections. The main sources of uncertainty on PDF parameters are:

Experimental statistical errors This uncertainty can generally be considered uncorrelated among the various data points ${ }^{2}$.

Experimental systematic uncertainties Bin-to-bin correlations of the systematic uncertainties need to be treated in the fit, as detailed in Section 7.2.1.

Model and parametrisation uncertainties These uncertainties orginate from model assumptions and the choice of parametrisation at the starting scale.

Theoretical uncertainties These uncertainties follow from the use of the DGLAP framework and include the dependence on renormalisation/factorisation scale for higher order corrections and uncertainties on the non-perturbative corrections that need to be applied for the comparison to data.

Systematic uncertainties often do not have a well-defined one-sigma interpretation, since they might involve assumptions and they can be affected by limited knowledge of the experimenters. The question then arises whether estimating the parameter error boundaries using a variation

\footnotetext{
${ }^{2}$ This assumption does not necessarily hold in the inclusive jet cross section, where jets in different bins come from the same event. Even though correlations can be relevant in bins with low statistics (as in the case of high $p_{T}$ /high rapidity bins) and have been estimated after the unfolding procedure, the treatment of correlated statistical uncertainties in PDF fits is left for future studies.
} 
$\Delta \chi^{2}$ of unity around the minimum is sufficient to cover one-sigma systematic uncertainties on the PDF, since this assumes Gaussian errors with their correlations correctly treated. Furthermore, the inclusion of many different data sets in PDF fits can lead to large values of the fitted $\chi^{2}$ due to marginal compatibility of different data sets. In order to ensure that the uncertainties on the PDF parameters are not underestimated, some PDF fits use a so-called $\chi^{2}$ tolerance, and increase the $\Delta \chi^{2}$ used for the evaluation of errors to values that can be up to 40-50 (these figures are largely based on experience and vary depending on the PDF analysis).

Another issue that is present in the propagation of PDF errors to experimental quantities is that the errors on the PDF fit parameters are correlated since the error matrix for the PDF fit parameters is not diagonal (this follows from the correlations between the PDF parameters themselves). The Hessian matrix ${ }^{3}$ can be diagonalised and its eigenvectors are used for the propagation of the errors on the PDF parameters to the measurement. Each eigenvector will correspond to a combination of parameters, and the eigenvalue to the square of the error on this combination. For each eigenvalue, two sets of PDFs are provided, accounting for the upwards and downward shift of the chosen $\chi^{2}$ tolerance on the corresponding eigenvector. The error on a physical quantity $X$ can then be calculated using the following prescription (in the case of asymmetric errors):

$$
\begin{aligned}
\Delta X^{+} & =\sqrt{\sum_{i=1}^{N_{\text {vect }}}\left[\max \left(X_{i}^{+}-X_{0}, X_{i}^{-}-X_{0}, 0\right)\right]^{2}} \\
\Delta X^{-} & =\sqrt{\sum_{i=1}^{N_{\text {vect }}}\left[\max \left(X_{0}-X_{i}^{+}, X_{0}-X_{i}^{-}, 0\right)\right]^{2}},
\end{aligned}
$$

where $X$ is calculated using the PDF set corresponding to the upwards shifted $\left(X^{+}\right)$, downwards shifted $\left(X^{+}\right)$and central $X^{0} \mathrm{PDF}$ sets and $i$ is an index that runs on the $N_{\text {vect }}$ eigenvectors.

\subsubsection{Treatment of correlated experimental errors in PDF fitting}

When the dominant uncertainties in a measurement are the experimental systematic uncertainties, as in the case of the inclusive jet cross section measurement detailed in Chapter 6, it is

\footnotetext{
${ }^{3}$ The Hessian matrix represents the error on a measurement $X$ due to the different parameters $i$ and $j$ $\left(\sigma_{X}^{2}=\left(\partial_{i} \partial_{j} \chi_{\min }^{2}\right)\left(\partial_{i} \partial_{j} X\right)\right.$, where $\partial_{k}$ represents the partial derivative of a quantity with respect to the $k$-th PDF parameter. The underlying assumption that linear error propagation holds is made).
} 
particularly important to treat the correlated systematic errors correctly in fits that make use of the data/theory comparison to extract QCD parameters. In this study, the prescription of References $[43,163,164]$ is used and summarised below. The treatment of theoretical errors also requires care for a correct inclusion in the fits, but it is outside the scope of the study performed in this thesis, and theoretical errors are not accounted for in the following.

The simplest $\chi^{2}$ that can be used (in the case of a single experiment providing the $N$ data points $D_{i}$ ) neglects the correlations between systematic errors and is of the form:

$$
\chi^{2}(\mathbf{p})=\sum_{i=1}^{N} \frac{\left(D_{i}-T_{i}(\mathbf{p})\right)^{2}}{\sigma_{i, \text { stat }}^{2}+\sigma_{i, \text { uncorr }}^{2}}
$$

where $T_{i}$ is the point to point theoretical prediction that depends on the minimisation parameters $\mathbf{p}, \sigma_{i, \text { stat }}$ and $\sigma_{i, \text { uncorr }}$ the statistical and experimental errors respectively, considered as uncorrelated between the different data points. In a more realistic case, each of the data points can have $\mathrm{K}$ sources of correlated systematic errors $\beta_{k}$. The effect of correlated systematic errors can be parametrised using a series of parameters $r_{k}$ that are extracted from a Gaussian distribution with zero mean and unit width, and included in the fit as nuisance parameters:

$$
\chi^{2}(\mathbf{p}, \mathbf{r})=\sum_{i=1}^{N} \frac{1}{\sigma_{i, \text { stat }}^{2}+\sigma_{i, \text { uncorr }}^{2}}\left[\left(D_{i}-T_{i}(\mathbf{p})-\sum_{k=1}^{K} r_{k} \beta_{i k}\right)^{2}\right]+\sum_{k=1}^{K} r_{k}^{2}
$$

The minimisation can then be performed with respect to both the fit parameters and the systematic parameters, therefore including the effect of the systematic fluctuations to the measurement in the fit result. When multiple experiments are included in PDF fits, the number of parameters to minimise becomes large and the simultaneous minimisation of all of them might not be desirable. The equivalent approach of using a $\chi^{2}$ that includes the covariance matrix $V_{i j}$ as in Equations 7.7

$$
\begin{aligned}
\chi^{2}(\mathbf{p}) & =\sum_{i, j=1}^{N}\left(D_{i}-T_{i}(\mathbf{p})\right) V_{i j}^{-1}\left(D_{i}-T_{i}(\mathbf{p})\right) \\
V_{i j} & =\left(\sigma_{i, \text { stat }}^{2}+\sigma_{i, \text { uncorr }}^{2}\right) \delta_{i j}+\sum_{k=1}^{K} \beta_{k i} \beta_{k j}
\end{aligned}
$$


presents an equivalent practical difficulty since it involves the inversion of a large matrix that could have a singular behaviour if the correlated systematic uncertainties dominate.

A solution that is mathematically equivalent to these two methods is to minimise the $\chi^{2}$ in Equation 7.5 analytically with respect to the systematic shifts $\mathbf{r}$, as shown in Reference [164] - The actual fit minimisation then only needs to be performed with respect to the theory parameters $\mathbf{p}$, while the best values of the systematic shifts are given by the analytic minimisation. The form of the $\chi^{2}$ used in the fit is:

$$
\begin{array}{r}
\chi^{2}(\mathbf{p})=\chi^{2}(\mathbf{p}, \mathbf{r}(\mathbf{p}))=\sum_{i=1}^{N} \frac{\left(D_{i}-T_{i}(\mathbf{p})\right)^{2}}{\sigma_{i, \text { stat }}^{2}+\sigma_{i, \text { uncorr }}^{2}}-\sum_{k, l=1}^{K} B_{k} A_{k l}^{-1} B_{l} \\
B_{k}(\mathbf{p})=\sum_{i=1}^{N} \frac{\beta_{i k}\left(T_{i}(\mathbf{p})-D_{i}\right)}{\sigma_{i, \text { stat }}^{2}+\sigma_{i, \text { uncorr }}^{2}} \\
A_{k l}(\mathbf{p})=\delta_{k l}+\sum_{i=1}^{N} \frac{\beta_{i k} \beta_{i l}}{\sigma_{i, \text { stat }}^{2}+\sigma_{i, \text { uncorr }}^{2}}
\end{array}
$$

where the indices $k$ and $l$ run on the number of systematic sources $K$. The advantage of this procedure is that in a PDF fit where the errors from different datasets coming from different experiments are generally uncorrelated the matrix $A$ can be inverted in blocks for each separate experiment. Furthermore, the procedure gives an estimate of the best values of the systematic shifts using the $A$ and $B$ matrices as:

$$
r_{k}(\mathbf{a})=\sum_{l=1}^{L}\left(A_{k l}^{-1}\right) B_{l}
$$

In this treatment, systematic (nuisance) parameters are effectively allowed to vary in the fit, and the theoretical prediction will move coherently according to the correlations of each source.

Another improvement to the $\chi^{2}$ in terms of treatment of errors is the separate treatment of the statistical uncertainties that are proportional to $\sqrt{N}$ and of the systematic uncertainties that are generally multiplicative errors, as described in References [21,165]. 


\subsection{Details on PDF analysis with ATLAS and HERA data}

The baseline for the tests that include ATLAS data is the HERAPDF analysis, used to derive the set of PDF numbered with 1.5 [158]. The same DIS data as used in the HERAPDF fits is used as a starting point for the analysis in this thesis. The choice of the PDF parametrisation used for this analysis at the starting scale $Q_{0}^{2}$ is the same as the HERAPDF 1.5, with further flexibility in the parameters fitted, as reported in Section 7.3.2. There are 134 sources of systematic uncertainties for the 674 points included in the HERAPDF 1.5 analysis, but only the last three need to be treated as correlated. HERA PDF fits only use a consistent dataset (described in Section 7.3.1) with well understood systematic uncertainties, and the $\chi^{2}$ tolerance can be set to unity. The specific software used for this study is the ZEUS PDF fitting package [166]. A further note on the model and parametrisation uncertainties included in the HERAPDF 1.5 set is found in Section 7.3.3, although these are not determined for the analysis in this thesis.

\subsubsection{HERAPDF dataset}

The HERAPDF dataset includes a combination of HERA-I and HERA-II data taken at a proton beam energy of 820 and $920 \mathrm{GeV}$. The measurements included are the ZEUS and H1 combination of the neutral current and charged current for both $e^{+} p$ and $e^{-} p$ scattering (the measurement is a preliminary update of [21]). The kinematic range of the full set of data point spans $6 \cdot 10^{-7}<x<0.65$ and $0.045<Q^{2}<30000 \mathrm{GeV}^{2}$, although only data points with $Q^{2}>Q_{\text {min }}=3.5 \mathrm{GeV}^{2}$ are used so that the PDF analysis is performed in a valid region for the perturbative DGLAP evolution. The charged current measurements provide information on the high $x$ valence PDFs for both up and down flavours, while the neutral current constrains the low $x$ sea. The difference between the NC e+ and e- cross-sections at high $Q^{2}$ gives information on the valence quark PDF for $x \gtrsim 10^{-2}$. Information on the gluon PDF are obtained via scaling violations of the structure functions.

\subsubsection{Starting scale parametrisation and parameter fitted}

The PDFs parametrised in the HERAPDF analysis are chosen based on the structure functions that appear in deep inelastic scattering cross sections. These are:

- the up and down valence quark PDFs $\left(u_{v}, d_{v}\right)$,

- the total sea up-type and down-type antiquark PDFs $(\bar{U}, \bar{D}$, where the up-type includes antiup and anticharm and the down-type includes antidown, antistrange and antibottom), 
- the gluon PDF,

- the strange quark PDF, derived from the down-type antiquark PDF.

The initial PDF parametrisation used in this analysis for the quark and antiquark PDF, at the starting scale $Q_{0}^{2}=1.9 \mathrm{GeV}^{2}$, are of the form:

$$
x f(x)=A x^{B}(1-x)^{C}\left(1+D x+E x^{2}+\epsilon \sqrt{x}\right),
$$

where in the following the coefficients for the specific parton PDFs will be denoted with the parton subscript.

The $D, E$ and $\epsilon$ coefficients are introduced one by one in the fit and only kept if the $\chi^{2}$ is improved. In this analysis (and in the HERAPDF 1.5 one) the only non-zero coefficient is $E_{u_{v}}$ for the up valence quark PDF. The $B$ coefficients for both sea up- and down-types are the same, in order to keep a single coefficient for the sea. In the ZEUS fitting code, the $\bar{U}$ and $\bar{D}$ are combined in a single sea PDF that is twice their sum. The normalisation for the down-type antiquarks is defined in terms of that for the whole sea $\left(A_{\text {sea }}\right)$. The conversion between the normalisation for the down-type antiquarks and that for the sea is $A_{\bar{D}}=\left(A_{\text {sea }} / 2\right) *\left(1-f_{c}\right) /\left(2-f_{s}-f_{c}\right)$, where the fraction of charm quark at the starting scale (below the charm threshold) is $f_{c}=0$ and the fraction of strange quark is $f_{s}=0.31$ (from Reference [167]).

The DGLAP evolution of the gluon PDF is very fast: even with a flat distribution as input at $Q_{0}^{2}$, it quickly becomes very steeply rising at low $x$ for $Q^{2}>Q_{0}^{2}$. Conversely, for $Q^{2}<Q_{0}^{2}$ the gluon PDF turns over, becoming valence-like in shape or even becoming negative at low $x$ and $Q^{2}$. Since the choice of $Q_{0}^{2}$ and of the initial PDF parameterisation is arbitrary, some of the $\mathrm{PDF}$ analyses add a term to the gluon parametrisation so that it has the freedom to become negative at values below the starting scale ${ }^{4}$. This follows the behaviour observed by the ZEUS and MRST PDF analyses when evolving down the analytic forms to low $Q^{2}$. An extra term is added to Equation 7.12 for the parametrisation of the gluon density:

$$
x g(x)=A_{g} x_{g}^{B}(1-x)^{C_{g}}-\left|A_{g}^{\prime}\right| x^{B_{g}^{\prime}}(1-x)^{C_{g}^{\prime}}
$$

The coefficient $C^{\prime}$ is fixed to the value of 25 , high enough to avoid contributions from this term at large values of $x$.

\footnotetext{
${ }^{4}$ This is not necessarily worrisome because the gluon density is not an observable quantity and it has to be convoluted with coefficient functions. On the other hand if the structure function related to the gluon PDF were predicted to have the same negative behaviour, it would indicate a breakdown of the DGLAP formalism at these scales. This is also hinted at by the values of the $B$ coefficients for gluon and sea: at low $Q^{2}$, $B_{g}>B_{\text {sea }}$, contradicting the common idea that sea quarks are produced mainly from gluon splitting.
} 
The normalisation coefficients $A$ for the valence quark and gluon PDFs are constrained by the proton sum rules (the number of up and down valence quarks is fixed to 2 and 1 respectively) and the momentum sum rule.

The strange quark PDF $x s(x)$ is obtained using the estimated fraction of strange quarks in the sea $f_{s}$ as:

$$
x s(x)=f_{s} \bar{D}(x)
$$

The normalisation of the sea-type up and down quarks is also constrained using the fraction of strange quarks as $A_{\bar{u}}=A_{\bar{d}}\left(1-f_{s}\right)$, so that if $x \rightarrow 0$ anti-up and anti-down PDFs have the same behaviour. A total of 13 parameters are left free in the fits performed in this study: they are shown in Table 7.1 together with their starting value.

\begin{tabular}{|l|l|l|l|}
\hline PDF parameter & Starting value & $\begin{array}{l}\text { Value } \pm \text { uncertainty, } \\
\text { anti- } k_{t} \mathrm{R}=0.4\end{array}$ & $\begin{array}{l}\text { Value } \pm \text { uncertainty, } \\
\text { anti- } k_{t} \mathrm{R}=0.6\end{array}$ \\
\hline$B_{u_{v}}$ & 0.718 & $0.711 \pm 0.025$ & $0.707 \pm 0.024$ \\
\hline$C_{u_{v}}$ & 4.441 & $4.498 \pm 0.093$ & $4.522 \pm 0.089$ \\
\hline$E_{u_{v}}$ & 7.7 & $8.8 \pm 1.2$ & $9.1 \pm 1.2$ \\
\hline$B_{d_{v}}$ & 0.766 & $0.790 \pm 0.075$ & $0.793 \pm 0.076$ \\
\hline$C_{d_{v}}$ & 4.79 & $4.55 \pm 0.33$ & $4.53 \pm 0.31$ \\
\hline$B_{g}^{\prime}$ & -0.287 & $-0.435 \pm 0.086$ & $-0.470 \pm 0.080$ \\
\hline$A_{g}^{\prime}$ & 1.36 & $0.84 \pm 0.16$ & $0.76 \pm 0.13$ \\
\hline$A_{s e a}$ & 0.575 & $0.612 \pm 0.029$ & $0.618 \pm 0.028$ \\
\hline$B_{\bar{D}}$ & -0.1594 & $-0.1512 \pm 0.0063$ & $-0.1496 \pm 0.0061$ \\
\hline$C_{\bar{U}}$ & 3.71 & $4.02 \pm 0.54$ & $4.01 \pm 0.54$ \\
\hline$C_{\bar{D}}$ & 2.9 & $4.2 \pm 1.1$ & $4.5 \pm 1.2$ \\
\hline$B_{g}$ & -0.23 & $-0.39 \pm 0.11$ & $-0.43 \pm 0.10$ \\
\hline$C_{g}$ & 7.50 & $5.40 \pm 0.80$ & $4.96 \pm 0.70$ \\
\hline
\end{tabular}

Table 7.1.:HERAPDF 1.5 PDF parameters, starting values and fitted values after introducing the ATLAS inclusive jet measurement in the fit (anti- $k_{t} \mathrm{R}=0.4$ for the first column, anti- $k_{t}$ $\mathrm{R}=0.6$ for the second column).

The differences with respect to the publicly available parametrisation of HERAPDF 1.5 are the negative gluon term $\left(A_{g}^{\prime}, B_{g}^{\prime}\right.$ are not fixed to zero) and separate $B$ parameters for the upand down-type valence quarks. 
The chosen value of $\alpha_{s}\left(M_{z}\right)$ in this analysis and in the HERAPDF 1.5 fit is 0.1176 , as determined independently from deep inelastic scattering data [168]. The value of $\alpha_{s}\left(M_{z}\right)$ is particularly important for the gluon PDF that always enters the DGLAP evolution with factors of $\alpha_{s}$. The treatment of heavy quarks takes into account their mass using the Global Mass Variable Flavor Number Scheme (GM-VFNS $[169,170]$ ), with the charm and bottom quark masses set to $1.4 \mathrm{GeV}$ and $4.75 \mathrm{GeV}$ respectively. The starting scale $Q_{0}^{2}$ for the HERAPDF fit is $1.9 \mathrm{GeV}^{2}$, chosen to be below the charm mass threshold. The DGLAP evolution is performed using the QCDNUM [171] program for the DIS data and APPLGrid for the ATLAS jet data, using $Q^{2}$ as renormalisation and factorisation scales, in the $\bar{M} S$ renormalisation scheme [172].

\subsubsection{HERAPDF: model and parametrisation uncertainties}

HERAPDF sets also provide model and parametrisation uncertainties through varying some of the above assumptions, scales and quark masses, as described in [21]. The model uncertainties include the variation of:

- the threshold for the inclusion of DIS data $Q_{m i n}^{2}$;

- the charm and bottom masses $m_{c}, m_{b}$;

- the fraction of strange quarks $f_{s}$ at the starting scale.

The parametrisation uncertainties are estimated allowing the $D$ parameter in Equation 7.12 to be non-zero for the $u_{v}$ and gluon PDFs, and taking the envelope of the maximum deviation of the PDFs at each value of $x$. Furthermore, the starting scale $Q_{0}^{2}$ is varied to evaluate the effect of the evolution of the parametrisation starting from a different scale.

\subsection{Results: HERAPDF1.5 and ATLAS data}

The ATLAS jet data from the inclusive jet cross section was added to the HERAPDF 1.5 data, leading to a total of 764 data points included in the PDF analysis. The number of data points minus the number of parameters will be treated as the number of degrees of freedom $N_{D o F}$ in the following. Separate analyses have been performed for the two jet distance parameters.

The correlations of systematic uncertainties in the jet data detailed in Section 6.4.8 have been treated using the method that analytically minimises the $\chi^{2}$ in terms of the nuisance parameters $r_{k}$. The positive and negative uncertainties on each data point $\sigma^{+}, \sigma^{+}$for each source have been symmetrised using the following formula: 


$$
\operatorname{sign}\left(\sigma^{+}\right) *\left(\left|\sigma^{+}\right|+\left|\sigma^{-}\right|\right) / 2 .
$$

The unsigned average acquires the sign of the positive uncertainty, to take into account the cases when the positive error is a downwards one and negative error is an upwards one (to preserve anticorrelations).

For the analysis including anti- $k_{t}$ jets with distance parameter $\mathrm{R}=0.4$, the total $\chi^{2}$ is 782 , leading to a reduced $\chi^{2} / N_{D o F}$ of 1.04 . The breakdown of the $\chi^{2}$ for the two datasets is 734 for the 674 HERA data points and 48 for the 90 ATLAS data points. The total $\chi^{2}$ for anti- $k_{t}$ $\mathrm{R}=0.6$ jets is $795\left(\chi^{2} / N_{D o F}=1.06\right)$, of which 736 from HERA data and 59 from ATLAS jet data. In the treatment of the systematic uncertainties outlined in Section 7.2.1, the nuisance parameters $r_{k}$ are shifted by no more than 1.1 sigma.

\subsubsection{Impact of ATLAS jet data on PDF fits}

The results of the PDF analysis including ATLAS jet data for a number of selected PDFs at the starting value of $Q_{0}^{2}=1.9$ are shown in Figures 7.2- 7.5, while the best fit parameters for the chosen PDFs are shown in Table 7.1. The layout of the plots shows the PDFs extracted from a fit to HERA data only (in red) with the PDFs extracted from a fit to HERA+ATLAS jet data superimposed (in dark blue). The bottom panel shows the relative uncertainty in the shaded areas (in yellow shading for the HERA-only analysis and in blue lines for the HERA+ATLAS analysis and the ratio between the central PDF values (HERA/HERA+ATLAS) as the blue line.

The inclusion of the ATLAS jet data has the following effects on the parton distribution functions:

- The shape of the gluon distribution hardens from $x \approx 0.02$, and its relative uncertainty is reduced. The change is significant with respect to the uncertainties for $x>0.1$. Further tests (by excluding in turn high- $p_{T}$ jets and forward $\eta$ bins from the fit) show that this effect is mostly due to the forward pseudorapidity bins.

- The $q / \bar{q}$ sea is influenced by the changes in the gluon distribution, and shows a slightly less steep shape starting from $x=0.1$.

- Including the ATLAS jet data does not make a relevant difference for the up valence quark, since it is already well constrained by HERA data. Some improvement in the PDF 


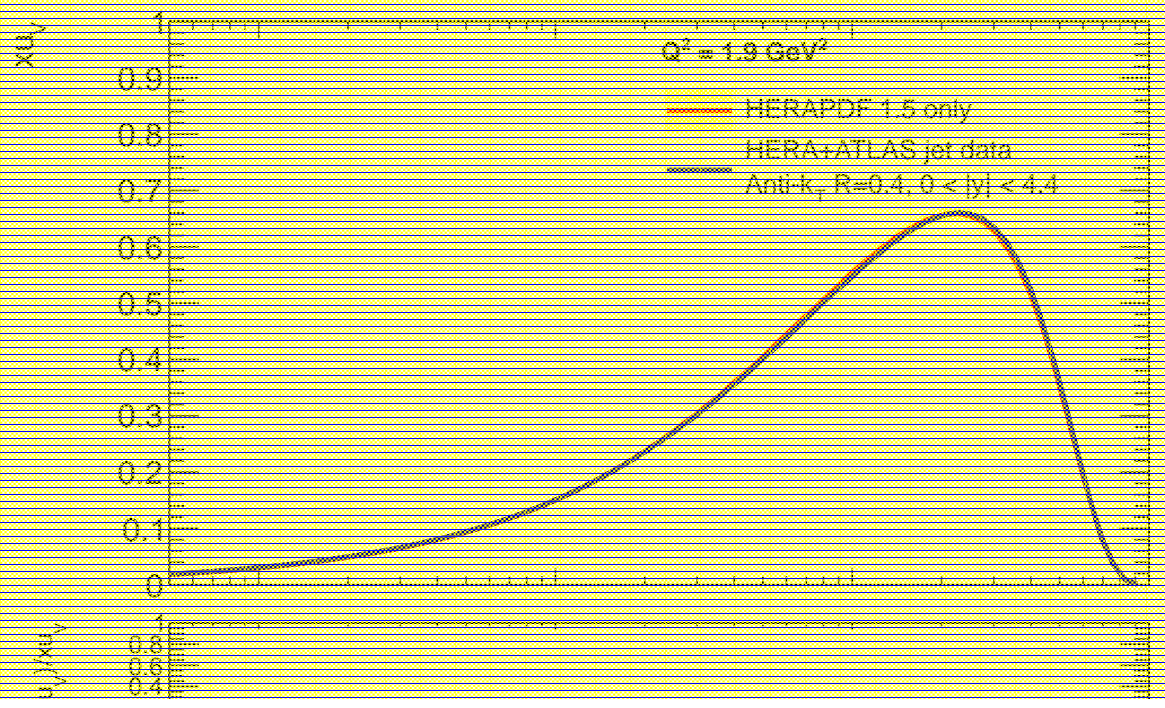

(a)

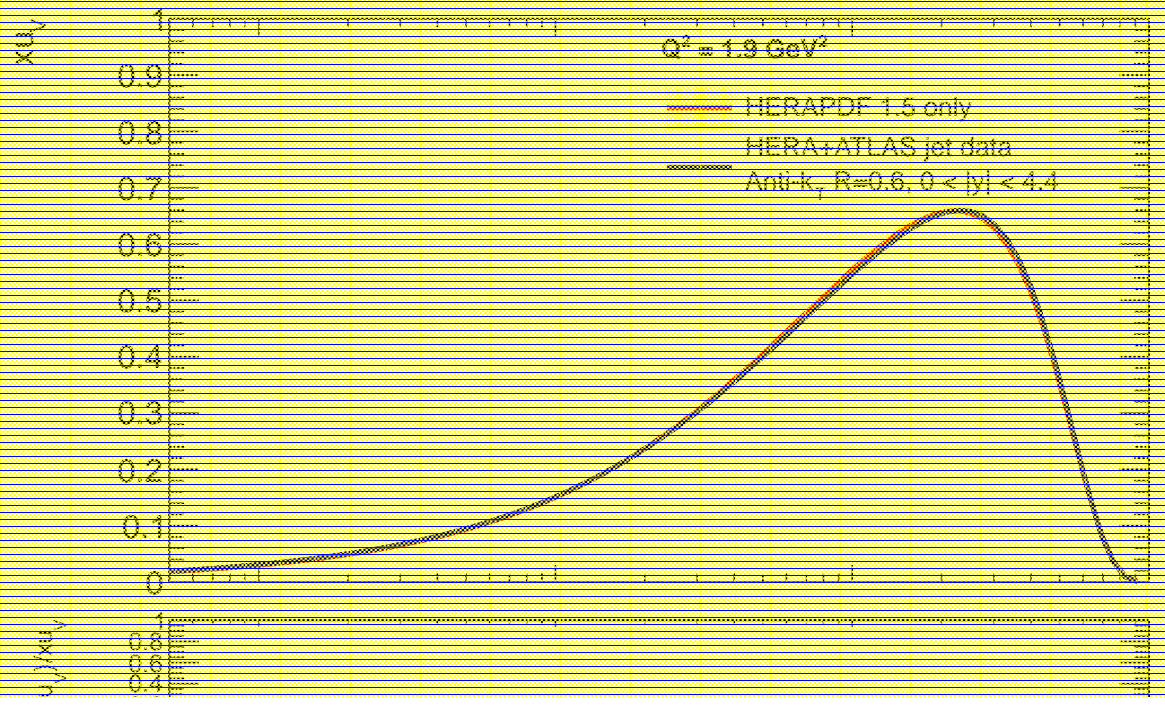

(b)

Figure 7.2.:Results of the PDF analysis including ATLAS jet data (blue with dashed uncertainties) for the up-type valence quark PDF at the starting scale of $Q_{0}^{2}=1.9 \mathrm{GeV}^{2}$. Anti- $k_{t}$ jets with distance parameter $\mathrm{R}=0.4$ are shown in Figure (a), while anti- $k_{t} \mathrm{R}=0.6$ jets are shown in Figure (b). 

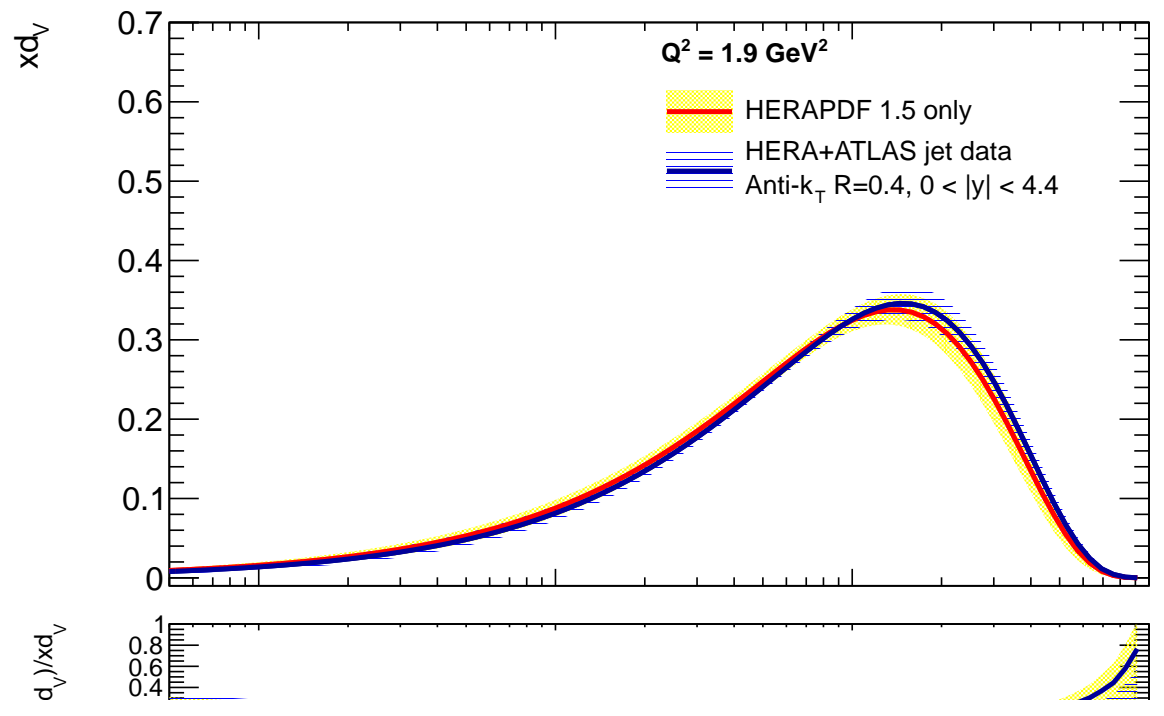

(a)

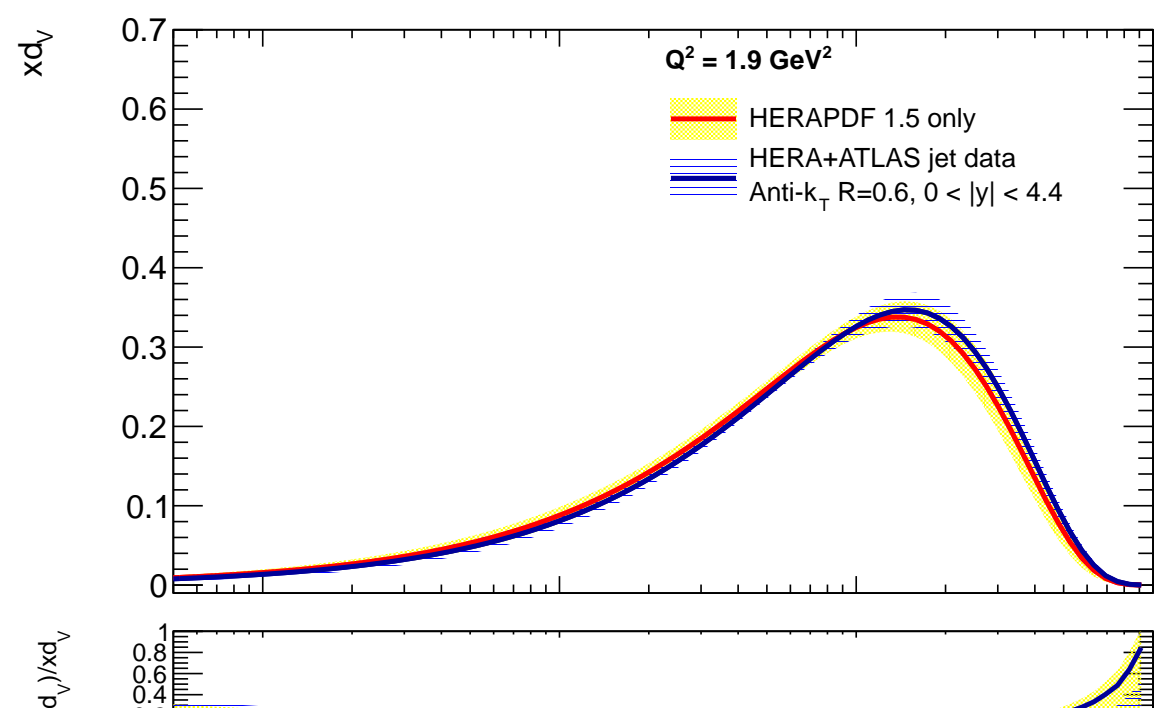

(b)

Figure 7.3.:Results of the PDF analysis including ATLAS jet data (blue with dashed uncertainties) for the down-type valence quark PDF at the starting scale of $Q_{0}^{2}=1.9 \mathrm{GeV}^{2}$. Anti- $k_{t}$ jets with distance parameter $\mathrm{R}=0.4$ are shown in Figure (a), while anti- $k_{t} \mathrm{R}=0.6$ jets are shown in Figure (b). 


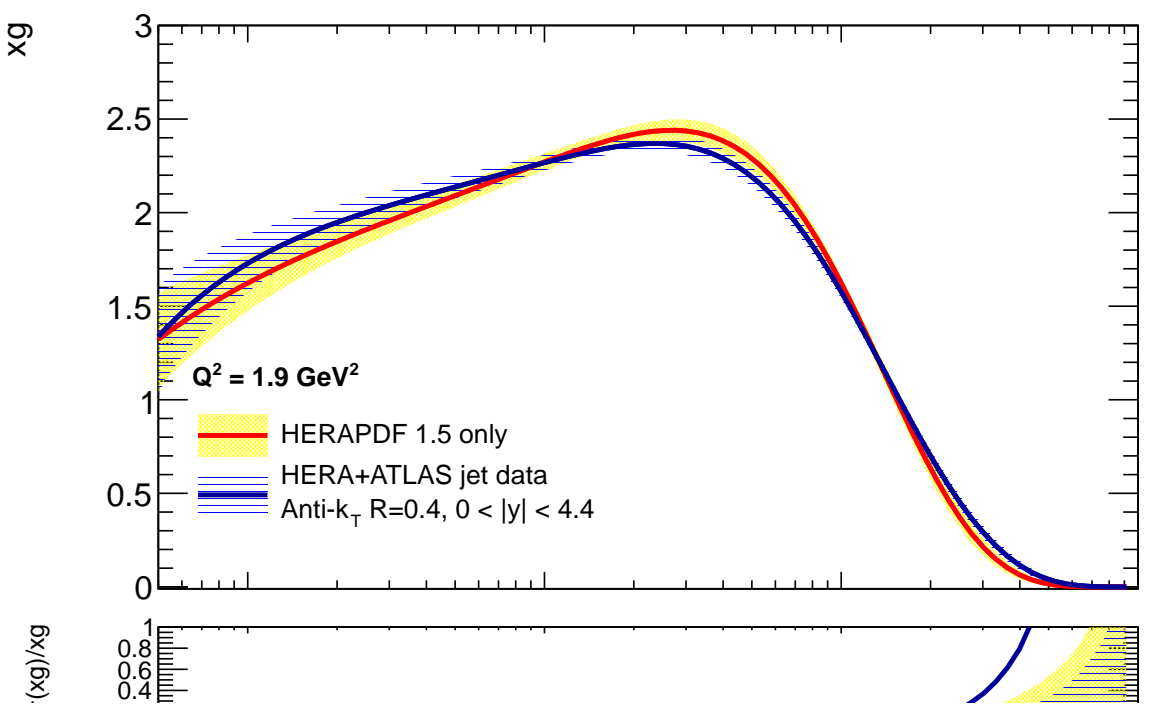

(a)

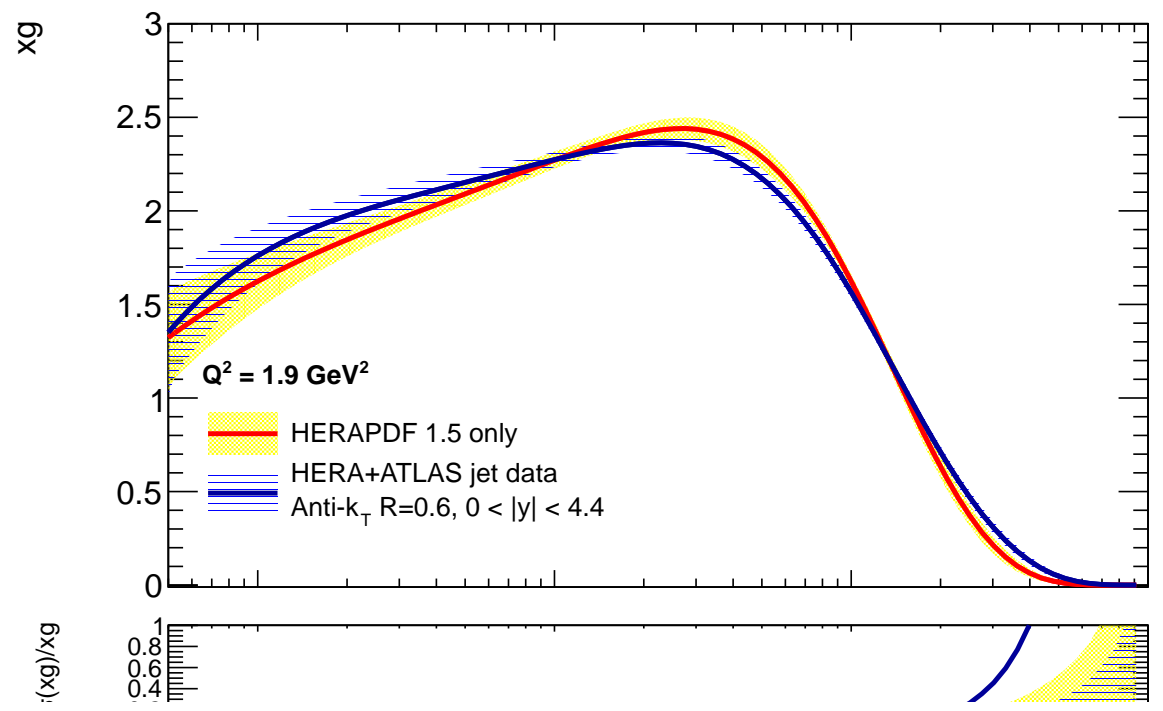

(b)

Figure 7.4.:Results of the PDF analysis including ATLAS jet data (blue with dashed uncertainties) for the gluon PDF at the starting scale of $Q_{0}^{2}=1.9 \mathrm{GeV}^{2}$. Anti- $k_{t}$ jets with distance parameter $\mathrm{R}=0.4$ are shown in Figure (a), while anti- $k_{t} \mathrm{R}=0.6$ jets are shown in Figure (b). 


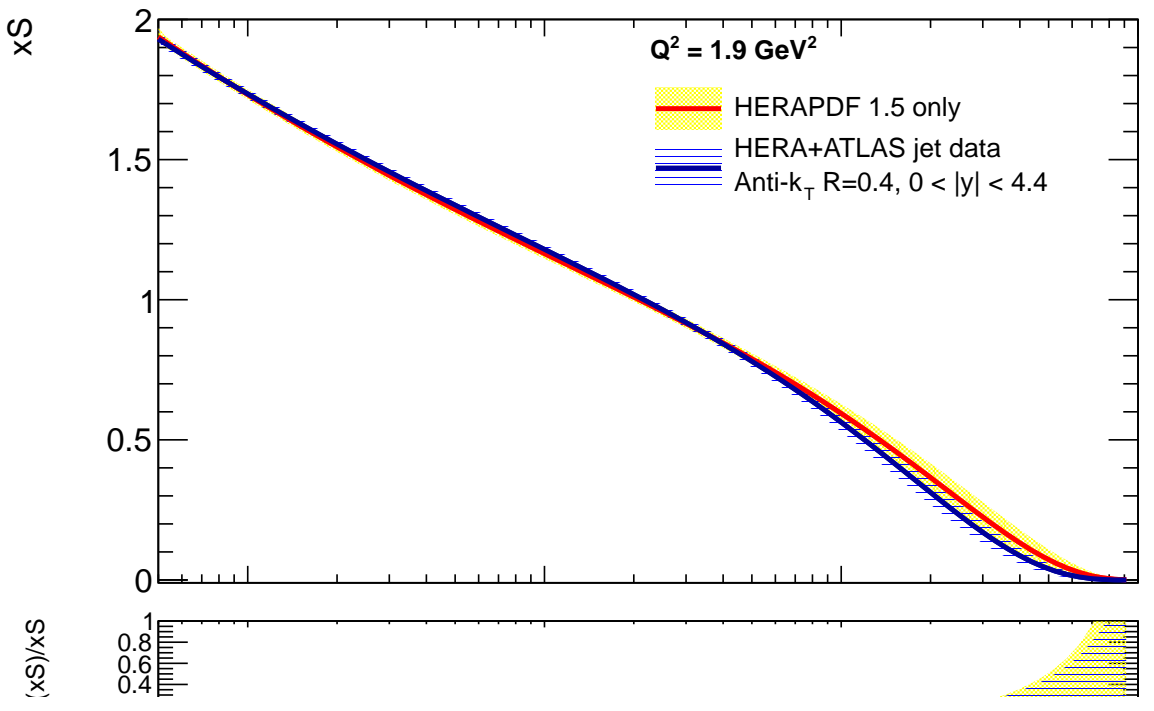

(a)

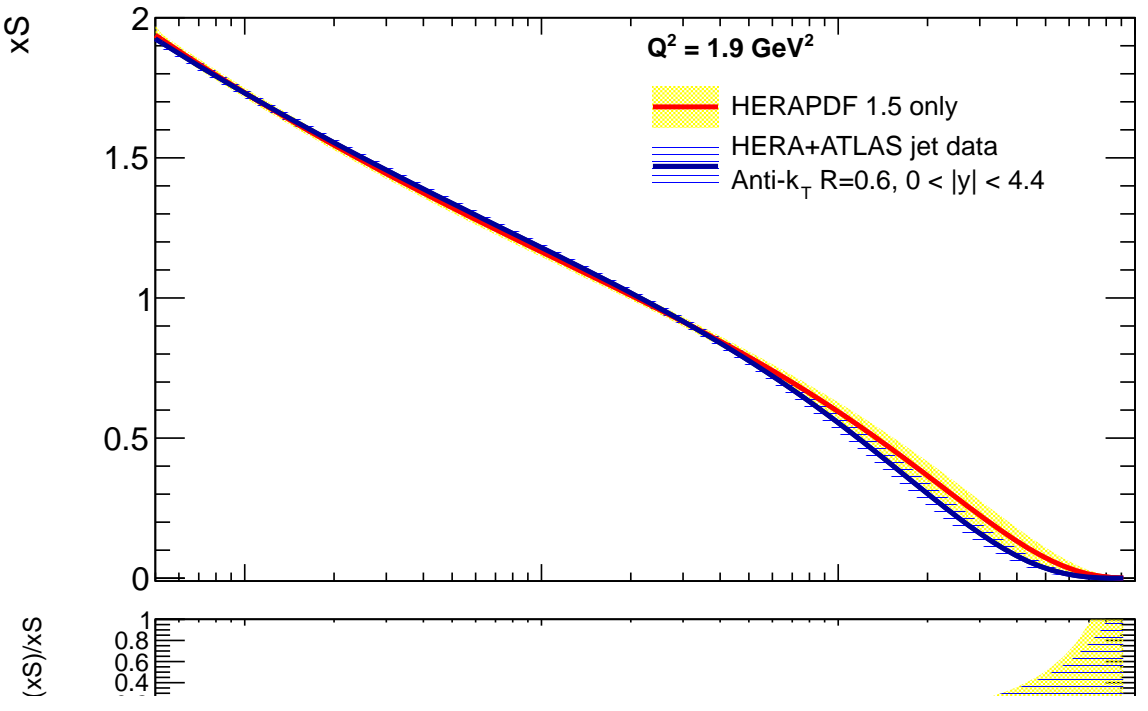

(b)

Figure 7.5.:Results of the PDF analysis including ATLAS jet data (blue with dashed uncertainties) for the sea PDF (all quark and antiquark except for the valence distributions) at the starting scale of $Q_{0}^{2}=1.9 \mathrm{GeV}^{2}$. Anti- $k_{t}$ jets with distance parameter $\mathrm{R}=0.4$ are shown in Figure (a), while anti- $k_{t} \mathrm{R}=0.6$ jets are shown in Figure (b). 
uncertainty and a slightly harder shape can be noticed for the down-type valence quark at high $x$.

The DGLAP evolution leads the gluon to rise steeply at low $x$, and the changes at high $x$ at the starting scale propagate to lower $x$, as shown in Figure 7.6.

\subsubsection{Determination of $\alpha_{s}\left(M_{Z}^{2}\right)$ with ATLAS jet data}

The strong coupling constant $\alpha_{s}$ enters the DGLAP equations, and its value at the $\mathrm{Z}$ mass can therefore be determined from a PDF fit if it is considered as a free parameter instead of fixing it to a given value as it had been done in the PDF analysis of Section 7.4.1. Furthermore, if $\alpha_{s}$ is left free the strong correlations with the gluon parameters can be accounted for in the fit.

When a fit is performed with the same starting parameters as those in Table 7.1 and $\alpha_{s}\left(M_{Z}^{2}\right)$ is left free starting from a value of 0.1176 , its value after the minimisation when the ATLAS jet data with distance parameter $\mathrm{R}=0.4$ is included in the fit is

$$
\alpha_{s}^{A T L A S ~ j e t s}\left(M_{Z}^{2}\right)=0.1209 \pm 0.0019
$$

If only HERA data is fitted, the final value of $\alpha_{s}\left(M_{Z}^{2}\right)$ does not vary as much from its starting value, and its error is larger:

$$
\alpha_{s}^{\text {No jets }}\left(M_{Z}^{2}\right)=0.1164 \pm 0.0031
$$

Even though there are no estimates of the model or parametrisation uncertainties, the values of $\alpha_{s}\left(M_{Z}^{2}\right)$ from both analyses above are compatible with the value $\alpha_{s}^{\text {GFitter }}\left(M_{Z}^{2}\right)=0.1193 \pm 0.0028$ from the global Standard Model fit by the GFitter collaboration [173,174] within the current uncertainties. The observation of a higher $\alpha_{s}$ value with respect to the default of 0.1176 when including jet data is also consistent with that noted in the HERA determination in Reference [175] $\left(\alpha_{s}^{H E R A}\right.$ jets $\left(M_{Z}^{2}\right)=0.1202 \pm 0.0013$, where only the experimental uncertainties have been mentioned).

The gluon distribution at $Q^{2}=10 \mathrm{GeV}^{2}$ that is output of the PDF fits with $\alpha_{s}\left(M_{Z}^{2}\right)$ left as a free parameter is shown in Figure 7.7: a higher value of $\alpha_{s}\left(M_{Z}^{2}\right)$ leads to a softer gluon distribution at low $x$. 


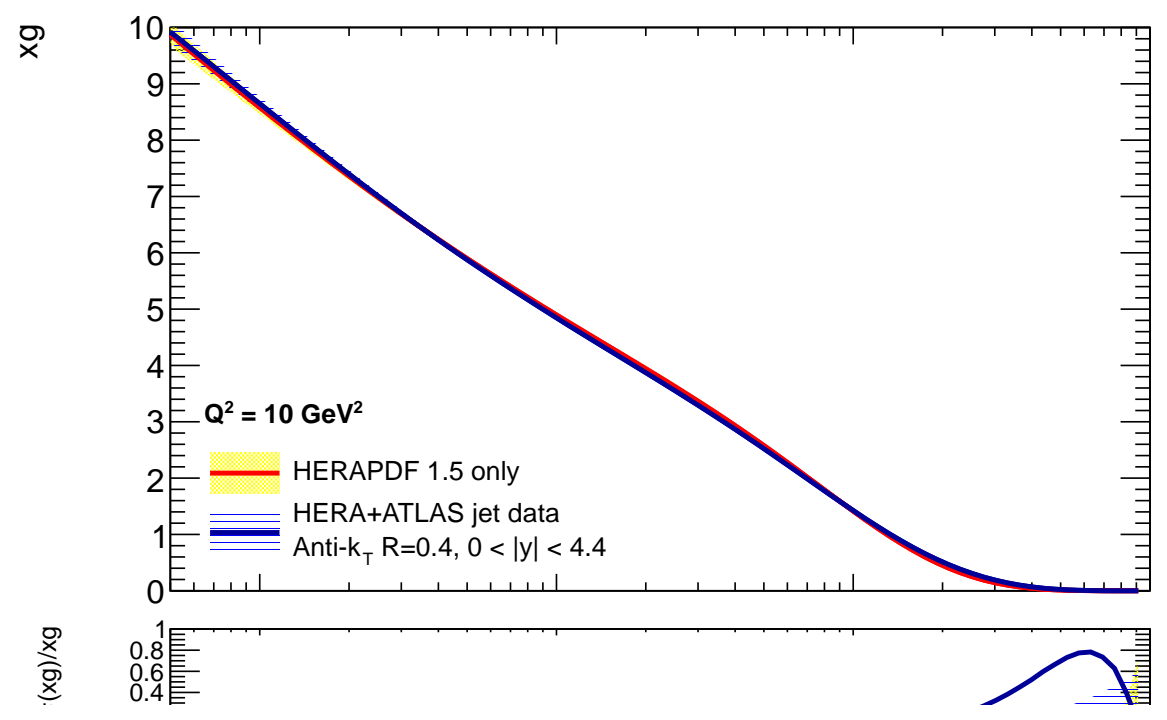

(a)

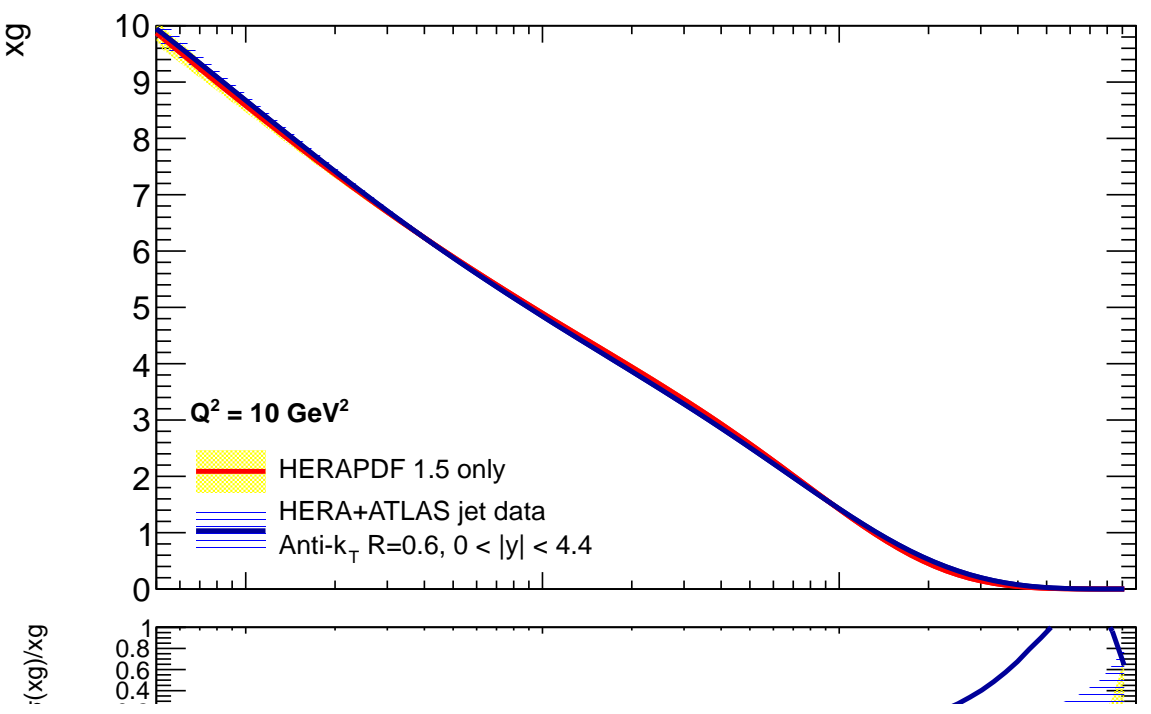

(b)

Figure 7.6.:Results of the PDF analysis including ATLAS jet data (blue with dashed uncertainties) for the gluon PDF, evolved to the scale of $Q^{2}=10 \mathrm{GeV}^{2}$. Anti- $k_{t}$ jets with distance parameter $\mathrm{R}=0.4$ are shown in Figure (a), while anti- $k_{t} \mathrm{R}=0.6$ jets are shown in Figure (b). 

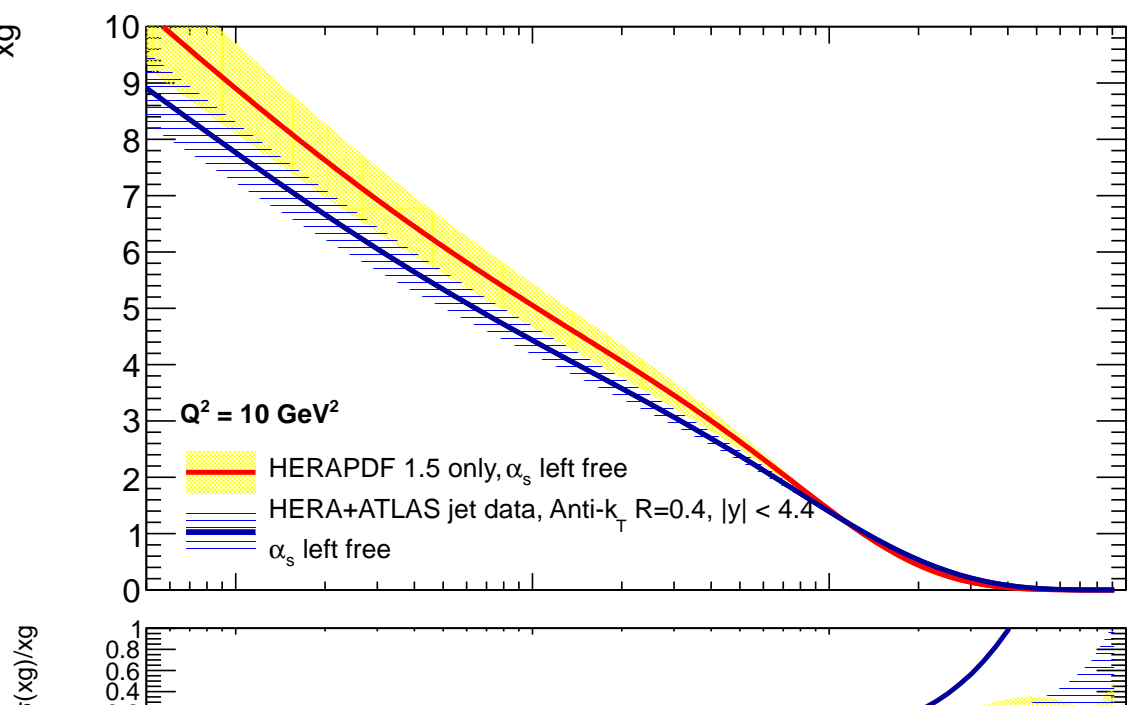

Figure 7.7.:Results of the PDF analysis including ATLAS jet data for jets with distance parameter $\mathrm{R}=0.4$ (blue with dashed uncertainties) for the gluon PDF with $\alpha_{s}\left(M_{Z}^{2}\right)$ left as a free parameter, evolved to the scale of $Q^{2}=10 \mathrm{GeV}^{2}$.

\subsubsection{Impact of reduced JES uncertainty}

A further test of the impact of ATLAS jet data on the gluon distribution can be performed assuming a rough reduction of the existing jet energy scale uncertainties. Given that the ATLAS design goal is to reduce the jet energy scale to values of approximately $1 \%$, every component of the jet energy scale is reduced to one third of its value, while the correlations are mantained. This is by no means a test with a precise physical meaning for future LHC data taking: the jet energy scale uncertainties will not be estimated using the calorimeter and Monte Carlo response as described in Chapter 5 but using in-situ techniques instead. In addition to this, the pile-up conditions are much harder in the 2011 LHC running than in the 2010 dataset, so the composition and correlations of the JES uncertainty is going to change substantially from the current estimate. Nevertheless, the results of a PDF analysis with reduced JES uncertainties can offer qualitative information on the amount of gluon density uncertainty that is driven by the uncertainty on the jet energy scale.

Only the gluon distribution is shown in Figure 7.8, where one can see that the relative uncertainty is further reduced, and the shape change becomes significant from a slightly lower value of $x$. The $\chi^{2} / N_{D o F}$ in the case of inclusion of the ATLAS jet data increases to 76 and 92 
for the 90 data points of anti- $k_{t} \mathrm{R}=0.4$ and $\mathrm{R}=0.6$ respectively, and the total $\chi^{2}$ for the whole dataset is 820 and 838 respectively, showing some tension with the HERA data.

\subsubsection{Conclusions on the PDF analysis}

The analysis performed for this thesis shows that the inclusion of the ATLAS inclusive jet cross section data in the HERAPDF analysis leads to an improvement in the knowledge of gluon distribution at high $x$. The value of the strong coupling constant at the $\mathrm{Z}$ mass peak is evaluated as a parameter of the PDF fit: the PDF analysis with the inclusion of ATLAS jet data fits it to a value of $\alpha_{s}^{A T L A S}$ jets $\left(M_{Z}^{2}\right)=0.1209 \pm 0.0019$, where the error quoted is only that on the fitted value. The uncertainty on the gluon PDF can be further reduced if the systematic uncertainty on the jet energy scale is reduced according to the ATLAS goals of $1 \%$. These results are a preliminary proof of principle that ATLAS jet data can contribute to constraining the parton distribution functions: and the theoretical/model uncertainty still need to be estimated. These results need to be integrated in an overall fit to HERA/Tevatron jets, and work is in progress to perform a combined fit together with the LHC W asymmetry data [176]. 


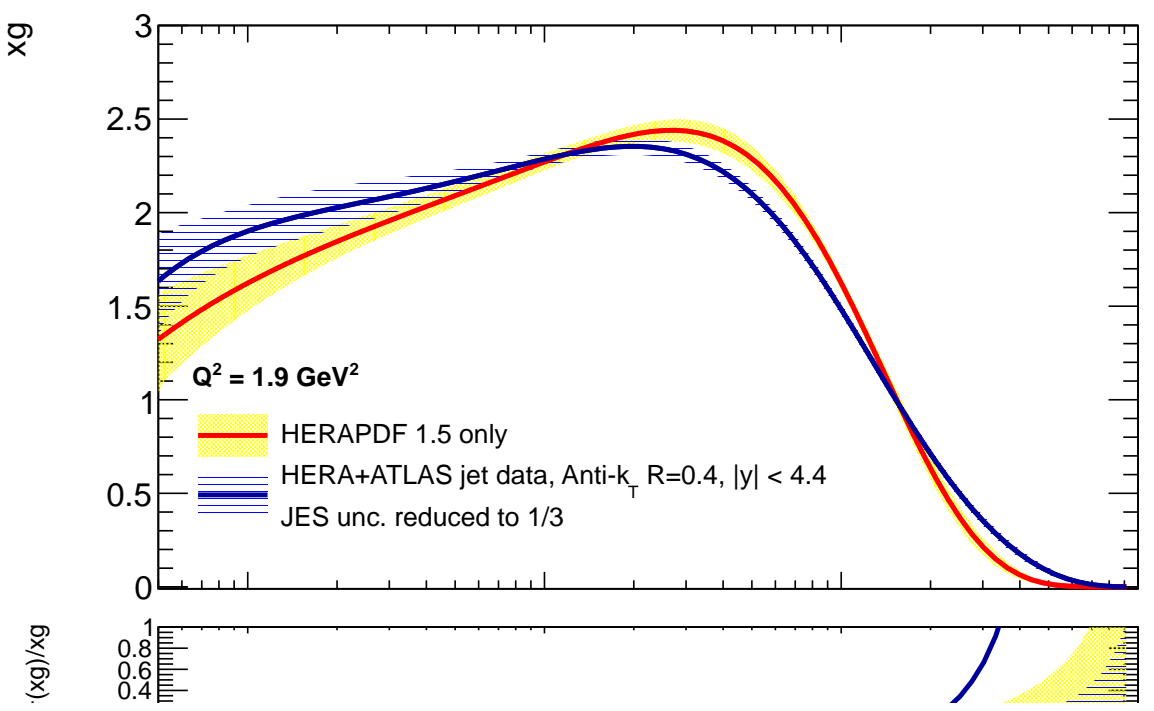

(a)

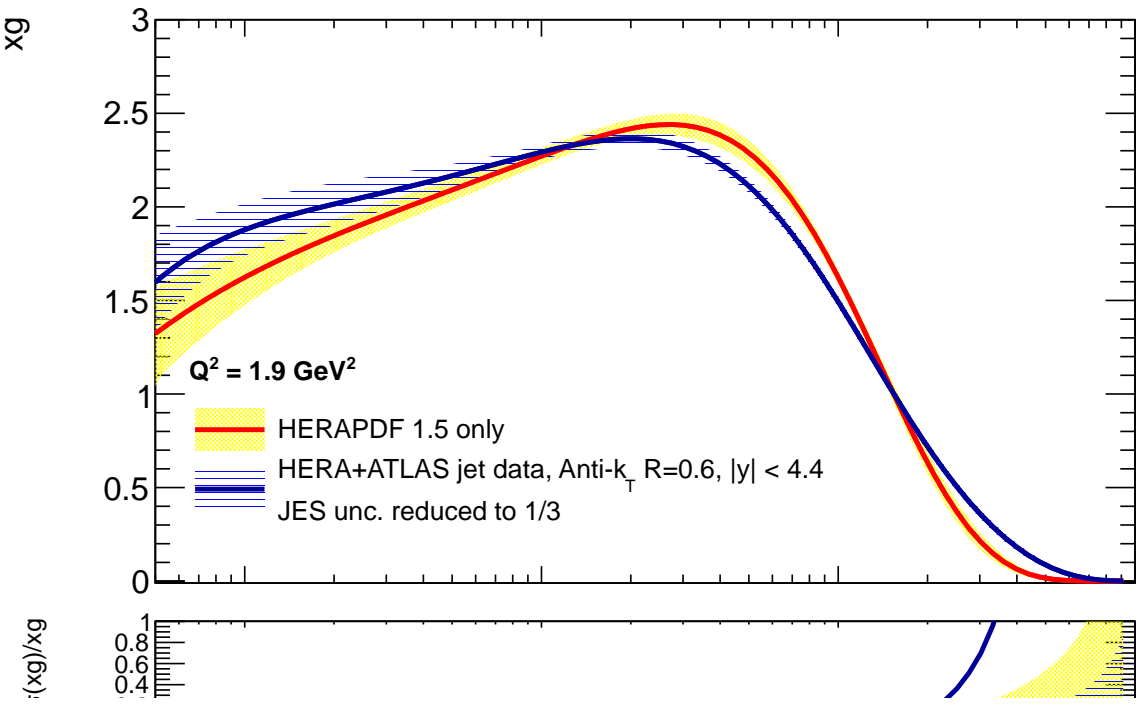

(b)

Figure 7.8.:Results of the PDF analysis including ATLAS jet data with JES uncertainty reduced to $1 / 3$ of its value (blue with dashed uncertainties) for the gluon PDF at the starting scale of $Q_{0}^{2}=1.9 \mathrm{GeV}^{2}$. Anti- $k_{t}$ jets with distance parameter $\mathrm{R}=0.4$ are shown in Figure (a), while anti- $k_{t} \mathrm{R}=0.6$ jets are shown in Figure (b). 


\section{Conclusions and outlook}

The analysis of the first ATLAS data collected from proton-proton collisions at a center-of-mass energy of $\sqrt{s}=7 \mathrm{TeV}$ at the LHC is an opportunity to test the Standard Model of particle physics to scales never reached before. The energy scale of the LHC is beyond any other particle accelerator: The theory has not yet been verified at these energies and the experiments will require tuning and calibration before being fully understood. With the beginning of data taking, processes which have previously been measured will serve as standard candles in order to test the Standard Model predictions with the new detectors. The inclusive jet cross section belongs to this category: analysis of the first data is needed to test the cutting edge technologies employed for the experiment, validate and improve jet reconstruction techniques that had previously been tested in Monte Carlo simulation and "rediscover QCD". It is also through tests of the Standard Model and QCD that we may discover new physics: significant deviations from the Standard Model predictions in unexplored regions of phase space could be a sign of of new, as yet undiscovered processes.

In order to compare data and theory, the experimental and theoretical uncertainties need to be reduced as much as possible with detailed studies of the jet reconstruction and calibration techniques and of the inputs to the theoretical predictions. It is precisely this context in which this thesis has been developed.

The experimental uncertainty on the inclusive jet cross section measurement is dominated by the systematic uncertainty on the jet energy scale: the first part of this thesis focuses on the understanding of the performance of jets using using $35 \mathrm{pb}^{-1}$ of ATLAS data collected in 2010. The JES systematic uncertainty has been evaluated for calorimeter jets reconstructed with the anti- $k_{t}$ algorithm with distance parameters $R=0.4$ and $R=0.6$, calibrated to the hadronic energy scale using the EM+JES calibration scheme, with calibrated transverse momenta $p_{\mathrm{T}}^{\text {jet }}>20 \mathrm{GeV}$ and pseudorapidities $|\eta|<4.5$. It has been estimated using a combination of in-situ techniques and an analysis of systematic variations in Monte Carlo simulations, and it is found to be of similar size for both jet distance parameters studied. The smallest JES uncertainty of less than $2.5 \%$ is found in the central calorimeter region 
$(|\eta|<0.8)$ for jets with $60 \mathrm{GeV} \leq p_{\mathrm{T}}^{\text {jet }}<800 \mathrm{GeV}$. The JES uncertainty is the largest for low- $p_{\mathrm{T}}\left(20 \mathrm{GeV} \leq p_{\mathrm{T}}^{\text {jet }}<30 \mathrm{GeV}\right)$ jets in the most forward region $3.2 \leq|\eta|<4.5$ where it amounts to $14 \%$. The additional energy due to multiple proton-proton interactions is corrected for and the remaining uncertainty in this data taking period is less than $1.5 \%$ per additional interaction for jets with $p_{\mathrm{T}}^{\text {jet }}>50 \mathrm{GeV}$ and decreases with $p_{\mathrm{T}}$. The JES is validated up to $p_{\mathrm{T}}^{\text {jet }}=1 \mathrm{TeV}$ using several in-situ methods.

The measurement of the inclusive jet cross section is reviewed and compared to theory for both jet distance parameters of 0.4 and 0.6. The kinematic range of this measurement spans more than $1 \mathrm{TeV}$ in the central bins, and the jet cross sections falls by more than seven orders of magnitude in this range. The data and theoretical prediction are in agreement within uncertainties for the whole kinematic range.

The ATLAS inclusive jet cross section measurement has been used as input to the determination of the parton distribution functions using the ZEUS PDF fitting code in a proof of principle analysis to show that ATLAS data can have a non-negligible impact on PDF fits, even when the dominant JES uncertainty has not yet reached the desired $1 \%$ precision. The inclusion of ATLAS jet data together with data from the HERA collider as a baseline leads to a harder gluon PDF with reduced uncertainties. It also allows to estimate the strong coupling constant at the $\mathrm{Z}$ mass peak as a parameter of the PDF fit, leading to a value of $\alpha_{s}^{A T L A S}$ jets $\left(M_{Z}^{2}\right)=$ $0.1209 \pm 0.0019$, where the error quoted is only that on the fitted value. The conclusions from this preliminary study need completion in the estimate of the theoretical, model and parameterisation uncertainties, but they can serve as a starting point for the inclusion of ATLAS jet data in a full global fit that also includes HERA and Tevatron jet data, and where the recent Tevatron, ATLAS and CMS electroweak data on the W asymmetry is included as well to constrain the slope of the $d / u$ PDFs at low $x$.

The measurement of the inclusive jet cross section in ATLAS can be further exploited in PDF fits, given that a large number of ingredients have been readied for the analysis of the full 2010 dataset. A detailed analysis of correlated uncertainties between the two jet distance parameters would allow cancellation of a number of components related to the jet energy scale uncertainties when the ratio between jet cross sections with different radii is taken. Given that this quantity can be calculated analytically to next to leading order $[177,178]$, it could be a powerful input for PDF fits. Furthermore, the jet cross section ratio could provide useful information on non perturbative effects in jets. Ratios could also be taken for jet cross sections at different LHC centre of mass energy, profiting from the $2.76 \mathrm{TeV}$ run of the beginning of 2011 [179]. 


\section{Bibliography}

[1] G. Altarelli, The Standard model of particle physics, arXiv:0510281 [hep-ph]. To appear in the 'Encyclopedia of Mathematical Physics, Elsevier'.

[2] C. Quigg, Unanswered Questions in the Electroweak Theory, arXiv:0905.3187 [hep-ph]. arXiv:0905.3187v1 [hep-ph].

[3] P. Higgs, Broken symmetries, massless particles and gauge fields, Phys. Lett. 12 (1964) no. $2,132-133$.

[4] F. Englert and R. Brout, Broken Symmetry and the Mass of Gauge Vector Mesons, Phys. Rev. Lett. 13 (1964) 321-323. http://link.aps.org/doi/10.1103/PhysRevLett.13.321.

[5] Wikipedia (adapted by C. Fitzpatrick), Standard Model - Wikipedia, The Free Encyclopedia, Online; accessed 6-june-2009, 2009.

http://en.wikipedia.org/w/index.php?title=Standard_Model.

[6] H. Yukawa, On the Interaction of Elementary Particles. I*, Progress of Theoretical Physics Supplement 1 (1955) 1-10. http://ptp.ipap.jp/link?PTPS/1/1/.

[7] C. N. Yang and R. L. Mills, Conservation of Isotopic Spin and Isotopic Gauge Invariance, Phys. Rev. 96 (1954) no. 1, 191-195.

[8] M. Gell-Mann and Y. Ne'eman, The eightfold way: with new contributions from the authors. Advanced book classics. Perseus Pub., 2000.

[9] R. Feynman, Photon-hadron interactions. Advanced book classics. Addison-Wesley, 1998.

[10] R. Cahn and G. Goldhaber, The experimental foundations of particle physics. Cambridge University Press, 2009.

[11] D. J. Gross, The discovery of asymptotic freedom and the emergence of $Q C D$, Proceedings of the National Academy of Sciences of the United States of America 102 (2005) no. 26. 9099-9108, http://www.pnas.org/content/102/26/9099.full.pdf+html.

[12] D. J. Gross and F. Wilczek, Asymptotically Free Gauge Theories. I, Phys. Rev. D 8 (1973) no. 10, 3633-3652.

[13] D. J. Gross and F. Wilczek, Asymptotically free gauge theories. II, Phys. Rev. D 9 (1974) no. 4, 980-993.

[14] S. Bethke, The 2009 World Average of $\alpha_{s}\left(M_{Z}\right)$, Eur. Phys. J. C64 (2009) 689-703, arXiv:0908.1135 [hep-ph].

[15] T. Sjostrand, Monte Carlo Generators, arXiv:0611247 [hep-ph]. 
[16] J. M. Campbell, J. W. Huston, and W. J. Stirling, Hard Interactions of Quarks and Gluons: A Primer for LHC Physics, Rept. Prog. Phys. 70 (2007) 89, arXiv:0611148 [hep-ph].

[17] J. C. Collins, D. E. Soper, and G. F. Sterman, Factorization of Hard Processes in QCD, Adv.Ser.Direct.High Energy Phys. 5 (1988) 1-91, arXiv:0409313 [hep-ph].

[18] R. K. Ellis, H. Georgi, M. Machacek, H. D. Politzer, and G. G. Ross, Perturbation theory and the parton model in QCD, Nuclear Physics B 152 (1979) no. 2, 285 - 329.

[19] R. Devenish and A. Cooper-Sarkar, Deep inelastic scattering. Oxford University Press, 2004. http://books . google.com/books?id=L68zn24GgkcC.

[20] P. Soeding, B. H. Wiik, G. Wolf, and S. L. Wu, The first evidence for three-jet events in $e^{+} e^{-}$collisions at PETRA: first direct observation of the Gluon, .

[21] H1 and ZEUS Collaboration, F. D. Aaron et al., Combined Measurement and QCD Analysis of the Inclusive ep Scattering Cross Sections at HERA, JHEP 01 (2010) 109, arXiv:0911.0884 [hep-ex].

[22] G. Altarelli and G. Parisi, Asymptotic freedom in parton language, Nuclear Physics B 126 (1977) no. 2, $298-318$.

[23] S. J. Brodsky and G. R. Farrar, Scaling laws for large-momentum-transfer processes, Phys. Rev. D 11 (1975) no. 5, 1309-1330.

[24] F. J. Gilman, Photoproduction and electroproduction, Phys. Rep. C 4 (1972) 96-151.

[25] J. C. Collins and D. E. Soper, The Theorems of Perturbative QCD, Annual Review of Nuclear and Particle Science 37 (1987) no. 1, 383-409.

[26] T. D. Lee and M. Nauenberg, Degenerate Systems and Mass Singularities, Phys. Rev. 133 (1964) B1549-B1562.

[27] T. Kinoshita, Mass singularities of Feynman amplitudes, J. Math. Phys. 3 (1962) 650-677.

[28] S. D. Drell, D. J. Levy, and T.-M. Yan, Theory of Deep-Inelastic Lepton-Nucleon Scattering and Lepton-Pair Annihilation Processes. III. Deep-Inelastic Electron-Positron Annihilation, Phys. Rev. D 1 (1970) no. 6, 1617-1639.

[29] G. Sterman and S. Weinberg, Jets from Quantum Chromodynamics, Phys. Rev. Lett. 39 (1977) no. 23, 1436-1439.

[30] CDF Collaboration, T. Aaltonen et al., Measurement of the Inclusive Jet Cross Section at the Fermilab Tevatron p-pbar Collider Using a Cone-Based Jet Algorithm, Phys. Rev. D78 (2008) 052006, arXiv:0807.2204 [hep-ex].

[31] D0 Collaboration Collaboration, V. Abazov et al., Measurement of the inclusive jet cross-section in $p \bar{p}$ collisions at $s^{1 / 2}=1.96$-TeV, Phys.Rev.Lett. 101 (2008) 062001, arXiv:0802.2400 [hep-ex].

[32] Z. Nagy, Next-to-leading order calculation of three jet observables in hadron hadron collision, Phys. Rev. D68 (2003) 094002, arXiv:0307268 [hep-ph]. 
[33] T. Carli et al., A posteriori inclusion of parton density functions in NLO QCD final-state calculations at hadron colliders: The APPLGRID Project, Eur. Phys. J. C66 (2010) 503-524, arXiv:0911.2985 [hep-ph].

[34] T. Sjostrand, S. Mrenna, and P. Z. Skands, PYTHIA 6.4 Physics and Manual, JHEP 05 (2006) 026, arXiv:0603175 [hep-ph].

[35] M. L. Mangano, M. Moretti, F. Piccinini, R. Pittau, and A. D. Polosa, ALPGEN, a generator for hard multiparton processes in hadronic collisions, JHEP 07 (2003) 001, arXiv:0206293 [hep-ph].

[36] T. Sjostrand and P. Z. Skands, Transverse-momentum-ordered showers and interleaved multiple interactions, Eur.Phys.J. C39 (2005) 129-154, arXiv:0408302 [hep-ph].

[37] B. Andersson, G. Gustafson, G. Ingelman, and T. Sjöstrand, Parton fragmentation and string dynamics, Phys. Rep. 97 (1983) 31-145.

[38] A. Sherstnev and R. S. Thorne, Parton distributions for LO generators, Eur. Phys. J. C 55 (2008) 553-575, arXiv:0711.2473 [hep-ph].

[39] G. Corcella et al., HERWIG 6.5 release note, arXiv:0210213 [hep-ph].

[40] M. L. Mangano, M. Moretti, and R. Pittau, Multijet matrix elements and shower evolution in hadronic collisions: $W b \bar{b}+n$ jets as a case study, Nucl. Phys. B 632 (2002) 343-362, arXiv:0108069 [hep-ph].

[41] B. R. Webber, A QCD model for jet fragmentation including soft gluon interference, Nuclear Physics B 238 (1984) no. 3, 492 - 528.

[42] J. M. Butterworth, J. R. Forshaw, and M. H. Seymour, Multiparton interactions in photoproduction at HERA, Z. Phys. C 72 (1996) 637-646, arXiv:9601371 [hep-ph].

[43] J. Pumplin et al., New generation of parton distributions with uncertainties from global QCD analysis, JHEP 07 (2002) 012, arXiv:0201195 [hep-ph].

[44] M. Bahr, S. Gieseke, M. Gigg, D. Grellscheid, K. Hamilton, et al., Herwig++ Physics and Manual, Eur.Phys.J. C58 (2008) 639-707, arXiv:0803.0883 [hep-ph].

[45] M. Bahr, S. Gieseke, and M. H. Seymour, Simulation of multiple partonic interactions in Herwig++, JHEP 0807 (2008) 076, arXiv:0803.3633 [hep-ph].

[46] ATLAS Collaboration, Charged particle multiplicities in pp interactions at $\sqrt{s}=0.9$ and $7 \mathrm{TeV}$ in a diffractive limited phase-space measured with the ATLAS detector at the LHC and new PYTHIA6 tune, ATLAS-CONF-2010-031, CERN, Geneva, May, 2010.

[47] ATLAS Collaboration, First tuning of HERWIG/JIMMY to ATLAS data, ATL-PHYS-PUB-2010-014, CERN, Geneva, October, 2010.

[48] ATLAS Collaboration, Response of the ATLAS calorimeter to single isolated hadrons produced in proton proton collisions a centre-of-mass energy of $\sqrt{s}=900 \mathrm{GeV}$, ATLAS-CONF-2010-017, CERN, Geneva, March, 2010.

[49] ATLAS Collaboration, ATLAS calorimeter response to single isolated hadrons and estimation of the calorimeter jet scale uncertainty, ATLAS-CONF-2010-052, CERN, Geneva, June, 2010. 
[50] ATLAS Collaboration, Inputs to jet reconstruction and calibration with the ATLAS detector using proton-proton collisions at $\sqrt{s}=900 \mathrm{GeV}$, ATLAS-CONF-2010-016, CERN, Geneva, April, 2010.

[51] ATLAS Collaboration, Properties and internal structure of jets produced in proton-proton collisions at $\sqrt{s}=900 \mathrm{GeV}$, ATLAS-CONF-2010-018, CERN, Geneva, April, 2010.

[52] ATLAS Collaboration, Observation of energetic jets in pp collisions at $\sqrt{s}=7$ TeV using the ATLAS experiment at the LHC, ATLAS-CONF-2010-043, CERN, Geneva, April, 2010 .

[53] ATLAS Collaboration, Measurement of inclusive jet and dijet cross sections in proton-proton collisions at $7 \mathrm{TeV}$ centre-of-mass energy with the ATLAS detector, Eur. Phys. J. C 71 (2011) 1512, arXiv:1009.5908 [hep-ex].

[54] ATLAS Collaboration, Properties of jets and inputs to jet reconstruction and calibration with the ATLAS detector using proton-proton collisions at $\sqrt{s}=7 \mathrm{TeV}$, ATLAS-CONF-2010-053, CERN, Geneva, June, 2010.

[55] ATLAS Collaboration, In-situ pseudo-rapidity inter-calibration to evaluate jet energy scale uncertainty and calorimeter performance in the forward region, ATLAS-CONF-2010-055, CERN, Geneva, June, 2010.

[56] ATLAS Experiment(C2011 CERN. http://www.atlas.ch/photos/index.html.

[57] ATLAS Collaboration, In-situ jet energy scale and jet shape corrections for multiple interactions in the first ATLAS data at the LHC, ATLAS-CONF-2011-030, CERN, Geneva, February, 2011.

[58] ATLAS Collaboration, G. Aad et al., Luminosity Determination in pp Collisions at sqrt(s)=7 TeV Using the ATLAS Detector at the LHC, Eur. Phys. J. C71 (2011) 1630, arXiv:1101.2185 [hep-ex].

[59] Updated Luminosity Determination in pp Collisions at root(s)=7 TeV using the ATLAS Detector, ATLAS-CONF-2011-011, CERN, Geneva, Mar, 2011.

[60] ATLAS Collaboration, The ATLAS Experiment at the CERN Large Hadron Collider, JINST 3 (2008) S08003.

[61] ATLAS Collaboration, Expected Performance of the ATLAS Experiment Detector, Trigger, Physics, CERN-OPEN-2008-020, CERN, Geneva, Sep, 2010.

[62] T. Cornelissen, M. Elsing, S. Fleischmann, W. Liebig, E. Moyse, and A. Salzburger, Concepts, Design and Implementation of the ATLAS New Tracking (NEWT), ATL-SOFT-PUB-2007-007, CERN, Geneva, Mar, 2007.

[63] R. Wigmans, Advances in hadron calorimetry, CERN-PPE-91-39, Feb, 1991.

[64] ATLAS Electromagnetic Liquid Argon Calorimeter Group Collaboration, B. Aubert et al., Performance of the ATLAS electromagnetic calorimeter barrel module 0, Nucl. Instrum. Meth. A500 (2003) 202-231.

[65] M. Aharrouche et al., Energy Linearity and Resolution of the ATLAS Electromagnetic Barrel Calorimeter in an Electron Test-beam, Nucl. Instrum. Meth. A 568 (2006) 601-623. 
[66] A. Bazan, The ATLAS liquid argon calorimeter read-out system, IEEE Trans. Nucl. Sci. 53 (2006) 735-740.

[67] ATLAS Collaboration. https://twiki.cern.ch/twiki/bin/view/AtlasPublic/LArCaloPublicResultsDetStatus.

[68] ATLAS Collaboration. https://twiki.cern.ch/twiki/bin/view/AtlasPublic/TileCaloPublicResults.

[69] W. Lampl et al., Calorimeter clustering algorithms: description and performance, ATL-LARG-PUB-2008-002, CERN, Geneva, April, 2008.

[70] R. Sacco, Position resolution of an ATLAS electromagnetic calorimeter module, ATL-LARG-2003-008, CERN, Geneva, Jul, 2003.

[71] J. Colas et al., Response Uniformity of the ATLAS Liquid Argon Electromagnetic Calorimeter, Nucl. Instrum. Meth. A 582 (2007) 429-455, arXiv:0709.1094 [physics.ins-det].

[72] M. Aharrouche et al., Measurement of the response of the ATLAS liquid argon barrel calorimeter to electrons at the 2004 combined test- beam, Nucl. Instrum. Meth. A 614 (2010) 400-432.

[73] E. Abat et al., Combined performance studies for electrons at the 2004 ATLAS combined test-beam, JINST 5 (2010) P11006.

[74] J. Pinfold et al., Performance of the ATLAS liquid argon endcap calorimeter in the pseudorapidity region $2.5<|\eta|<4.0$ in beam tests, Nucl. Instrum. Meth. A 593 (2008) 324-342.

[75] C. Cojocaru et al., Hadronic calibration of the ATLAS liquid argon end-cap calorimeter in the pseudorapidity region $1.6<[\eta]<1.8$ in beam tests, Nucl. Instrum. Meth. A 531 (2004) no. 3, $481-514$.

[76] M. Aharrouche et al., Study of the response of ATLAS electromagnetic liquid argon calorimeters to muons, Nucl. Instrum. Meth. A 606 (2009) 419-431.

[77] ATLAS Collaboration, Readiness of the ATLAS Liquid Argon Calorimeter for LHC Collisions, Eur. Phys. J. C 70 (2010) 1193-1236, arXiv:1007.5423 [physics.ins-det].

[78] ATLAS Collaboration, Electron performance measurements with the ATLAS detector using the 2010 LHC proton-proton collision data, ATL-COM-PHYS-2011-263 [Internal note], CERN, Geneva, In preparation, 2011.

[79] G. Usai, Signal Reconstruction of the ATLAS Hadronic Tile Calorimeter: implementation and performance, ATL-TILECAL-PROC-2010-008, CERN, Geneva, Aug, 2010.

[80] P. Adragna et al., Testbeam studies of production modules of the ATLAS Tile calorimeter, Nucl. Instrum. Meth. A 606 (2009) 362-394.

[81] ATLAS Collaboration, Readiness of the ATLAS Tile Calorimeter for LHC collisions, Eur. Phys. J. C 70 (2010) 1193-1236, arXiv:1007.5423 [physics.ins-det].

[82] Z. Weng, Calibration of the ATLAS hadronic barrel calorimeter TileCal using 2008, 2009 
and 2010 cosmic rays data, ATL-TILECAL-SLIDE-2011-247, Jun, 2011.

[83] A. Collaboration, Calibration of the ATLAS hadronic barrel calorimeter TileCal using 2008, 2009 and 2010 cosmic rays data, ATL-TILECAL-PUB-2011-001, CERN, Geneva, Sep, 2011.

[84] C. Santoni. Private communication.

[85] GEANT4 Collaboration, S. Agostinelli et al., GEANT4: A simulation toolkit, Nucl. Instrum. Meth. A 506 (2003) 250-303.

[86] ATLAS Collaboration, The ATLAS Simulation Infrastructure, Eur. Phys. J. C 70 (2010) 823-874.

[87] A. Ribon et al., Status of Geant4 hadronic physics for the simulation of LHC experiments at the LHC physics program, CERN-LCGAPP-2010-02, CERN, Geneva, May, 2010.

[88] G. Folger and J. P. Wellisch, String parton models in Geant4, arXiv:nucl-th/0306007.

[89] H. W. Bertini, Intranuclear-cascade calculation of the secondary nucleon spectra from nucleon-nucleus interactions in the energy range 340 to 2900 mev and comparisons with experiment, Phys. Rev. A 188 (1969) 1711-1730.

[90] P. Adragna et al., Measurement of pion and proton response and longitudinal shower profiles up to 20 nuclear interaction lengths with the ATLAS Tile calorimeter, Nucl. Instrum. Meth. A 615 (2010) 158-181.

[91] ATLAS Collaboration, Response and Shower Topology of 2 to $180 \mathrm{GeV}$ Pions Measured with the ATLAS Barrel Calorimeter at the CERN Test-beam and Comparison to Monte Carlo Simulations, ATL-CAL-PUB-2010-001, CERN, Geneva, May, 2010.

[92] ATLAS Collaboration, Study of energy response and resolution of the ATLAS barrel calorimeter to hadrons of energies from 20-GeV to 350-GeV, Nucl. Instrum. Meth. A 621 (2010) 134-150.

[93] ATLAS Collaboration, Study of the response of the ATLAS central calorimeter to pions of energies from 3 to $9 \mathrm{GeV}$, Nucl. Instrum. Meth. A 607 (2009) no. 2, 372 - 386.

[94] A. E. Kiryunin, H. Oberlack, D. Salihagic, P. Schacht, and P. Strizenec, GEANT4 physics evaluation with testbeam data of the ATLAS hadronic end-cap calorimeter, Nucl. Instrum. Meth. A 560 (2006) 278-290.

[95] ATLAS Collaboration, Response of isolated particles identified using resonances in proton-proton collisions at $\sqrt{s}=7 \mathrm{TeV}$ with the ATLAS detector, ATLAS-CONF-2011-019, CERN, Geneva, February, 2011.

[96] ATLAS Collaboration, Mapping the material in the ATLAS Inner Detector using secondary hadronic interactions in 7 TeV collisions, ATLAS-CONF-2010-058, CERN, Geneva, Jul, 2010.

[97] ATLAS Collaboration, Study of the Material Budget in the ATLAS Inner Detector with $K_{S}^{0}$ decays in collision data at $\sqrt{s}=900 \mathrm{GeV}$, ATLAS-CONF-2010-019, CERN, Geneva, Jul, 2010.

[98] G. Barrand et al., GAUDI - A software architecture and framework for building HEP 
data processing applications, Comput. Phys. Commun. 140 (2001) 45-55.

[99] Performance of the Minimum Bias Trigger in p-p Collisions at $\sqrt{s}=900 \mathrm{GeV}$, ATLAS-CONF-2010-025, CERN, Geneva, Jul, 2010.

[100] R. E. Kwee, Minimum Bias Trigger in ATLAS, ATL-DAQ-PROC-2010-055, CERN, Geneva, Dec, 2010.

[101] Performance of the ATLAS Jet Trigger in the Early $\sqrt{s}=7$ TeV Data, ATLAS-CONF-2010-094, CERN, Geneva, Oct, 2010.

[102] C. Buttar et al., Standard Model Handles and Candles Working Group: Tools and Jets Summary Report, arXiv:0803.0678 [hep-ph].

[103] M. Cacciari and G. P. Salam, Dispelling the $N^{3}$ myth for the $k_{t}$ jet-finder, Phys. Lett. B 641 (2006) no. 1, $57-61$.

[104] M. Cacciari, G. P. Salam, and G. Soyez. http://fastjet.fr/.

[105] M. Cacciari and G. P. Salam, Pileup subtraction using jet areas, Phys. Lett. B 659 (2008) no. $1-2,119-126$.

[106] L. Asquith et al., Performance of Jet Algorithms in the ATLAS Detector, ATL-PHYS-INT-2010-129, CERN, Geneva, Dec, 2010.

[107] ATLAS Collaboration. https://twiki.cern.ch/twiki/bin/view/AtlasProtected/JetInputs.

[108] ATLAS Collaboration, Expected Performance of the ATLAS Experiment - Detector, Trigger and Physics: Jet Reconstruction Performance, arXiv:0901.0512 [hep-ex].

[109] D. E. Groom, Energy flow in a hadronic cascade: Application to hadron calorimetry, Nucl. Instrum. Meth. A 572 (2007) no. 2, 633 - 653.

[110] T. Gabriel, D. Groom, P. Job, N. Mokhov, and G. Stevenson, Energy dependence of hadronic activity, Nucl. Instrum. Meth. A 338 (1994) no. 2-3, 336 - 347.

[111] ATLAS Collaboration, Jet energy scale and its systematic uncertainty in proton-proton collisions at $\sqrt{s}=7 \mathrm{TeV}$ in ATLAS 2010 data, ATLAS-CONF-2011-032, CERN, Geneva, March, 2011.

[112] ATLAS Collaboration, Expected Performance of the ATLAS Experiment - Detector, Trigger and Physics, arXiv:0901.0512 [hep-ex].

[113] E. Bergeaas, C. Issever, K. Jon-And, K. Lohwasser, B. T. King, and D. Milstead, Local Hadronic Calibration of Single Pion Data from the Combined ATLAS Testbeam of 2004, ATL-COM-LARG-2007-010 (Internal Note), CERN, Geneva, Oct, 2007.

[114] T. Barillari et al., Local Hadronic Calibration, ATL-LARG-PUB-2009-001-2, CERN, Geneva, Jun, 2008.

[115] ATLAS Collaboration, Jet energy resolution and selection efficiency relative to track jets from in-situ techniques with the ATLAS Detector Using Proton-Proton Collisions at a Center of Mass Energy $\sqrt{s}=7 \mathrm{TeV}$, ATL-COM-PHYS-2010-054, CERN, Geneva, Jul, 2010.

[116] ATLAS Collaboration, Jet Energy Resolution from In-situ Techniques with the ATLAS 
Detector Using Proton-Proton Collisions at a Center of Mass Energy $\sqrt{s}=7 \mathrm{TeV}$, ATL-COM-PHYS-2011-240, CERN, Geneva, Mar, 2011.

[117] D $\emptyset$ Collaboration, B. Abbott et al., High-p $p_{T}$ jets in $p \bar{p}$ collisions at $\sqrt{s}=630 \mathrm{GeV}$ and 1800 GeV, Phys. Rev. D64 (2001) 032003, arXiv:hep-ex/0012046.

[118] UA2 Collaboration, P. Bagnaia et al., Measurement of Production and Properties of Jets at the CERN anti-p p Collider, Z. Phys. C20 (1983) 117-134.

[119] ATLAS Collaboration, Determination of the jet energy measurement uncertainty using photon-jet events in proton-proton collisions at $\sqrt{s}=7$ TeV, ATLAS-CONF-2011-031, CERN, Geneva, February, 2011.

[120] ATLAS Collaboration, Probing the jet energy measurement at the TeV-scale using the multi-jet balance technique in proton-proton collisions at $\sqrt{s}=7 \mathrm{TeV}$, ATLAS-CONF-2011-029, CERN, Geneva, February, 2011.

[121] ATLAS Collaboration, Determination of the ATLAS jet energy measurement uncertainties using tracks in proton proton collisions at $\sqrt{s}=7 \mathrm{TeV}$, ATLAS-CONF-2011-067, CERN, Geneva, May, 2011.

[122] ATLAS Collaboration, In-situ pseudorapidity intercalibration for evaluation of jet energy scale uncertainty using dijet events in proton-proton collisions at $\sqrt{s}=7 \mathrm{TeV}$, ATLAS-CONF-2011-014, CERN, Geneva, February, 2011.

[123] P. Z. Skands, Tuning Monte Carlo Generators: The Perugia Tunes, Phys. Rev. D 82 (2010) 074018, arXiv:1005.3457 [hep-ph].

[124] P. Francavilla. Private communication.

[125] ATLAS Collaboration, Jet energy scale and its systematic uncertainty for jets produced in proton-proton collisions at $\sqrt{s}=7 \mathrm{TeV}$ and measured with the ATLAS detector, ATLAS-CONF-2010-056, CERN, Geneva, July, 2010.

[126] ATLAS Collaboration, Update on the jet energy scale systematic uncertainty for jets produced in proton-proton collisions at $\sqrt{s}=7$ TeV measured with the ATLAS detector, ATLAS-CONF-2011-007, CERN, Geneva, February, 2011.

[127] D. Lopez Mateos, E. W. Hughes, and A. Schwartzman, A Simple $p_{T^{-}}$and $\eta$-Dependent Monte Carlo-Based Jet Calibration (Internal Note), ATL-PHYS-INT-2009-077, CERN, Geneva, Aug, 2009.

[128] ATLAS Collaboration, ATLAS Calorimeter Response to Single Isolated Hadrons and Estimation of the Calorimeter Jet Scale Uncertainty, ATLAS-CONF-2011-028, CERN, Geneva, March, 2011.

[129] E. Khramov et al., Study of the response of the hadronic barrel calorimeter in the ATLAS combined test-beam to pions of energies from 20-GeV to 350-GeV for beam impact points from 0.2 to 0.65, ATL-TILECAL-PUB-2009-007, CERN, Geneva, Apr, 2009.

[130] ATLAS Collaboration, Photon Conversions at $\sqrt{s}=900 \mathrm{GeV}$ measured with the ATLAS Detector, ATLAS-CONF-2010-007, CERN, Geneva, Jun, 2010.

[131] ATLAS Collaboration, Probing the material in front of the ATLAS electromagnetic calorimeter with energy flow from $\sqrt{s}=7 \mathrm{TeV}$ minimum bias events, 
ATLAS-CONF-2010-037, CERN, Geneva, Jul, 2010.

[132] ATLAS Collaboration, Study of Jet Shapes in Inclusive Jet Production in pp Collisions at $\sqrt{s}=7$ TeV using the ATLAS Detector, arXiv:1101.0070 [hep-ex].

[133] ATLAS Collaboration, ATLAS Monte Carlo tunes for MC09, ATL-PHYS-PUB-2010-002, CERN, Geneva, Mar, 2010.

[134] ATLAS Collaboration, Close-by Jet Effects on Jet Energy Scale Calibration in pp Collisions at $\sqrt{s}=/$ TeV with the ATLAS Detector, ATLAS-CONF-2011-062, CERN, Geneva, April, 2011.

[135] Light-quark and Gluon Jets in ATLAS, ATLAS-CONF-2011-053, CERN, Geneva, Apr, 2011.

[136] C. Doglioni, Dead material correlation studies [Internal talk at inclusive jet cross section meeting], https://indico.cern.ch/getFile.py/access?contribId=0\&resId= 2\&materialId=slides\&conf Id=134583, Apr, 2011.

[137] Measurement of inclusive jet and dijet cross sections in proton-proton collision data at 7 TeV centre-of-mass energy using the ATLAS detector, ATLAS-CONF-2011-047, CERN, Geneva, Mar, 2011.

[138] C. Cuenca Almenar, ATLAS Online Data Quality Monitoring, ATL-DAQ-PROC-2010-018, CERN, Geneva, Jul, 2010.

[139] P. Waller, ATLAS Data Quality Monitoring: Experience with First Collision Data, ATL-DAPR-PROC-2010-001, CERN, Geneva, Nov, 2010.

[140] ATLAS Collaboration, Data-Quality Requirements and Event Cleaning for Jets and Missing Transverse Energy Reconstruction with the ATLAS Detector in Proton-Proton Collisions at a Center-of-Mass Energy of $\sqrt{s}=7$ TeV , ATLAS-CONF-2010-038, CERN, Geneva, July, 2010.

[141] ATLAS Collaboration, ATLAS Collaboration, Charged-particle multiplicities in pp interactions at sqrt(s) $=900 \mathrm{GeV}$ measured with the ATLAS detector at the LHC, Phys. Lett. B688 (2010) 21-42, arXiv:1003.3124 [hep-ex].

[142] ATLAS Collaboration, Measurement of inclusive jet and dijet cross sections in proton-proton collision data at $7 \mathrm{TeV}$ centre-of-mass energy using the ATLAS detector (internal note), ATL-COM-PHYS-2011-738, CERN, Geneva, June, 2011.

[143] V. Lendermann, J. Haller, M. Herbst, K. Kruger, H.-C. Schultz-Coulon, et al., Combining Triggers in HEP Data Analysis, Nucl.Instrum.Meth. A604 (2009) 707-718, arXiv:0901.4118 [hep-ex].

[144] ATLAS Collaboration, Jet energy measurement with the ATLAS detector in proton-proton collisions at $\sqrt{s}=7 \mathrm{TeV}$ in 2010, ATLAS-PERF-2011-03-001 [Internal note], CERN, Geneva, In preparation, 2011.

[145] A. Nepomuceno, Z. Maxa, S. Boeser, and R. Teuscher, Visualizing and Understanding ATLAS Calorimeters with Atlantis, ATL-SOFT-PROC-2010-001, CERN, Geneva, Mar, 2010.

[146] G. Cowan, A survey of unfolding methods for particle physics, , Feb, 2002. 
[147] G. Choudalakis, Unfolding in ATLAS, , Jan, 2011.

[148] B. Malaescu, An Iterative, Dynamically Stabilized(IDS) Method of Data Unfolding, Proceedings of PhyStat 2011 (CERN) (2011), arXiv:1106.3107 [physics.data-an].

[149] B. Malaescu, An iterative, dynamically stabilized method of data unfolding, ArXiv e-prints (2009), arXiv:0907.3791 [physics.data-an].

[150] S. Alioli, K. Hamilton, P. Nason, C. Oleari, and E. Re, Jet pair production in POWHEG, arXiv:1012.3380 [hep-ph].

[151] H.-L. Lai et al., New parton distributions for collider physics, Phys. Rev. D82 (2010) 074024, arXiv:arXiv:1007.2241 [hep-ph].

[152] New ATLAS event generator tunes to 2010 data, ATL-PHYS-PUB-2011-008, CERN, Geneva, Apr, 2011.

[153] H.-L. Lai et al., Uncertainty induced by QCD coupling in the CTEQ global analysis of parton distributions, Phys. Rev. D82 (2010) 054021, arXiv:1004.4624 [hep-ph].

[154] ATLAS tunes of PYTHIA 6 and Pythia 8 for MC11, ATL-PHYS-PUB-2011-009, CERN, Geneva, Jul, 2011.

[155] A. Sherstnev and R. Thorne, Different PDF approximations useful for LO Monte Carlo generators, arXiv:0807.2132 [hep-ph].

[156] HEPData Collaboration. http://hepdata.cedar.ac.uk/.

[157] W. J. Stirling. Private communication. http://www.hep.phy.cam.ac.uk/ wjs/plots/plots.html.

[158] H1 and ZEUS Collaboration, V. Radescu, Combination and QCD analysis of the HERA inclusive cross sections, PoS ICHEP2010 (2010) 168.

http://pubdb.desy.de/fulltext/getfulltext.php?uid=13358-38069.

[159] S. Forte, Parton distributions at the dawn of the LHC, Acta Phys. Polon. B41 (2010) 2859-2920, arXiv:1011.5247 [hep-ph].

[160] A. D. Martin, W. J. Stirling, R. S. Thorne, and G. Watt, Parton distributions for the LHC, Eur. Phys. J. C63 (2009) 189-285, arXiv:0901.0002 [hep-ph].

[161] R. D. Ball et al., A first unbiased global NLO determination of parton distributions and their uncertainties, Nucl. Phys. B838 (2010) 136-206, arXiv:arXiv:1002.4407 [hep-ph].

[162] ATLAS Collaboration, Comparisons of the measured inclusive jet cross section to a selection of NLO QCD predictions, and a study of the parton content of the calculations, ATL-PHYS-PUB-2011-005, CERN, Geneva, Apr, 2011.

[163] M. Botje, Error estimates on parton density distributions, J. Phys. G28 (2002) 779-790, arXiv:0110123 [hep-ph].

[164] D. Stump et al., Uncertainties of predictions from parton distribution functions. 1. The Lagrange multiplier method, Phys. Rev. D65 (2001) 014012, arXiv:0101051 [hep-ph].

[165] H1 Collaboration, Measurement of the Inclusive ep Scattering Cross Sections at Low $Q^{2}$ 
and $x$ at HERA, Eur.Phys.J. C63 (2009) 625-678, arXiv:0904.0929 [hep-ex].

[166] ZEUS Collaboration, A. Cooper-Sarkar. https://indico.cern.ch/getFile.py/ access? contribId=14\&sessionId=4\&res Id=1\&material Id=slides\&conf Id=97834.

[167] CHORUS Collaboration Collaboration, A. Kayis-Topaksu et al., Leading order analysis of neutrino induced dimuon events in the CHORUS experiment, Nucl.Phys. B798 (2008) 1-16, arXiv:0804.1869 [hep-ex].

[168] Particle Data Group Collaboration, C. Amsler et al., Review of particle physics, Phys. Lett. B667 (2008) 1-1340.

[169] R. Thorne and R. Roberts, An Ordered analysis of heavy flavor production in deep inelastic scattering, Phys.Rev. D57 (1998) 6871-6898, arXiv:9709442 [hep-ph].

[170] R. Thorne, A Variable-flavor number scheme for NNLO, Phys.Rev. D73 (2006) 054019, arXiv:0601245 [hep-ph].

[171] M. Botje, QCDNUM: Fast QCD Evolution and Convolution, Comput. Phys. Commun. 182 (2011) 490-532, arXiv:1005.1481 [hep-ph].

[172] J. Collins, Renormalization: An Introduction to Renormalization, the Renormalization Group and the Operator-Product Expansion. Cambridge University Press, 1984.

[173] H. Flacher et al., Revisiting the Global Electroweak Fit of the Standard Model and Beyond with Gfitter, Eur. Phys. J. C60 (2009) 543-583, arXiv:0811.0009 [hep-ph]. [Erratum-ibid.C71:1718,2011].

[174] GFitter Collaboration, Results for the Global Electroweak Standard Model Fit, including latest results from July 2011 (EPS11), http://gfitter.desy.de/Standard_Model/.

[175] H1 and Z. collaborations, QCD analysis and determination of $\alpha_{s}$ using the combined H1 and ZEUS inclusive cross sections together with the jet production cross section measured by the H1 and the ZEUS experiments, H1prelim-11-034, ZEUS-prel-11-001, DESY, Hamburg, Nov, 2010.

[176] S. Whitehead, A Measurement of the $W$ Boson Charge Asymmetry with the ATLAS Detector. PhD thesis, 2011.

[177] G. Soyez, A Simple description of jet cross-section ratios, Phys.Lett. B698 (2011) 59-62, arXiv:1101.2665 [hep-ph].

[178] M. Dasgupta, L. Magnea, and G. P. Salam, Non-perturbative QCD effects in jets at hadron colliders, JHEP 02 (2008) 055, arXiv:0712.3014 [hep-ph].

[179] S. Shimizu, E. Fullana, E. Feng, P. Francavilla, and P. Starovoitov, Distribution of jet pt at proton-proton collisions with 2.76 TeV and $7 \mathrm{TeV}$ center of mass energy in 2011, ATL-COM-PHYS-2011-1082, CERN, Geneva, Aug, 2011.

https://twiki.cern.ch/twiki/bin/view/AtlasPublic/

StandardModelPublicCollisionPlots\#Jet_production.

[180] C. Doglioni, P. Francavilla, P. Loch, K. Perez, and R. Vitillo, Design of the Jet Performance Software for the ATLAS Experiment at LHC, ATL-SOFT-PROC-2011-037, CERN, Geneva, Feb, 2011. 
[181] https://twiki.cern.ch/twiki/bin/view/AtlasProtected/JetPerformance.

[182] I. Berg, muParser - a fast math parser library, Http://muparser.sourceforge.net/index.html.

[183] B. Andersson, G. Gustafson, and B. Nilsson-Almqvist, A model for low- $p$ T hadronic reactions with generalizations to hadron-nucleus and nucleus-nucleus collisions, Nucl. Phys. B 281 (1987) no. 1-2, 289 - 309.

[184] P. Z. Skands, The Perugia Tunes, arXiv:0905.3418 [hep-ph].

[185] A. Buckley, H. Hoeth, H. Lacker, H. Schulz, and J. E. von Seggern, Systematic event generator tuning for the LHC, Eur. Phys. J. C 65 (2010) 331-357, arXiv:0907. 2973 [hep-ph]. 


\section{Appendix A.}

\section{In-situ validation of the jet energy scale and resolution}

This appendix contains additional details on the techniques used to validate the jet energy scale and jet energy resolution in-situ. The jet energy resolution techniques are described in Section A.1, while the techniques to validate the Monte Carlo based jet energy scale are in Section A.2.

\section{A.1. Estimate of the jet energy resolution with data}

\section{Dijet balance method}

The dijet balance method [117] relies on the energy conservation in the transverse plane in the case of dijet events. Events with such topology are selected applying a cut on the azimuthal angle between the two leading jets $\Delta \phi$ so that they are back to back $(\Delta \phi<2.8)$ and limiting the presence of significant third jet radiation by imposing $p_{T, 3}^{E M}<10 \mathrm{GeV}$, where $p_{T, 3}^{E M}$ is the third jet EM-scale transverse momentum. The two jets are required to be in the same rapidity region so that their energy will be comparable and they will be traversing similar calorimeter technologies $^{1}$. The asymmetry $\mathcal{A}$ between the two jets is defined as:

$$
A=\frac{p_{T}^{1}-p_{T}^{2}}{p_{T}^{1}+p_{T}^{2}} .
$$

Taking the variance of the asymmetry and assuming that the transverse momenta of the two jets are balanced $\left(p_{T}^{1} \approx p_{T}^{2}\right)$ leads to a direct connection between the width of the asymmetry distribution and that of the jet transverse momentum:

\footnotetext{
${ }^{1} \mathrm{~A}$ similar procedure can also be used to estimate the resolution of jets in different rapidity regions, if the resolution for one of the two rapidity regions is known.
} 


$$
\frac{\sigma_{p_{T}}}{p_{T}}=\sqrt{2} \sigma_{A}
$$

Since the cut on the $p_{T}$ of the third jet cut does not exclude the presence of additional jets with $p_{T, 3}^{E M}<10 \mathrm{GeV}$ that could imbalance the event, a soft radiation correction $K_{\text {soft }}$ is derived by extrapolating the fractional resolution to a value of $p_{T, 3}^{E M}<0$. This is done employing a series of steps in the $p_{T, 3}^{E M}$ cut and using a linear fit for the extrapolation, as shown in Figure A.1. The correction is taken as the ratio of the extrapolated fractional resolution with respect to the fractional resolution with the $p_{T, 3}^{E M}<10 \mathrm{GeV}$ cut:

$$
K_{\text {soft }}\left(p_{T}\right)=\frac{\left(\frac{\sigma_{p_{T}}}{p_{T}}\right)_{p_{T, 3}^{E M} \rightarrow 0 G e V}}{\left(\frac{\sigma_{p_{T}}}{p_{T}}\right) p_{T, 3}^{E M}<10 G e V}
$$

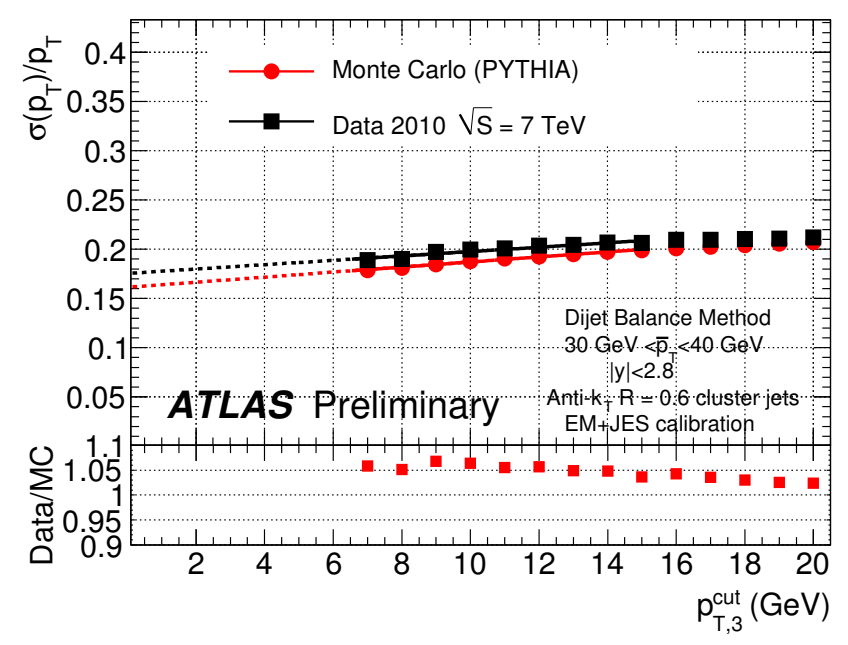

Figure A.1.:Fractional resolution as a function of the value of the cut on the third jet $p_{T}$, together with the linear fit (solid line) and extrapolation (dashed line) to $p_{T, 3}^{E M}=0$ in a sample $p_{\mathrm{T}}^{\mathrm{avg}}$ bin. Figure from [115]

The correction is then fitted as a function of $p_{T}$ using the empirical parameterisation $K_{\text {soft }}\left(p_{T}\right)=a+b / \log ^{2}\left(p_{T}\right)$, which has the desired property of becoming unity for large values of $p_{T}$ (where radiation is negligible with respect to the jet momenta). Additional parameterisations were employed, and the variation of the corrected resolution is taken as a systematic uncertainty of the method of up to $5 \%$.

The imbalance between calorimeter jets due to physics effects is also removed in order to only include calorimeter effects in the evaluation of the fractional resolution. For this reason, the particle-level imbalance is derived using Monte Carlo truth jets using the method above and subtracted in quadrature from the in-situ resolution after the soft radiation correction. 
The total systematic uncertainty for the dijet balance method amounts to $4-6 \%$ in the $p_{\mathrm{T}}^{\text {jet }}$ range of $20-500 \mathrm{GeV}$.

\section{Bisector method}

The bisector method [118] uses the decomposition of the vector sum of the two leading jet four momenta $\mathbf{P}_{\mathbf{T}}$ to estimate the jet energy resolution. As shown in Figure A.2, $\mathbf{P}_{\mathbf{T}}$ is projected on the $\eta$ axis (which bisects the angle $\phi_{1,2}$ between the two jets) and the orthogonal $\psi$ axis. $\mathbf{P}_{\mathbf{T}}$ represents the imbalance between the transverse momenta of the two jets. Its two projections on the $\eta, \psi$ axes are called $p_{T, \eta}$ and $p_{T, \psi}$ and their variance is used to estimate the jet resolution.

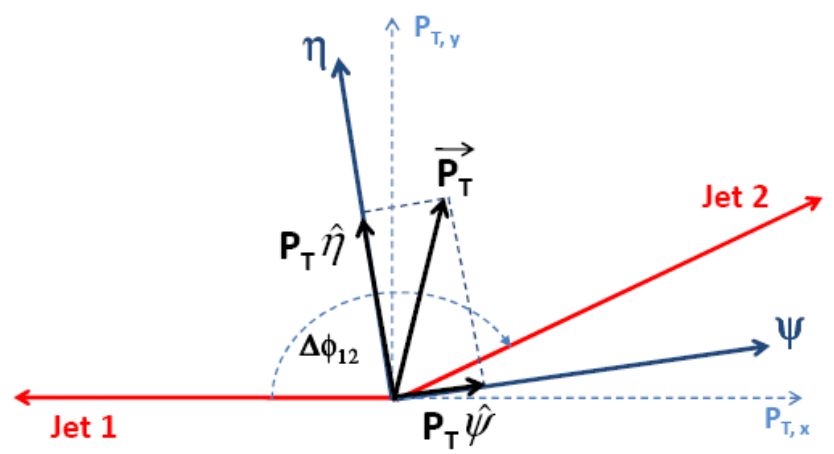

Figure A.2.:Schematic representation of the decomposition of the vector sum of the two leading jet four momenta used to estimate the jet resolution using the bisector method. Figure from [115].

The underlying assumption is that at particle level, the two components of the imbalance vector will have equal fluctuations, and the imbalances are due to fluctuations from calorimeter effects. Using this assumption, the fractional resolution can be expressed in terms of the variances of the two imbalance components induced by calorimeter effects $\left(\sigma_{\eta}^{2, \text { calo }}\right.$ and $\left.\sigma_{\psi}^{2, \text { calo }}\right)$ and the angle between the two jets:

$$
\frac{\sigma_{p_{T}}}{p_{T}}=\frac{\sqrt{\sigma_{\eta}^{2, \text { calo }}-\sigma_{\psi}^{2, \text { calo }}}}{p_{T} \sqrt{2} \cos \left(\Delta \phi_{1,2}\right)}
$$

The variances $\sigma_{\eta}^{2, \text { calo }}$ and $\sigma_{\psi}^{2, \text { calo }}$ are extracted from the distribution of the projections of $\mathbf{P}_{\mathbf{T}}$ in bins of the average $p_{\mathrm{T}}^{\text {jet }}\left(p_{\mathrm{T}}^{\text {avg }}\right)$ of the two leading jets.

The event selection follows that of the dijet balance method, but removing the cut on the angle between the two jets. The assumption of isotropy of the soft radiation is tested by changing the value of the third jet cut and checking $\sigma_{\eta}^{2 \text {,calo }}, \sigma_{\psi}^{2 \text {,calo }}$ and their difference. As shown in Figure A.3, the increase of the soft radiation contribution cancels in the squared difference 
within $3-4 \%$ for the $30-40 \mathrm{GeV} p_{\mathrm{T}}^{\text {avg }}$ bin. The precision to which this assumption holds is taken as a systematic uncertainty of the method.

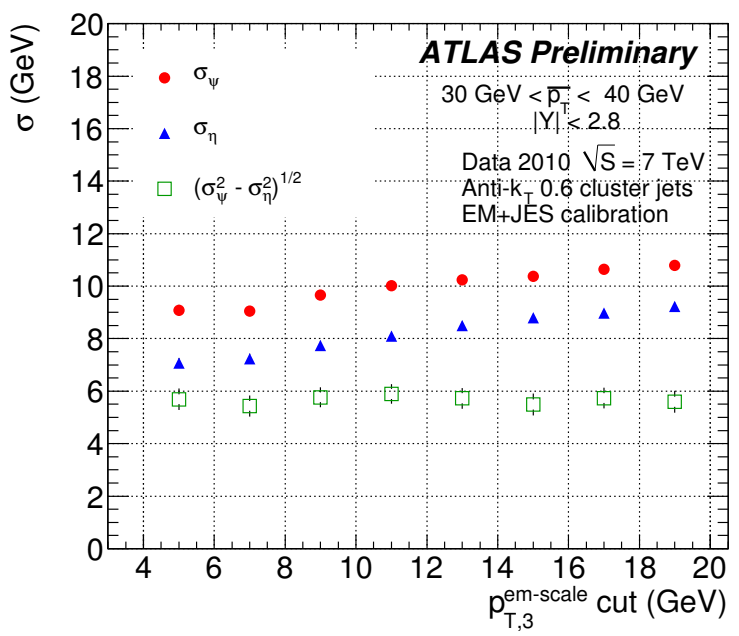

Figure A.3.:Variances $\sigma_{\eta}^{2, \text { calo }}, \sigma_{\psi}^{2, \text { calo }}$ and their difference as a function of the third jet $p_{T, 3}^{E M}$ cut in a sample $p_{\mathrm{T}}^{\text {avg }}$ bin. Figure from [115].

The total systematic uncertainty for the bisector method amounts to $3-4 \%$ in the $p_{\mathrm{T}}^{\text {jet }}$ range of $20-500 \mathrm{GeV}$.

\section{A.2. In-situ validation of the jet energy scale}

\section{A.2.1. Photon-jet balance}

The transverse momentum balance between a photon and the hadronic recoil is used to probe the jet energy scale in two methods with different systematic uncertainties and sensitivities [119].

The direct gamma-jet balance exploits the balance between photon and jet transverse momenta that is found at leading order of perturbation theory. The jet response is defined as the ratio of the photon and jet transverse momenta: $p_{T}^{\gamma} / p_{\mathrm{T}}^{\mathrm{jet}}$. Cuts on the azimuthal distance between the photon and the jet and on the subleading jet are applied to to suppress soft radiation and multi-jet configurations. Good photons and matching jets are selected within a fiducial calorimeter region. Jet faking photons are rejected using an isolation cut.

The Missing-energy Projection Fraction (MPF) method probes the hadronic response using the leading-order transverse momentum conservation between the photon $\left(p_{T}^{\gamma}\right)$ and its full hadronic recoil $\left(p_{\mathrm{T}}^{\text {jet }}\right): \mathbf{p}_{T}^{\gamma}+\mathbf{p}_{\mathrm{T}}^{\text {jet }}=0$.

This method assumes that the imbalance between the jet and photon transverse momenta measured in the calorimeters is generated by calorimeter non compensation, noise suppression effects and inactive material in front of the calorimeters (dead material). The imbalance vector 
can written in terms of the missing energy estimated from the calorimeter measurement $\mathbf{E}_{\mathbf{T}}^{\text {miss }}$ and it can be written in terms of the jet and photon calorimeter responses $\mathcal{R}_{\text {jet }}$ and $\mathcal{R}_{\gamma}{ }^{2}$ :

$$
\mathcal{R}_{\gamma} \mathbf{p}_{\mathbf{T}}^{\gamma}+\mathcal{R}_{\text {jet }} \mathbf{p}_{\mathrm{T}}^{\mathrm{jet}}=-\mathbf{E}_{\mathrm{T}}^{\mathrm{miss}}
$$

If the photon is well calibrated, its response will be unitary: exploiting momentum balance and projecting the missing energy vector along the photon direction, the above equation can be rewritten as:

$$
R_{\mathrm{jet}}=1+\frac{\mathbf{n}^{\gamma} \cdot \mathbf{E}_{\mathrm{T}}^{\mathrm{miss}}}{\left|\mathbf{p}_{\mathrm{T}}^{\gamma}\right|}
$$

In Equation A.3, the jet response is written in terms of calorimeter observables, and does not depend directly on the jet transverse momentum: it is therefore roughly independent of the jet algorithm used for jet reconstruction. The same kinematic cuts mentioned for the direct gamma-jet balance are also used in the MPF analysis.

The jet energy scale is validated using the jet response with respect to the photon in data and Monte Carlo before and after the EM+JES calibration. The MPF method does not depend directly on the jet transverse momentum. Therefore a simple shift of the energy scale, as in the case of the EM+JES calibration, will not affect the data/Monte Carlo ratio. Therefore the validation of the EM+JES calibration can be performed using jets at the electromagnetic scale.

Results for the EM+JES calibration are shown in Figures A.4 (a) and (b) for the direct balance method and the MPF method respectively, and results for Global and Local Cell Weighting can be found in Reference [119].

The jet energy scale is validated with the photon-jet balance method for all calibrations considered to within $5 \%$ for $p_{T}^{\gamma}<45 \mathrm{GeV}$ and to within $3 \%$ for $45<p_{T}^{\gamma}<250 \mathrm{GeV}$. The major systematics, related to the photon energy scale, the presence of jets faking photons, the soft radiation and the in-time pile-up have also been considered in Reference [119] and found to be less than $1.6 \%$ for $p_{T}^{\gamma}>45 \mathrm{GeV}$.

\section{A.2.2. Balance between a high- $p_{T}$ jet recoiling against lower- $p_{T}$ jets}

The calibration of very high transverse momentum jets is a particularly challenging topic for the first ATLAS data due to the limited statistics available for in-situ validations with $\gamma$-jet methods. A well understood energy scale is necessary for highly energetic jets, since they are among the signatures for early New Physics searches. When jets at low transverse momentum are reasonably well calibrated (e.g. using the photon-jet balance methods), they can be used to assess the calibration of the recoiling, high- $p_{T}$ jet using transverse momentum balance, as

\footnotetext{
${ }^{2}$ The photon response is defined as the ratio of the photon $p_{T}$ measured in the calorimeter and the true photon $p_{T}$.
} 


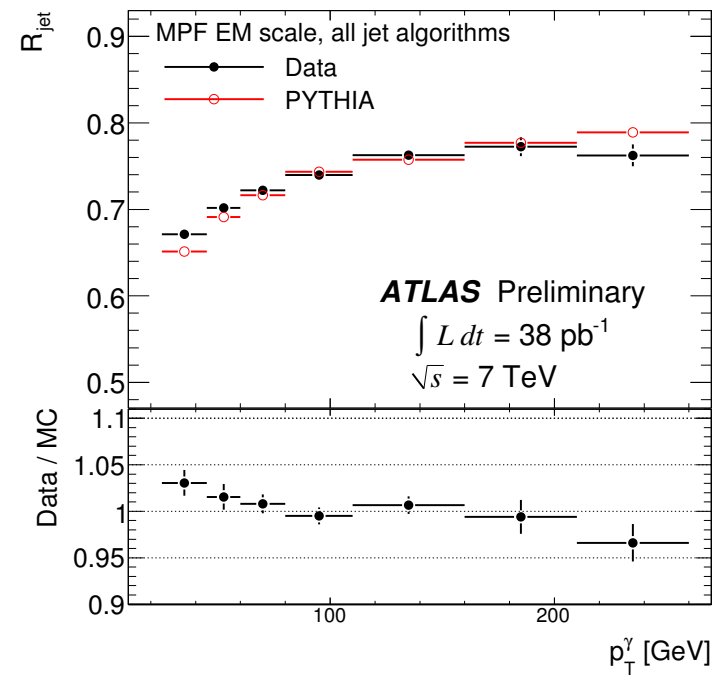

(a)

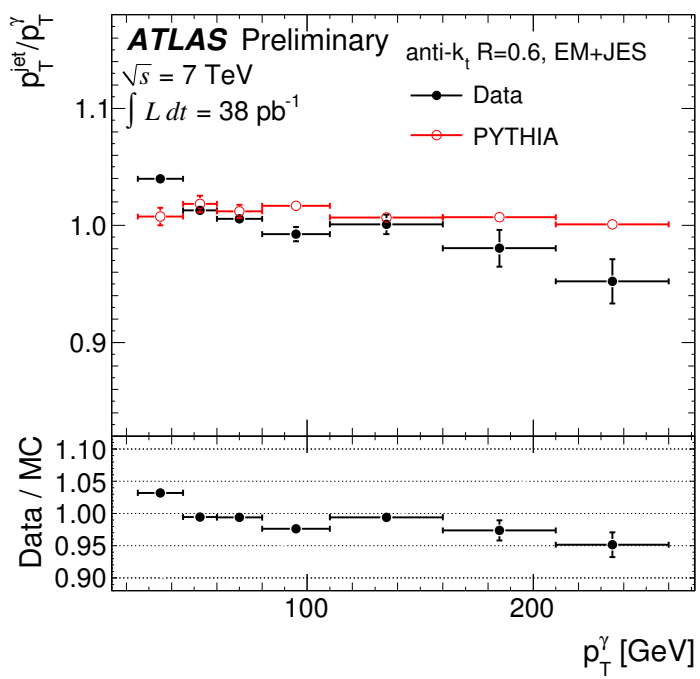

(b)

Figure A.4.:Validation of the EM+JES scale calibration using the MPF method (left) and gamma-jet direct balance (right).

detailed in [120]. The Multi-Jet Balance (MJB in the following) is defined as the ratio between the leading jet $p_{T}^{\text {Leading }}$ and the vector sum of the transverse momenta of all recoiling jets $p_{T}^{\text {Recoil }}$. The data/Monte Carlo ratio of MJB is used to validate the jet energy scale in 2010 data up to the Terascale. A selection of events with a leading jet and a well defined recoil system that is not dominated by a single jet are selected using cuts on the azimuthal angle between the jet and the recoil system, the isolation of the leading jet and the transverse momentum of the subleading jet in the recoil system.

The Multi-Jet Balance method validates the energy scale to within 3\%, as shown in Fig. A.5. The systematics of the method are estimated in [120]: they take into account the jet energy scale uncertainty, the dependence of the jet response on the jet flavour and on the presence of close-by jets in the recoil system (discussed in detail in Chapter 5), the analysis cuts, soft QCD effects and pile-up. These effects and are estimated to lead to a systematic uncertainty of about $4 \%$.

\section{A.2.3. Comparison of calorimeter to track jet transverse momentum}

Another method of validating the jet energy scale is the comparison of the transverse momentum of the calibrated jets measured with the calorimeter to associated jets measured in an independent subsystem, as in the case of the track-jet measurement presented in [121]. Track-jets are composed by all charged tracks measured in the ATLAS inner detector within an $\eta, \phi$ cone equal to the distance parameter of the jet considered. The transverse momentum of track-jets is compared to the $p_{T}$ of calibrated calorimeter jets. The double ratio $R_{r_{t r k}}$ of the track-jet and calorimeter jet transverse momenta in data and Monte Carlo is used for the validation of the jet energy scale uncertainty. Only jets with with a transverse above a $p_{T}$ dependent cut are used in the 


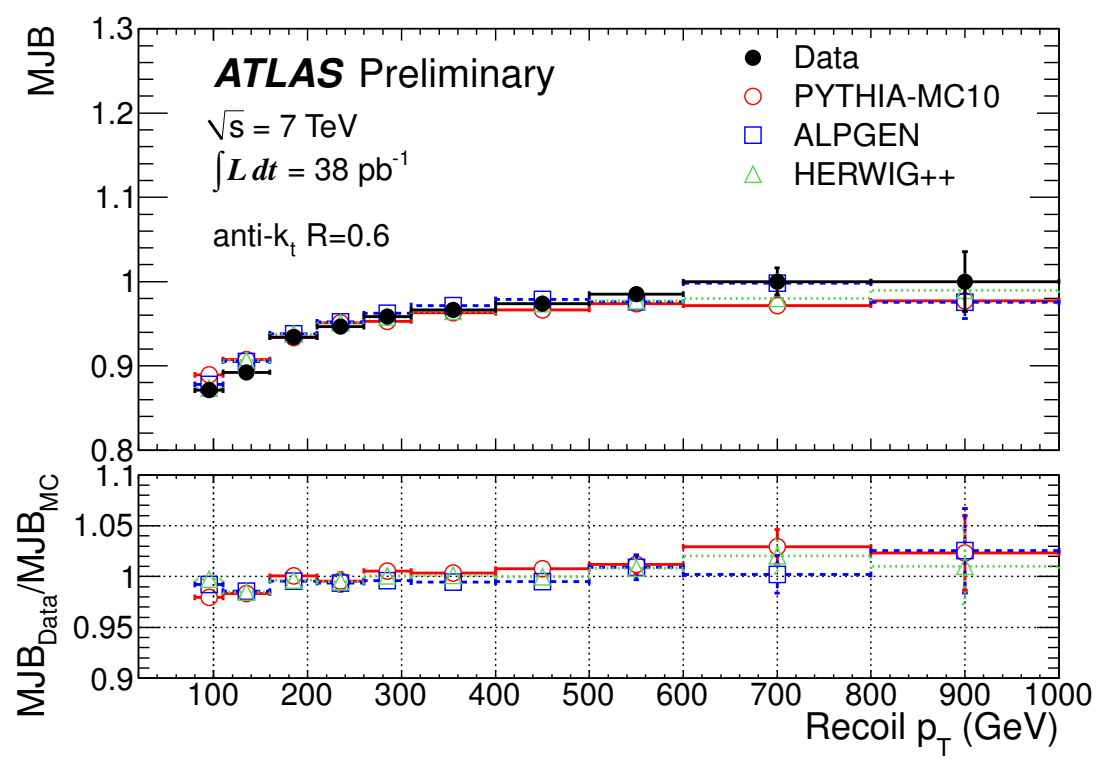

Figure A.5.:Validation of the EM+JES scale calibration using the Multi-Jet Balance technique.

measurement, to ensure close to full efficiency for the trigger used for measuring the jet. A cut on the jet pseudorapidity of less than 2.1 is needed to ensure that the majority of tracks belonging to the track-jet is found in the fiducial volume of the inner detector. Furthermore, in configurations where two jets are separated by a distance $\Delta R$ less than twice the jet distance parameter, only the hardest jets are used in order to minimize effects due to nearby jets on the measurement. The track selections are detailed in Section 4 of [121].

The double ratio $R_{r_{t r k}}$ for EM+JES calibrated jets in the central region is shown in Fig. A.6: data and simulation agree to better than $2 \%$ for jets in the $p_{T}$ range of 25 to $1000 \mathrm{GeV}$. The systematic uncertainties as estimated in [121] are related to the generator model dependence, the description of the material and the alignment in the inner detector, the tracking efficiency, and the calorimeter jet resolution, and they amount to less than $4 \%$. 


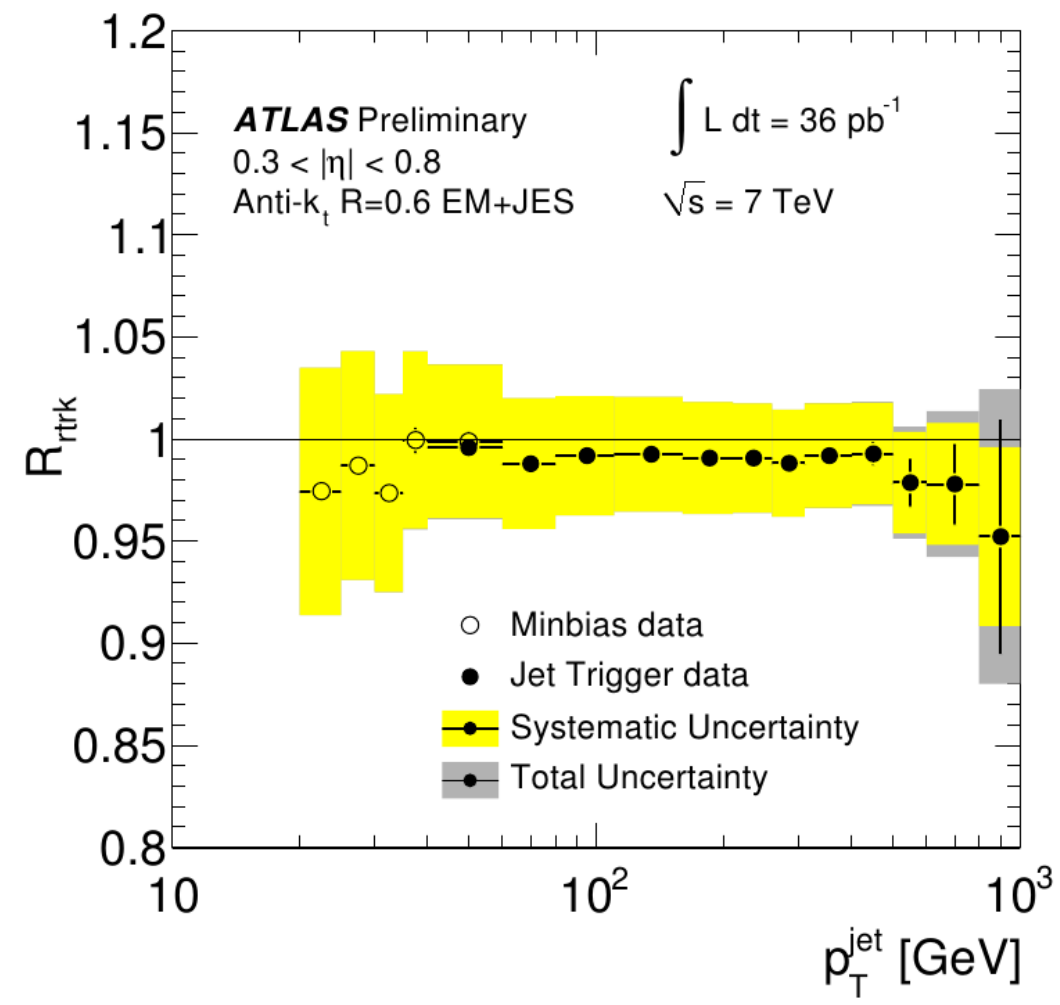

Figure A.6.:Validation of the EM+JES scale calibration using the double ratio of the track-jet and calorimeter jet transverse momenta in data and Monte Carlo. 


\section{Appendix B.}

\section{Jet performance analysis tools}

The goal of the JETPERFORMANCE package $[180,181]$ is to provide a common set of definitions and algorithms to measure quantities relevant to the performance of jet reconstruction through a series of standardised validation plots. Comparison and cross checking of jet reconstruction procedures on different samples are more effective if the individual users adopt a coherent approach, through code which is available within the ATHENA framework. While providing standardised ways for measuring the jet performance, this package is still easily extensible by the addition of user-defined tools and plots.

The design of the package and its use in combination with the JETFrAMEWORKTOOLS allows to perform cuts on the signal sample and define the benchmark quantities to be plotted at run-time. This is particularly convenient when the same analysis needs to be repeated multiple times with similar parameters, as in the case of the derivation of the JES uncertainty.

This Appendix starts with an overview of the JETFRAMEwORKToOLS package in Section B.1. The JETPERFORMANCE package, together with the workflow of a typical performance analysis is briefly described in Section B.2. Cases where the JeTPERFormance package has been employed are discussed in Section B.3.

\section{B.1. The JetFrameworkTools package}

A typical jet performance analysis takes as input a jet collection. Different analyses need to perform similar cuts, but with variable cut ranges: the need of a flexible framework with a uniform behaviour has been met with the design of the JETFRAMEWORKTOOLS package in the ATHENA software framework.

The JETFRAMEwORKTOOLS package provides the underlying infrastructure to steer workflows in the form of a Data Flow Graph (DFG). Each node of the graph, performs a specific action such as the retrieval of the object from memory, a selection cut or a plotting task. Graph nodes are called filters and selectors depending on their behaviour, and they can be concatenated. The graph then takes care of executing the actions (e.g. cuts, plotting) in each node in the correct order specified by the user.

Filters receive one or more collections of objects as input, and deliver a copy of one of the collections or a subset of it as output, while selectors perform a selection on the whole 
event. The most common use of the filters is to perform a selection on the input collections for example, a JetFilter will remove from the input collection all the jets that do not pass a user-defined kinematic cut.

The JETFRAMEWORKTOOLS package makes use of an external parser called MUPARSER [182] to allow definition of the selection variables and cut ranges at run time.

\section{B.2. The JetPerformance package}

The JetPerformance package is part of the ATHENA framework and it aims to provide a common infrastructure to evaluate the performance of the jet reconstruction (comparing e.g. calibrations, jet algorithms, different datasets).This is achieved by comparing the reconstructed jets to a collection of reference objects that can provide either in-situ balance or provide a truth benchmark such as particle jets built from MonteCarlo. The performance of the jet reconstruction is then measured in term of linearity, resolution, spatial resolution, purity and efficiency (where the latter three are available only for $\mathrm{MC}$ comparison) in kinematic and spatial bins.

This package aims to provide a common set of definitions and algorithms to measure quantities relevant to the performance of jet reconstruction through a series of standardised validation plots, compiled with the help the different JetPerformanceTools that take as input different collections of reference objects. Comparison and cross checking of jet reconstruction procedures on different samples are more effective if the individual users adopt a coherent approach, through code which is available within the ATHENA framework and commonly agreed algorithms which are customizable at run-time. At the same time, this package is still easily extensible by adding new user-defined Tools or performance plots.

The code structure has been developed to provide a common interface for performing the cuts for all the JetPerfTools, increased flexibility in the plotting from an intermediate data format and reduced code duplication (all the common characteristics and functions for the single tools are either abstracted in a base class or taken from centralised helper classes in the package).

\section{B.2.1. Jet Performance workflow}

The workflow of the JETPERFORMANCE software is composed of three steps:

1. the calculation of the relevant performance quantities on the selected signal is obtained by running the various JeTPERFoRMANCE tools on the input data. These tools extract basic quantities for measuring the jet performance and make them available for further analysis through a basic ROOT ntuple (intermediate data);

2. the intermediate data can be merged - this can be the case when the code is run in parallel with a distributed analysis framework or when output from different energy/trigger samples needs to be weighted and merged; 
3. the desired performance plots are obtained by running standardised algorithms on the (merged) output of the tools - calculations of the quantities relevant to the evaluation of the jet performance are performed and the quantities are plotted against the desired kinematic or spatial variable.

A performance plotting tool is a filter as described in Section B.1, since it takes as input a collection of reconstructed jets and a collection of reference objects. While leaving the input collections untouched, it will retrieve the relevant variables and save them into the intermediate data format for subsequent plotting. This is how the JeTPERFORMANCE code structure fits in the JetFrameworkTools framework.

An example of the workflow of a typical performance analysis that compares the kinematic properties of reconstructed jets to those of true jets is shown in figure B.1. Filters are used to perform the selection of the jets that will be used in the specific JetPerformance algorithm, in this case called JetPerfToolTruth. As a first step, both the true and the reconstructed jet collections are retrieved from the transient data storage through SourceFilters. A number of JetFilters can then be used to select only the jets of interest from the two jet collections, and only these jets will proceed to be analysed by the performance algorithm of choice. The output of the jet performance algorithm is an intermediate data structure (JetPerfDataStructure). The JetPerfDataStructure is subsequently read in another algorithm, called JetPerfPlottingAlg, where the final performance plots are produced.

\section{B.3. Use cases}

The performance of the jet reconstruction is evaluated in the JETPERFORMANCE package using in-situ energy balance techniques, as well as comparison to truth jets built from Monte-Carlo particles. The jet reconstruction performance is measured in terms of linearity, resolution, purity and efficiency in kinematic and spatial bins. Typical use cases are the comparison of different jet algorithms or calibrations, the validation of calibration constants and weights. The JETPERFORMANCE package is used in the ATLAS Jet-EtMiss working group for these purposes.

\section{B.3.1. Use case: validation of the jet energy scale calibrations}

The results presented in this section have been obtained using the JETPERFTOOLTRUTH tool: this tool evaluates the response of reconstructed jets by comparison with true jets, formed by applying the same jet algorithm to stable particles in Monte-Carlo data. This tool can be used for evaluating the energy response after the calibration and detector corrections. In order to consider a reconstructed and a true jet as generated by the same parton, a geometrical matching is performed - if the $\eta-\phi$ distance between the two jets is below a cut value specified by the user, the jets will be considered matched. If two jets are matched, their energies $E_{\text {reco }}$ and $E_{\text {true }}$ will be compared as a ratio in the response plot. A gaussian fit of the reconstructed energies of matched jets in a given phase space bin gives a mean value and spread for the reconstructed energy that is entered in the resolution plot. 


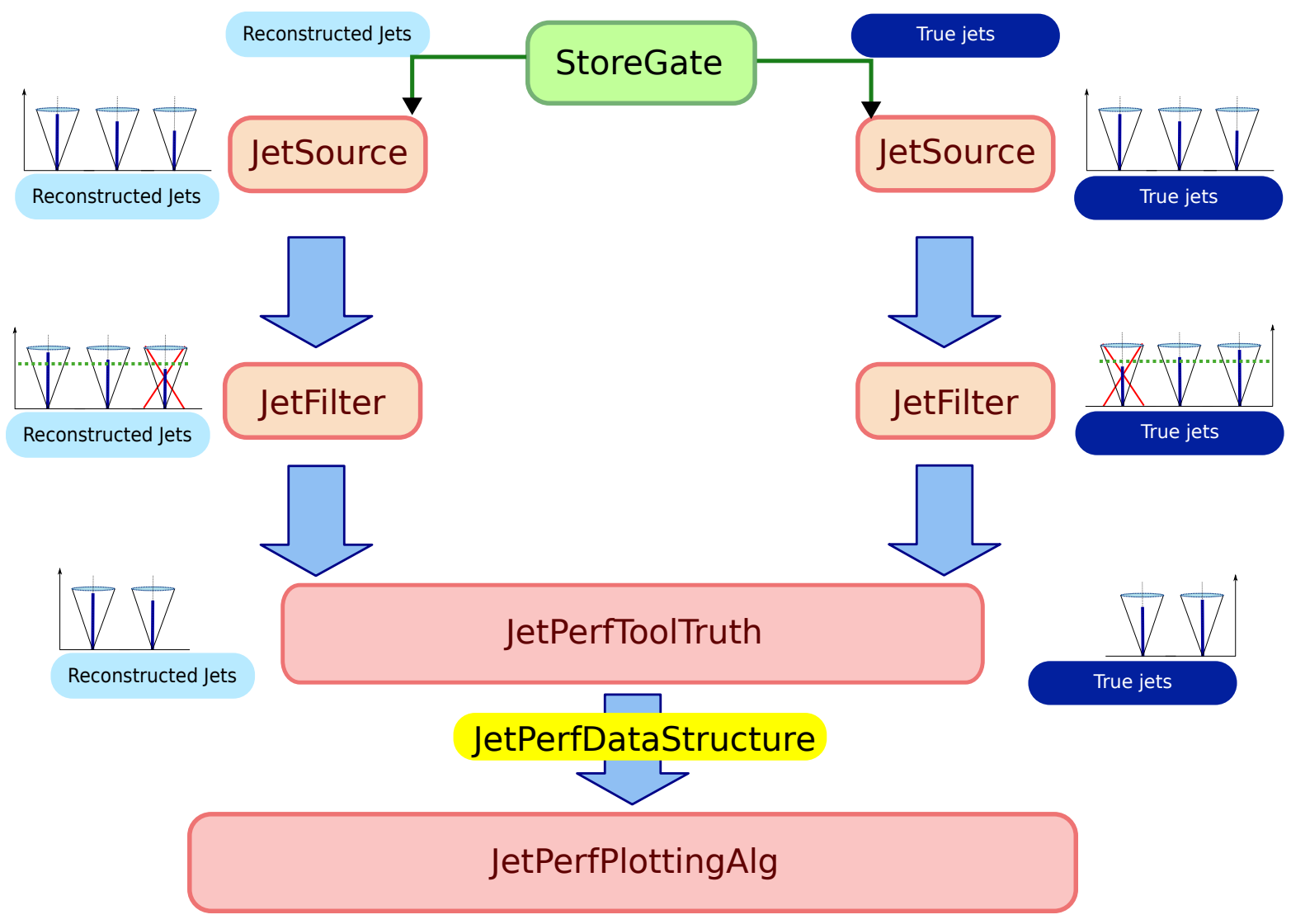

Figure B.1.:Example workflow of a typical jet performance analysis.

\section{B.3.2. Use case: jet energy scale uncertainty in ATLAS}

It is through the JetPerformance software, and the underlying DFG based framework, that the first estimate of the uncertainty on the jet energy scale for ATLAS has been derived [125]. This analysis fully exploited the flexibility of the software: The estimate of the jet response, performed in a similar manner as outlined above, needed to be repeated with the same cut flow for a number of Monte Carlo simulated samples with systematic variations applied in order to estimate the effects of those variations on the jet energy calibration. 


\section{Appendix C.}

\section{JES uncertainty plots}

This section contains the JES uncertainty summary plots of Chapter 5 for both jet distance parameters and for all $\eta$ regions.

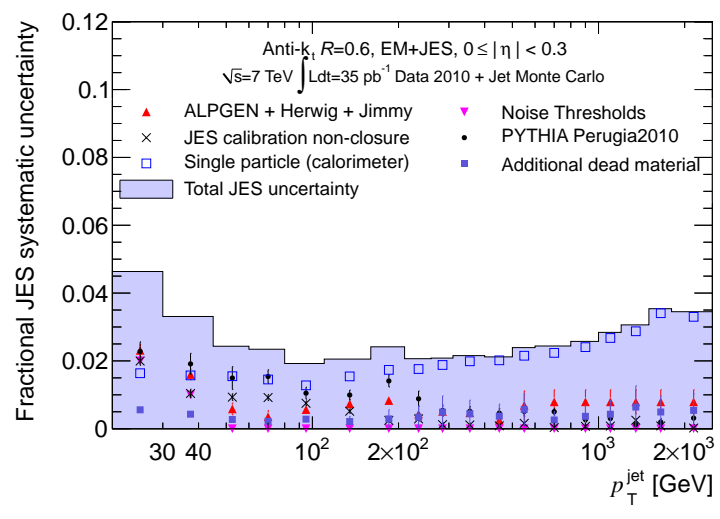

(a)

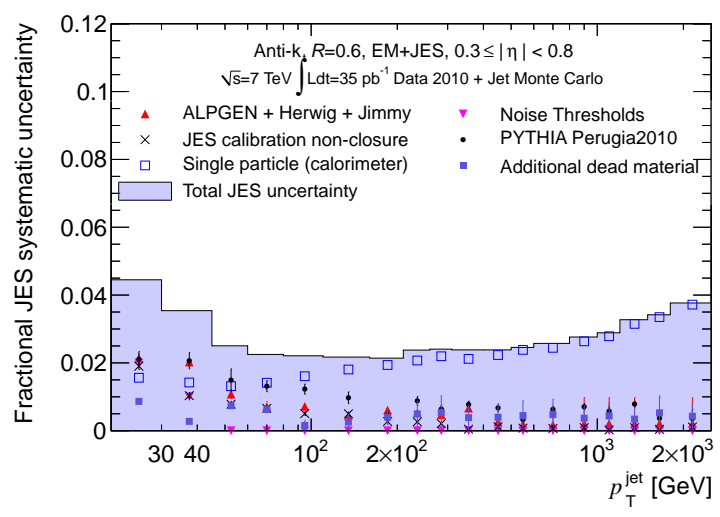

(b)

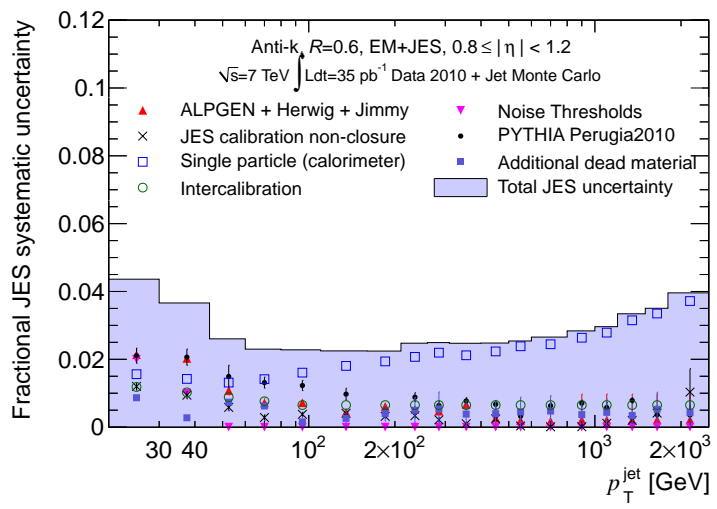

(c)

Figure C.1.:Fractional jet energy scale systematic uncertainty as a function of $p_{\mathrm{T}}^{\text {jet }}$ for jets with distance parameter $\mathrm{R}=0.6$ in the pseudorapidity regions within $0 \leq|\eta|<1.2$. The total uncertainty is shown as the solid light blue area. The individual sources are also shown, with uncertainties from the fit if applicable. 


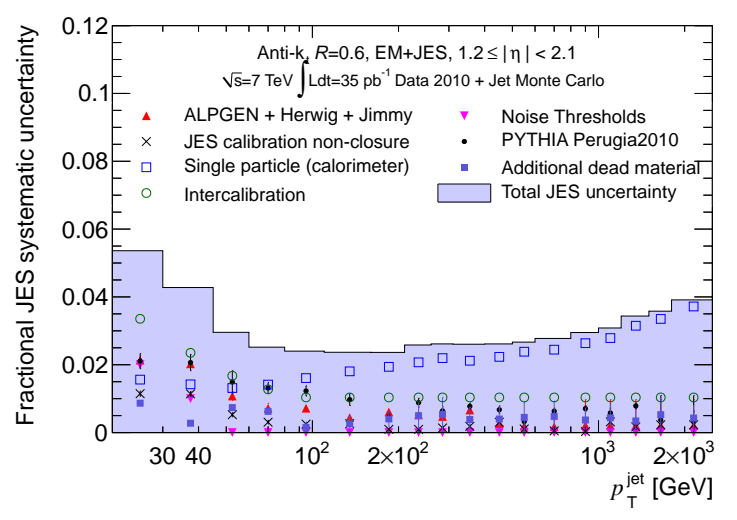

(a)

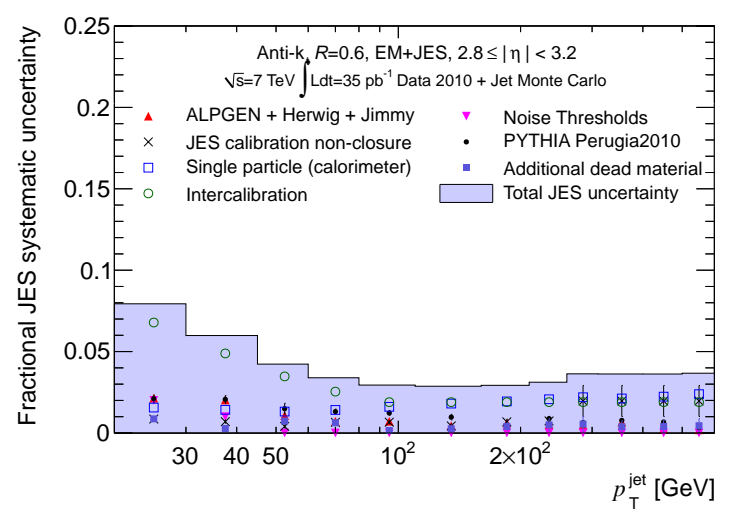

(c)

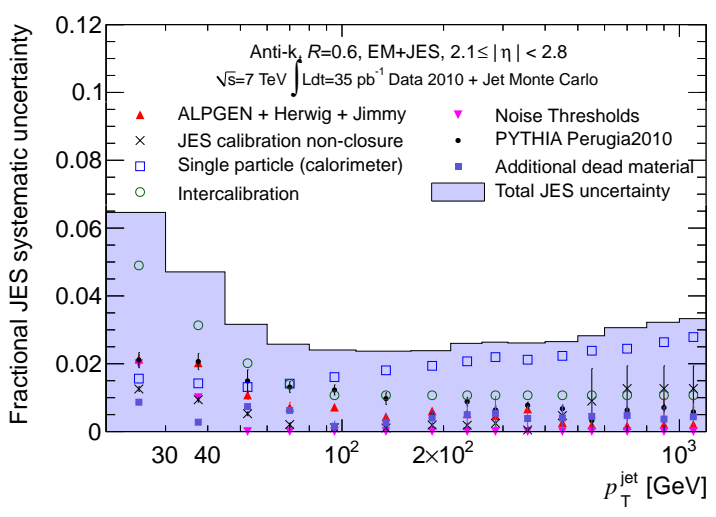

(b)

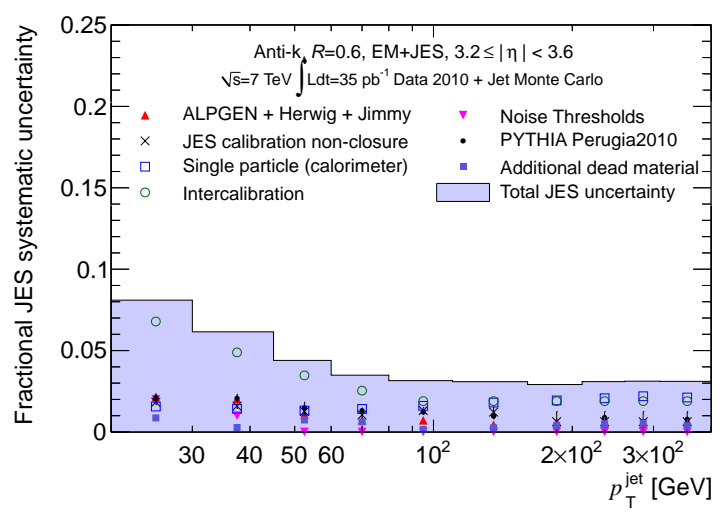

(d)

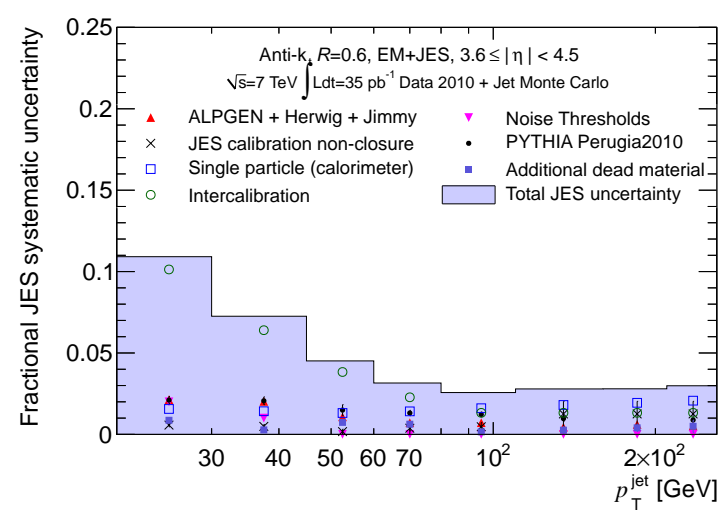

(e)

Figure C.2.:Fractional jet energy scale systematic uncertainty as a function of $p_{\mathrm{T}}^{\text {jet }}$ for jets with distance parameter $\mathrm{R}=0.6$ in the pseudorapidity regions within $1.2 \leq|\eta|<4.5$. The total uncertainty is shown as the solid light blue area. The individual sources are also shown, with uncertainties from the fit if applicable. 


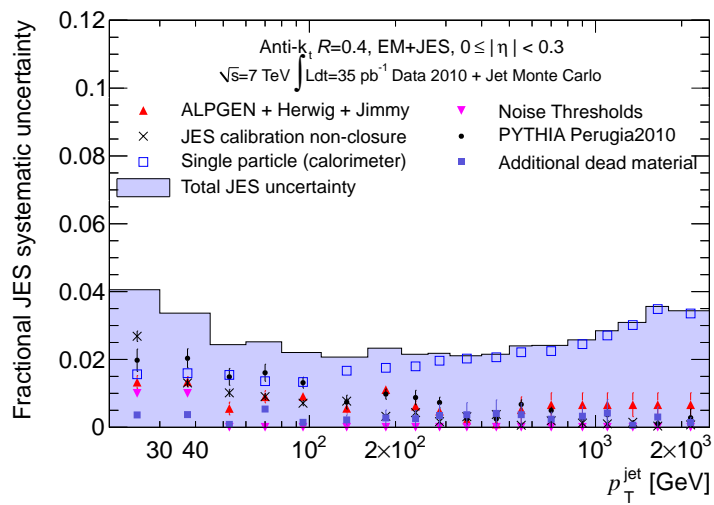

(a)

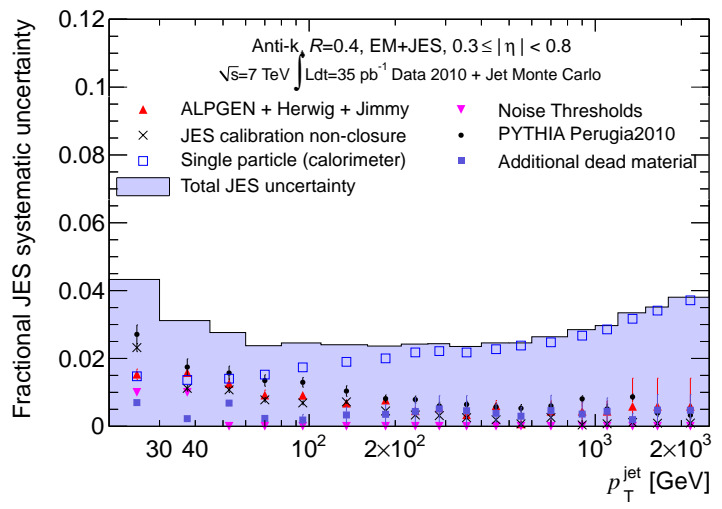

(b)

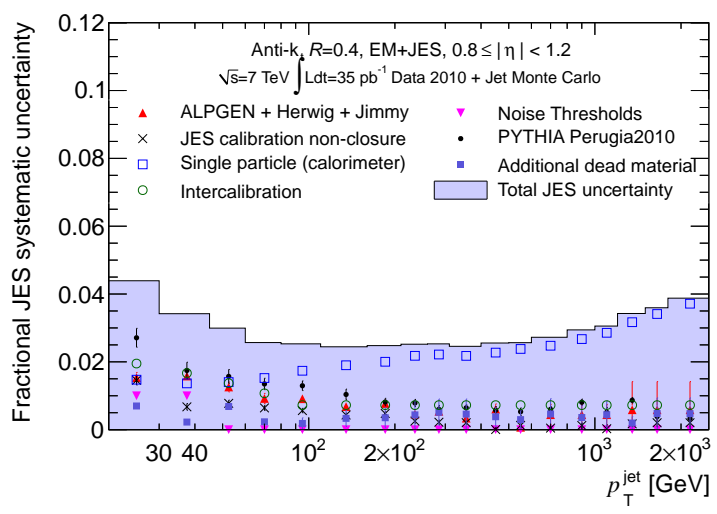

(c)

Figure C.3.:Fractional jet energy scale systematic uncertainty as a function of $p_{\mathrm{T}}^{\text {jet }}$ for jets with distance parameter $\mathrm{R}=0.4$ in the pseudorapidity regions within $0 \leq|\eta|<1.2$. The total uncertainty is shown as the solid light blue area. The individual sources are also shown, with uncertainties from the fit if applicable. 


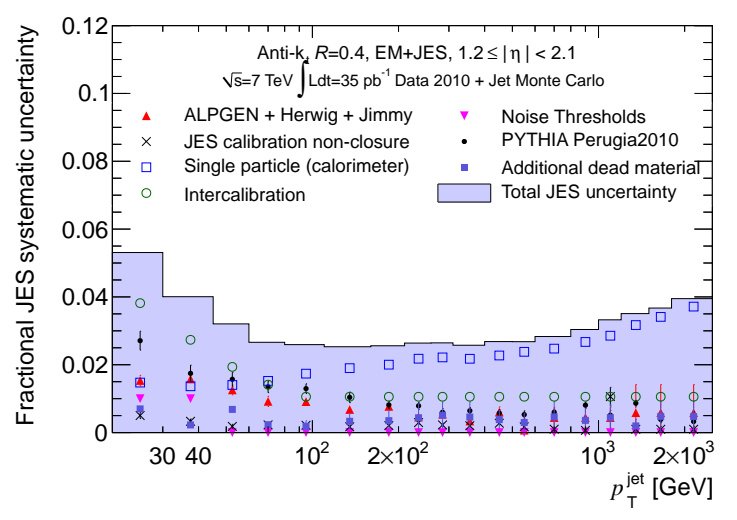

(a)

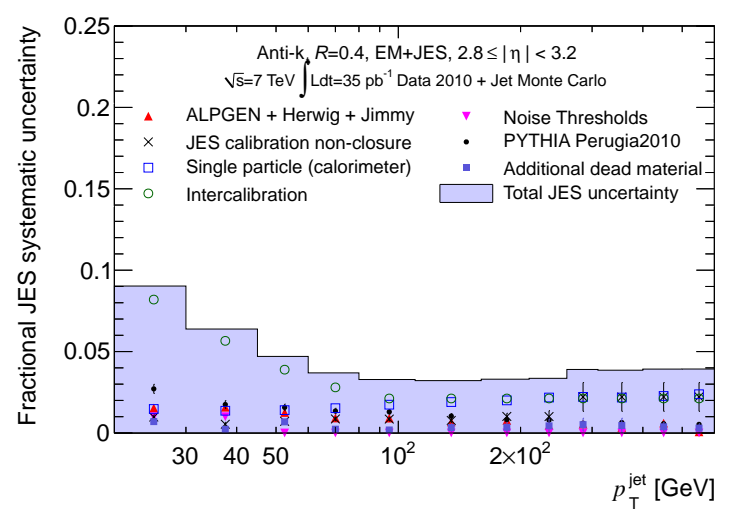

(c)

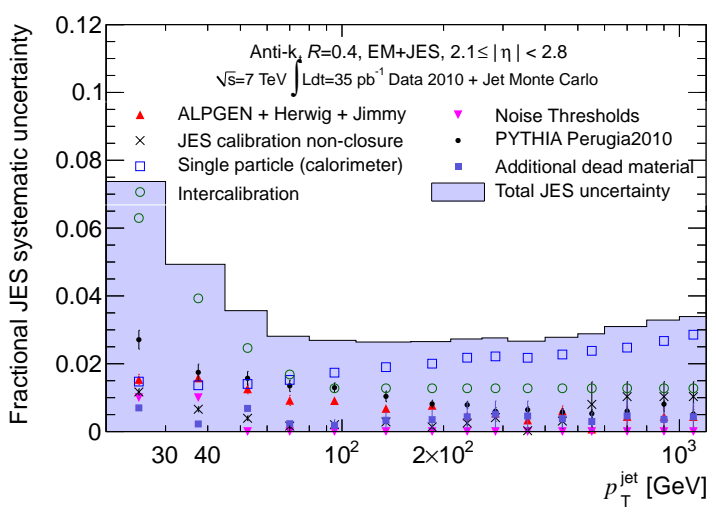

(b)

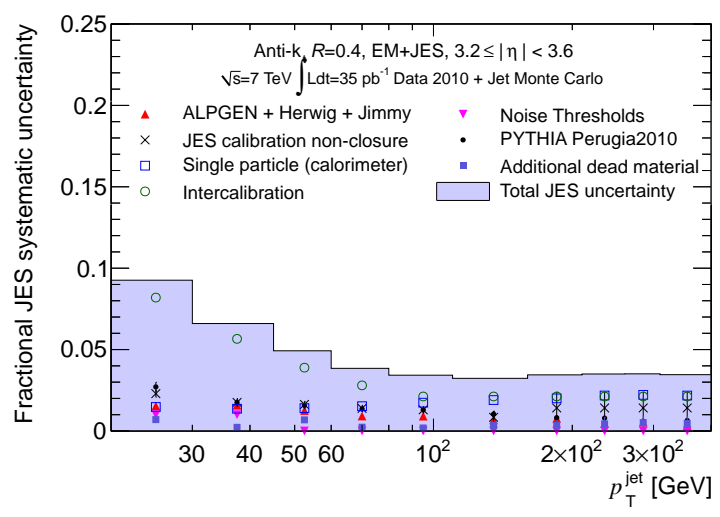

(d)

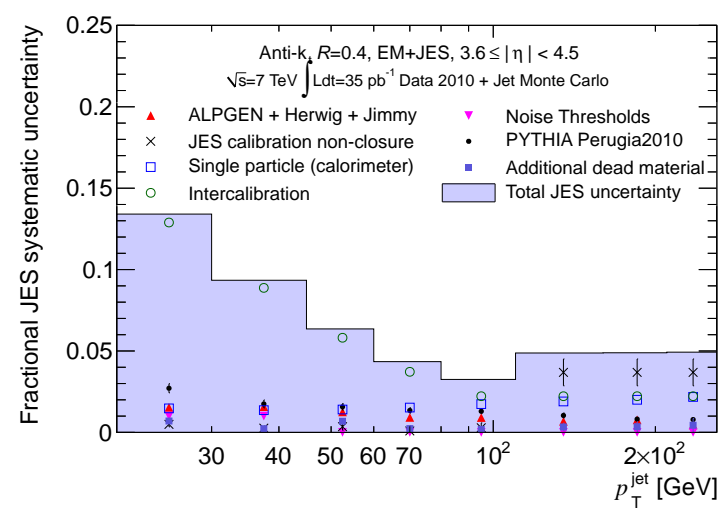

(e)

Figure C.4.:Fractional jet energy scale systematic uncertainty as a function of $p_{\mathrm{T}}^{\text {jet }}$ for jets with distance parameter $\mathrm{R}=0.4$ in the pseudorapidity regions within $1.2 \leq|\eta|<4$.5. The total uncertainty is shown as the solid light blue area. The individual sources are also shown, with uncertainties from the fit if applicable. 


\section{Appendix D.}

\section{First estimate of the JES uncertainty (June 2010)}

This appendix describes the first estimate of the JES uncertainty used in early 2010 analyses (presented at the ICHEP international conference in July 2010), as in Reference [125]. It follows the structure of Chapter 5, and only the relevant differences with respect to the JES uncertainty analysis for the whole 2010 dataset are mentioned.

The first JES uncertainty is largely based on Monte Carlo studies, employing the di-jet balance method only to evaluate the JES uncertainty in the endcap region. The calorimeter contribution to the JES uncertainty is verified with a method that propagates to the jets the uncertainties on the single energy deposits from in-situ and test beam measurements. The energy contribution of multiple proton-proton interactions to calorimeter jets is not accounted for in the JES calibration; it is included as a separate contribution to the systematic uncertainty.

\section{D.1. Analysis details}

The first estimate of the JES uncertainty is provided for jets with transverse momentum greater than $20 \mathrm{GeV}$ and pseudo-rapidity smaller than 2.8, following the binning reported in Table 5.1. The JES systematic uncertainty for all jets with pseudorapidity beyond $\eta=1.2$ in the first estimate is determined using the JES uncertainty for the central barrel region $(0.3<|\eta|<0.8)$ as a baseline, and adding a contribution from the relative calibration of the jets with respect to the central barrel region.

The nominal and systematic Monte Carlo samples used for the analysis of the JES uncertainty have been generated and simulated as described in Section 1.4.2, but use a set of parameters tuned to experimental data taken before the start of the LHC denoted with MC09. These parameters are described in Reference [133].

\section{D.1.1. Jet calibration for the first $17 \mathbf{n b}^{-1}$ of ATLAS data}

In the very first ATLAS data up to Summer 2010 (roughly $17 \mathrm{nb}^{-1}$ ), only the global energy correction step of the calibration described in Chapter 3 is applied. In this case, the jet energy response is determined as a function of jet $p_{\mathrm{T}}^{\text {truth }}$ and $\eta$. Instead of using the average 
reconstructed jet $p_{\mathrm{T}}^{\text {jet,EM }}$ for each $p_{\mathrm{T}}^{\text {truth }}$ bin, an inversion technique [127] is used to transform the dependence of the jet response from a function of the truth jet energy to a function of the reconstructed jet energy at the EM-scale, to ensure that the calibration depends solely on reconstructed quantities and can therefore be applied on data. In the first $17 \mathrm{nb}^{-1}$, the peak number of reconstructed vertices varied between ranges from 0.5 to 2.1, as shown in Figure 2.2, compared to the maximum value of 3-4 for the latter data periods. As described in Section 5.3, the additional energy due to multiple interactions is not been taken into account in the jet energy scale and a pile-up induced systematic uncertainty is provided instead. The ATHENA jet software only retains jets with a calibrated $p_{\mathrm{T}}^{\text {jet }}$ above $7 \mathrm{GeV}$ in the standard data format.

\section{D.1.2. Selection of jets for systematic studies in Monte Carlo studies}

The jet selection for the Monte Carlo studies for the first estimate of the JES uncertainty follows that of Section 5.1.1, but does not include any isolation criteria: the uncertainty had been provided for inclusive QCD jets. Additional studies on jet flavour composition, response and topology that differ with respect to what was used in the derivation of the JES corrections and on the estimate of the JES uncertainty were left to the individual analysis.

\section{D.2. Sources of jet energy scale uncertainty}

Five broad uncertainty categories have been used to characterize the components of the first estimate of the JES uncertainty.

1. the uncertainty due to the JES calibration method is described in Section D.2.1;

2. the uncertainty due to the calorimeter response and to the simulation of the material budget (Section D.2.2);

3. the uncertainty due to the detector simulation (Section D.2.3);

4. the uncertainty due to the physics model and parameters employed in the Monte Carlo event generator (Section D.2.4);

5. the uncertainty due to the relative calibration for jets with $\eta>1.2$ (Section D.2.5).

The relevant differences with respect to the estimates in Section 5.2 are detailed in the following Sections.

\section{D.2.1. Uncertainty due to the JES calibration}

The deviation from unity of the average jet response after the simple EM+JES calibration described in Section D.1.1 in the same sample where the calibration constants were derived is considered as a source of systematic uncertainty. In addition to the motivations reported in Section 5.2.1, the jet selection does not reflect that used to derive the calibration constant due to the absence of a jet isolation cut. The lower response of close-by jets is therefore partly taken into account in the non-closure uncertainty component. 
Figure D.1 shows the jet response for $p_{T}$ and energy as a function of $p_{\mathrm{T}}^{\text {jet }}$ for the nominal Monte Carlo sample in the barrel and endcap regions. The systematic uncertainty due to the non-closure of the nominal JES calibration is taken as the largest deviation of the response from unity between energy and $p_{T}$. In the barrel region $0.3<|\eta|<0.8$ this contribution amounts to about $2 \%$ at low $p_{\mathrm{T}}^{\text {jet }}$ and smaller than $1 \%$ for $p_{\mathrm{T}}^{\text {jet }}>50 \mathrm{GeV}$. In the endcap region, the closure is better than $1 \%$ for $p_{\mathrm{T}}^{\text {jet }}>20 \mathrm{GeV}$, while the energy response is within $1 \%$ for jets with transverse momentum above $30 \mathrm{GeV}$.

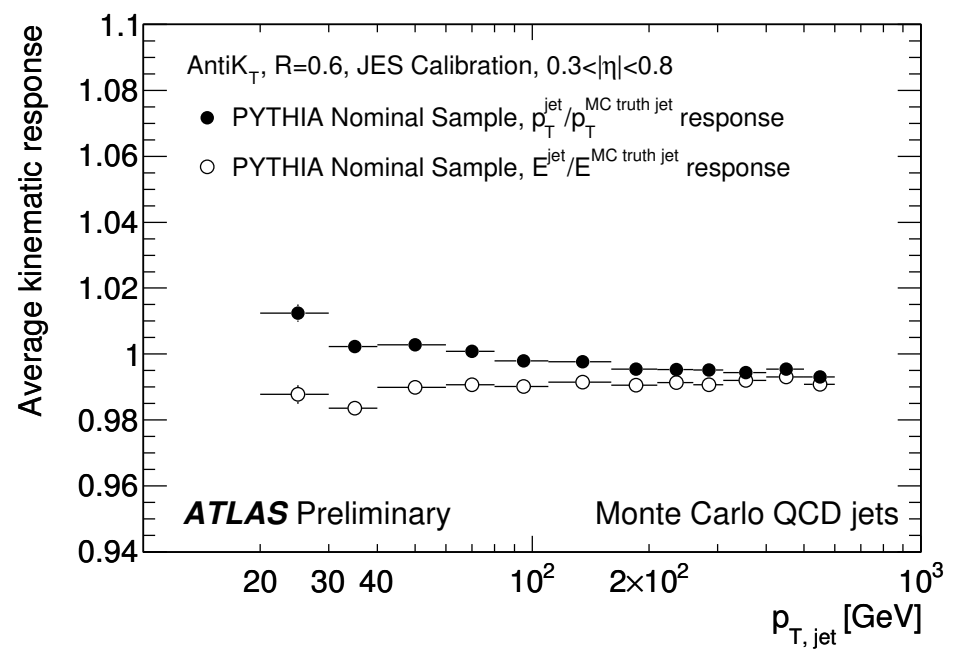

(a)

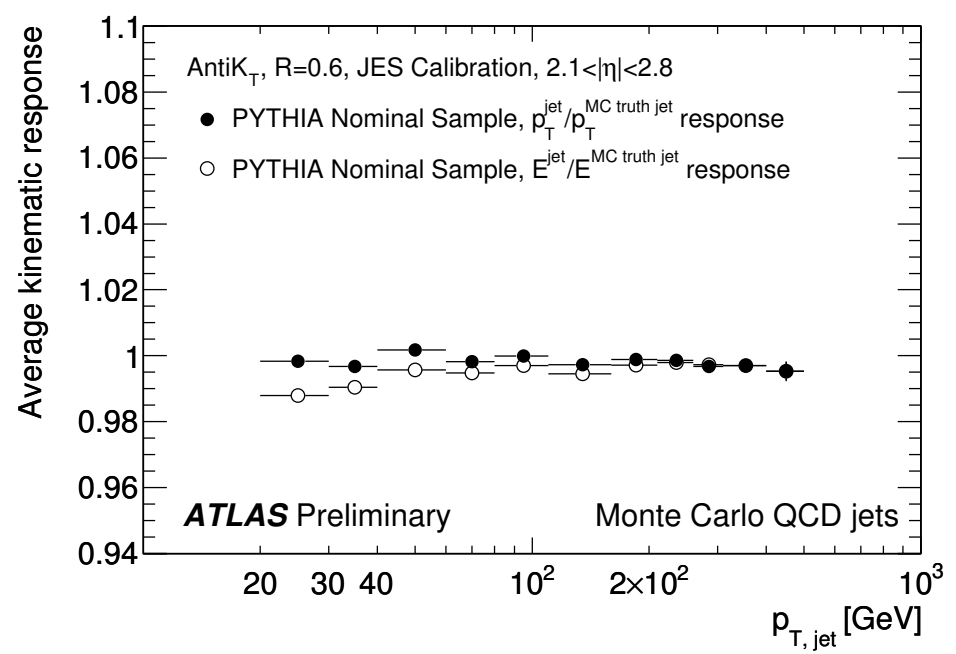

(b)

Figure D.1.:Closure test for JES calibration: simulated jet response for the nominal sample of inclusive PYTHIA jets after the JES calibration. $p_{\mathrm{T}}^{\text {jet }}$ (solid circles) and energy (open circles) response as a function of $p_{\mathrm{T}}^{\text {jet }}$ in the central barrel (top) and endcap (bottom) regions. 
The non-closure uncertainty was conservatively considered fully correlated to the other components in the first JES uncertainty analysis and added linearly to the other uncertainty components.

\section{D.2.2. Calorimeter response and dead material uncertainty}

In the first JES uncertainty analysis, the uncertainty on the calorimeter response was derived using Monte Carlo simulation samples. The calorimeter response uncertainty estimated using the single particle analysis was available, but it was only used as a cross-check.

The uncertainties on the single particle response used to cross-check the calorimeter component for the first JES analysis differ from those detailed in Section 5.2.2 in the following:

- The uncertainty on the response of neutral hadrons was conservatively estimated to amount to an additional $20 \%$ uncertainty on top of the uncertainty for charged particles is considered.

- The absolute electromagnetic scale of the Liquid Argon calorimeter does not benefit by any in-situ calibration, and it amounts to 3\% [77]. Similarly, the first analysis of the Tile electromagnetic scale lead to an uncertainty of $4 \%$ [81].

The uncertainty sources related to the calorimeter response used in the first JES uncertainty analysis are:

Uncertainties due to the hadronic shower model:The properties of the hadronic showering of particles interacting in the calorimeter influence the shape and extent of the energy deposits and therefore the jet energy scale. The contributions to the JES uncertainty from the hadronic shower model are evaluated using the following Monte Carlo samples:

- QGSP the QGSP set of parameters for hadronic interactions in the detector simulated by GEANT4 follows the QGSP_BERT details described in Section 2.6 and used in the nominal simulation sample, but with the Bertini nucleon cascades not simulated.

- FTFP_BERT the Quark Gluon String fragmentation model from the QGSP_BERT is substituted with the Fritiof model [183]. In this model, the low energy treatment of quasi-elastic nucleon scattering and nuclear diffraction process is improved at low eneergies with respect to the Quark Gluon String model.

ATLAS test-beam data for single pions with energies ranging from 2 to $180 \mathrm{GeV}$ have been compared to simulations using the two set of parameters for the description of hadronic showers above. In this study, described in [91], the measured mean single pion response has been shown to lie within these two descriptions. Below $2 \mathrm{GeV}$, QGSP_BERT and FTFP_BERT follow the Bertini cascades model, while the QGSP follows the Low Energy Parameterized (LEP). Monte Carlo simulations of single particles that make use of the QGSP BERT model and analysis of isolated tracks matched to energy deposits [49] agree within 5\%, while the LEP description has a much lower response with respect to the Bertini model at low energies [87]. Therefore, the choice of these two simulation samples provides a safe range for the uncertainty due to the hadronic showering model over the whole $p_{T}$ range of particles in jets. 
The Monte Carlo simulation confirms the expectations from the test beam results: the FTFP_BERT description predicts hadronic showers in the detector that are longer and narrower than in the data, whereas the absence of Bertini cascades make the showers shorter. The effect of the different hadronic shower models on the jet response is shown in figure D.2. A $4 \%$ lower jet response is observed in the central (barrel) region when the nominal calibration, derived using the QGSP BERT hadronic shower model, is applied to the QGSP hadronic shower model sample. This difference is approximately independent of $p_{\mathrm{T}}^{\text {jet }}$. In the FTFP_BERT shower model sample, the jet response is between $2 \%$ and $4 \%$ higher than unity.

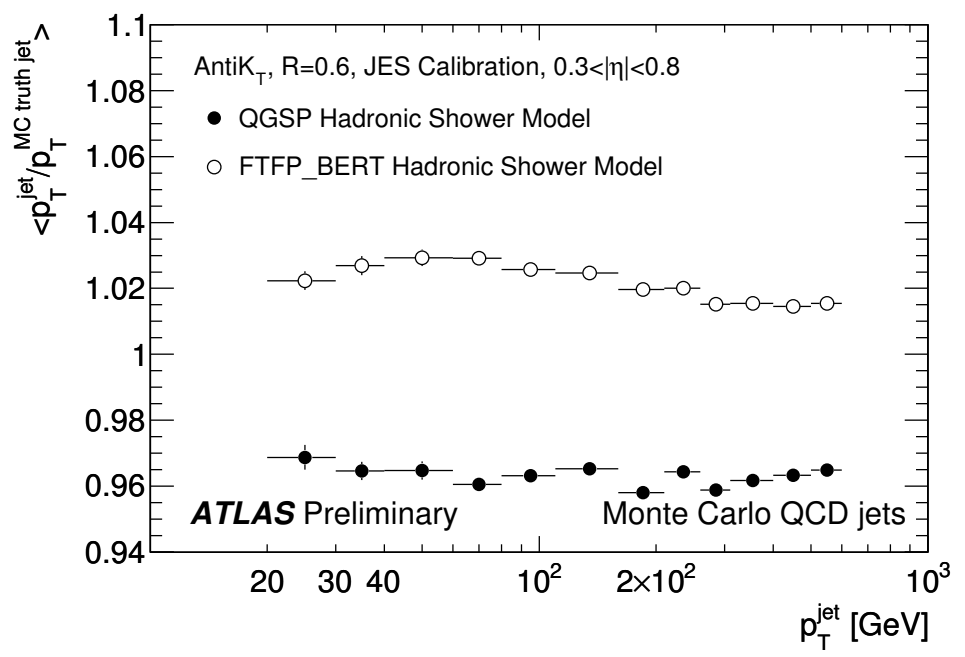

Figure D.2.:Simulated jet $p_{T}$ response after the JES calibration as a function of $p_{\mathrm{T}}^{\text {jet }}$ for the QGSP (open circles) and FTFP_BERT (solid circles) hadronic shower models in the central barrel region.

\section{Uncertainties on the material budget in the simulation:}

In the case of the first JES uncertainty estimate, no in-situ measurement that could constrain the material budget uncertainty was used. Therefore, the contribution from additional inactive material was entirely derived from Monte Carlo studies.

The distorted detector geometry used for the first JES uncertainty estimated includes:

- an extra 0.05 radiation length $\left(X_{0}\right)$ has been placed radially between the barrel pre-sampler and the calorimeter itself $(|\eta|<1.45)$;

- an extra $0.2 X_{0}$ of dead material has been placed radially in the cryostat before the electromagnetic calorimeter $(|\eta|<1.5)$, motivated by the largest possible change in the material budget in test-beam measurements [73];

- an extra $0.2 X_{0}$ has been placed radially in the cryostat between the barrel of the electromagnetic calorimeter and the hadronic calorimeter, motivated by the comparison of $900 \mathrm{GeV}$ data to Monte Carlo; 
- the density of the material of the barrel-endcap cryostat gap (covering the space between electromagnetic barrel and electromagnetic/hadronic endcaps for $1<|\eta|<1.4$ and in front of the electromagnetic endcap up to $|\eta|<1.8$ ) has been increased by a factor 1.5.

The uncertainty on the jet energy scale due to the the dead material description for the first iteration of the JES uncertainty is shown in Figure D.4. The response in the dead material sample shows a $2 \%$ downward shift on the jet response, almost independent of $p_{\mathrm{T}}^{\text {jet }}$.

Uncertainties on the energy scale of the ATLAS calorimeter: As mentioned above, the uncertainties on the absolute electromagnetic scale of the Liquid Argon and Tile calorimeters amount respectively to $3 \%$ and $4 \%$, flat in $\eta$ and $p_{\mathrm{T}}^{\text {jet }}$. The Liquid Argon uncertainty is scaled by the average jet energy deposited in the electromagnetic calorimeter as a function of $p_{\mathrm{T}}^{\text {jet }}$, while the Tile uncertainty is scaled by the jet energy deposited in the hadronic calorimeter. The two uncertainties are finally combined to form the uncertainty on the absolute electromagnetic energy scale.

\section{Comparison of calorimeter uncertainty used in the first JES uncertainty estimate and single particle calorimeter uncertainties}

In the central calorimeter region $(0<|\eta|<0.3$ and $0.3<|\eta|<0.8)$, the convolution of the response and uncertainty of single particles within a jet [49] can be used to obtain a cross check for the Monte Carlo based estimate of the JES uncertainty contributions from the calorimeter measurement.

The contributions to the calorimeter uncertainty used in the first JES uncertainty estimate are combined as follows:

1. For each $p_{\mathrm{T}}^{\text {jet }}$ bin in the pseudorapidity bin considered, the ratio between the response in the Monte Carlo test sample with a systematic variation $R_{\text {var }}$ and the response in the nominal sample $R_{\text {nom }}$ is considered as a starting point for the estimate of the JES uncertainty. The deviation of this ratio from unity is defined as $\Delta_{\mathrm{JES}}\left(p_{T}^{\text {jet }}, \eta\right)$. This maximum deviation between the energy and $p_{T}$ response is retained for each bin.

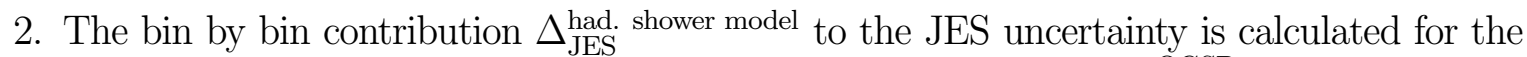
individual deviations for the two different hadronic shower models $\left(\Delta_{\mathrm{JES}}^{\mathrm{QGSP}}\right.$ and $\left.\Delta_{\mathrm{JES}}^{\mathrm{FTFP}} \mathrm{BERT}\right)$ as:

$$
\Delta_{\mathrm{JES}}^{\text {had. shower model }}\left(p_{T}^{\text {jet }}, \eta\right)=\max \left(\Delta_{\mathrm{JES}}^{\mathrm{QGSP}}\left(p_{T}^{\text {jet }}, \eta\right), \Delta_{\mathrm{JES}}^{\mathrm{FTFP} B E R T}\left(p_{T}^{\text {jet }}, \eta\right)\right)
$$

3. The uncertainty due to additional material upstream the calorimeters is estimated using the jet response in dedicated samples described in 5.2.3 in a way similar to the hadronic shower model contribution. 
4. The absolute electromagnetic energy scale contribution from the electromagnetic (3\%) and hadronic calorimeter $(4 \%)$ is weighted by the average electromagnetic and hadronic energy content for jets in a given $p_{T}, \eta$ bin.

5. The three components mentioned above are added in quadrature to obtain the Monte Carlo based estimate of the calorimeter response uncertainty used in the first JES uncertainty estimate.

As explained in Section 5.2.3, single particle studies do not fully account for the uncertainty due to additional material before the calorimeters. To allow a fair comparison between the single particle and the Monte Carlo based estimates, the contribution of the Monte Carlo additional material uncertainty is scaled by the simulated particle content of the jet in electrons, photons and charged hadrons in the combined test beam and added to the estimate of the JES uncertainty from single particle studies.

The calorimeter JES uncertainty derived from single particle studies is compared to the JES uncertainty related to the calorimeter measurement derived from Monte Carlo studies in the central region (up to $|\eta|<0.8$ ) in Figure D.3.

The estimate of the calorimeter-related JES uncertainty from Monte Carlo studies is 2-3\% higher with respect to the single particle studies. This can be explained by the conservative choices made in the parameters of the GEANT4 simulation used for the estimate of the hadronic shower uncertainty in the Monte Carlo-based study. Furthermore, the uncertainty on the energy deposits for particles below $20 \mathrm{GeV}$ is estimated through $\mathrm{E} / \mathrm{p}$ studies with $\sqrt{s}=7 \mathrm{TeV}$ data in the ATLAS detector: the uncertainty in this case is constrained by the in-situ study and the overall uncertainty on jets composed by these particles is reduced with respect to the Monte Carlo study.

\section{D.2.3. Uncertainties due to the detector simulation}

\section{Calorimeter cell noise thresholds}

In order to estimate the uncertainty due to discrepancies in the noise modelling between the data and in the simulation that could affect jet reconstruction through the topocluster noise thresholds, all topocluster thresholds in the simulation are shifted upwards and downwards during jet reconstruction. A Monte Carlo sample has been reconstructed with signal-to-noise thresholds for all topocluster seed and neighbor cells modified to be $10 \%$ higher and lower than the respective nominal values of 4 and 2. Seed thresholds of 4.4 and 3.6 and neighbors thresholds of 2.2 and 1.8 have been used. The $10 \%$ variation provides a conservative estimate of the uncertainty on the noise description that takes into account the stability of the noise spread in dedicated noise runs and the comparison of the noise distribution in data and Monte Carlo.

As shown in Figure D.4, the uncertainty from topoclusters thresholds contributed to the largest and most non-linear contribution to the JES uncertainty, up to $4 \%$ at low $p_{\mathrm{T}}^{\text {jet }}$. In the case of lowering the topocluster thresholds, the topoclusters will include more cells containing a prevalence of noise; at low $p_{\mathrm{T}}^{\text {jet }}$ jets are composed by a larger number of topoclusters so the 


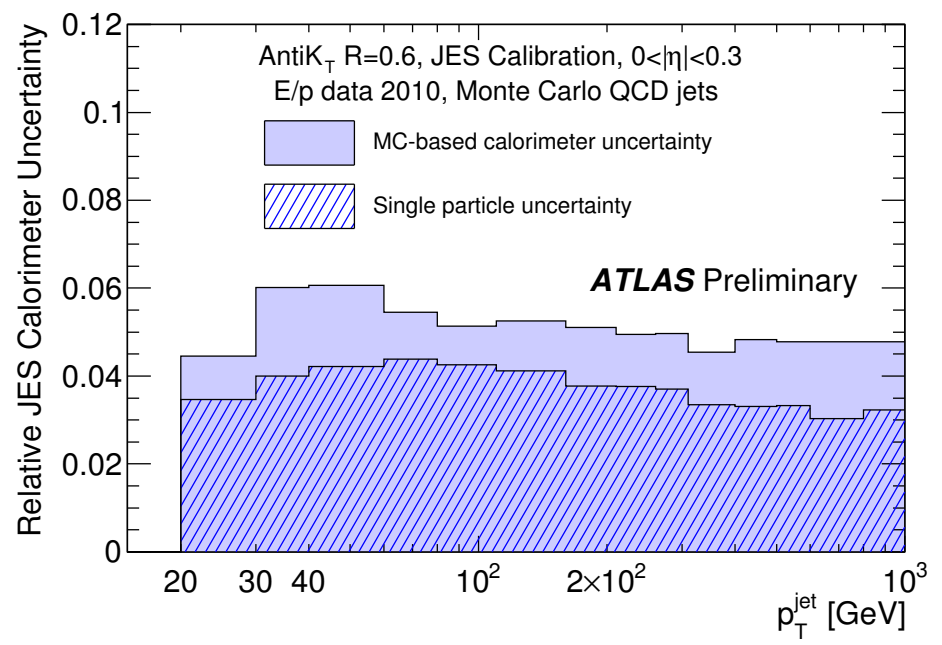

(a)

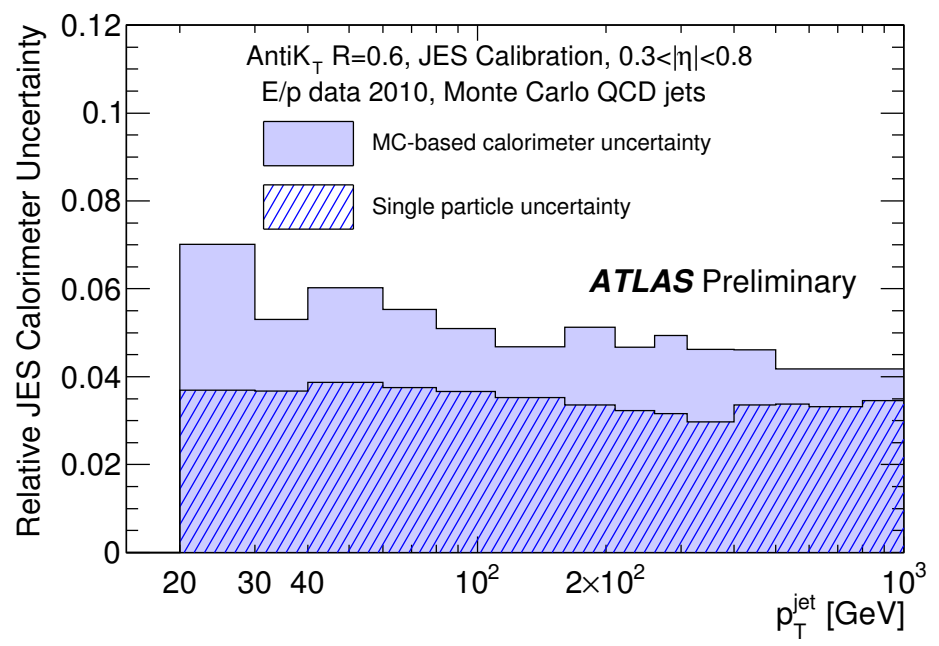

(b)

Figure D.3.:Relative jet energy scale systematic uncertainty due to the calorimeter measurement as a function of jet $p_{T}$ for central jets, estimated from single particle analysis (hatched) and from Monte Carlo studies (solid).

effect will be more prominent. Therefore in this region jets are more energetic with respect to the nominal Monte Carlo sample which was used to derive the JES correction constants.

\section{Beam spot}

In the first 2010 data reprocessing, the calibration scheme did not include the correction to the jet origin mentioned in Section 3.2.1. The jet reconstruction therefore uses $(x, y, z)=(0,0,0)$ (the centre of the detector and origin of the ATLAS coordinate system) as reference to calculate the direction and $p_{T}$ of the input jet constituents. If the beam spot is shifted with respect to the detector center, the origin of the jet could differ from the one assumed by the reconstruction, 
and the jet $p_{T}$ could be biased as a consequence. Monte Carlo truth jets are not affected by this, since the origin of their four-vectors are the simulated vertices. The jet energy scale could still be affected by differences between the beam spot position with respect to the detector centre between data and the nominal MC sample ${ }^{1}$, and this is taken into account as an additional source of systematic uncertainty. The variation on the jet energy scale from differences in the beam spot position between data and Monte Carlo simulation is evaluated using a sample generated with a shifted beam-spot based on early LHC parameter estimates of $(x, y, z)=(1.5,2.5,-9)$ $\mathrm{mm}$. These parameters cover the shift in the current average coordinates observed from data collected by ATLAS from LHC collisions: $(x, y, z)=(-0.4,0.62,-1.3) \mathrm{mm}$.

Figure D. 4 shows the individual effects of the shifted beam spot position on the jet $p_{\mathrm{T}}^{\text {jet }}$ response for the first estimate of the JES uncertainty, together with the effects of the dead material description in the simulation, and the topocluster thresholds. The shifted beam spot mostly affects the jet response at low $p_{\mathrm{T}}^{\text {jet }}$. For $p_{\mathrm{T}}^{\text {jet }}>30 \mathrm{GeV}$, the beam spot variation only contributes less than $1 \%$ to the overall JES uncertainty.

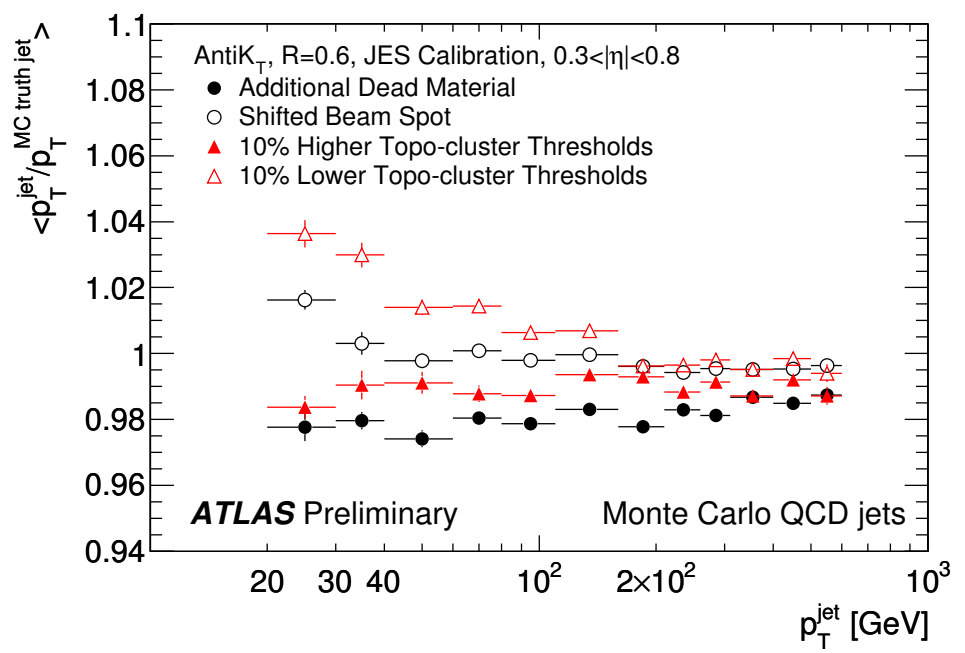

Figure D.4.:Simulated jet $p_{\mathrm{T}}^{\text {jet }}$ response after the JES calibration as a function of $p_{\mathrm{T}}^{\text {jet }}$ for samples with varied dead material (solid circles), beam spot position (open circles), and topocluster noise thresholds varied by $10 \%$ (triangles) in the central barrel (top) and endcap (bottom) regions.

\section{D.2.4. Uncertainties due to the event modelling in the Monte Carlo generators}

The contributions to the JES uncertainty from the modelling of the fragmentation and underlying event and other parameters of the Monte Carlo event generator are obtained using the following Monte Carlo samples:

- Alpgen + Herwig + Jimmy: the combination of the ALPGEN generator interfaced to HeRWIG and JimmY is used to test the effects of a different modelling of the hard

\footnotetext{
${ }^{1}$ The position of the average beam spot for the nominal MC sample is $(x, y, z)=(-0.15,1,0) \mathrm{mm}$.
} 
subprocess and soft processes. In the case of the JES uncertainty for the full 2010 dataset, this configuration is tuned to pre-LHC collider data in a manner similar to the Pythia MC09 tune.

- Pythia MC09 tune with Perugia fragmentation: this set of parameters for the PyTHIA generator uses a different underlying-event model with respect to the nominal sample. In this tune, the soft-QCD part is tuned using only minimum-bias data from the Tevatron and CERN $p \bar{p}$ colliders [184].

- Pythia MC09 tune with Professor fragmentation: the set of parameters used for the PYTHIAgenerator has been tuned to LEP data with the Professor software [185] and differs from the nominal ATLAS MC09 tune in the shape and parameters of the fragmentation function.

The effect on the jet response from the different sources of theoretical uncertainties is shown in Figure D.5. The maximum deviation of the response from unity is observed for $E^{\text {jet }} / E^{\mathrm{MC}}$ particle jet in the ALPGEN sample with a downward shift of about $4 \%$ for $p_{T}<50 \mathrm{GeV}$.

\section{D.2.5. Endcap uncertainty due to relative calibration}

In the first iteration of the JES uncertainty, the strategy used to estimate the JES uncertainty in the endcap region (starting from $|\eta|>1.2$ since dead material samples were available up to that pseudorapidity) was to consider two sources of uncorrelated JES systematic uncertainties from the eta intercalibration measurement from transverse momentum balance (Section 4.2.1):

- the difference between data and Monte Carlo in the relative energy scale (up to $2.4 \%$ for jets in $2.1<|\eta|<2.8)$.

- the deviation from one of the relative energy scale in the data (up to $2 \%$ in $2.1<|\eta|<2.8$ ).

The relative $\eta$ inter-calibration uncertainty is determined for jets where the average $p_{\mathrm{T}}^{\text {jet }}\left(p_{T}^{\text {avg }}\right)$ of the two leading jets is between $50 \mathrm{GeV}$ and $110 \mathrm{GeV}$ and the resulting uncertainty is applied to all $p_{\mathrm{T}}^{\text {jet }}$. This choice is made on the basis that this uncertainty includes jets from the lower $p_{\mathrm{T}}^{\text {jet }}$ considered in this study; for higher $p_{\mathrm{T}}^{\text {jet }}$ than what is considered in the intercalibration study the main sources of uncertainties had been shown to decrease. The dead material uncertainty is also expected to decrease with increasing energy. Therefore this choice leads to a conservative estimate of the uncertainty in the endcap region.

Figure D.6 shows the relative jet response measured in both data and simulation as a function of jet $|\eta|$ used for the first estimate of the JES uncertainty.

\section{D.3. Uncertainty due to multiple interactions}

Over the course of the first data taking period up to July 2010, the LHC beam intensity has been increased and the size of the interaction region reduced in order to increase the LHC luminosity. Therefore, even the very first LHC data is affected by in-time pile-up, albeit to a reduced extent with respect to further data taking periods. 


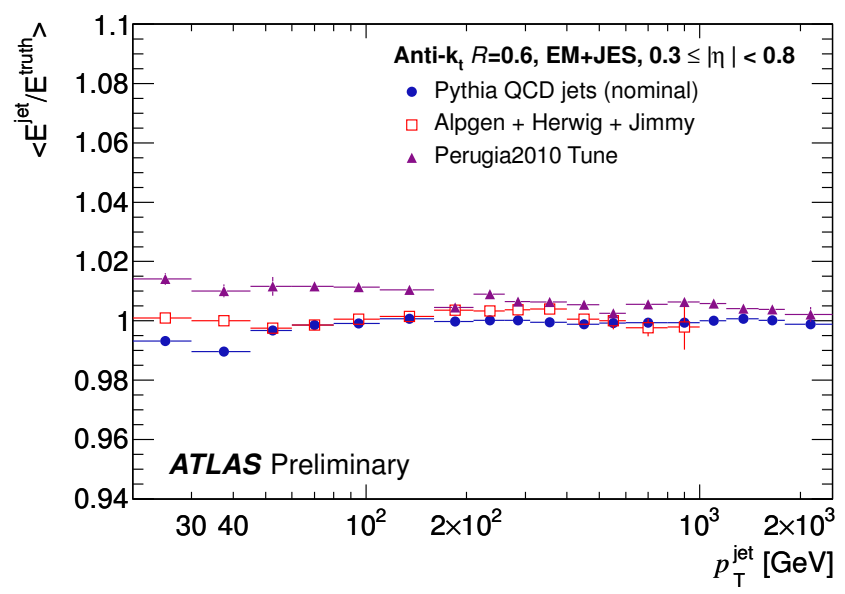

(a)

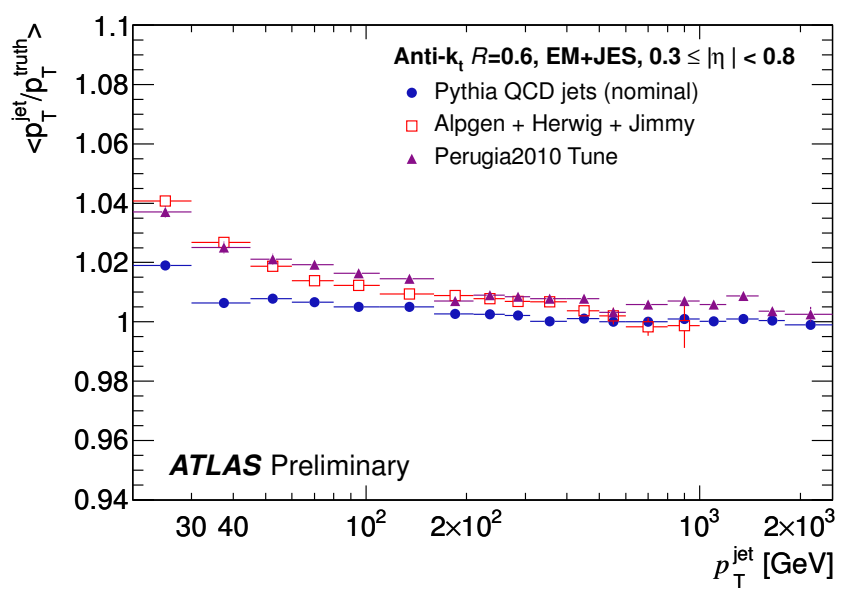

(b)

Figure D.5.:Simulated jet $p_{\mathrm{T}}^{\text {jet }}$ and energy response as a function of $p_{\mathrm{T}}^{\text {jet }}$ in the central region $(0.3<|\eta| \leq 0.8)$ for AlPGEN + Herwig + Jimmy (full triangles), Pythia with the Perugia0 tune (full circles), and Pythia with the Professor fragmentation model (open circles).

The offset correction described in Section 3.2.1 was not applied, but instead used as the basis for estimating an uncertainty due to the additional energy from pile-up interactions affecting the jet response.

The average impact of pile-up is considered as an additional source of systematic uncertainty and it is estimated directly from data, assuming that all jets and events are affected equally regardless of the number of primary vertices ${ }^{2}$. This provides an upper bound on the uncertainty due to pile-up effects for this early data taking period, and is added to the baseline jet energy scale uncertainty.

\footnotetext{
${ }^{2}$ This is motivated by the average fraction of events having only one additional primary vertex in the data- set
} considered. 


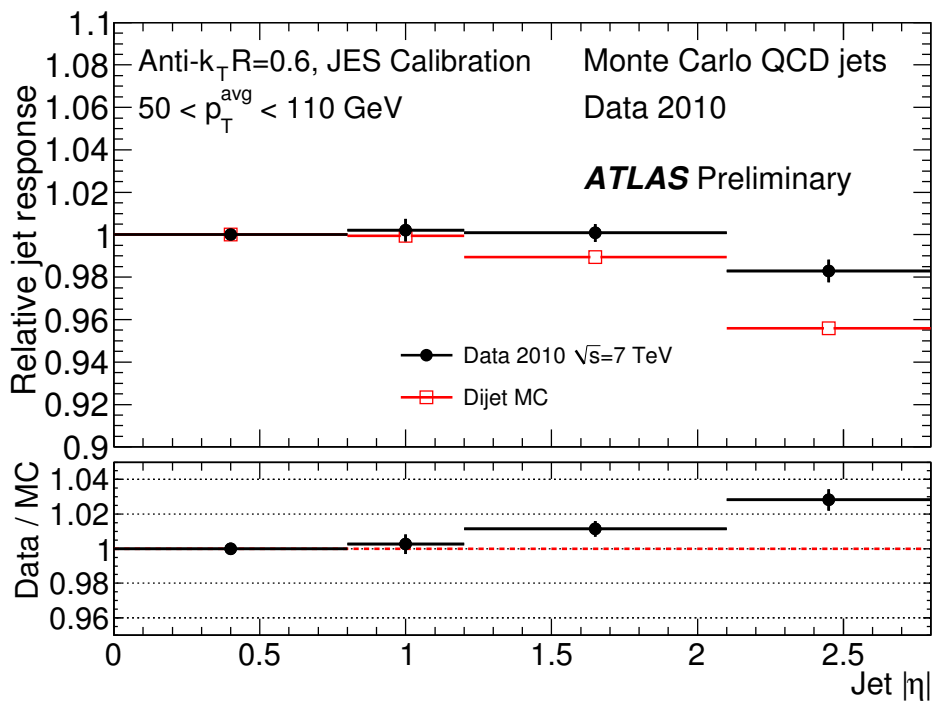

Figure D.6.:Jet $p_{\mathrm{T}}^{\text {jet }}$ response $\left(p_{T}^{\text {jet,probe }} / p_{T}^{\text {jet,reference }}\right)$ after the JES calibration relative to jets in the central region $(0<|\eta|<0.8)$ used as a reference, obtained by exploiting the $p_{\mathrm{T}}^{\text {jet }}$ balance of as a function of jet $\eta$ in data and simulation.

For each run, the extra transverse energy contributing to a jet is estimated from the energy deposited in the calorimeter towers as:

$$
\Delta p_{T}^{\text {jet }}\left(\eta,\left\langle N^{\text {pile-up }}\right\rangle\right)=\frac{\Delta E_{T}(\eta)}{A(\eta) \cdot N_{v t x}} \cdot A(\eta) \cdot\left\langle N^{\text {pile-up }}\right\rangle
$$

In this formula, the additional energy $\left(\Delta E_{T}(\eta)\right)$ per unit of area $(A)$ and average number of interactions $\left(N_{v t x}\right)$ is denoted as $\frac{\Delta E_{T}}{A \cdot N_{v t x}}(\eta)$ and it is evaluated counting the number of reconstructed vertices $N_{v t x}$. It is measured from events selected by the calorimeter trigger as follows:

- For events with $N$ additional interactions the mean tower energy is measured as a function of $\eta$.

- The additional energy per tower and number of additional interactions is determined by subtracting out the average tower energy for events with exactly one interaction from the average tower energy for events with $N$ additional interactions; for example, for a 2-vertex event:

$$
\Delta E_{T}=E_{T}\left(N_{P V}=2\right)-E_{T}\left(N_{P V}=1\right)
$$

This subtraction removes the contribution from the underlying event, which is not part of the pile-up systematic uncertainty. Approximately the same $\Delta E_{T}$ is observed for $E_{T}\left(N_{P V}=\right.$ $3)-E_{T}\left(N_{P V}=2\right)$. 
$A(\eta)$ is the average number of towers in jets $\left(\left\langle N_{\text {tower }}\right\rangle\right)$, as a function of jet $\eta$, and $\left\langle N^{\text {pile-up }}\right\rangle$ is the average number of additional interactions, conservatively estimated as the mean value plus one standard deviation of the distribution of the number of reconstructed additional primary vertices $\left(N=N_{v t x}-1\right)$ :

$$
\left\langle N^{\text {pile-up }}\right\rangle=[\langle N\rangle+R M S(N)] .
$$

The relative JES uncertainty is then obtained by a luminosity-weighted average of $\Delta p_{T}^{\text {jet }} / p_{T}^{\text {jet }}$ in each $\eta$ region. Figure D.7 shows the estimated JES systematic uncertainty contribution from pile-up as a function of $p_{\mathrm{T}}^{\text {jet }}$ and for several $\eta$ regions.

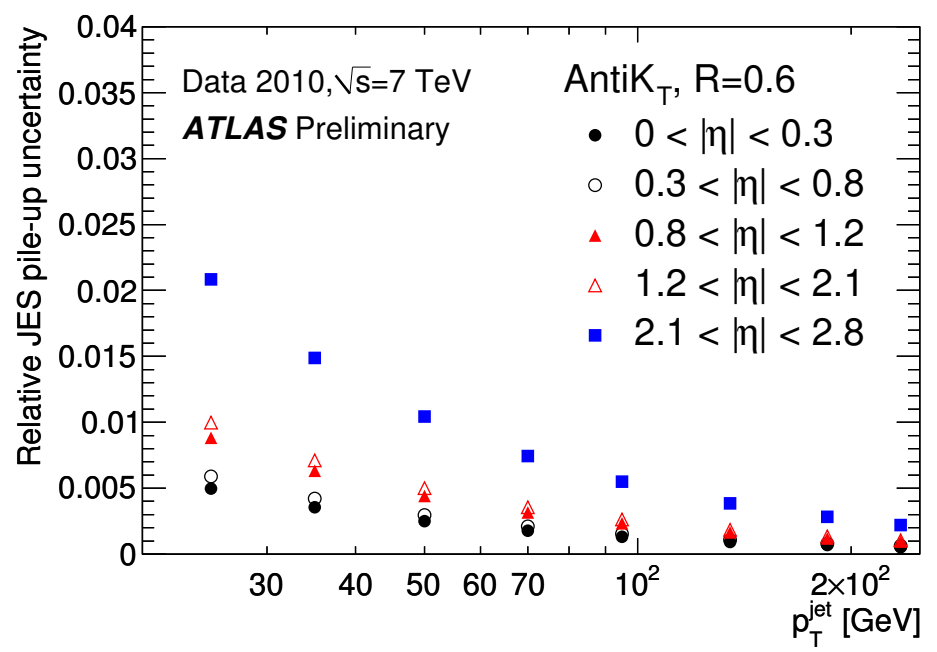

Figure D.7.:Relative JES systematic uncertainty from pile-up as a function of $p_{\mathrm{T}}^{\text {jet }}$ in various $\eta$ regions, estimated as an average from all ATLAS runs up to May 17th, 2010.

The estimate of the pile-up relative systematic uncertainty using this method considers all ATLAS runs up to May 17th, 2010, and corresponds to $7 \mathrm{nb}^{-1}$ of integrated luminosity. For jets with $20<p_{T}^{\text {jet }}<50 \mathrm{GeV}$, the pile-up relative systematic uncertainty is about $1 \%$ in the barrel and $1-2 \%$ in the endcaps. For $p_{\mathrm{T}}^{\text {jet }}>50 \mathrm{GeV}$, the pile-up uncertainty is only significant for $|\eta|>2.1$ and it is smaller than $1 \%$.

\section{D.4. Combination of JES uncertainty sources}

The combination of the contributions described in section D.2 to form the first estimate of the JES uncertainty follows the method outlined in Section 5.4. The two differences with respect to that method are:

- Since th contributions to the JES uncertainty due to increasing or lowering the topocluster noise thresholds are not fully independent, the largest deviation from either of the two effects in each $p_{\mathrm{T}}^{\text {jet }}$ bin is taken as the source of uncertainty. This is also the case for the 
uncertainty due to the two hadronic shower models. Taking these as an example, the bin

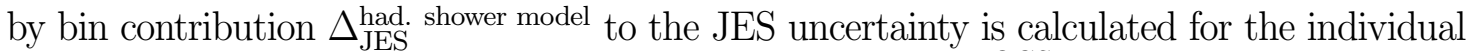
deviations for the two different hadronic shower models $\left(\Delta_{\text {JES }}^{\text {QGSP }}\right.$ and $\left.\Delta_{\text {JES }}^{\text {FTFP_BERT }}\right)$ as in Equation 2.

- The contribution from the deviation from unity of the JES closure test described in section D.2.3 is conservatively considered fully correlated and added linearly.

- The absolute electromagnetic energy scale contribution from the electromagnetic and hadronic calorimeter is weighted by the average electromagnetic and hadronic energy content for jets in a given $p_{T}, \eta$ bin and added in quadrature to the other $\Delta_{J E S}\left(p_{T}^{\text {jet }}, \eta\right)$ contributions.

- The $\eta$ intercalibration contribution is estimated for each pseudorapidity bin in the endcap region from the plot in figure D.6. It is considered flat over the whole $p_{\mathrm{T}}^{\text {jet }}$ range, and added in quadrature to the total JES uncertainty determined in the $0.3 \leq|\eta| \leq 0.8$ region to estimate the JES uncertainty for jets with $|\eta| \geq 1.2$.

- At large $p_{\mathrm{T}}^{\text {jet }}$, the number of events in the Monte Carlo samples becomes insufficient for an estimate of the uncertainty. The uncertainty from the last $p_{\mathrm{T}}^{\text {jet }}$ bin is then used, on the grounds that at higher $p_{\mathrm{T}}^{\text {jet }}$ the largest systematics (noise description, dead material) will be reduced since the measured calorimeter energy is larger.

\section{D.5. Results}

Figures D.8 and D.9 show the final relative jet energy scale systematic uncertainty as a function of jet $p_{\mathrm{T}}^{\text {jet }}$ for the central an endcap regions in the case of no pile-up. Both the total uncertainty (light blue area) and the individual contributions are shown, with statistical errors from the fitting procedure if applicable. On the right hand side of the figures the plots for the final JES uncertainty of Section 5.5 are shown, drawn using the same scale to show the improvement achieved with the new estimate.

The relative JES uncertainty in the central region amounts to approximately $9.4 \%$ for jet $p_{\mathrm{T}}^{\text {jet }}<60 \mathrm{GeV}$, and $7 \%$ for $p_{\mathrm{T}}^{\text {jet }}>60 \mathrm{GeV}$. The uncertainty is increased to up to $10 \%$ and $7.6 \%$ respectively for $p_{\mathrm{T}}^{\text {jet }}<60 \mathrm{GeV}$ and $p_{\mathrm{T}}^{\text {jet }}>60 \mathrm{GeV}$ in the endcap region, where the central uncertainty is taken as a baseline and the uncertainty due to the relative calibration is added.

The dominant contributions to the uncertainty are those due to the detector geometry, the noise description, and the hadronic shower model. Table D.1 presents a summary of the maximum uncertainties in the different $\eta$ regions for anti- $k_{t}$ jets with distance parameter of 0.6 and with $p_{\mathrm{T}}^{\text {jet }}$ above $20 \mathrm{GeV}$ and $60 \mathrm{GeV}$.

The same study has been repeated for anti- $k_{t}$ jets with distance parameter $R=0.4$, and the estimate of the relative JES uncertainty is comparable to that for anti- $k_{t}$ jets with $R=0.6$, albeit slightly smaller because of the reduced effect of the dead material variation and the change in topocluster thresholds due to the smaller jet radius. The JES uncertainty for anti- $k_{t}$ 


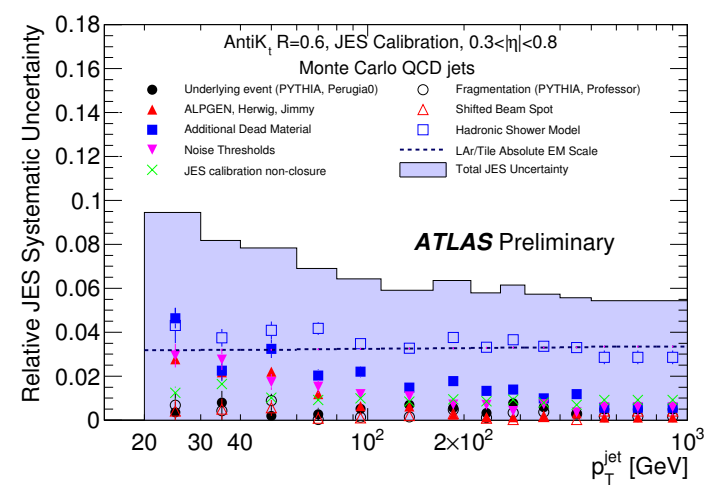

(a)

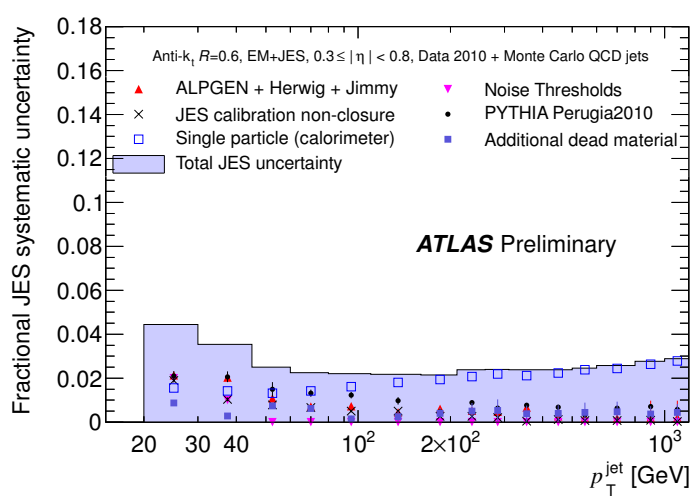

(b)

Figure D.8.:Relative jet energy scale systematic uncertainty as a function of $p_{\mathrm{T}}^{\text {jet }}$ for jets in the pseudorapidity region $0.3<|\eta|<0.8$ in the calorimeter barrel. The total uncertainty is shown as the solid light blue area. The individual sources are also shown, with errors from the fitting procedure if applicable. Figure (a) shows the first estimate of the JES uncertainty described in this Appendix, while Figure (b) shows the improved estimate used in the analysis of the full 2010 data-set.

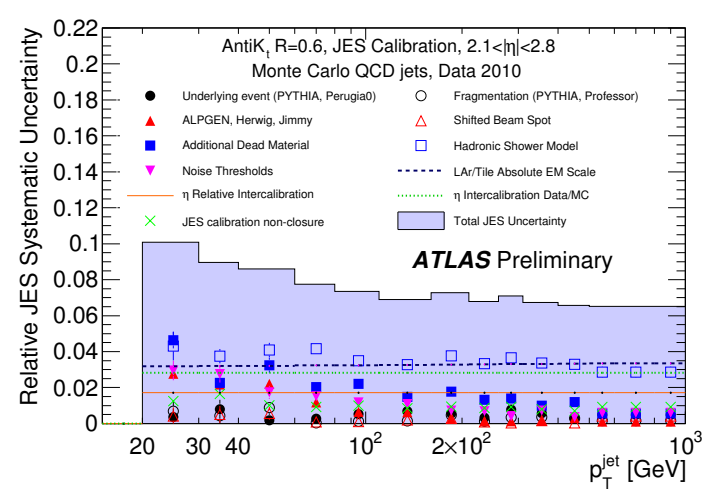

(a)

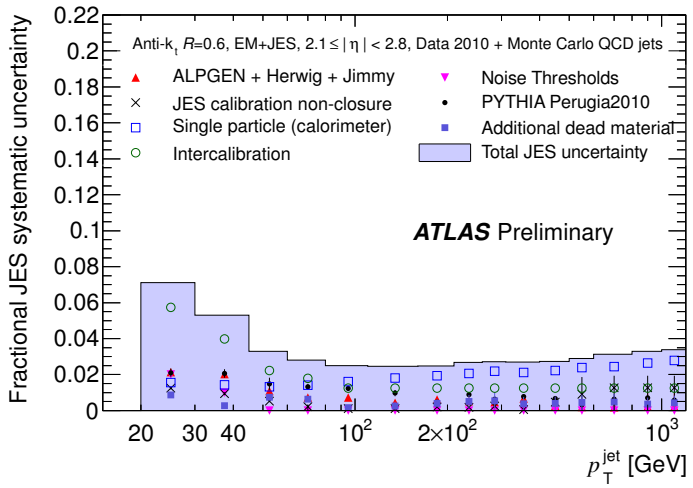

(b)

Figure D.9.:Relative jet energy scale systematic uncertainty as a function of $p_{\mathrm{T}}^{\text {jet }}$ for jets in the pseudorapidity region $2.1<|\eta|<2.8$. The JES uncertainty for the endcap is extrapolated from the barrel uncertainty, with the contribution from the $\eta$ intercalibration between central and endcap jets in data and Monte Carlo added in quadrature. The total uncertainty is shown as the solid light blue area. The individual sources are also shown, with statistical errors if applicable. Figure (a) shows the first estimate of the JES uncertainty described in this Appendix, while Figure (b) shows the improved estimate used in the analysis of the full 2010 data-set.

jets with $R=0.4$ is between $\approx 8 \%(9 \%)$ at low jet $p_{\mathrm{T}}^{\text {jet }}$ and $\approx 6 \%(7 \%)$ for jets with $p_{T}>60$ $\mathrm{GeV}$ in the central (endcap) region, and it is summarized in Table D.2 . 
Table D.1.:Summary of maximum jet energy scale systematic uncertainties for different jet $p_{\mathrm{T}}^{\mathrm{jet}}$ and $\eta$ regions from Monte Carlo-based study for anti- $k_{t}$ jets with $R=0.6$

\begin{tabular}{|c|c|c|}
\hline $\boldsymbol{\eta}$ region & \multicolumn{2}{|c|}{ Maximum relative JES Uncertainty } \\
\hline & $\boldsymbol{p}_{\boldsymbol{T}}^{\text {jet }}>\mathbf{2 0} \mathbf{G e V}$ & $\boldsymbol{p}_{\boldsymbol{T}}^{\text {jet }}>\mathbf{6 0} \mathbf{G e V}$ \\
\hline $0<|\eta|<0.3$ & $9.4 \%$ & $6.9 \%$ \\
$0.3<|\eta|<0.8$ & $9.4 \%$ & $6.8 \%$ \\
$0.8<|\eta|<1.2$ & $9.3 \%$ & $7.0 \%$ \\
$1.2<|\eta|<2.1$ & $9.5 \%$ & $6.9 \%$ \\
$2.1<|\eta|<2.8$ & $10 \%$ & $7.6 \%$ \\
\hline
\end{tabular}

Table D.2.:Summary of maximum jet energy scale systematic uncertainties for different jet $p_{\mathrm{T}}^{\mathrm{jet}}$ and $\eta$ regions from Monte Carlo-based study for anti- $k_{t}$ jets with $R=0.4$

\begin{tabular}{|c|c|c|}
\hline $\boldsymbol{\eta}$ region & \multicolumn{2}{|c|}{ Maximum relative JES Uncertainty } \\
\hline & $\boldsymbol{p}_{\boldsymbol{T}}^{\text {jet }}>\mathbf{2 0} \mathbf{G e V}$ & $\boldsymbol{p}_{\boldsymbol{T}}^{\text {jet }}>\mathbf{6 0} \mathbf{G e V}$ \\
\hline $0<|\eta|<0.3$ & $7.5 \%$ & $5.8 \%$ \\
$0.3<|\eta|<0.8$ & $7.9 \%$ & $5.9 \%$ \\
$0.8<|\eta|<1.2$ & $7.4 \%$ & $5.9 \%$ \\
$1.2<|\eta|<2.1$ & $8.0 \%$ & $6.0 \%$ \\
$2.1<|\eta|<2.8$ & $8.8 \%$ & $6.8 \%$ \\
\hline
\end{tabular}




\section{Appendix E.}

\section{Tables for the inclusive jet cross section measurement}

This appendix contains the tables containing the data, the theoretical prediction and the fractional theoretical and experimental uncertainties (with precision up to 1\%) for the inclusive jet cross section measurement. Only three rapidity regions $(0.3<y<0.8,2.1<y<2.8,3.6$ $<y<4.4$ ) are reported as an example, for both distance parameters of $\mathrm{R}=0.4$ and $\mathrm{R}=0.6$. All results for the other rapidity regions will be found on the HEPData website [156]. 


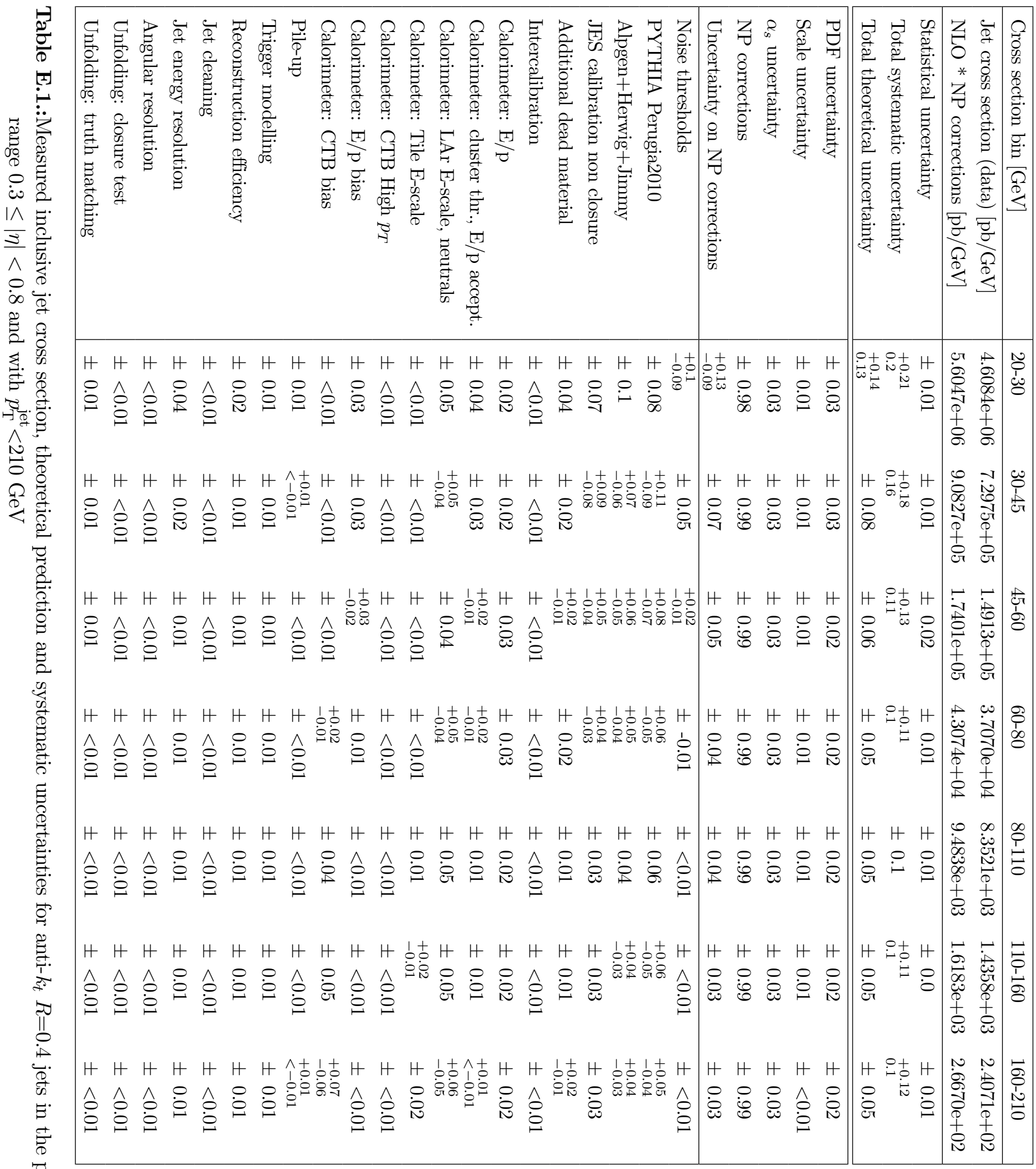




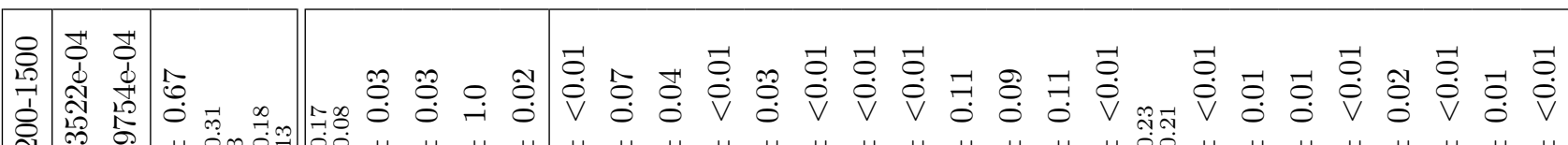
尽

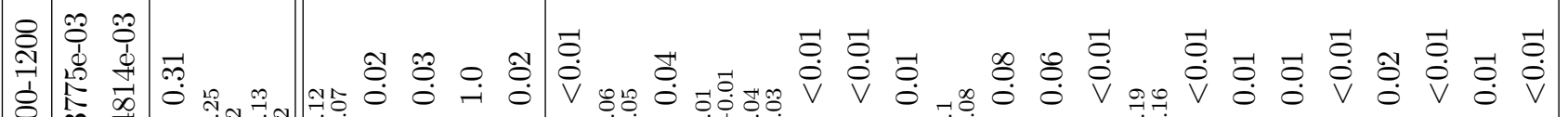

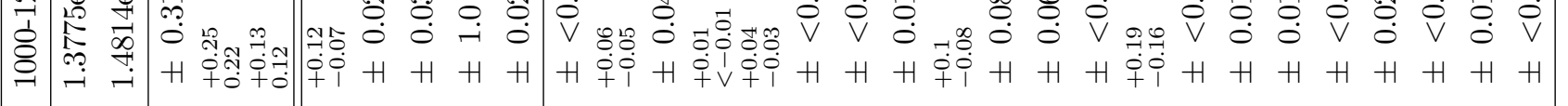

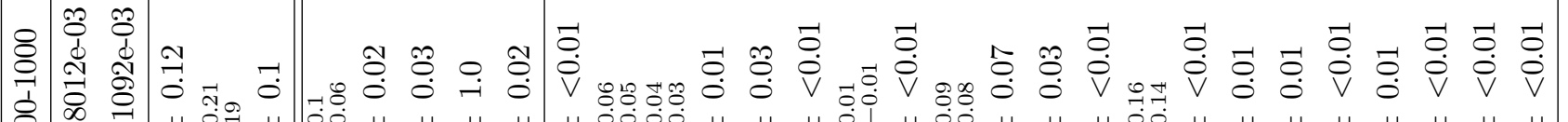

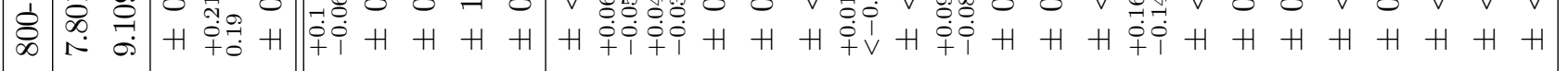

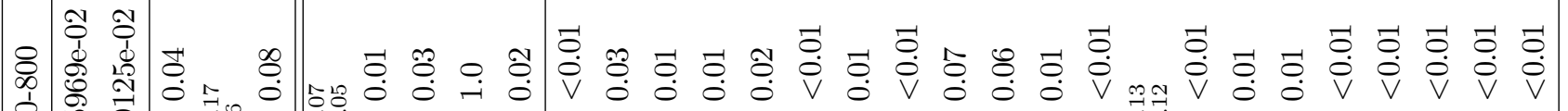

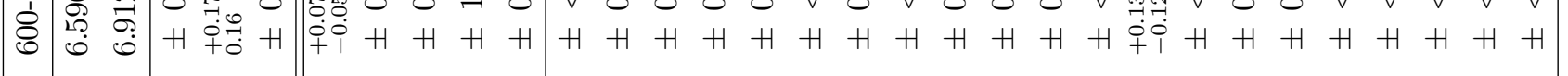

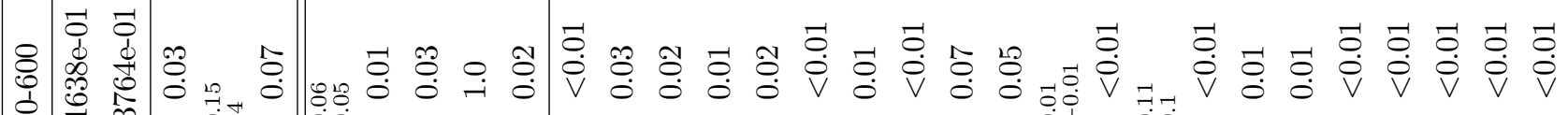

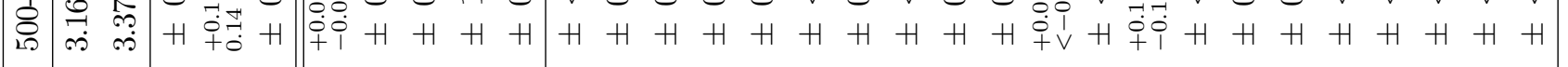

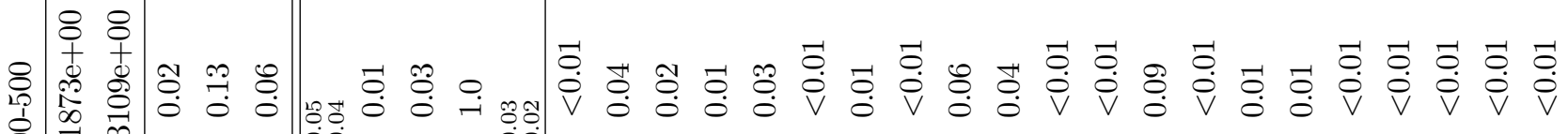

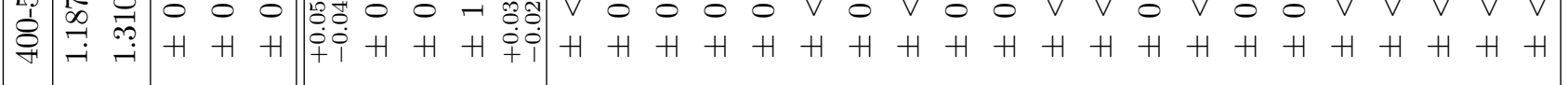

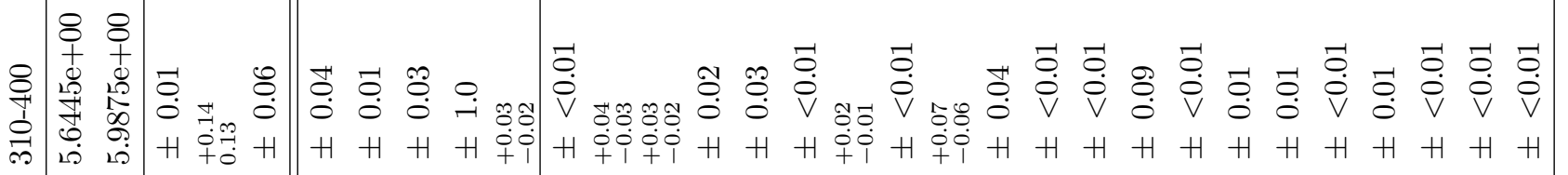

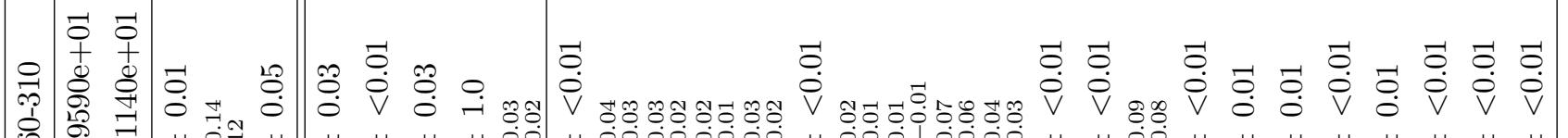

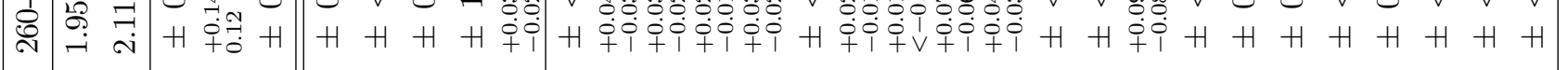

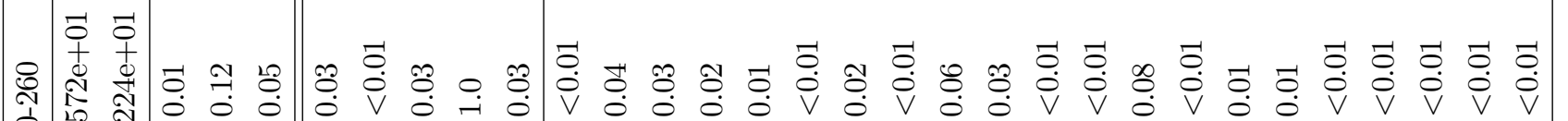

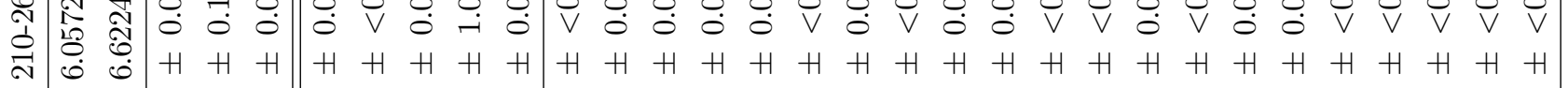

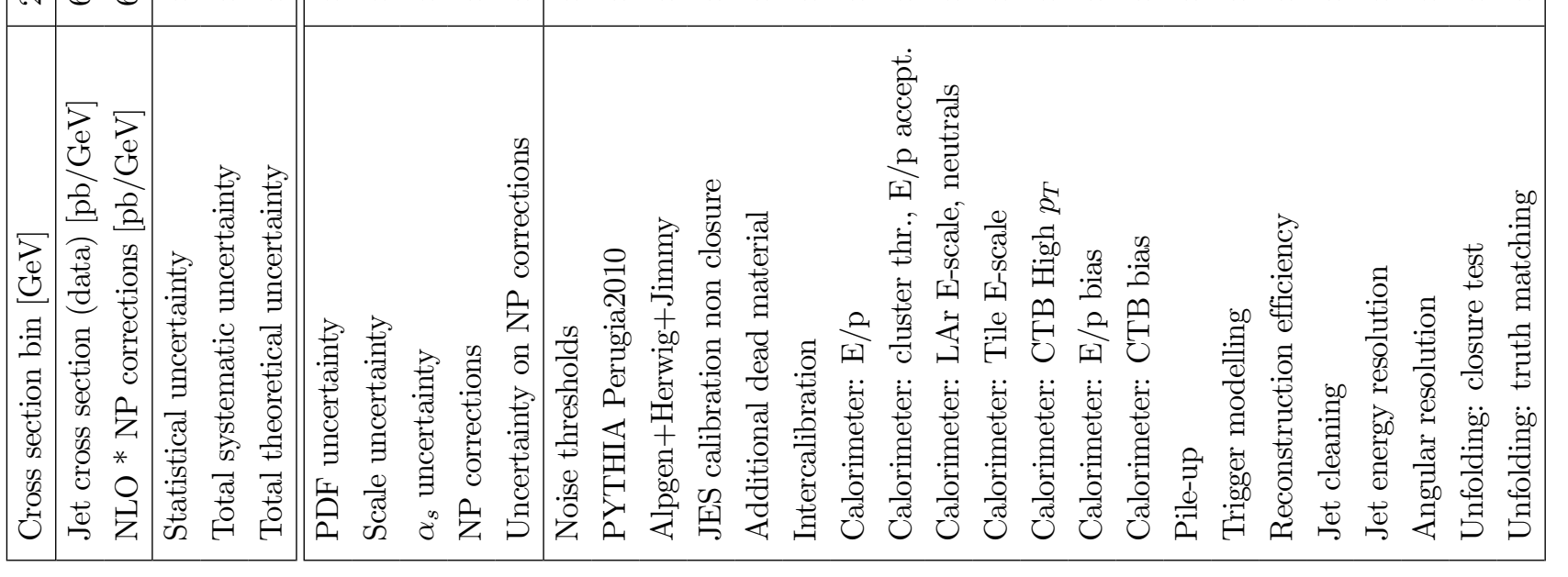

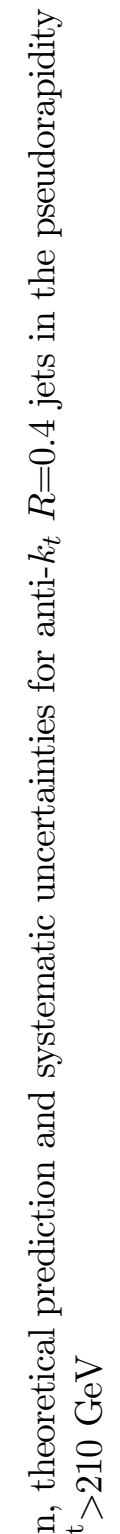

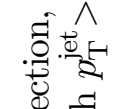
尊 월 $\stackrel{+\infty}{.} \infty$

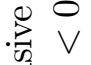
$\stackrel{3}{5} \equiv$ $\exists \overline{\mathrm{VI}}$ ?ִ $\sum_{\substack{0 \\ 0}}^{\infty}$ 


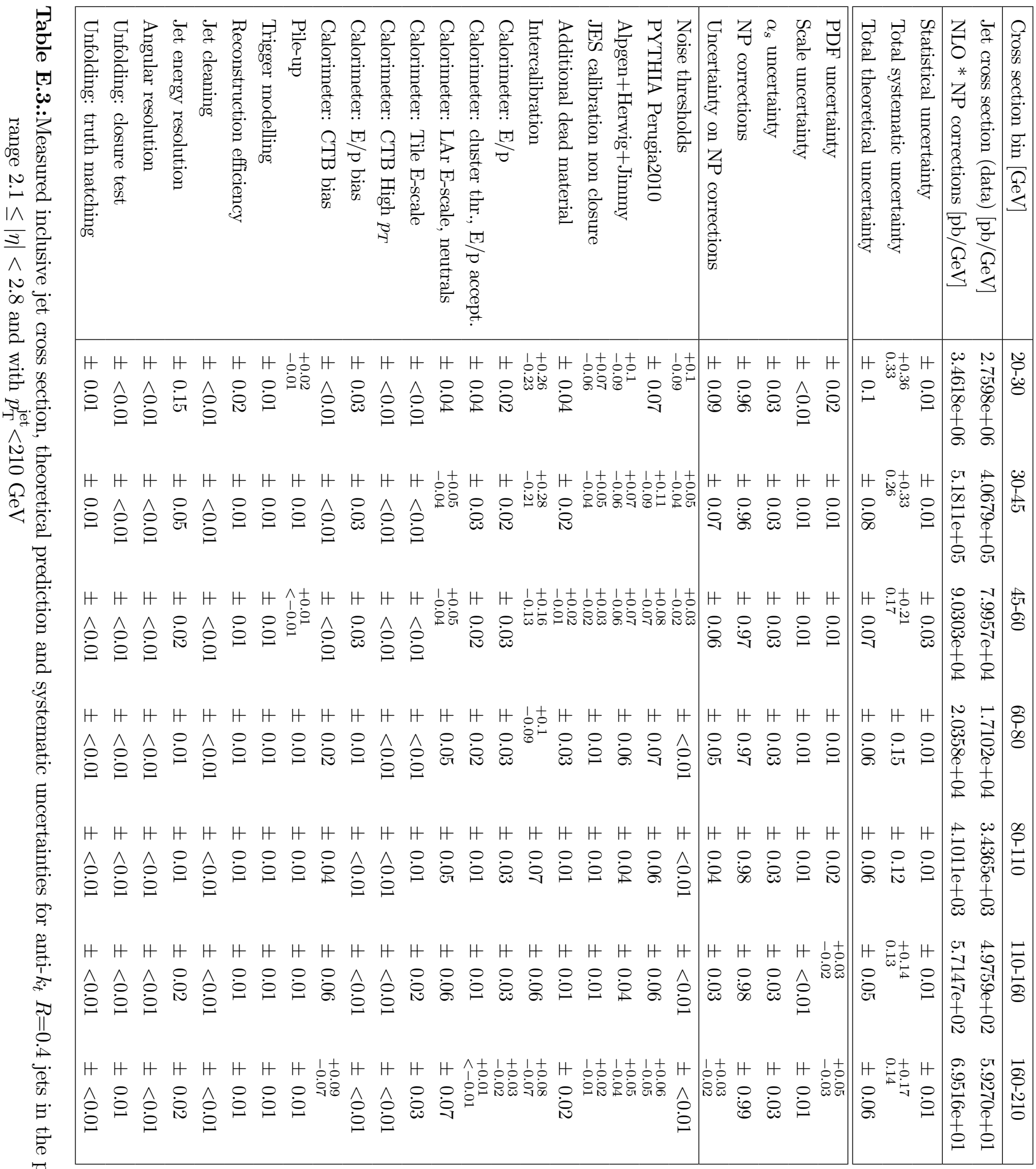




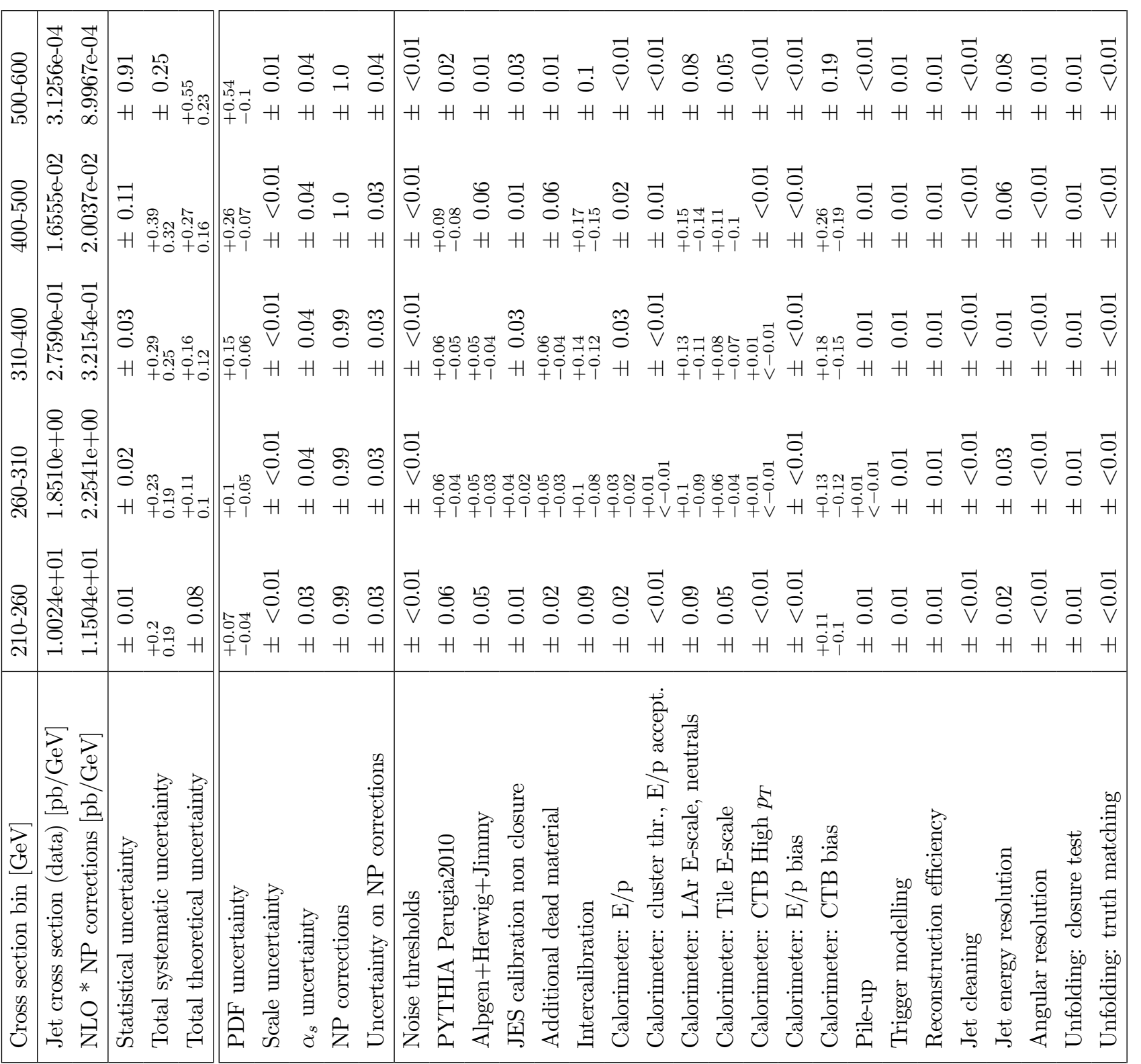




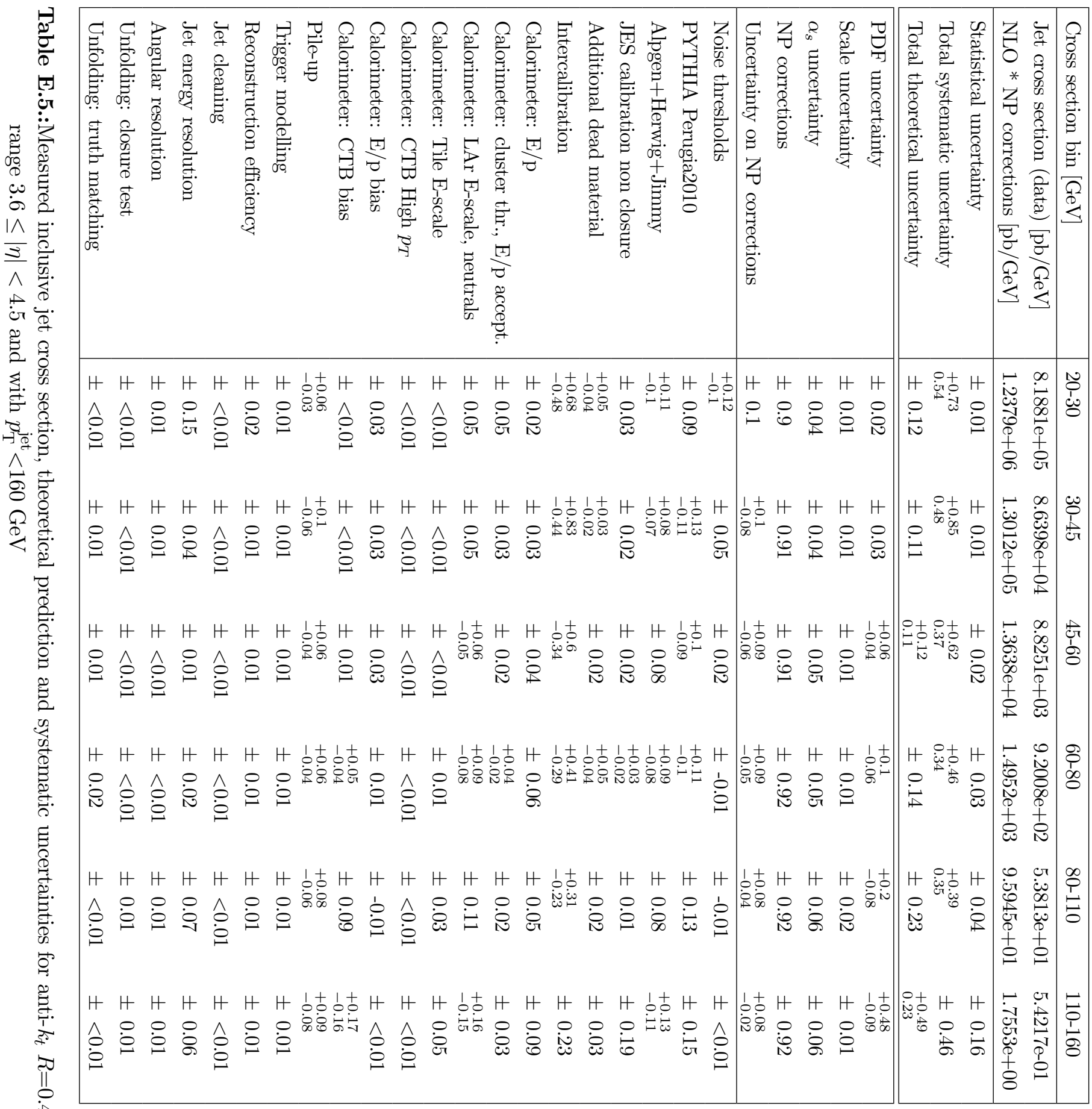




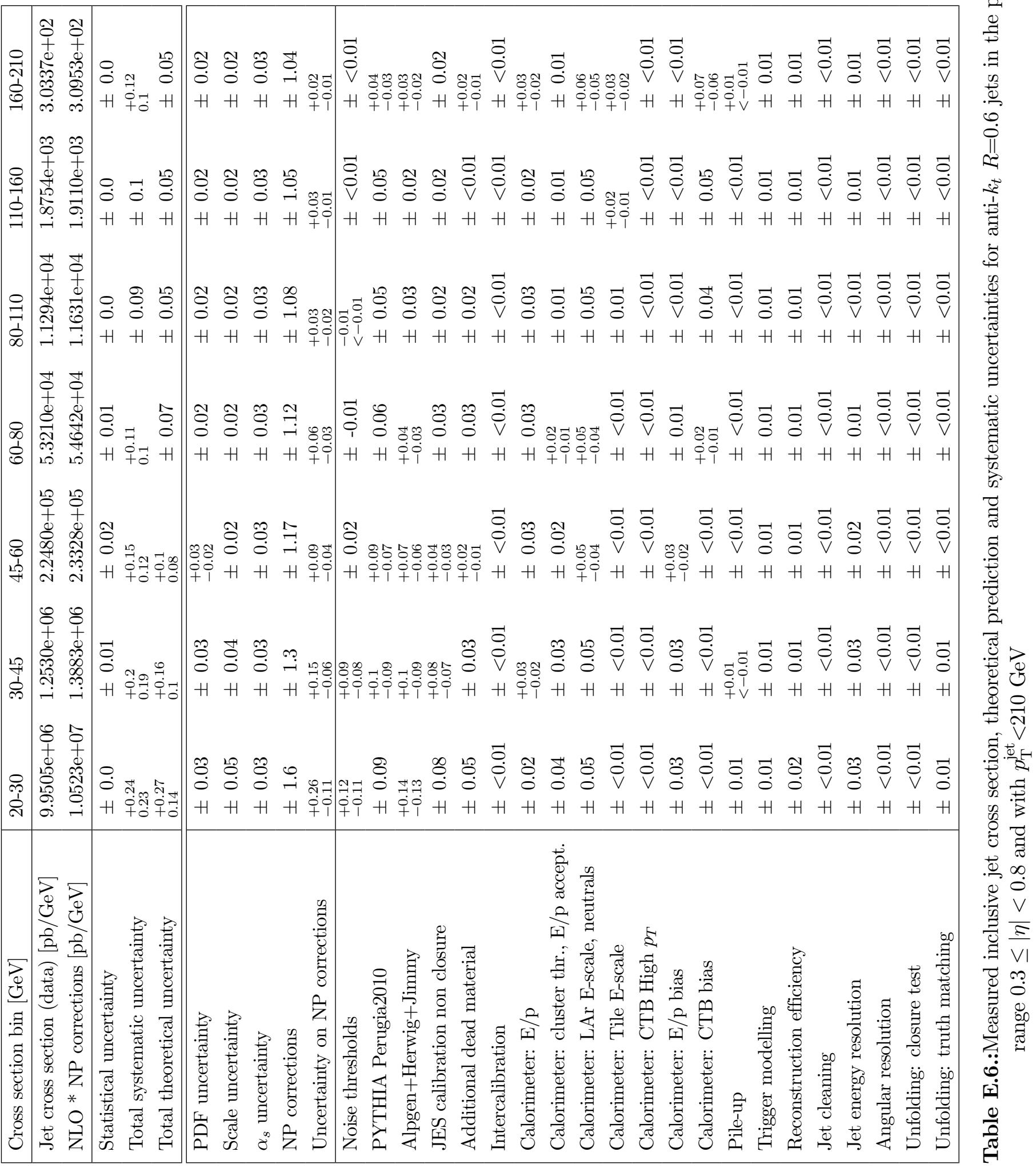




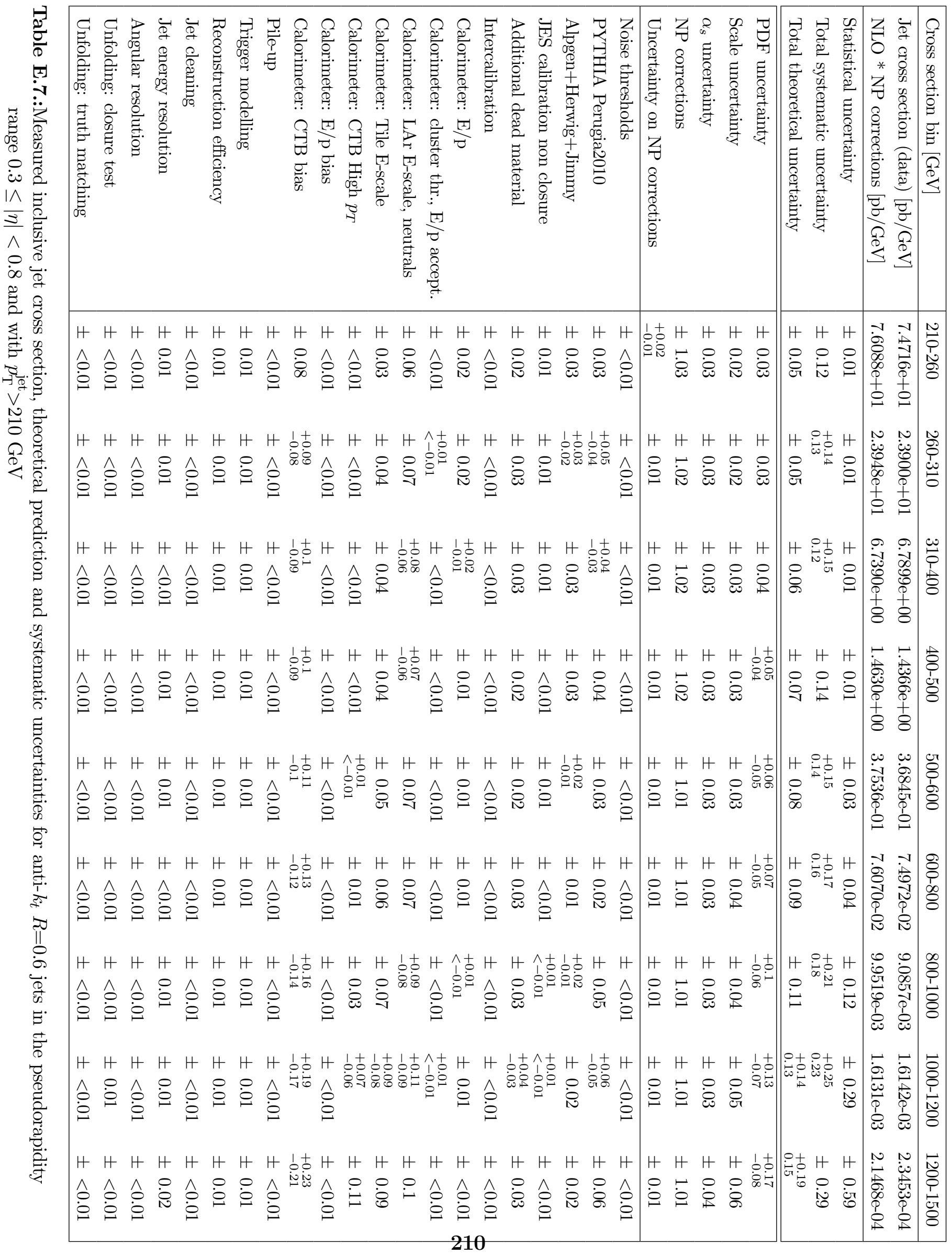




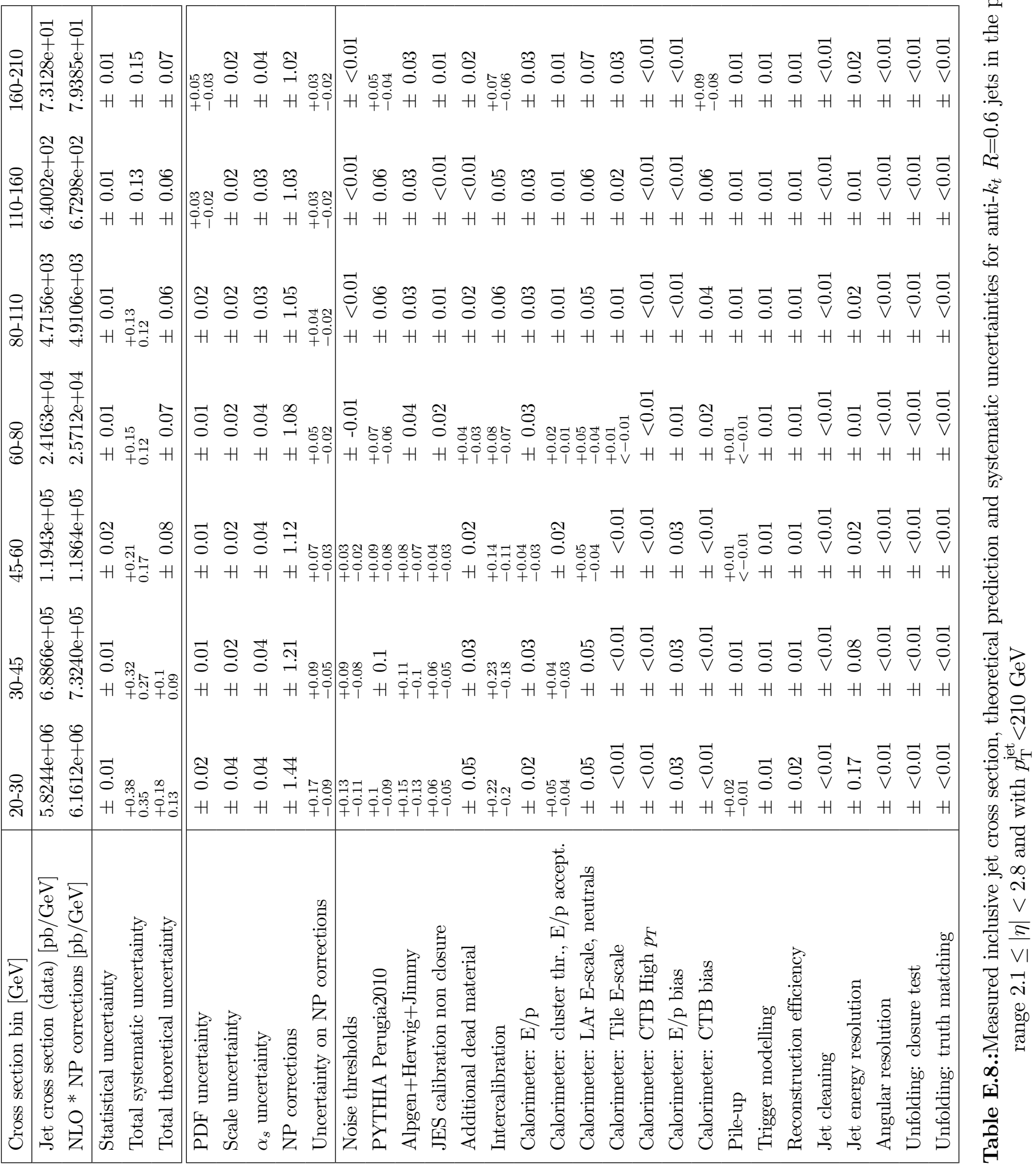




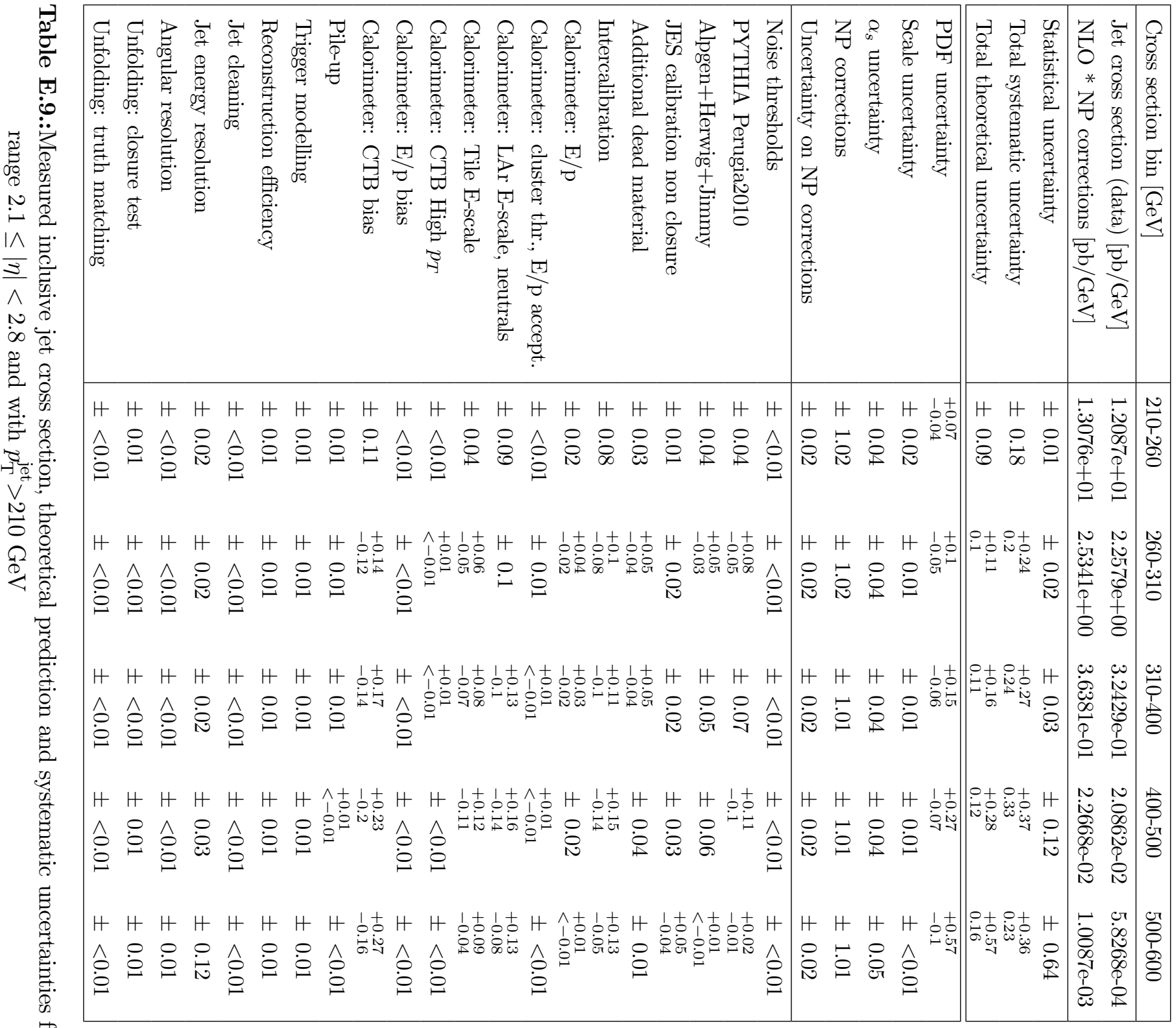

क्ष

究

II

过.

E.

索

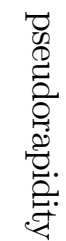




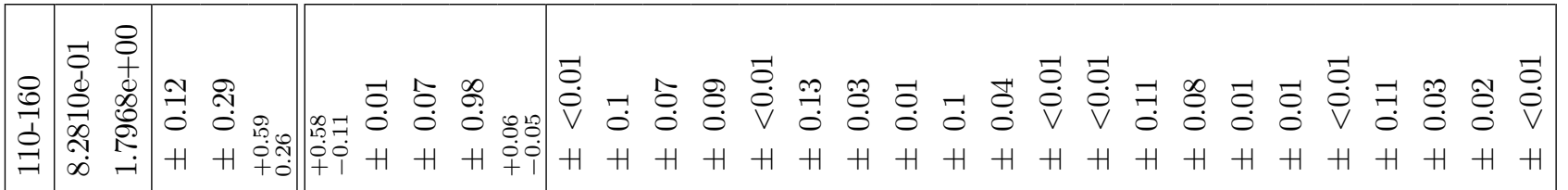

:0
0
0
0
0
0
0
0
0
0

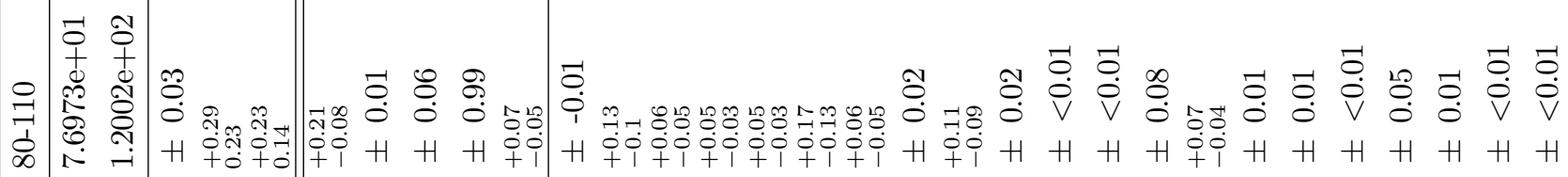

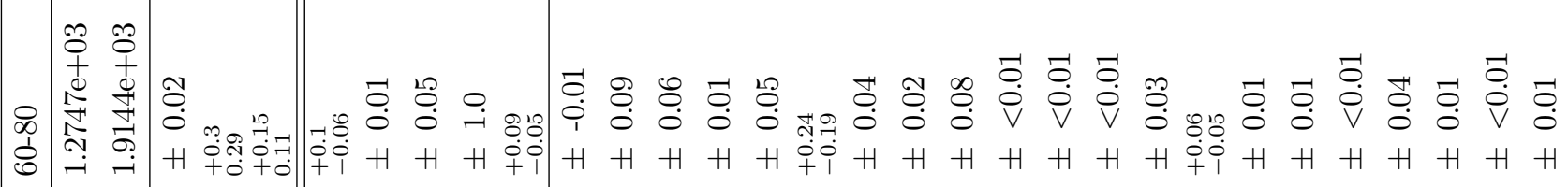

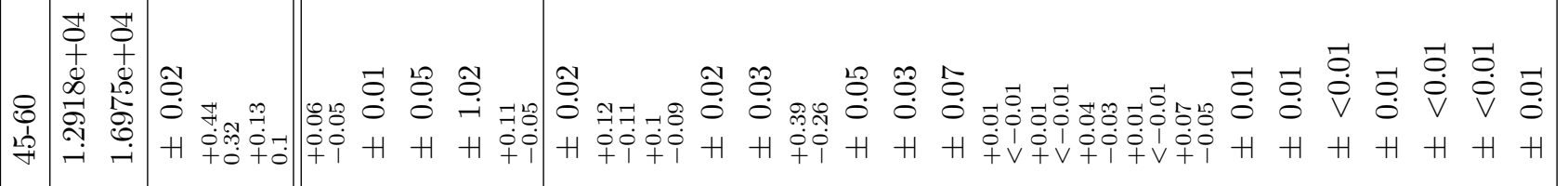

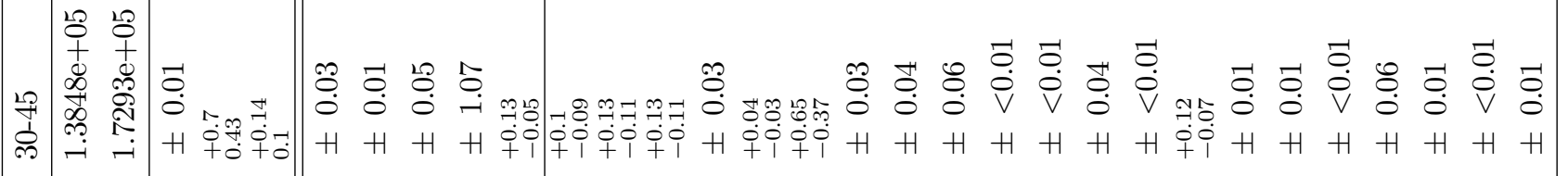

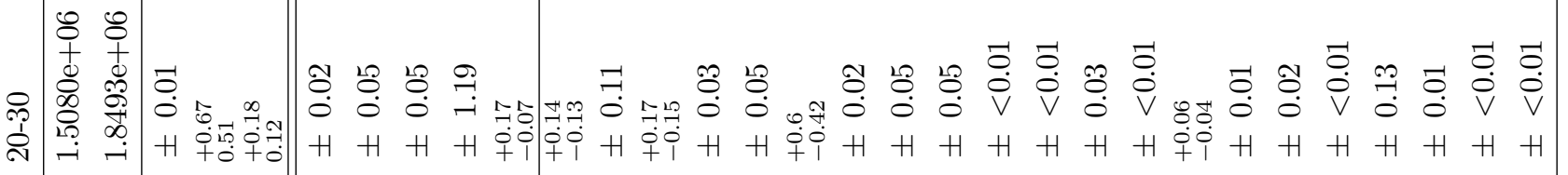

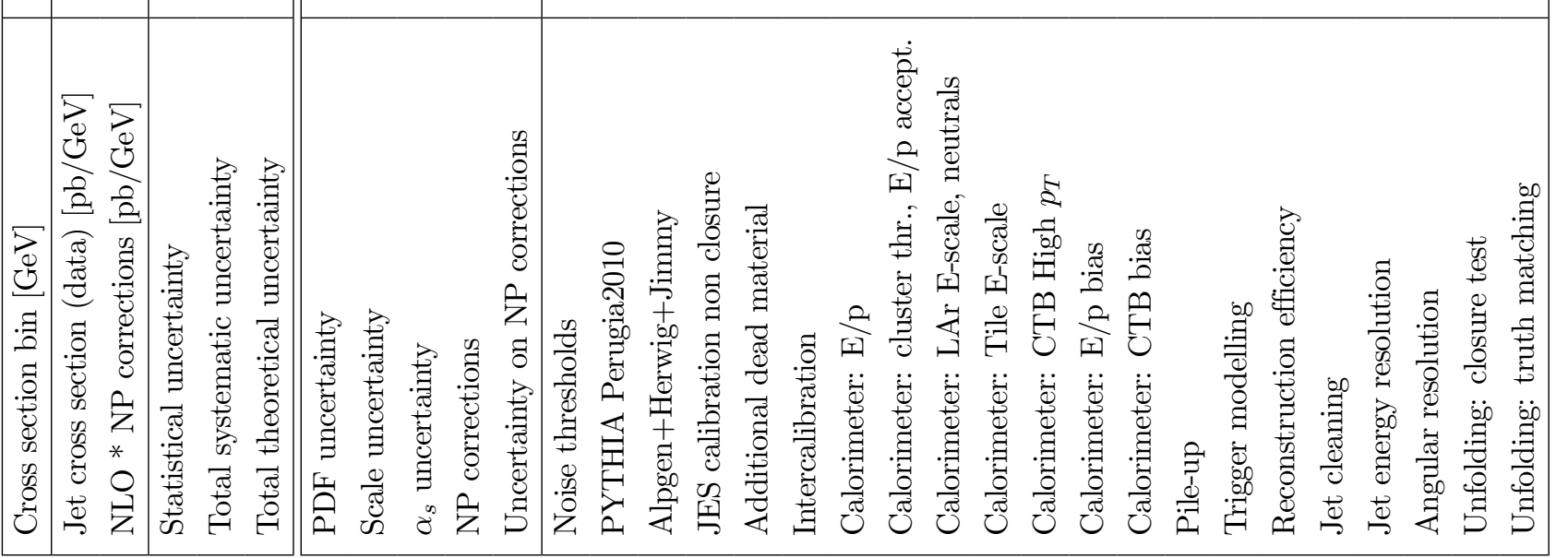

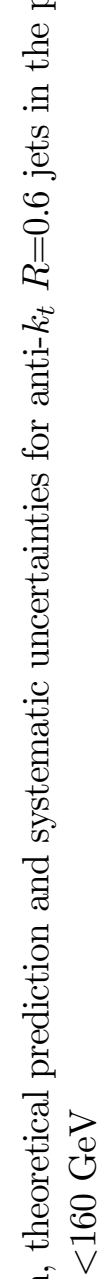

. :

总.

कृ

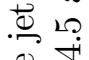

.

$\stackrel{3}{3}_{0} \equiv$

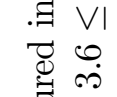

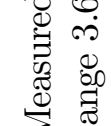

